\title{
Structure, Stability and Electronic Properties of One-Dimensional Tetrathia- and Tetraselena[8]circulene-based Materials: A Comparative DFT Study
}

Nataliya N. Karaush-Karmazin *a, Artem V. Kuklin*,b, Gleb V. Baryshnikov ${ }^{a, c}$, Lyudmila V. Begunovich ${ }^{b}$, Hans Ågren ${ }^{c, d}$ and Boris F. Minaev ${ }^{a}$

${ }^{a}$ Department of Chemistry and Nanomaterials Science, Bohdan Khmelnytsky National University, 18031, Cherkasy, Ukraine

${ }^{b}$ Department of Science and Innovations, Siberian Federal University, 79

Svobodny Av., Krasnoyarsk, 660041, Russian Federation

${ }^{c}$ Divisionof Theoretical Chemistry and Biology, School of Engineering Sciences in Chemistry, Biotechnology and Health, KTH Royal Institute of Technology, 10691

Stockholm, Sweden

${ }^{d}$ College of Chemistry and Chemical Engineering, Henan University, Kaifeng, Henan 475004 P. R. China

*Authors to whom correspondence should be addressed.

\begin{abstract}
Conjugated polymers gain much attention due to the promising applications in organic electronic device technology. In this work, we theoretically study structures and electronic properties of a novel class of nanostructures, namely onedimensional tetrathia[8]circulenes (TTC) and tetraselena[8]circulenes (TSC) predicted to be promising semiconducting soft materials. It is found that all nanoribbons are thermodynamically stable and that their electronic properties depend significantly on the type of fusing between the monomers. In particular, the band gap tends to decrease while moving from the directly fused TTC/TSC ribbons to the structures coupled via a benzene-core linker and then to the ribbons fused through a four-membered ring. Therefore, both coupling type and length of oligomers allow one to manipulate the electronic and optical properties of the
\end{abstract}


studied ribbons. The band structure calculations of infinite nanoribbons reveal direct band gaps that decrease from 2.28 to $2.14 \mathrm{eV}$ for the TTC ribbons of the first and second fusion types. The TSC structures demonstrate the same trend exhibiting band gap narrowing from 2.41 (type I) up to $2.11 \mathrm{eV}$ (type II). The type III ribbons possess the lack of periodicity due to the close-lying energy minima for the possible twisting configurations of TTC and TSC moieties relative to the linking four-membered ring.

Keywords: tetrathia[8]circulene, tetraselena[8]circulene, band gap engineering, $\pi$ conjugation, band structure, nanoribbons.

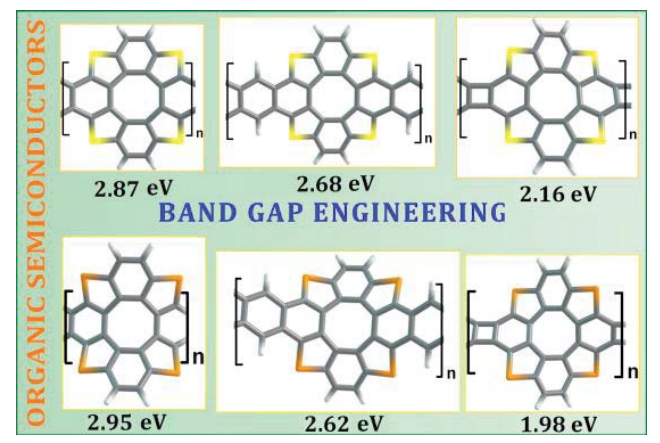

Graphical abstract 


\section{Introduction}

One- (1D) and two-dimensional (2D) conjugated polymers currently represent a rapidly developing research branch due to their potential applications in organic electronics possessing advantages over inorganic and small-molecule organic semiconductors. ${ }^{1-8}$ Modern molecular design methods make it possible to alter and engineer materials of different architectures with certain desired properties and functionality through controlled and directed assemblies of molecular building blocks in order to meet the requirements of certain organic devices. Recently, a wide range of molecules including benzene, furan, thiophene, pyridine, acetylene, etc., as well as bulky polycyclic aromatic hydrocarbons (PAHs) and polyheterocyclic structures have been successfully used as appropriate building blocks for synthetic preparation of the shape-persistent conjugated macrocycles with different dimensionality. ${ }^{1}$ The self-assembly and self-organization of porphyrins, metalloporphyrins and phthalocyanines prove the possibility to build ideal $1 \mathrm{D}$ or $2 \mathrm{D}$ extended polymers, ${ }^{2-6}$ for prospective catalytic, photovoltaic and electronic applications. Such materials can provide an efficient optical coupling leading to significantly higher optical absorption and photocatalytic activity compared to the original monomers. ${ }^{7,8}$

Recently, Osuka et al. ${ }^{9,10}$ have reported the synthesis of a free base porphyrin sheet (Fig. 1, a) consisting of four meso-meso fused porphyrin molecules as initial building units. It was shown that anomalous electronic properties including the unusual aromaticity of the porphyrin sheet arise from the central strongly antiaromatic cyclooctatetraene (COT) core which prefers to be planar due to the direct fusion of the porphyrins. Much attention has also been paid to study optoelectronic properties and the self-assembly phenomenon of phthalocyanines, which are of paramount importance for their incorporation into photovoltaic devices. ${ }^{11-13}$ In particular, phthalocyanines have a larger $\pi$-conjugated system compared to porphyrins, which leads to a radiation absorption of a longer wavelength. ${ }^{14,15}$ The high stability and insolubility of phthalocyanines in most solvents makes these compounds applicable as useful dyestuff. ${ }^{16,17}$ 
An interesting alternative class of polyheteroaromatic systems contained antiaromatic COT-cores are heterocirculenes. ${ }^{18-21}$ The electronic spectral and photophysical properties of these compounds are in the spotlight of current studies ${ }^{22-27}$ due to their promising application in organic light-emitting diodes (OLEDs) ${ }^{28-30}$ and organic field-effect transistors (OFETs) ${ }^{31-33}$ technologies. Similar to porphyrins and phthalocyanines, the inherent functionalities stored within the heterocirculene systems make them attractive building blocks for novel tailor-made $\pi$-conjugated polymers. Recently, tetraoxa[8]circulene (TOC) was predicted as an initial building unit in formation of the stable 1D and 2D TOCbased materials (Fig. 1, b-e) ${ }^{34-37}$ which represent biomimetic-type nanopores in the context of their ability to capture s- and d-block metal ions. ${ }^{38,}{ }^{39}$ In contrast to the parent tetraoxa[8]circulene molecule, TOC-polymers show strong absorption in the visible region due to the consistent growth of $\pi$-conjugation. ${ }^{34,35}$ Moreover, the electronic properties of these polymers depend significantly on the type of fusing between monomers. In particular, among TOC-polymers with three different fusion principles discussed in Ref. 37 only TOC-polymers with directly linked TOC-molecules and TOC-polymers with benzene-core linkers demonstrate semiconducting properties with 1.37 and $1.84 \mathrm{eV}$ direct band gaps, while another one in which TOC moieties linked through the four-membered ring was found to be a semimetal with $\sim 87 \mathrm{meV}$ band gap induced by spin-orbit coupling. ${ }^{37}$ 


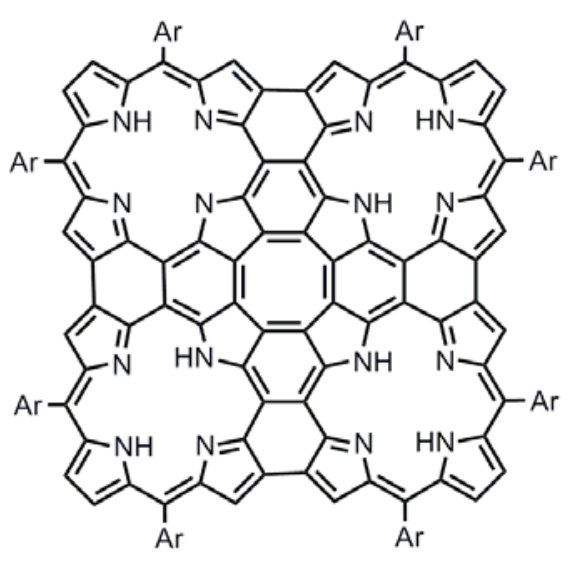

a
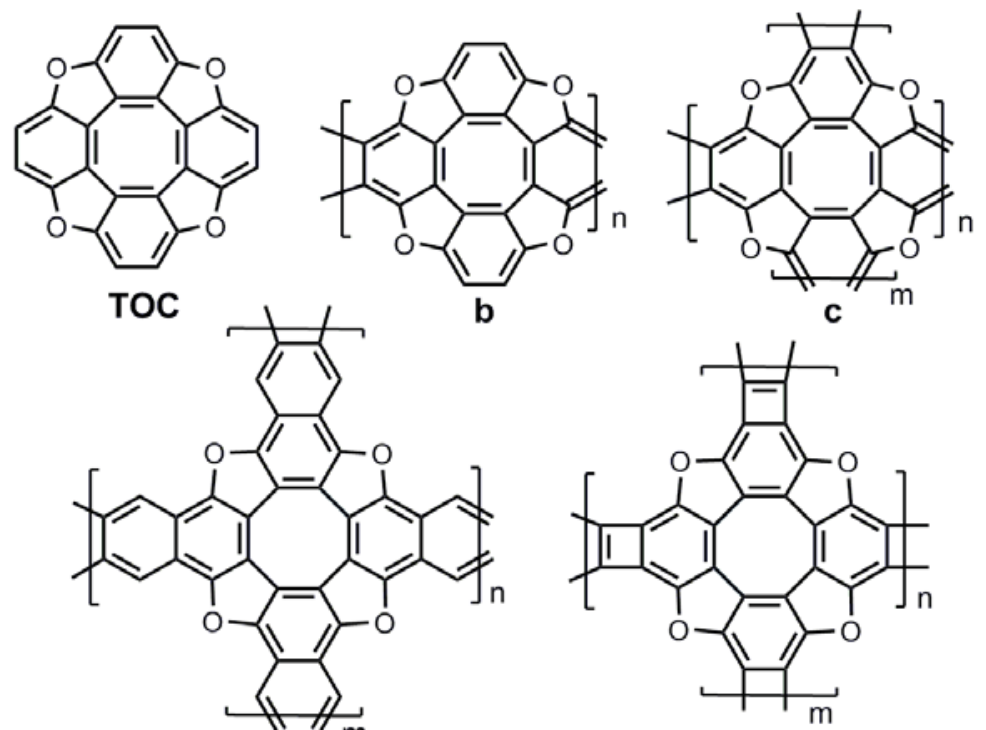

d

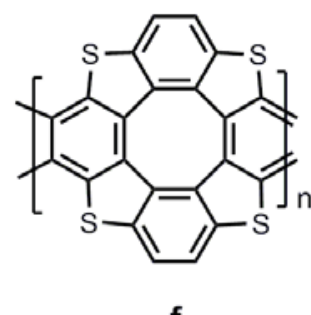

$f$

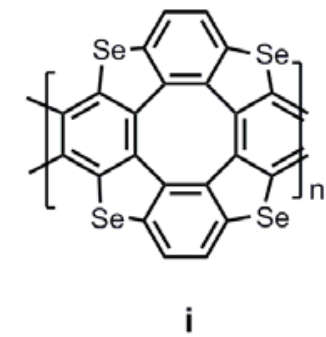

i g
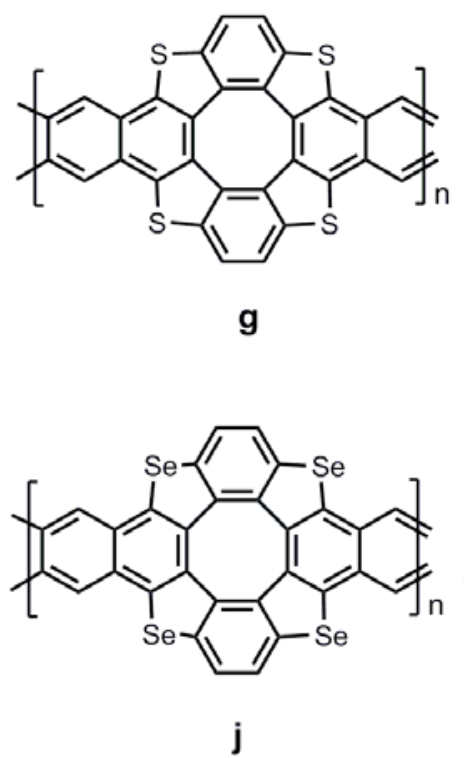

j

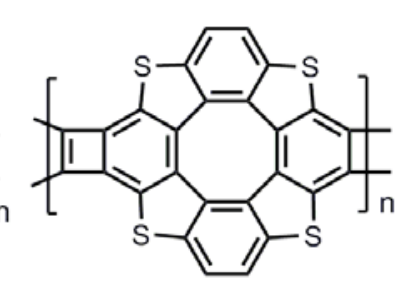

h

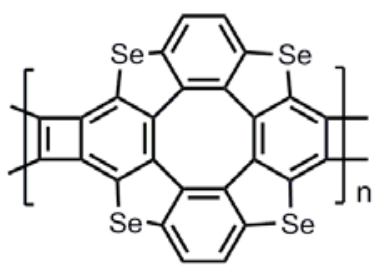

k

Fig. 1 Some examples of the 1D and 2D polymers containing porphyrin (a), $,{ }^{9}, 10$ tetraoxa[8]circulene (b-e) ${ }^{34-37}$ tetrathia[8]circulene $(\mathbf{f}-\mathbf{h})$ and tetraselena[8]circulene (i-k) monomers studied in this paper.

The methoxy-substituted TTC and TSC hetero[8]circulenes (Fig. 1) have been prepared in $2015^{40}$ and their electronic and spectral properties have also been interpreted in detail. ${ }^{41-43}$ In 2019 Miyake et al. reported a diversity-oriented approach for the synthetic preparation of tetrathia[8]circulenes which enables a convenient installation of various peripheral substituents. ${ }^{44}$ This is an effective way to fine tune the photophysical properties and control the solid-state packing of 
tetraheterocirculenes. Moreover, a great advantage of the tetrahetero[8]circulenes being effective for their functionalization is the presence of $\mathrm{CH}$ bonds which serve as the main functionalization centers in chemical transformations and possible formation of $\mathrm{n}$-dimensional materials.

In this paper, we perform a comprehensive study of two new families of 1D covalent organic polymers based on tetrathia[8]circulene (TTC) and tetraselena[8]circulene (TSC) as initial building units with different types of intermonomer fusion (Fig. 1, f-k). Depending on the fusion type between tetrahetero[8]circulene (TTC and TSC) molecules, various materials can be obtained. Such inter-monomer coupling can proceed through intermolecular dehydrohalogenation or the Ullman-type polymerization reactions which have been shown to be thermodynamically allowed. ${ }^{35}$ The ability to control intermonomer fusion significantly expands the design possibilities of novel polymers with targeted functional properties.

\section{Computational details}

The structure, UV-visible absorption spectra and band structure origin were investigated for the two series of designed 1D oligomers based on TTC and TSC building units. Both these species were constructed by n-repeating TTC and TSC monomers ( $\mathrm{n}=2-5$, and infinite band) along the 1D direction. In accordance with this principle, we have designed various TTC and TSC-based materials with three different types of fusion between monomers including the TTC or TSC-materials with directly linked TTC/TSC units (type I), fused via a benzene-core linker (type II) or through a four-membered ring (type III).

The finite TTC and TSC-based 1D structures were optimized at the B3LYP/6-31G(d) level of density functional theory (DFT) ${ }^{45-49}$ using the Gaussian 16 software package. ${ }^{50}$ The absence of imaginary vibrational wavenumbers in the calculated IR spectra indicates that a true minimum on the hypersurface of the total energy was found. The electronic absorption spectra of all the studied molecules 
were calculated by the time-dependent (TD) DFT method ${ }^{51}$ in the vacuum approximation with the same B3LYP/6-31G(d) method. ${ }^{45-49}$

The infinite units were calculated in periodic boundary conditions (PBC) within the generalized gradient approximation (DFT GGA) as realized in the Vienna Ab initio Simulation Package (VASP). ${ }^{52,53}$ The projector augmented wave (PAW) ${ }^{54}$ method and the Perdew-Burke-Ernzerhof (PBE) ${ }^{55}$ exchange functionals were employed. To correctly describe electronic properties, the long-corrected hybrid functional proposed by Heyd, Scuseria, and Ernzerhof (HSE06) was employed. ${ }^{56}$ The cutoff energy utilized for the plane wave expansion of the wave functions was set to $400 \mathrm{eV}$. The vacuum region of $15 \AA$ was set along the $\mathrm{z}$ direction to avoid interactions between neighboring images. A set of $1 \times 40 \times 1 \mathrm{k}$ points was applied according to the Monkhorst-Pack scheme ${ }^{57}$ for electronic structure calculations in the PBE approximation, while $1 \times 6 \times 1 \mathrm{k}$-points were used utilizing the HSE06 functional. The convergence tolerances of the force and electronic minimizations were set to $10^{-5} \mathrm{eV} / \AA$ and $10^{-6} \mathrm{eV}$, respectively.

\section{Results and discussion}

\subsection{Structural features}

According to X-ray data ${ }^{40}$ the free methoxy-substituted TTC and TSC molecules represent non-planar species (the $D_{2}$ symmetry point group) which was also well reproduced by our DFT calculations. ${ }^{41}$ In the case of TTC, the deviation from planarity is very slight, about $2^{\circ}$, while TSC has a more pronounced saddleshaped structure with a bent angle of the COT core of $12^{\circ}$. Such structural perturbations have been explained by insertion of more massive sulfur or selenium heteroatoms into the outer framework of the heterocirculenes. ${ }^{42}$ Therefore, it can be assumed that these circulenes being building blocks in the TTC and TSC-based structures will adopt to the shape of the free molecules along the corresponding ribbon. Indeed, our DFT calculations predict more distorted shapes for the TSCbased structures compared to those of TTC ones due to the inherent saddle shape of the initial TSC-building unit. The optimized structures of the predicted 1D 
ribbons $(\mathrm{n}=2$ and 5) are shown in Fig. 2. Their general size parameters (length and width) are presented in Table 1.

As one can see from Fig. 2, direct fusion of the TTC monomers (the first fusion type) provides a steric hindrance in the TTC-structures; therefore, the shape of ribbon with $\mathrm{n}=2$ is twisted in such a way that the sulfur atoms of adjacent thiophene rings are located as far as possible with S--S distances equal to $3.07 \AA$. Further inclusion of the TTC building unit into the molecular skeleton $(n=3-5)$ leads to the formation of the regular wave-like ribbons with distances between adjacent ridges (wavelength) of $16.7 \AA$ (Fig. 2), herewith, the S--S distances decrease to $3.02-3.06 \AA$. It should be noted that the terminal TTC fragments are more planar than the inner ones. The deviations from planarity of the COT core are $\approx 10^{\circ}$ and $\approx 15^{\circ}$ for the terminal and inner TTC macrocycles, respectively. A continuous attaching of the same building blocks in the second dimension should lead to a porous $3 \mathrm{D}$ structure with high thermoelectric performance. ${ }^{58}$

The inclusion of a benzene-core linker into the TTC ribbons (second fusion type) eliminates the problem of steric complications between TTC building blocks. The TTC ribbons remain a wave-like structure with wavelength $21.8 \AA$ with formation strongly planar acene structural part (Fig. 2). In this case, the structure of the TTC fragments tends to be planar approaching the shape of the initial free TTC molecule. The deviation from planarity of the COT core is about $5^{\circ}$ which is significantly smaller compared to the previously discussed directly fused TTCstructures.

The structure of the TTC ribbons fused through a four-membered ring (the third type) adopts linear topology in which alternating TTC macrocycles and biphenylene fragments can be distinguished. Moreover, the biphenylene fragments are strictly planar while the TTC fragments adopt a slightly corrugated structure with deviation from planarity $\approx 5^{\circ}$. The S--S distances between adjacent thiophene rings are $\sim 3.96 \AA$ that is larger compared to that for directly fused TTC-structures. 


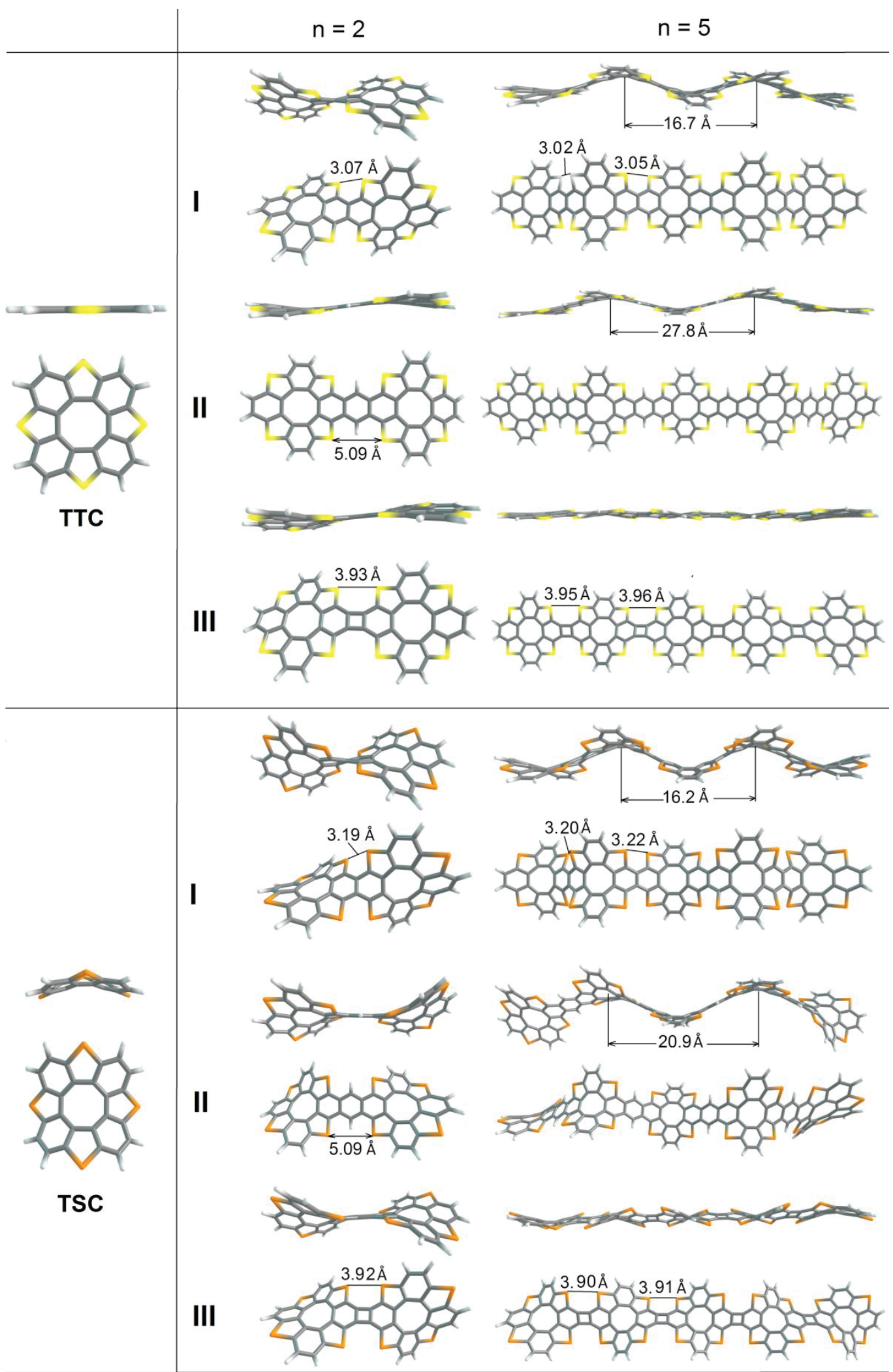

Fig. 2 Molecular representations of the TTC and TSC structures with $n=2$ and $\mathrm{n}=5$ (side and top view).

Similar to TTC-structures, the directly fused TSC-based ribbons possess a specific wave-like topology with distances between adjacent ridges of $\sim 16.2 \AA$. It 
should be noted that the direct fusion of the TSC monomers provides a more pronounced twist-type distortion of the TSC building units compared to the free TSC molecule and appearance of the saddle-shaped conformation with deviation from planarity of the COT core $\approx 25^{\circ}$. The distances between selenium atoms of adjacent selenophene rings reach up to $3.22 \AA$ along the TSC ribbons.

Table 1 The general size parameters of the TTC- and TSC-based ribbons designed by the B3LYP/6-31G(d) computations

\begin{tabular}{|c|c|c|c|c|c|c|c|}
\hline $\begin{array}{l}\text { TTC-based } \\
\text { compounds }\end{array}$ & $\mathrm{a}, \mathrm{nm}^{*}$ & $\mathrm{~b}, \mathrm{~nm}^{* *}$ & $\begin{array}{l}\text { Empirical } \\
\text { formula }\end{array}$ & $\begin{array}{l}\text { TSC-based } \\
\text { compounds }\end{array}$ & $\mathrm{a}, \mathrm{nm}^{*}$ & $\mathrm{~b}, \mathrm{~nm}^{* *}$ & $\begin{array}{l}\text { Empirical } \\
\text { formula }\end{array}$ \\
\hline \multicolumn{4}{|c|}{ Type I } & \multicolumn{4}{|c|}{ Type I } \\
\hline$n=2$ & 1.04 & 1.90 & $\mathrm{C}_{46} \mathrm{H}_{12} \mathrm{~S}_{8}$ & $\mathrm{n}=2$ & 1.04 & 1.90 & $\mathrm{C}_{46} \mathrm{H}_{12} \mathrm{Se}_{8}$ \\
\hline $\mathrm{n}=3$ & 1.02 & 2.71 & $\mathrm{C}_{68} \mathrm{H}_{16} \mathrm{~S}_{12}$ & $n=3$ & 1.00 & 2.63 & $\mathrm{C}_{68} \mathrm{H}_{16} \mathrm{Se}_{12}$ \\
\hline$n=4$ & 1.02 & 3.55 & $\mathrm{C}_{90} \mathrm{H}_{20} \mathrm{~S}_{16}$ & $\mathrm{n}=4$ & 1.00 & 3.44 & $\mathrm{C}_{90} \mathrm{H}_{20} \mathrm{Se}_{16}$ \\
\hline$n=5$ & 1.02 & 4.38 & $\mathrm{C}_{112} \mathrm{H}_{24} \mathrm{~S}_{20}$ & $\mathrm{n}=5$ & 1.00 & 4.25 & $\mathrm{C}_{112} \mathrm{H}_{24} \mathrm{Se}_{20}$ \\
\hline \multicolumn{4}{|c|}{ Type II } & \multicolumn{4}{|c|}{ Type II } \\
\hline $\mathrm{n}=2$ & 1.04 & 2.14 & $\mathrm{C}_{50} \mathrm{H}_{14} \mathrm{~S}_{8}$ & $\mathrm{n}=2$ & 1.04 & 2.12 & $\mathrm{C}_{50} \mathrm{H}_{14} \mathrm{Se}_{8}$ \\
\hline$n=3$ & 1.04 & 3.22 & $\mathrm{C}_{76} \mathrm{H}_{20} \mathrm{~S}_{12}$ & $\mathrm{n}=3$ & 1.04 & 3.09 & $\mathrm{C}_{76} \mathrm{H}_{20} \mathrm{Se}_{12}$ \\
\hline$n=4$ & 1.04 & 4.25 & $\mathrm{C}_{102} \mathrm{H}_{26} \mathrm{~S}_{16}$ & $\mathrm{n}=4$ & 1.04 & 4.14 & $\mathrm{C}_{102} \mathrm{H}_{26} \mathrm{Se}_{16}$ \\
\hline $\mathrm{n}=5$ & 1.04 & 5.41 & $\mathrm{C}_{128} \mathrm{H}_{32} \mathrm{~S}_{20}$ & $n=5$ & 1.04 & 5.11 & $\mathrm{C}_{128} \mathrm{H}_{32} \mathrm{Se}_{20}$ \\
\hline \multicolumn{4}{|c|}{ Type III } & \multicolumn{4}{|c|}{ Type III } \\
\hline $\mathrm{n}=2$ & 1.04 & 2.05 & $\mathrm{C}_{48} \mathrm{H}_{12} \mathrm{~S}_{8}$ & $\mathrm{n}=2$ & 1.04 & 2.04 & $\mathrm{C}_{48} \mathrm{H}_{12} \mathrm{Se}_{8}$ \\
\hline $\mathrm{n}=3$ & 1.04 & 3.05 & $\mathrm{C}_{72} \mathrm{H}_{16} \mathrm{~S}_{12}$ & $\mathrm{n}=3$ & 1.04 & 3.02 & $\mathrm{C}_{72} \mathrm{H}_{16} \mathrm{Se}_{12}$ \\
\hline$n=4$ & 1.04 & 4.05 & $\mathrm{C}_{96} \mathrm{H}_{20} \mathrm{~S}_{16}$ & $\mathrm{n}=4$ & 1.04 & 4.01 & $\mathrm{C}_{96} \mathrm{H}_{20} \mathrm{Se}_{16}$ \\
\hline $\mathrm{n}=5$ & 1.04 & 5.05 & $\mathrm{C}_{120} \mathrm{H}_{24} \mathrm{~S}_{20}$ & $\mathrm{n}=5$ & 1.04 & 5.00 & $\mathrm{C}_{120} \mathrm{H}_{24} \mathrm{Se}_{20}$ \\
\hline TTC & 0.87 & 0.87 & $\mathrm{C}_{24} \mathrm{H}_{8} \mathrm{~S}_{4}$ & TSC & 0.85 & 0.85 & $\mathrm{C}_{24} \mathrm{H}_{8} \mathrm{Se}_{4}$ \\
\hline
\end{tabular}

${ }^{*} \mathrm{a}$ or $^{* *} \mathrm{~b}$ - width or length of the corresponding TTC- and TSC-based structures.

Insertion of the benzene-core linker into the structure of the TSC ribbons bent them in such a way that the formed aromatic acene fragments become planar. In this case, the TSC ribbons with $n=2-3$ possess a specific curved structure, which adopts a regular wave-like topology with wavelength of $20.9 \AA$ (Fig. 2). As for TTC, the third fusion type through four-membered rings adopts linear topology with planar biphenylene fragments and oppositely arranged saddle-shaped TSC fragments (Fig. 2). 


\subsection{Electronic absorption spectra}

The calculated electronic absorption spectra of the TTC- and TSC-based ribbons are presented in Fig. 3. The electronic structure and UV-vis spectra of the methoxy-substituted TTC and TSC molecules have been previously studied in detail both experimentally and theoretically. ${ }^{41}$ In particular, both methoxysubstituted TTC and TSC molecules show a long-wavelength absorption band in the region of 320-450 $\mathrm{nm}$. The first $\mathrm{S}_{0} \rightarrow \mathrm{S}_{1}$ electronic transition calculated at 414 and $442 \mathrm{~nm}$ for TTC and TSC, respectively, is symmetry allowed with very low intensity, providing a weak fluorescence..$^{41}$ For the unsubstituted TTC and TSC molecules, the electronic transitions into the $\mathrm{S}_{1}$ excited state of the $\mathrm{X}^{1} \mathrm{~A} \rightarrow{ }^{1} \mathrm{~A}_{2}$ symmetry are forbidden $(f=0$, see ESI, Tables $\mathrm{S} 1$ and $\mathrm{S} 2 \dagger)$ in the electric-dipole approximation but allowed as magnetic-dipole transitions with relatively large magnetic moments $\left(\mu_{\mathrm{z}}\right) \approx 3.4 \mu_{\mathrm{B}}$. The transitions into the $\mathrm{S}_{2}$ excited state are strongly symmetry forbidden in both electric-dipole and magnetic-dipole approximations (see ESI, Tables S1 and S2 $\dagger$ ). The third and fourth electronic transitions into the double degenerate ${ }^{1}$ E excited state (see ESI, Tables S1 and S2 $\dagger$ ) provide strong bands at 382 and $415 \mathrm{~nm}$ for TTC and TSC molecules, respectively.

The first $S_{0} \rightarrow S_{1}$ electronic transition is allowed for most of the TTC and TSC ribbons in the calculated absorption spectra of the designed TTC and TSC species (see ESI, Tables S1 and S2 $\uparrow$ ). In particular, for the TTC ribbons of the first fusion type $(\mathrm{n}=2-5)$ the first broad band has a high intensity and takes place in the region of 430-550 nm (Fig. 3a). This band corresponds to the electronic transitions into the $\mathrm{S}_{1}$ excited state resulting from the main contribution of the $\mathrm{HOMO} \rightarrow$ LUMO configuration (Fig. 4). The highest occupied molecular orbital (HOMO) and lowest unoccupied molecular orbital (LUMO) of the TTC compounds of the first fusion type are mainly localized over the naphthalene moieties (HOMO) and central COT core (LUMO) along the whole backbone (Fig. 4). Moreover, the first band intensity is characterized by high oscillator strength values which correlates well with the TTC ribbon length and continues to 
increase linearly with increasing conjugation plane (for the TTC with $\mathrm{n}=2 f=0.48$; with $\mathrm{n}=3 f=1.02$; with $\mathrm{n}=4 f=1.62$; with $\mathrm{n}=5 f=2.26$ see ESI, Table S1 $\dagger$ ). The second band is more intense and takes place in a near UV range of $320-400 \mathrm{~nm}$ for short oligomers with $n=2$ and $n=3$; this band tends to shift to the visible region with the growth of the ribbon size (Fig. 3a).
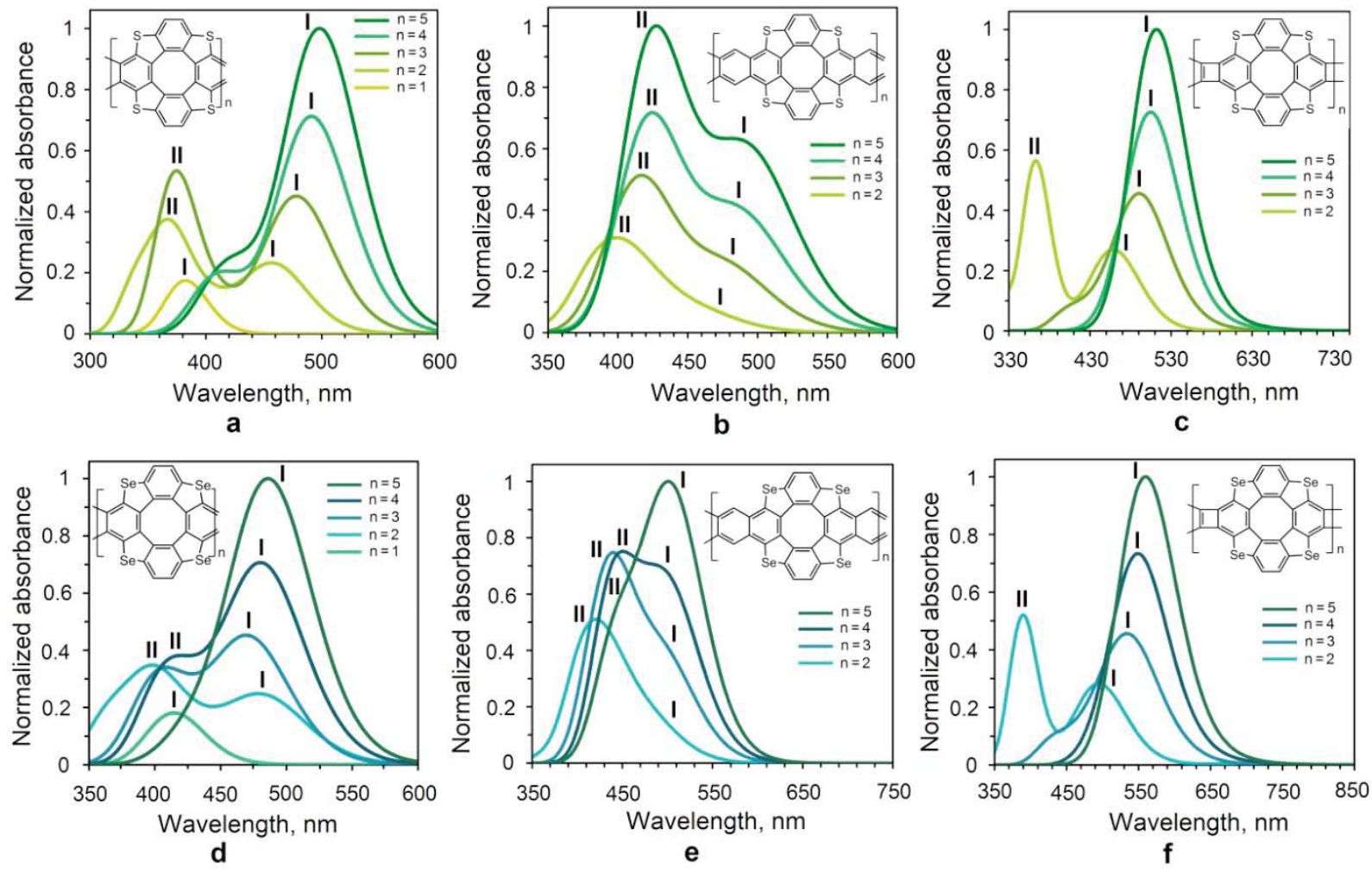

Fig. 3 Electronic absorption spectra of the TTC- and TSC-based ribbons calculated by the TD DFT/B3LYP/6-31G(d) method; I and II denote the first and second absorption bands (band half-width $3000 \mathrm{~cm}^{-1}$, Gaussian distribution function).

TTC ribbons fused via a benzene-core linker (the second fusion type) possess the first band appearing in the range $450-500 \mathrm{~nm}$ (Fig. 3b) which are clearly visible for the large ribbons ( $\mathrm{n}=4$ and $\mathrm{n}=5$ ) because the corresponding electronic transitions into $\mathrm{S}_{2}$ and/or $\mathrm{S}_{3}$ states become more intense (the oscillator strength values increase up to 3.00). The nature of these transitions can be interpreted as a mixture of the following configurations HOMO $\rightarrow$ LUMO+1, HOMO$1 \rightarrow$ LUMO, and HOMO $\rightarrow$ LUMO+2 (see ESI, Figs. S1-S12†). The first $\mathrm{S}_{0} \rightarrow \mathrm{S}_{1}$ electronic transition corresponding to the HOMO $\rightarrow$ LUMO configuration is 
allowed only for the ribbon with $n=2$, but it is forbidden for the remaining ribbons $(n=3-5)$. The second broad and much intense absorption band in the spectra of the TTC ribbons of this fusion type is predicted in the range of $380-440 \mathrm{~nm}$ which intensity proportionally increases with the growth of the ribbon length (Fig. 3b).

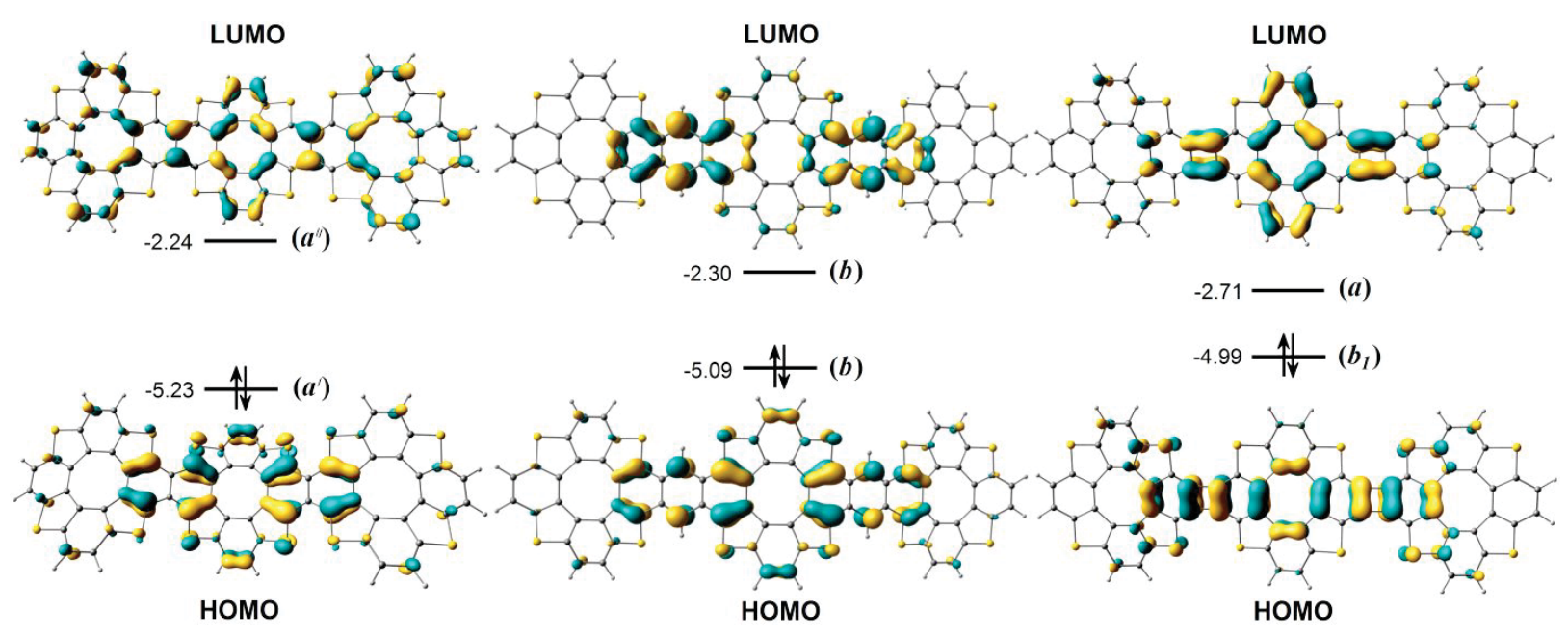

Fig. 4 Simplified molecular orbital energy diagram for the TTC ribbons of different fusion types with $n=3$. (For the TSC ribbons with $n=3$ the MOs are identical). With increasing TTC/TSC units (n) MOs have the same nature for the corresponding TTC/TSC ribbon type.

From Fig. 4 one can see that the HOMO-LUMO transition is mainly localized being close to the central part of the ribbon with some admixtures from the terminal moiety. The same trend is seen in the longer oligomers and polymers, thus showing the local exciton nature of photoconductivity and other optoelectronics properties. The $\mathrm{S}_{0} \rightarrow \mathrm{S}_{1}$ electronic transition corresponding to the HOMO $\rightarrow$ LUMO configuration was calculated in the range of $630-700 \mathrm{~nm}$ for the TTC ribbons of the third fusion type $(\mathrm{n}=2-5)$. However, it demonstrates a very weak intensity $(f=$ $0.001-0.0001)$ being practically negligible, therefore, no absorption is visible in this region of spectra (Fig. 3c). The first high-intensity band takes place in the range of 430-550 $\mathrm{nm}$ (Fig. 3c), depending on the ribbon length that is similar to the previously discussed TTC ribbons (type I). A similar trend has also been 
observed in the conjugated porphyrins. ${ }^{59}$ The second more intense absorption band takes place in the UV region for the short oligomer with $n=2$.

The absorption spectra of the designed TSC compounds represent a similar shape of bands and orbital nature of the corresponding electronic transitions, but the replacement of sulfur with selenium heteroatoms strongly affects the bands positions, i.e. the electronic transition energies (see ESI, Tables S1 and S2, $\dagger$ and Fig. 3). Similar to the TTC ribbons, the absorption spectra of the TSC compounds are also characterized by an intense size-dependent absorption in a wide visible range for the first band and in the UV-vis region for the second band. This indicates that $\pi$-conjugation in the macrocyclic system plays a key role, defining the increase of the absorption intensity with the ribbon size growth. The same phenomenon is characteristic and typical for other strongly conjugated systems such as polyenes, polyacenes and recently studied related TOC ribbons. ${ }^{59}$.

As one can see from Fig. 3d, for the TSC ribbons (type I) the first absorption band $\left(S_{0} \rightarrow S_{1}\right.$ transition) takes place in a narrow range $450-530 \mathrm{~nm}$ and reaches a maximum at $\approx 485 \mathrm{~nm}$, while for the TTC compounds of the same fusion type, the absorption maximum of the first band tends to be slightly shifted to a longer wavelength up to $\approx 498 \mathrm{~nm}$ (Fig. 3a). Similar to TTC (type I) the HOMO $\rightarrow$ LUMO configuration provided the dominant contribution to the $S_{0} \rightarrow S_{1}$ transition for TSC compounds of the same fusion type. The TSC ribbons of the second type exhibit weak absorption calculated as a shoulder in the $485-540 \mathrm{~nm}$ region (the first band) and a strong long-wave shifted absorption in the range of 380-470 nm (the second band) (Fig. 3e). In the case of the third type of TSC ribbons the first red-shifted band is calculated in the range of 460-630 nm (Fig. 3f). The $S_{0} \rightarrow S_{1}$ transition due to the HOMO $\rightarrow$ LUMO configuration is forbidden for the TSC ribbons of both II and III fusion types beginning with $n=3-5$ (Fig. 4). The detailed orbital nature interpretation of the absorption spectra for TSC compounds $n=2-5$ is presented in the ESI. $\uparrow$ 


\subsection{Band-gap engineering}

Band-gap engineering ${ }^{60,61}$ represents an attractive research area of computational materials design which allows tuning the band gap in order to achieve desired electronic and optical properties. It was shown previously that the HOMO-LUMO gap (HLG) depends on the 1D oligomer length and can be controlled by reducing or increasing the number of repeated molecular units. ${ }^{62,63}$ Moreover, the HLG value can be more precisely adjusted by the choice of the monomer coupling in the oligomeric system, i.e. the conjugation pathways. Here we deal with three different coupling types of monomers in the 1D TTC/TSC structures. Fig. 5 exhibits the HLG as a function of oligomer size $(1 / \mathrm{n})$ for all of the studied TTC and TSC-based 1D conjugated structures with $n=1-5$. As expected, the HLG diminishes with increasing number of repeated units (n) in the corresponding oligomer. This general tendency is clearly reflected in all presented series of the 1D structures with different coupling types. An interesting feature for both TTC and TSC series is that the HLG gradually decreases when we move from the ribbons with the first coupling type to the ribbons containing the conjugated anthracene system and then to the ribbons fused through fourmembered rings.

For the TTC structures of the first fusion type, the HLG values shrink very slowly up to $2.87 \mathrm{eV}$ for oligomers with $\mathrm{n}=5$. The inclusion of the benzene-core linker into the TTC structure leads to a faster decrease of the HLG up to $2.68 \mathrm{eV}$ for TTC with $n=5$. This fact can be explained by an expansion of the $\pi$ conjugation system due to the formation of the cyclic anthracene structure. The HOMO and LUMO are primarily localized over the anthracene moieties along the whole backbone (Fig. 4). For the TTCs linked through a four-membered ring, the HLG decreases up to $2.16 \mathrm{eV}$ because of the enhancement of $\pi$-conjugation in the macrocyclic system. In this case, the TTC monomers are fused with the formation of biphenylene fragments, which subsequently couple with each other through the $-\mathrm{C}-\mathrm{C}-$ bridges of the central COT core forming a strongly $\pi$-conjugated chain. 
The corresponding HOMO and LUMO are localized over the biphenylene and COT core continuously along the whole TTC backbone (Fig. 4).
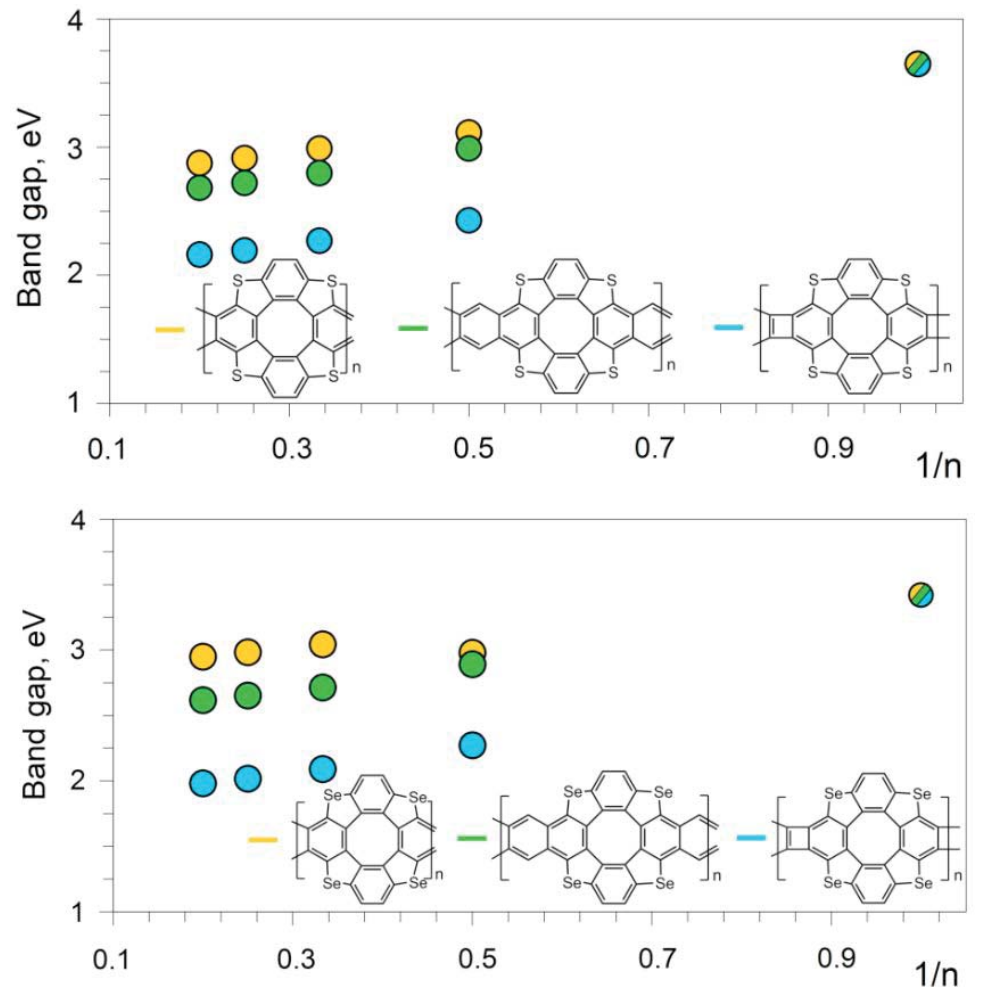

Fig. 5 The length dependence behaviour of the band gap for the TTC- and TSCbased 1D oligomers ( $\mathrm{n}$ denote the numbers of the TTC and TSC monomers). Yellow, green and blue colors correspond to I, II and III types of ribbons.

The same tendency is observed for the TSC structures with different fusion types. In particular, replacing sulfur with selenium heteroatoms leads to a slower decrease in the band gap values (up to $2.95 \mathrm{eV}$ ) for the TSCs $(\mathrm{n}=5)$ of the first fusion type compared to those for TTCs. This is due to weakening of the $\pi$ conjugation because of steric complications in TSC ribbons of this type (see Structural part). In the case of TSC of the second type, the HLG decreases up to $2.62 \mathrm{eV}$ with $\mathrm{n}=5$ similar to the corresponding TTCs. The TSC of the third type demonstrates HLG reduction up to $1.98 \mathrm{eV}$. We have neglected spin-orbit coupling ${ }^{64}$ throughout all DFT calculations. Thus, we have to mention that some heavy atom effect ${ }^{65}$ could be additionally expected in the TTC and TSC types of ribbons, while it will influence mostly the singlet-triplet transitions, which have not been considered in the present paper. 


\subsection{Structure and electronic properties of the infinite nanoribbons}

Having the repeated units with certain wavelengths from finite structures calculations, we designed periodic nanoribbons of type I and type II. The relaxed structures demonstrate the lattice parameters $a=16.51$ (16.70) and 21.10 (19.9) $\AA$ for S(Se) type I and type II nanoribbons, respectively, that is slightly different from the respective oligomers. At the same time, the general features of the geometry are preserved. The decrease in wavelength parameter for the Se type II nanoribbons results from higher Gaussian curvature of the ribbons. The type III structure was not considered in the periodic approximation due to the absence of dependence on the number of monomers. This means that increasing oligomer length from 2 up to 5 units leads to a new structure with no periodicity at every step. The lack of periodicity for type III ribbons is likely caused by the close-lying energy minima for the possible twisting configurations of TTC and TSC moieties relative to the linking four-membered ring. As a result, type III ribbons are predicted to be conformationally soft through the small inter-monomer distortions leading to the formally non-periodic infinite structures. However, it may be possible that type III ribbons demonstrate periodicity with the larger size of the unit cell.

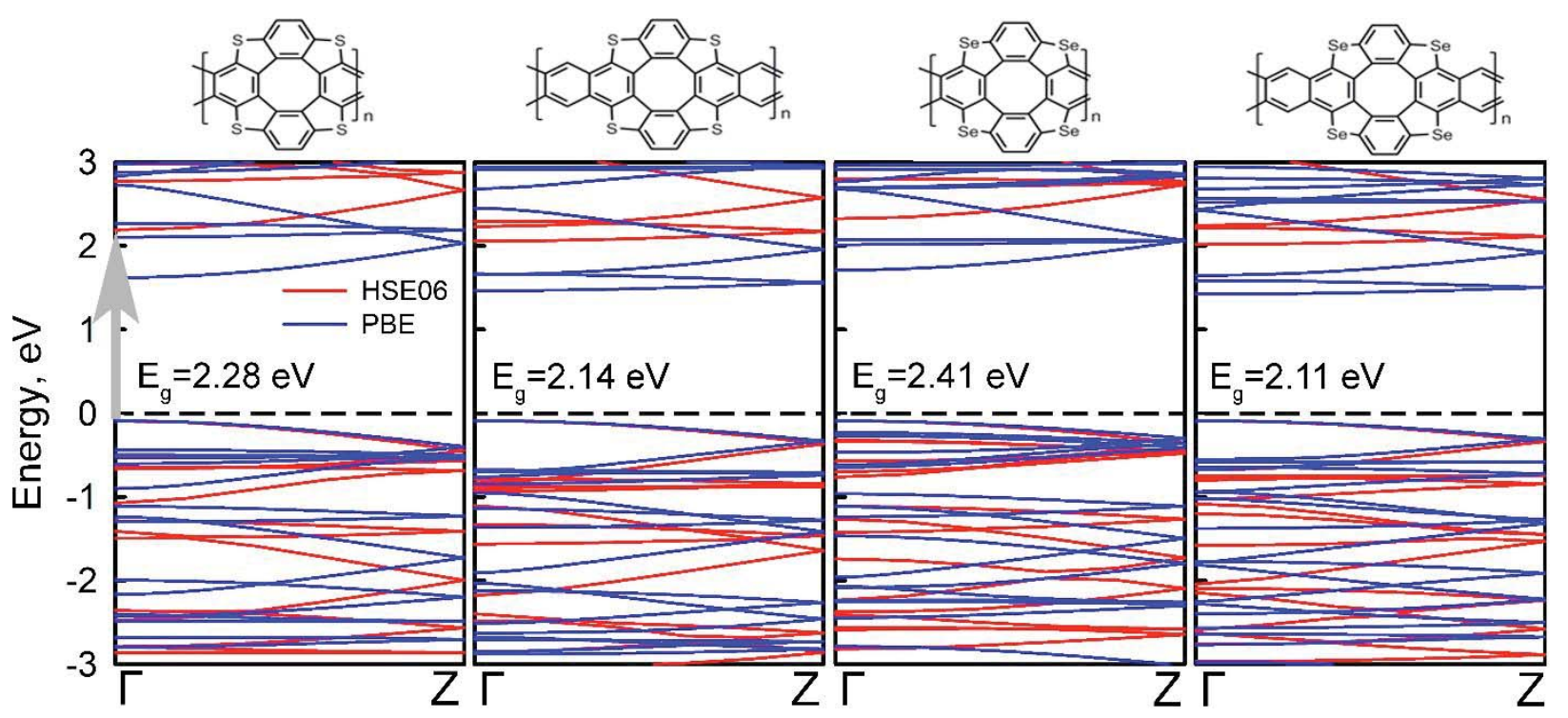

Fig. 6 Band structures of the TTC-and TSC-based 1D polymers calculated at the PBE (blue) and HSE06 (red) levels of theory. The Fermi level is set to $0 \mathrm{eV}$. 
Band structure calculations (Fig. 6) for type I and II ribbons reveal 2.11$2.41 \mathrm{eV}$ band gaps calculated at the HSE06 level of theory that depend on the fusion type and composition. These gaps are generally $\sim 0.6 \mathrm{eV}$ larger compared to those calculated within the PBE functional. While direct fusion reveals a minor increase in band gap width with replacement of $\mathrm{S}$ by Se, the second type nanoribbons demonstrate almost the same gaps for the infinite ribbons. As for finite oligomers, the inclusion of the benzene-core linker into the TTC and TSC structures leads to a faster decrease of the HLG. It is important to note that the molecular nanoribbons possess direct band gaps of a size that are promising for optoelectronic applications. It is notable that the studied nanostructures have elastic constants in the periodic direction predicted to be 10-30 GPa, something that reflects their softness and makes them possible candidates to piezo- and thermoelectric materials. ${ }^{58}$

\section{Conclusions}

In this paper, we have presented a comprehensive DFT study of the electronic structure and spectral properties for the two series of novel one-dimensional tetrathia[8]circulene and tetraselena[8]circulene-based nanostructures with different fusion types. The directly fused (type I) and fused via benzene-core linker (type II) monomers in tetrathia[8]circulene and tetraselena[8]circulene ribbons form wave-like structures while ribbons fused through a four-membered ring (type III) adopt a linear topology with slight rotation of the monomers. All the studied 1D tetrathia[8]circulene and tetraselena[8]circulene compounds demonstrate a strong size-dependent visible absorption in the electronic spectra due to specific $\pi$ conjugation in the TTC- and TSC-based systems. The materials possess variable middle-range direct band gaps, making them attractive candidates for solar cell technology and optoelectronic applications.

\section{Conflicts of interest}

There are no conflicts to declare. 


\section{Acknowledgements}

The research was carried out under the expenses of the Russian Science Foundation (Project No.19-73-10015). This work was also supported by the Ministry of Education and Science of Ukraine (projects no. 0117 U003908 and 0118U003862), the Carl Tryggers Foundation (Grant No. CTS 17:514) and the Olle Engkvist Byggmästare foundation (contract No. 189-0223). The calculations were performed with the computational resources provided by the HighPerformance Computing Center North (HPC2N) in Umeå, Sweden, through the project “Multiphysics Modeling of Molecular Materials” SNIC 2019-2-41.

\section{References}

1. X. Guo, M. Baumgarten and K. Müllen, Progress in Polymer Science, 2013, 38, 1832.

2. G. M. Mamardashvili, N. Zh. Mamardashvili and O. I. Koifman, Russ. Chem. Rev., 2008, 77, 59.

3. C. J. Medforth, Z. Wang, K. E. Martin, Y. Song, J. L. Jacobsenc and J. A. Shelnutt, Chem. Commun., 2009, 7261.

4. C. M. Drain, A. Varotto and I. Radivojevic, Chem. Rev., 2009, 109, 1630.

5. M. Abel, S.Clair, O.Ourdjini, M.Mossoyan and L. Porte, J. Am. Chem. Soc., 2011, 133, 1203.

6. L. Latos-Grazynski, in The Porphyrin Handbook, ed. K. M. Kadish, K. M. Smith and R. Guilard, Academic Press, New York, 2000, 2, 361.

7. N. Zhang, L. Wang, H. Wang, R. Cao, J. Wang, F. Bai and H. Fan, Nano Lett., 2018, 18, 1560.

8. N. Aratani, D. Kim and A. Osuka, Acc. Chem. Res., 2009, 42, 1922.

9. Y. Nakamura, N. Aratani, H. Shinokubo, A. Takagi, T. Kawai, T. Matsumoto, Z. S. Yoon, D. Y. Kim, T. K. Ahn, D. Kim, A. Muranaka, N. Kobayashi and A. Osuka, J. Am. Chem. Soc., 2006, 128, 4119. 
10. Y. Nakamura, N. Aratani, K. Furukawa and A. Osuka, Tetrahedron, 2008, 64, 11433.

11. C. G. Claessens, U. Hahn and T. Torres, Chem Rec., 2008, 8, 75.

12. M. Abel, S. Clair, O. Ourdjini, M. Mossoyan and L. Porte, J. Am. Chem. Soc., 2011, 133, 1203.

13. M. Urbani, G. de la Torre, M. K. Nazeeruddin and T. Torres, Chem. Soc. Rev., 2019, 48, 2738.

14. J. R. Darwent, I. Mc Cubbin and G. Porter, J. Chem. Soc., Farad. Trans., 1982, 2, 903.

15. J. R. Darwent, P. Douglas, A. Harriman, G. Porter and M.-C. Richoux, Coor. Chem. Rev.,1982, 44, 83.

16. G. Di Carlo, A. O. Biroli, F. Tessore, S. Caramori and M. Pizzotti, Coord. Chem. Rev., 2018, 358, 153.

17. M. Urbani, M.-E. Ragoussia, M. K. Nazeeruddin and T. Torres, Coord. Chem. Rev., 2019, 381, 1.

18. G. V. Baryshnikov, B. F. Minaev and V. A. Minaeva, Russ. Chem. Rev., 2015, 84, 455 .

19. T. Hensel, N. N. Andersen, M. Plesner and M. Pittelkow, Synlett., 2016, 498.

20. N. N. Karaush, G. V. Baryshnikov, V. A. Minaeva, H. Ågren and B. F. Minaev, Mol. Phys., 2017, 115, 2218.

21. N. N. Karaush-Karmazin, G. V. Baryshnikov, H. Agren and B. F. Minaev, In Reference Module in Chemistry, Molecular Sciences and Chemical Engineering, ed. H. N. C. Wong, Chapter Furans and Their Benzo Derivatives: Structure, Elsevier, 2019, 1.

22. G. V. Baryshnikov, R. R. Valiev, B. F. Minaev, H. Ågren, New J. Chem., 2017, 41, 2717.

23. V. A Minaeva, N. N. Karaush, B. F. Minaev, G. V. Baryshnikov, F. Chen, T. Tanaka and A. Osuka, Opt. Spectrosc., 2017, 122, 523.

24. N. N. Karaush, G. V. Baryshnikov, H. Ågren and B. F. Minaev, New J. Chem., 2018, 42, 11493. 
25. R. R. Valiev, V. N. Cherepanov, G. V. Baryshnikov and D. Sundholm, Phys. Chem. Chem. Phys., 2018, 20, 6121.

26. V. Minaeva, N. Karaush-Karmazin, G. Baryshnikov and B. Minaev, Vib. Spectrosc., 2019, 100, 107.

27. G. Baryshnikov, R. R. Valiev, V. N. Cherepanov, N. N. Karaush-Karmazin, V. A. Minaeva, B. F. Minaev, H. Ågren, Phys. Chem. Chem. Phys., 2019, 21, 9246.

28. C. B. Nielsen, T. Brock-Nannestad, T. K. Reenberg, P. Hammershøj, J. B. Christensen, J. W. Stouwdam and M. Pittelkow, Chem. Eur. J., 2010, 16, 13030 .

29. G. V. Baryshnikov, R. R. Valiev, N. N. Karaush, V. A. Minaeva, A. N. Sinelnikov, S. K. Pedersen, M. Pittelkow, B. F. Minaev and H. Ågren, Phys. Chem. Chem. Phys., 2016, 18, 28040.

30. K. B. Ivaniuk, G. V. Baryshnikov, P. Y. Stakhira, S. K. Pedersen, M. Pittelkow, A. Lazauskas, D. Volyniuk, J. V. Grazulevicius, B. F. Minaev and H. Ågren, J. Mater. Chem. C, 2017, 5, 4123.

31. T. Fujimoto, M. M. Matsushita and K. Awaga, J. Phys. Chem. C, 2012, 116, 5240.

32. T. Fujimoto, M. M. Matsushita and K. Awaga, Appl. Phys. Lett., 2010, 97, 123303.

33. A. Dadvand, F. Cicoira, K. Yu. Chernichenko, E. S. Balenkova, R. M. Osuna, F. Rosei, V. G. Nenajdenko and D. F. Perepichka, Chem. Commun., 2008, 5354.

34. G. Baryshnikov, B. Minaev, N. Karaush and V. Minaeva, Phys. Chem. Chem. Phys., 2014, 16, 6555.

35. G. Baryshnikov, B. Minaev, N. Karaush and V. Minaeva, RSC Adv., 2014, 4, 25843.

36. J. Yu, Q. Sun, Y. Kawazoe and P. Jena, Nanoscale, 2014, 6, 14962.

37. A. V. Kuklin, G. V. Baryshnikov, B. F. Minaev, N. Ignatova and H. Ågren, J. Phys. Chem. C, 2018, 122, 22216. 
38. N. N. Karaush, G. V. Baryshnikov and B. F. Minaev, RSC Adv., 2015, 5, 24299.

39. N. N. Karaush, G. V. Baryshnikov, B. A. Minaeva and B. F. Minaev, New J. Chem., 2015, 39, 7815.

40. X. Xiong, C.-L. Deng, B. F. Minaev, G. V. Baryshnikov, X.-S. Peng and H. N. C. Wong, Chem. Asian. J., 2015, 10, 969.

41. V. A. Minaeva, G. V. Baryshnikov, B. F. Minaev, N. N. Karaush, X.-D. Xiong, M.-D. Li, D. L. Phillips and H. N. C. Wong, Spectrochim. Acta A Mol. Biomol. Spectrosc., 2015, 151, 247.

42. G. V. Baryshnikov, N. N. Karaush, R. R. Valiev, B. F. Minaev, J. Mol. Model., 2015, 21, 136.

43. V. T. T. Huong, T. B. Tai and M. T. Nguyen, RSC Adv., 2015, 5, 24167.

44. S. Kato, S. Akahori, Y. Serizawa, X. Lin, M. Yamauchi, S. Yagai, T. Sakurai, W. Matsuda, S. Seki, H. Shinokubo and Y. Miyake, J. Org. Chem., 2020, 85, 62.

45. A. D. Becke, J. Chem. Phys., 1993, 98, 5648.

46. C. Lee, W. Yang and R. G. Parr, Phys. Rev. B, 1988, 37, 785.

47. M. S. Gordon, J. S. Binkley, J. A. Pople, W. J. Pietro and W. J. Hehre, J. Am. Chem. Soc., 1982, 104, 2797.

48. M. M. Francl, W. J. Pietro, W. J. Hehre, J. S. Binkley, D. J. DeFrees, J. A. Pople and M. S. Gordon, J. Chem. Phys., 1982, 77, 3654.

49. R. Krishnan, J. S. Binkley, R. Seeger and J. A. Pople, J. Chem. Phys., 1980, 72, 650.

50. M. J. Frisch, G. W. Trucks, H. B. Schlegel, G. E. Scuseria, M. A. Robb, J. R. Cheeseman, G. Scalmani, V. Barone, B. Mennucci, G. A. Petersson, H. Nakatsuji, M. Caricato, X. Li, H. P. Hratchian, A. F. Izmaylov, J. Bloino, G. Zheng,J. L. Sonnenberg, M. Hada, M. Ehara, K. Toyota, R. Fukuda, J. Hasegawa, M. Ishida, T. Nakajima, Y. Honda, O. Kitao, H. Nakai, T. Vreven, J. A. Montgomery, Jr., J. E. Peralta, F. Ogliaro, M. Bearpark, J. J. Heyd, E. Brothers, K. N. Kudin, V. N. Staroverov, R. Kobayashi, J. Normand, K. 
Raghavachari, A. Rendell, J. C. Burant, S. S. Iyengar, J. Tomasi, M. Cossi, N. Rega, J. M. Millam, M. Klene, J. E. Knox, J. B. Cross, V. Bakken, C. Adamo, J. Jaramillo, R. Gomperts, R. E. Stratmann, O. Yazyev, A. J. Austin, R. Cammi, C. Pomelli, J. W. Ochterski, R. L. Martin, K. Morokuma, V. G. Zakrzewski, G. A. Voth, P. Salvador, J. J. Dannenberg, S. Dapprich, A. D. Daniels, O.Ë. Farkas, J. B. Foresman, J. V. Ortiz, J. Cioslowski and D. J. Fox, Gaussian 16, Rev. A. 03, Gaussian, Inc., Wallingford, CT, 2016.

51. E. Runge and E. K. U. Gross, Phys. Rev. Lett., 1984, 52, 997.

52. G. Kresse and J. Furthmüller, Phys. Rev. B, 1996, 54, 11169.

53. G. Kresse and J. Hafner, Phys. Rev. B, 1993, 47, 558.

54. P. E. Blöchl, Phys. Rev. B, 1994, 50, 17953.

55. J. P. Perdew, K. Burke and M. Ernzerhof, Phys. Rev. Lett., 1996, 77, 3865.

56. J. Heyd, G. E. Scuseria and M. Ernzerhof, J. Chem.Phys., 2003, 118, 8207.

57. H. J. Monkhorst and J. D. Pack, Phys. Rev. B, 1976, 13, 5188.

58. J. Yu, Q. Sun, P. Jena, J. Phys. Chem. C, 2016, 120, 49, 27829.

59. A. Tsuda and A. Osuka, Science, 2001, 293, 79.

60. L. Liua and Z. Shen, Appl. Phys. Lett., 2009, 95, 252104.

61. P. Ruffieux, J. Cai, N. C. Plumb, L. Patthey, D. Prezzi, A. Ferretti, E. Molinari, X. Feng, K. Müllen, C. A. Pignedoli and R. Fasel, ACS Nano, 2012, 6, 6930.

62. N. N. Karaush, S. V. Bondarchuk, G. V. Baryshnikov, V. A. Minaeva, W.H. Sun and B. F. Minaev, RSC Adv., 2016, 6, 49505.

63. R. Gutzler and D. F. Perepichka, J. Am. Chem. Soc., 2013, 135, 16585.

64. O. Loboda, I. Tunell, B. Minaev and H. Ågren, Chem. Phys., 2005, 312, 299.

65. B. F. Minaev, S. Knuts and H. Ågren, Chem. Phys., 1994, 181, 15. 

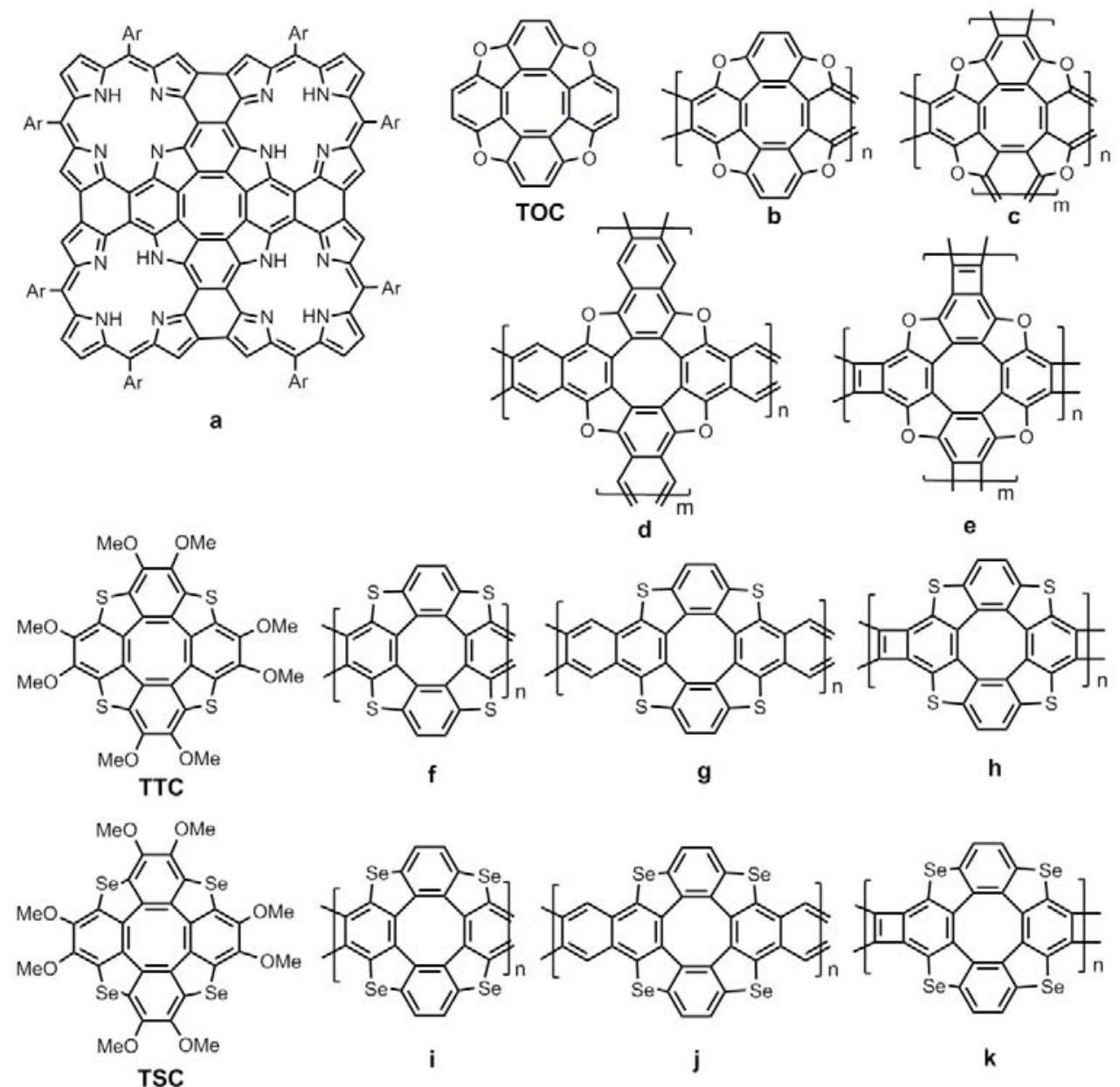

Some examples of the 1D and 2D polymers containing porphyrin (a),9, 10 tetraoxa[8]circulene (b-e),34-37 tetrathia[8]circulene $(\mathrm{f}-\mathrm{h})$ and tetraselena[8]circulene $(\mathrm{i}-\mathrm{k})$ monomers studied in this paper. 


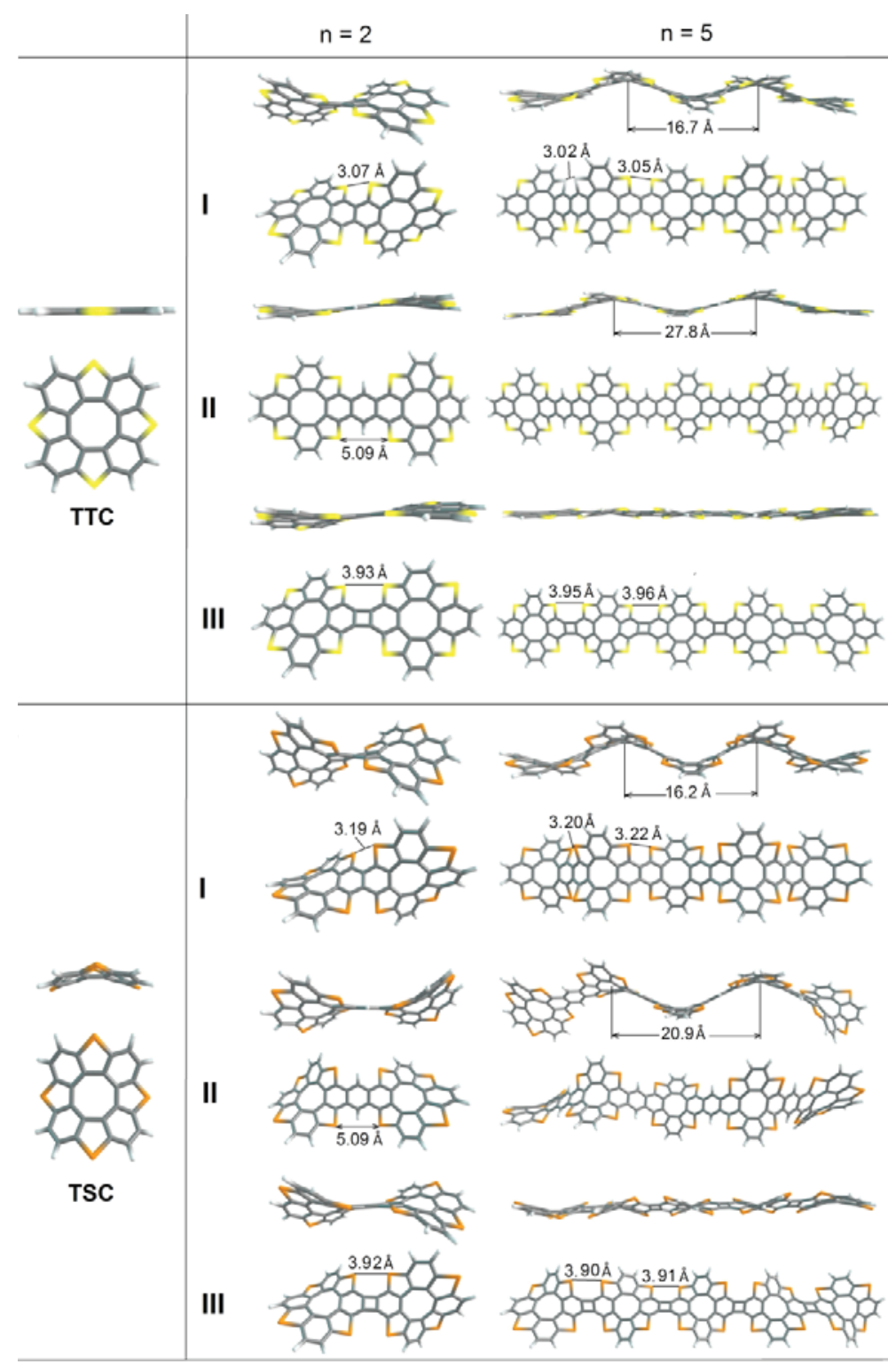

Molecular representations of the TTC and TSC structures with $n=2$ and $n=5$ (side and top view). $189 \times 293 \mathrm{~mm}(300 \times 300 \mathrm{DPI})$ 

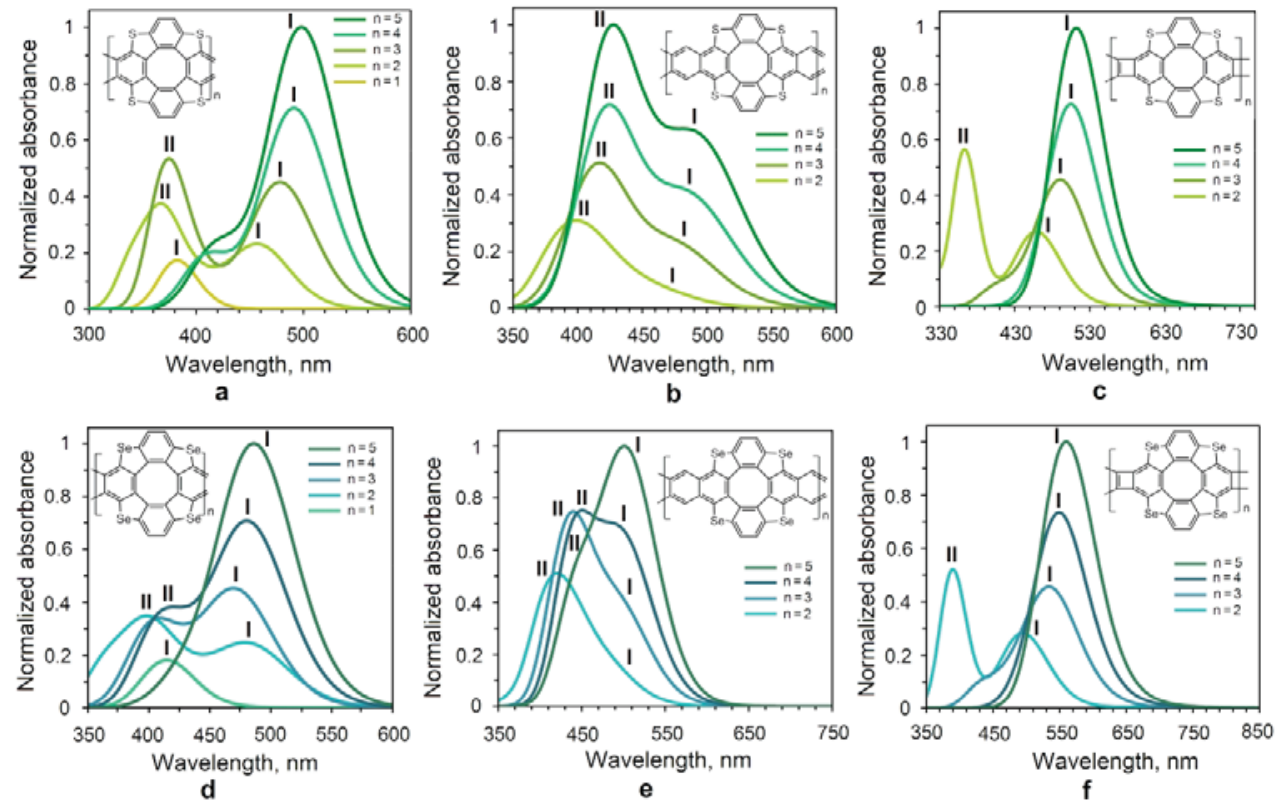

Electronic absorption spectra of the TTC- and TSC-based ribbons calculated by the TD DFT/B3LYP/6-31G(d) method; I and II denote the first and second absorption bands (band half-width $3000 \mathrm{~cm}-1$, Gaussian distribution function). 


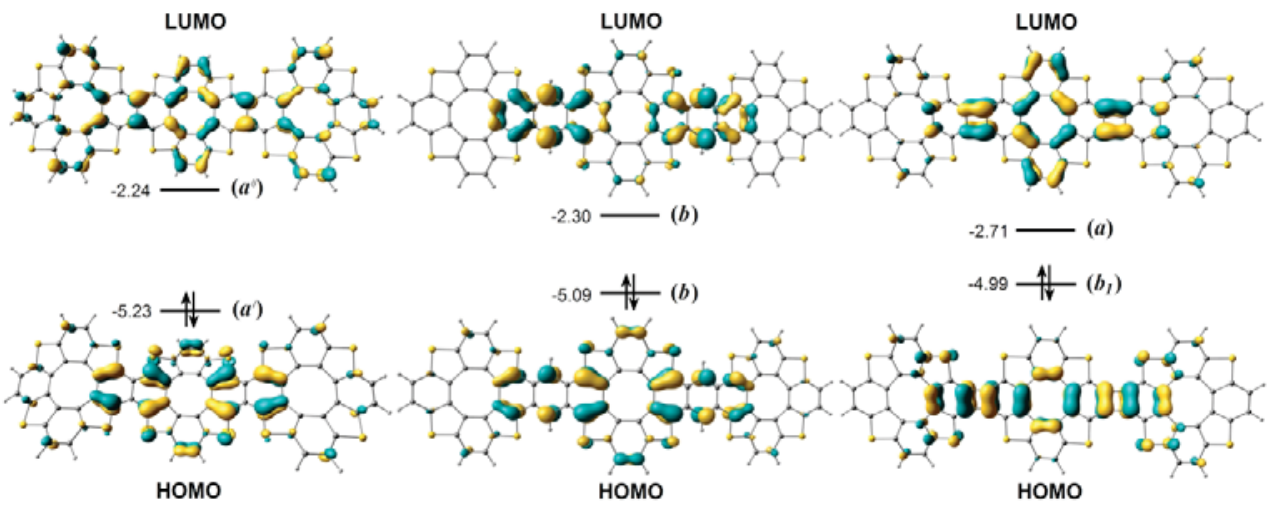

Simplified molecular orbital energy diagram for the TTC ribbons of different fusion types with $n=3$. (For the TSC ribbons with $n=3$ the MOs are identical). With increasing TTC/TSC units ( $n$ ) MOs have the same nature for the corresponding TTC/TSC ribbon type. 

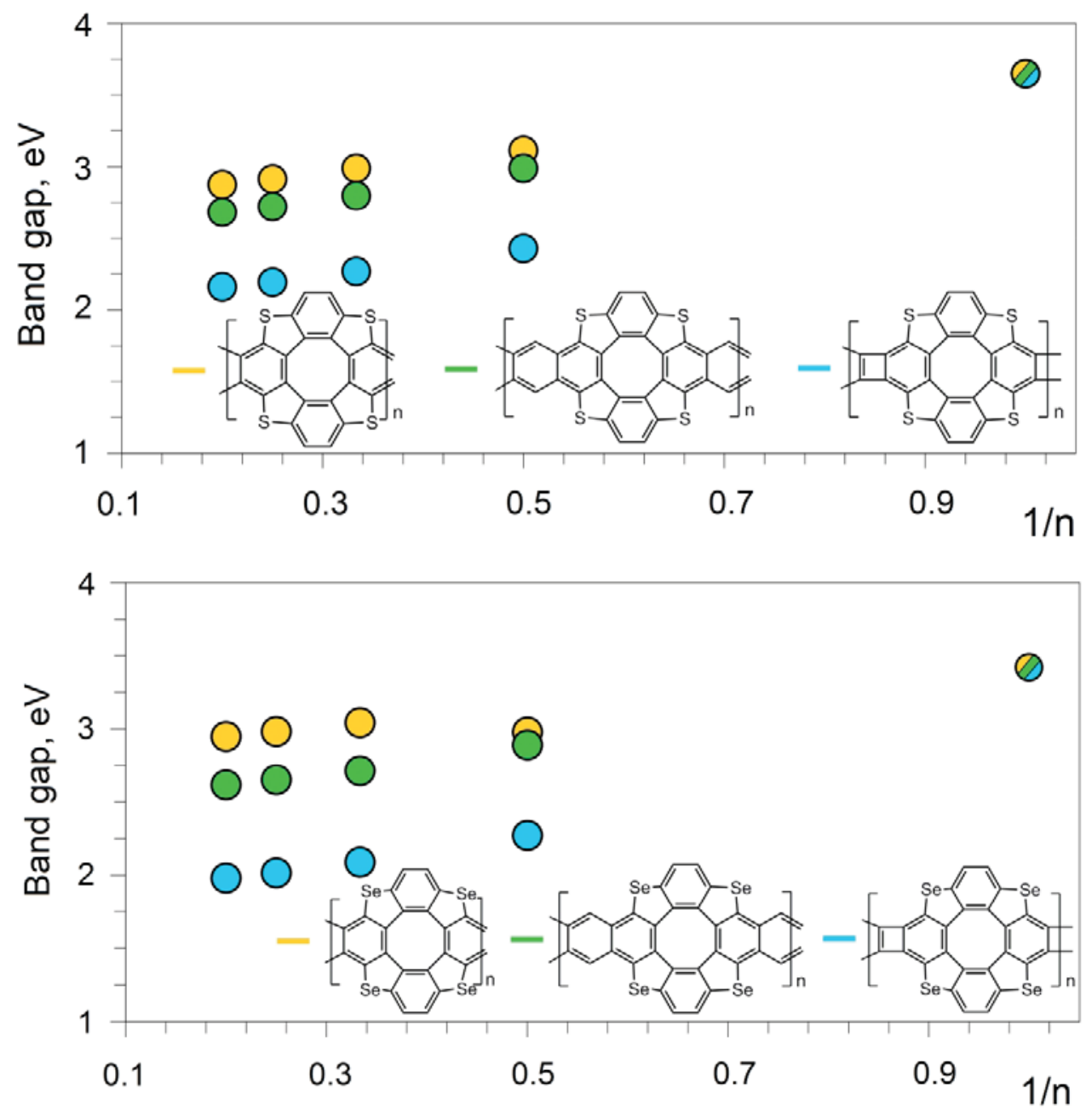

The length dependence behaviour of the band gap for the TTC- and TSC-based 1D oligomers ( $\mathrm{n}$ denote the numbers of the TTC and TSC monomers). Yellow, green and blue colors correspond to I, II and III types of ribbons. 


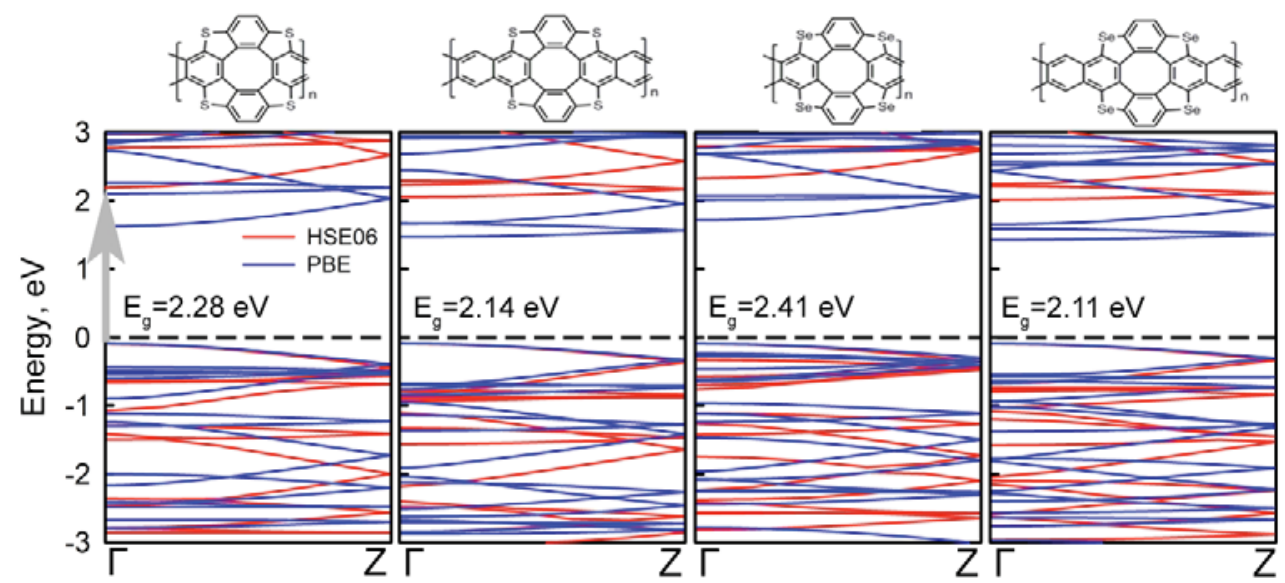

Band structures of the TTC- and TSC-based 1D polymers calculated at the PBE (blue) and HSE06 (red) levels of theory. The Fermi level is set to $0 \mathrm{eV}$. 


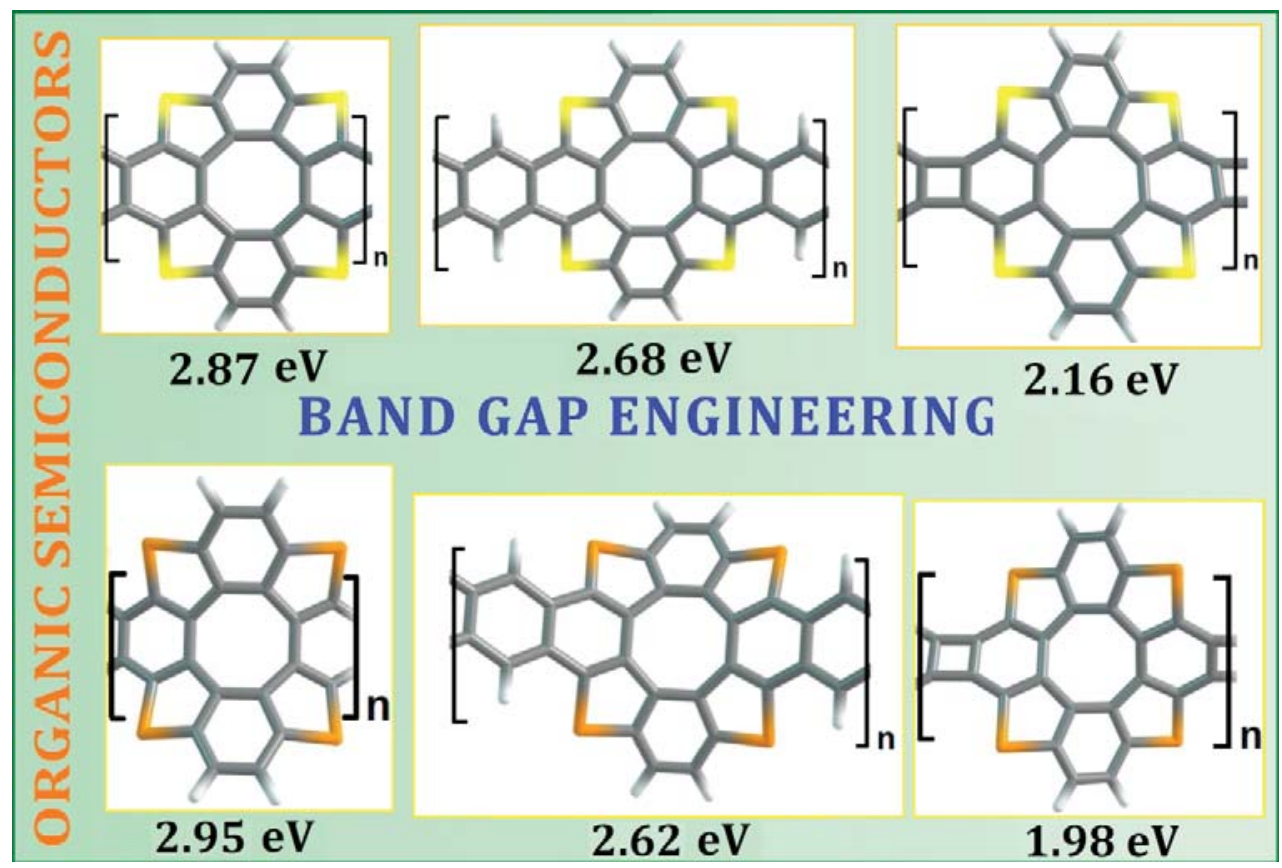




\section{Structure, Stability and Electronic Properties of One-Dimensional Tetrathia- and Tetraselena[8]circulene-based Materials: A Comparative DFT Study}

Nataliya N. Karaush-Karmazin *,a, Artem V. Kuklin*,b, Gleb V. Baryshnikov ${ }^{a, c}$, Lyudmila V. Begunovich ${ }^{b}$, Hans Ågren ${ }^{c, d}$ and Boris F. Minaev ${ }^{a}$

${ }^{a}$ Department of Chemistry and Nanomaterials Science, Bohdan Khmelnytsky National University, 18031, Cherkasy, Ukraine ${ }^{b}$ Department of Science and Innovations, Siberian Federal University, 79

Svobodny Av., Krasnoyarsk, 660041, Russian Federation

${ }^{c}$ Division of Theoretical Chemistry and Biology, School of Engineering Sciences in Chemistry, Biotechnology and Health, KTH Royal Institute of Technology, 10691

Stockholm, Sweden

${ }^{d}$ College of Chemistry and Chemical Engineering, Henan University, Kaifeng, Henan 475004 P. R. China

\section{Electronic Supplementary Information}

\section{List of Content}

\begin{tabular}{|l|c|}
\hline Figures S1-12. MOs diagram of the tetrathia[8]circulene-based compound $(\mathrm{n}=2-5)$ & $\mathbf{2}$ \\
\hline Figures S13-18. MOs diagram of the tetraselena[8]circulene-based compound $(\mathrm{n}=2$ and 3$)$ & $\mathbf{1 3}$ \\
\hline $\begin{array}{l}\text { Table S1. Wavelengths, oscillator strengths and orbital assignment of the selected } \\
\text { electronic transitions in the calculated absorption spectra of the TTC-based oligomers }\end{array}$ & $\mathbf{1 6}$ \\
\hline $\begin{array}{l}\text { Table S2. Wavelengths }(\lambda) \text {, oscillator strengths }(f) \text { and orbital assignment of the selected } \\
\text { electronic transitions in the calculated absorption spectra of the TSC-based oligomers }\end{array}$ & $\mathbf{2 2}$ \\
\hline Optimized Cartesian coordinates for selected compounds (Tables S3-S28) & $\mathbf{2 8}$ \\
\hline
\end{tabular}

*Corresponding authors: N. Karaush-Karmazin karaush22@ukr.net and Artem Kuklin artem.icm@gmail.com. 


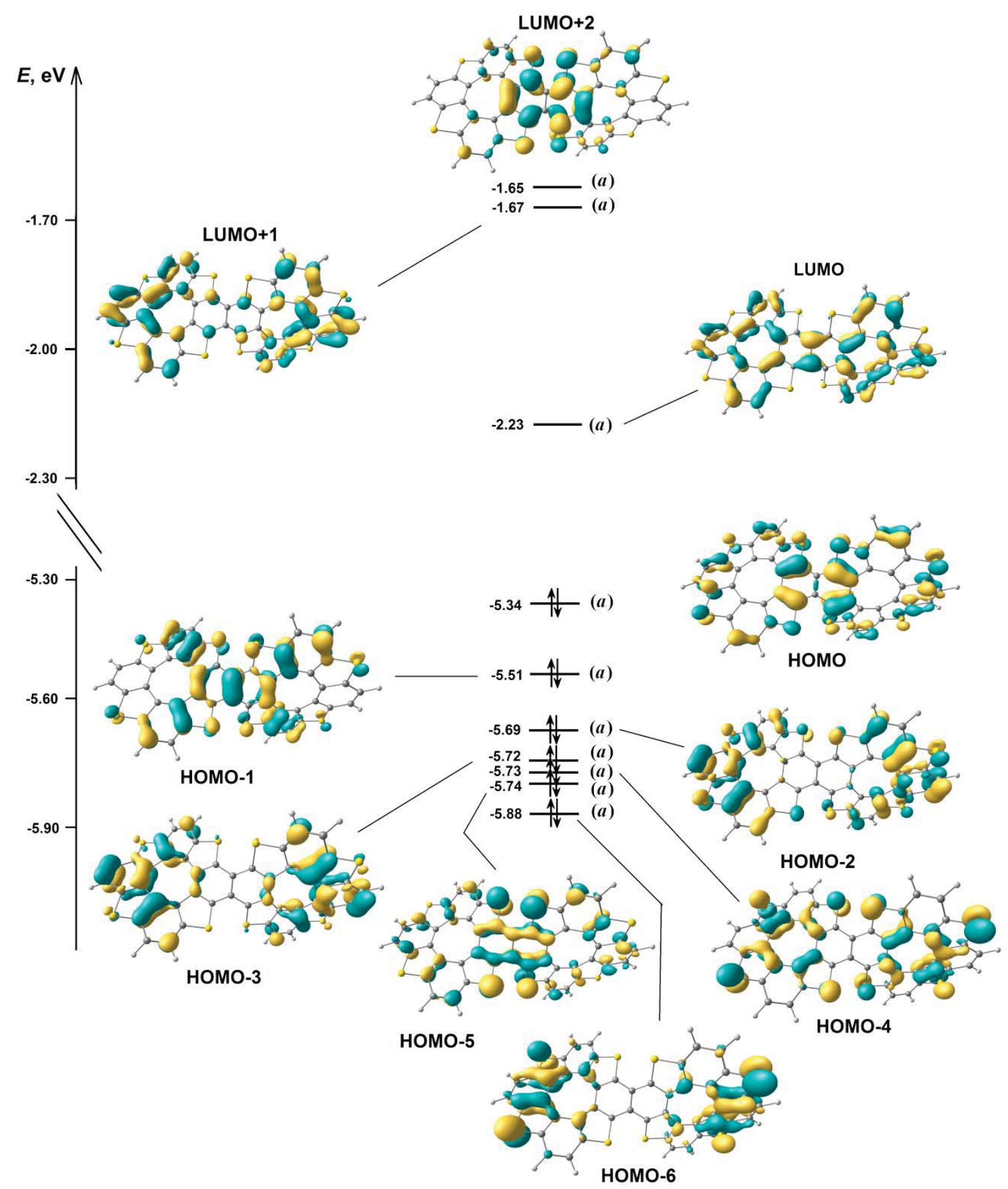

Figure S1. Molecular orbital diagram of the tetrathia[8]circulene-based compound with $n=2$ (type I) calculated at the B3LYP/6-31G(d) level of theory. 


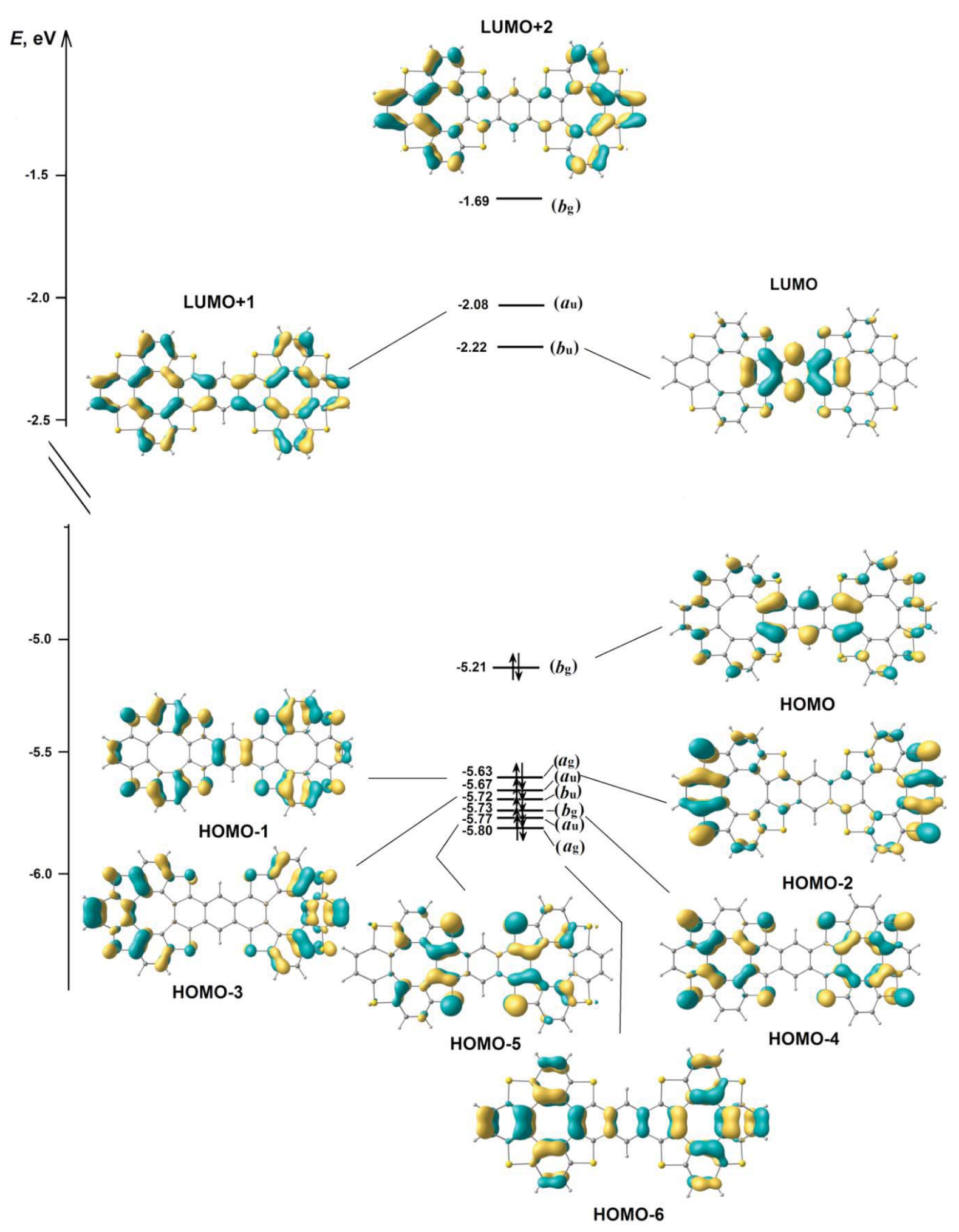

Figure S2. Molecular orbital diagram of the tetrathia[8]circulene-based compound with $n=2$ (type II) calculated at the B3LYP/6-31G(d) level of theory. 


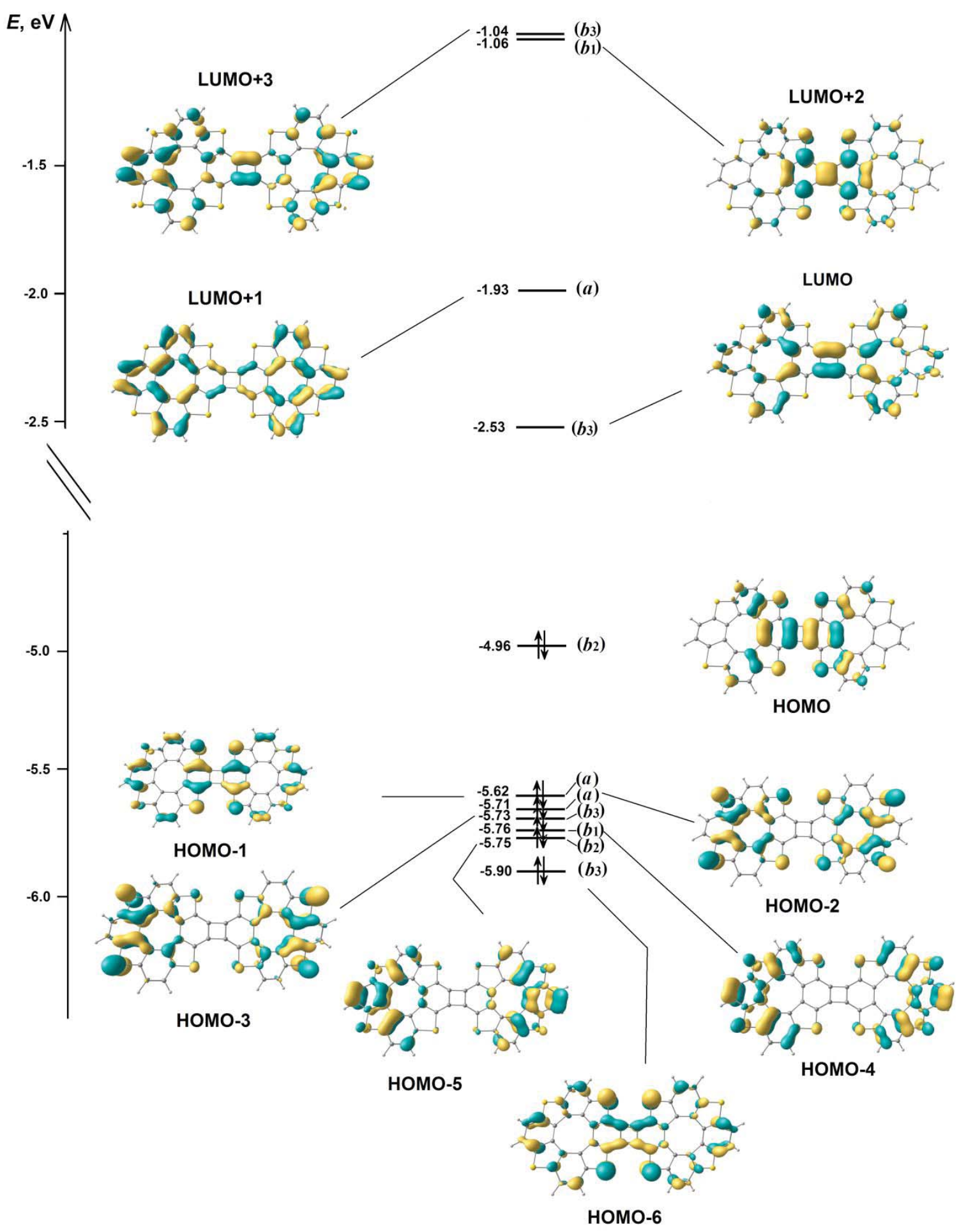

Figure S3. Molecular orbital diagram of the tetrathia[8]circulene-based compound with $n=2$ (type III) calculated at the B3LYP/6-31G(d) level of theory. 


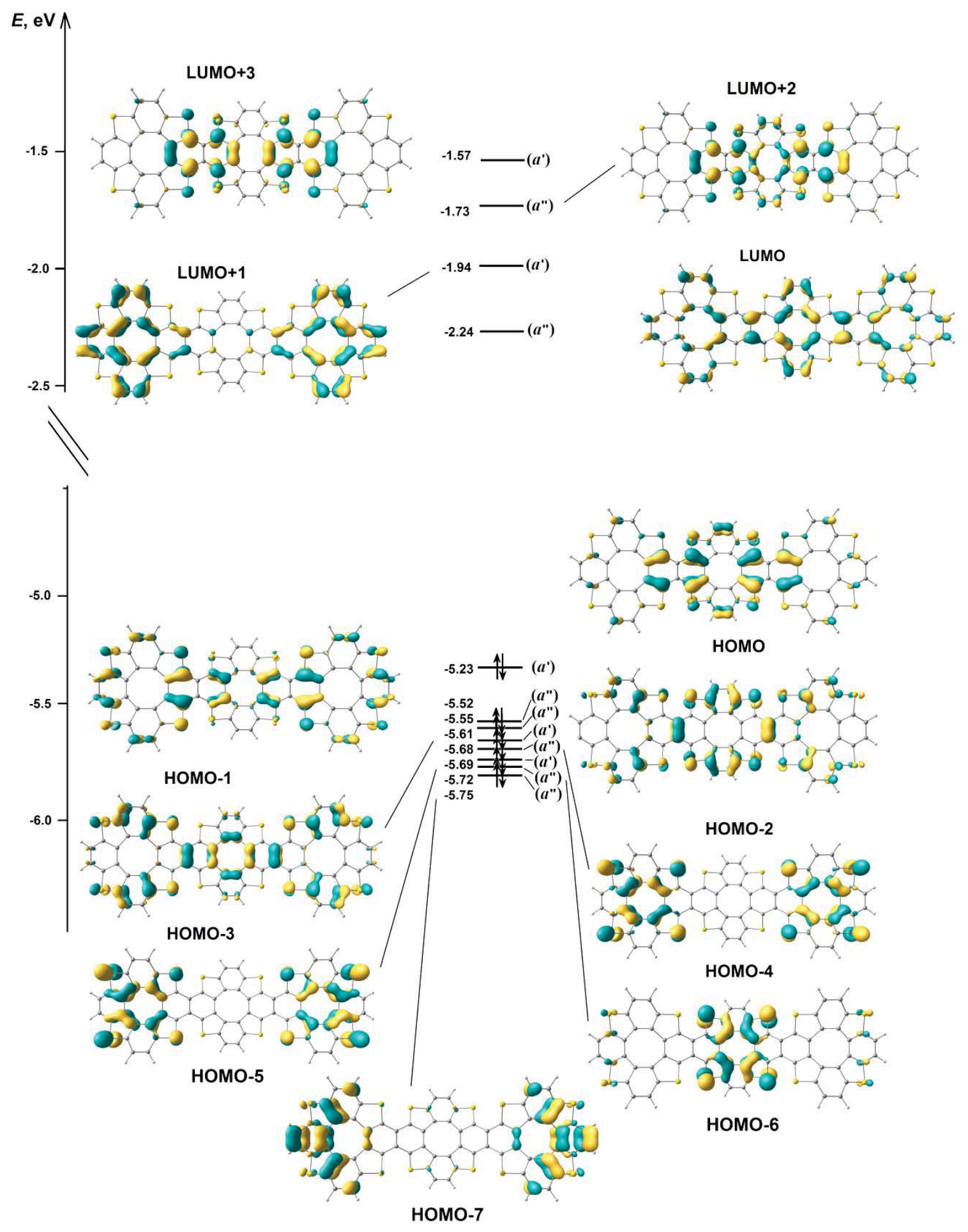

Figure S4. Molecular orbital diagram of the tetrathia[8]circulene-based compound with $n=3$ (type I) calculated at the B3LYP/6-31G(d) level of theory. 


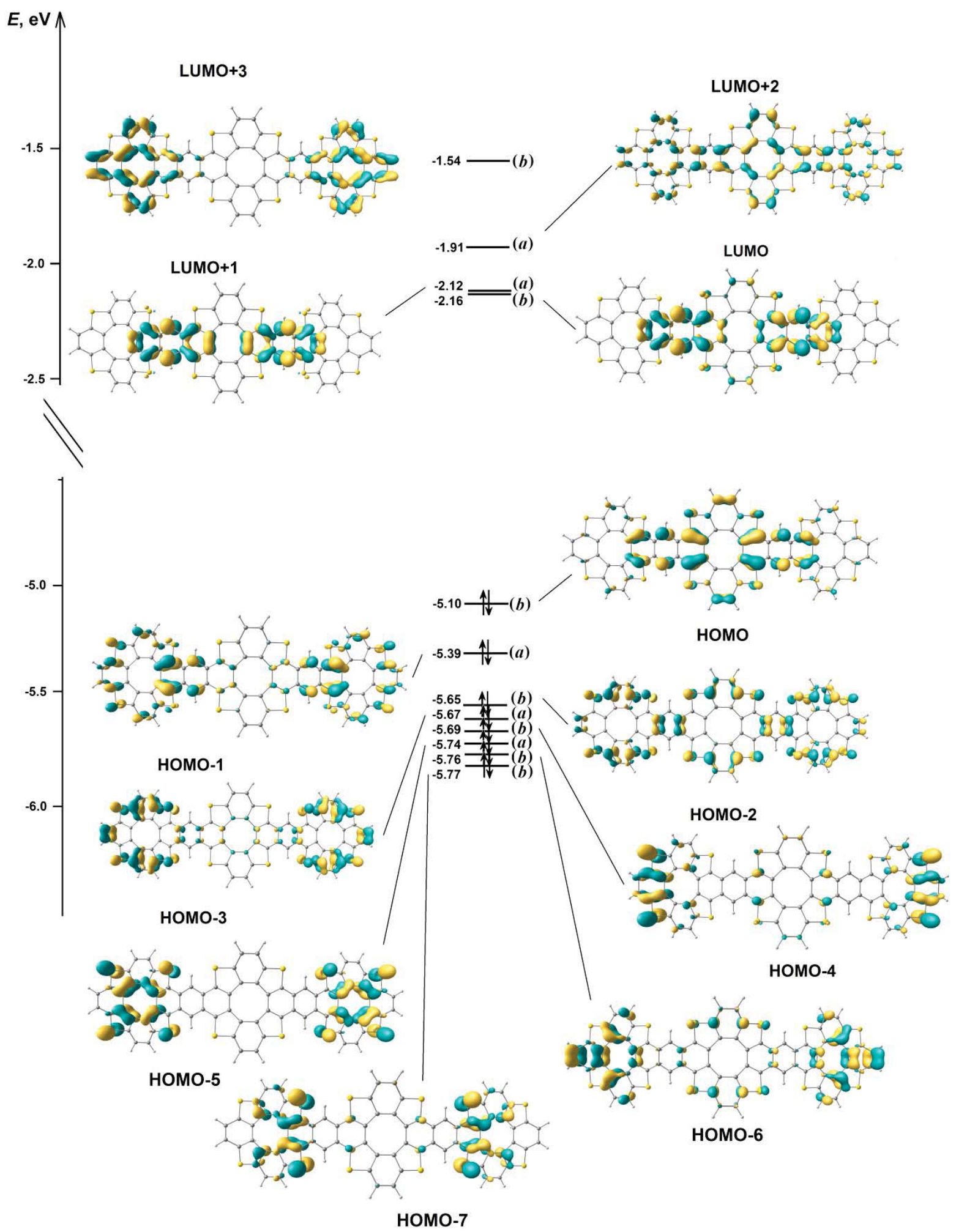

Figure S5. Molecular orbital diagram of the tetrathia[8]circulene-based compound with $n=3$ (type II) calculated at the B3LYP/6-31G(d) level of theory. 


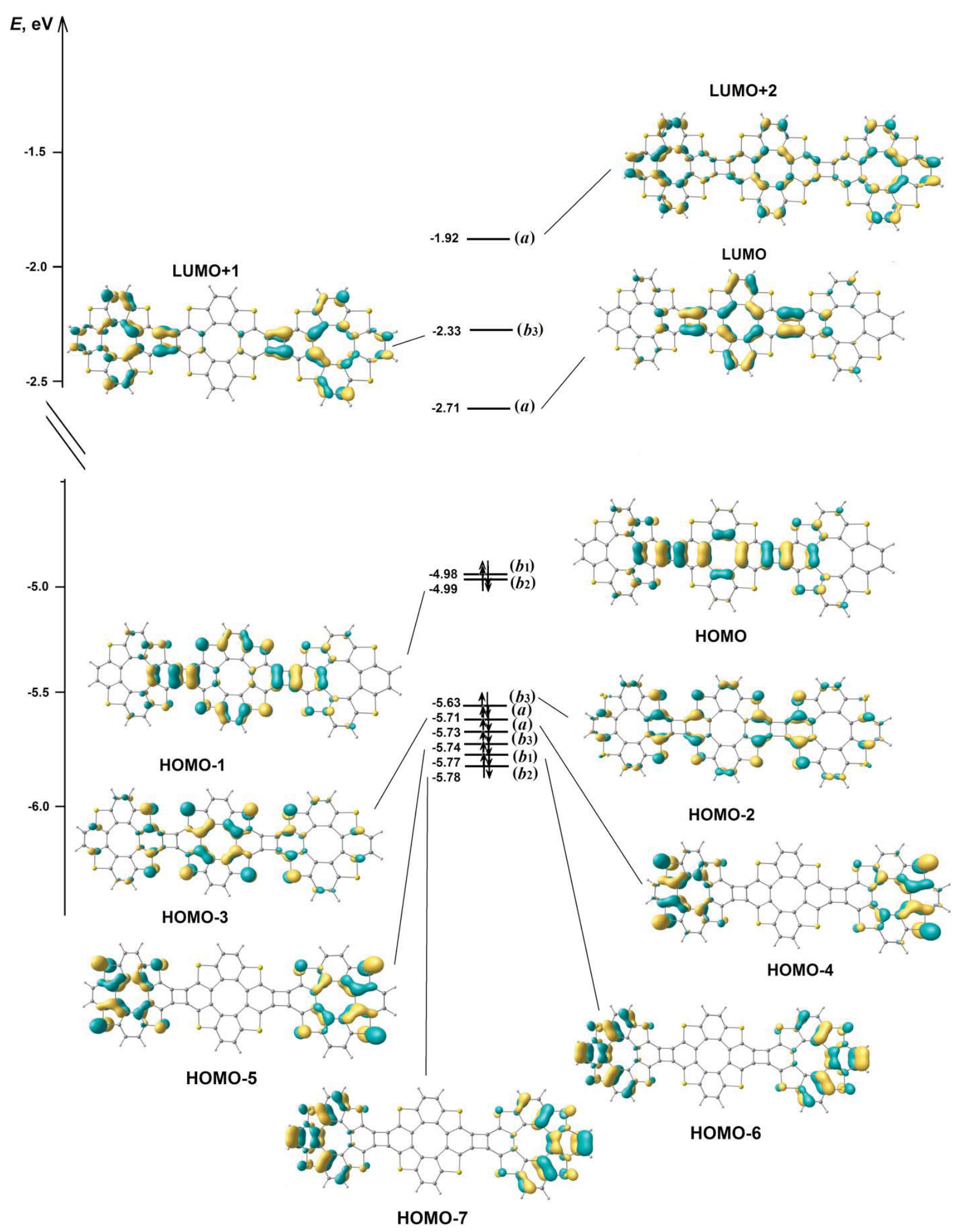

Figure S6. Molecular orbital diagram of the tetrathia[8]circulene-based compound with $n=3$ (type III) calculated at the B3LYP/6-31G(d) level of theory. 


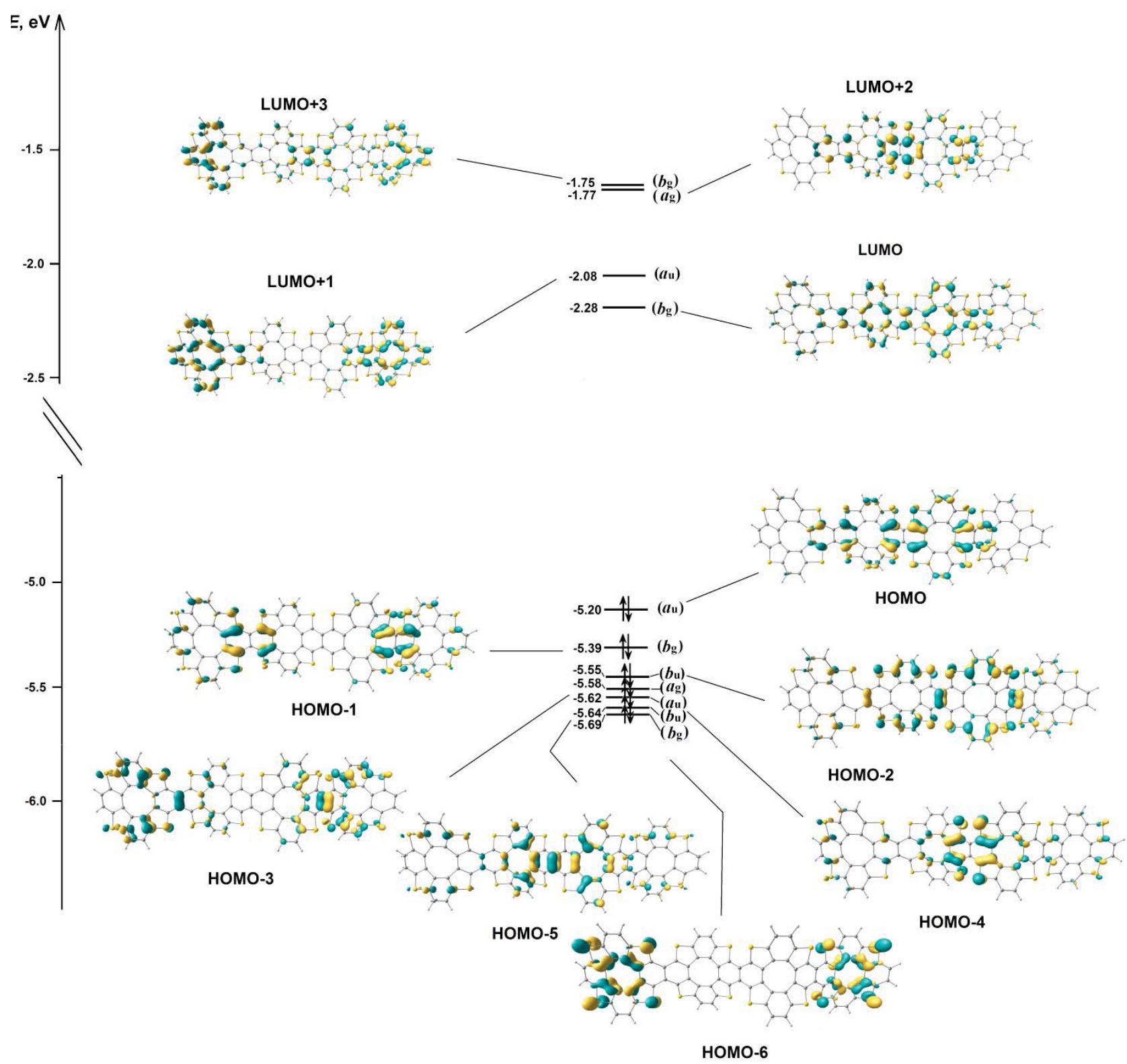

Figure S7. Molecular orbital diagram of the tetrathia[8]circulene-based compound with $n=4$ (type I) calculated at the B3LYP/6-31G(d) level of theory. 


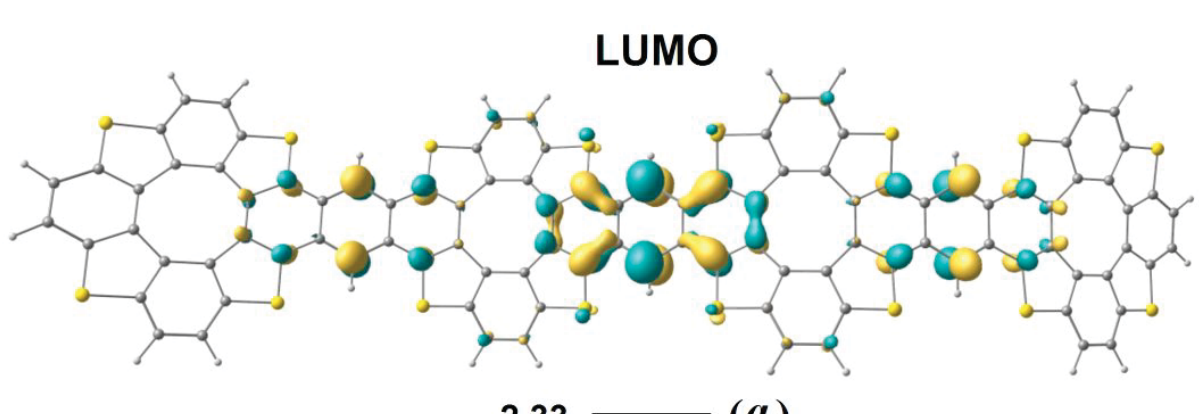

$-2.33-(a)$

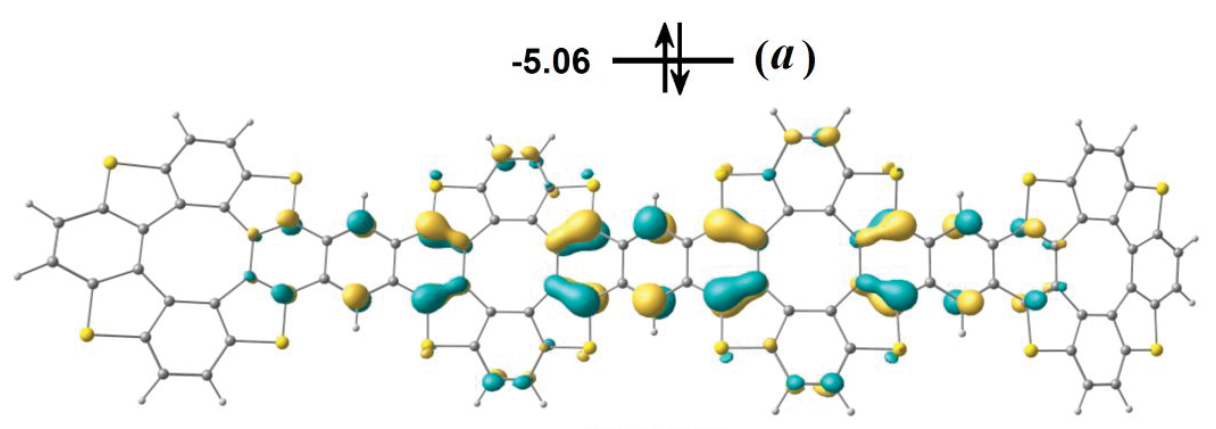

HOMO

Figure S8. Molecular orbital diagram of the tetrathia[8]circulene-based compound with $n=4$ (type II) calculated at the B3LYP/6-31G(d) level of theory. 


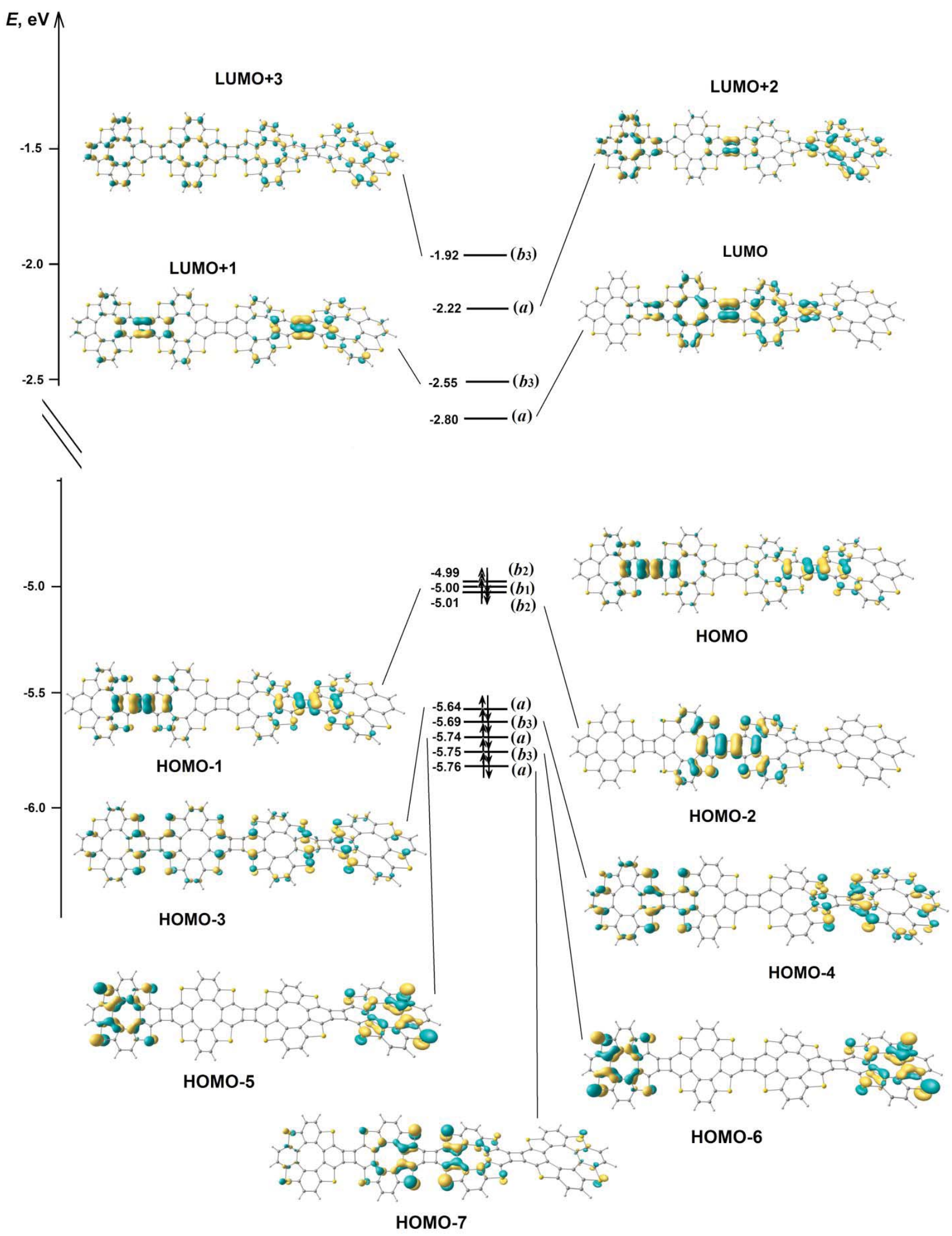

Figure S9. Molecular orbital diagram of the tetrathia[8]circulene-based compound with $n=4$ (type III) calculated at the B3LYP/6-31G(d) level of theory. 

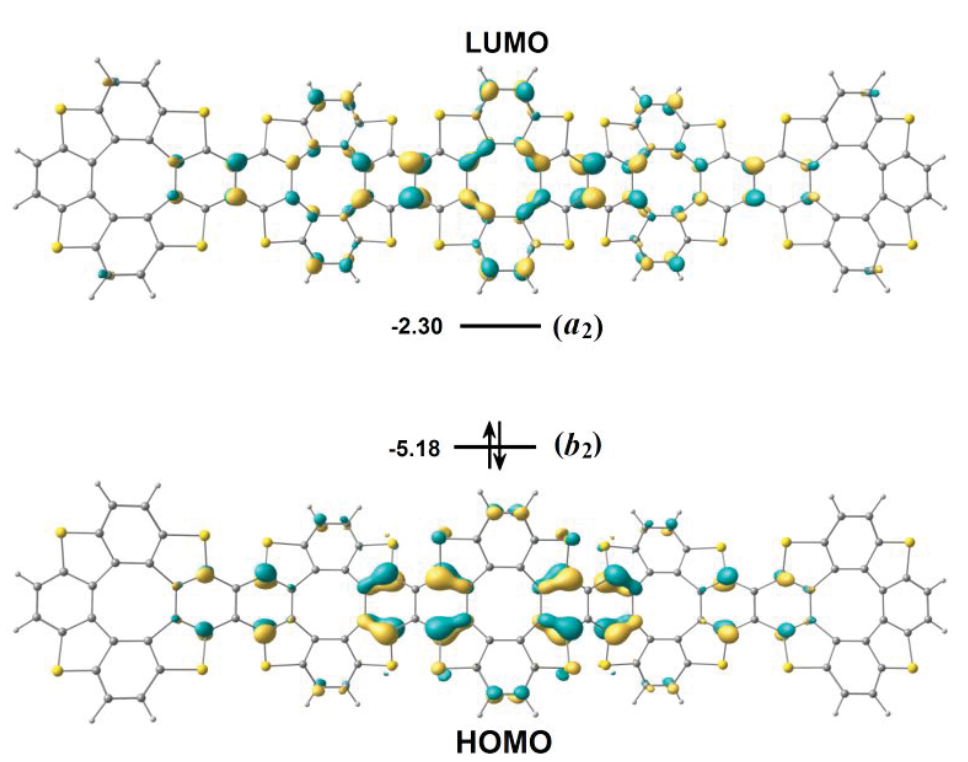

Figure S10. Molecular orbital diagram of the tetrathia[8]circulene-based compound with $n=5$ (type I) calculated at the B3LYP/6-31G(d) level of theory.

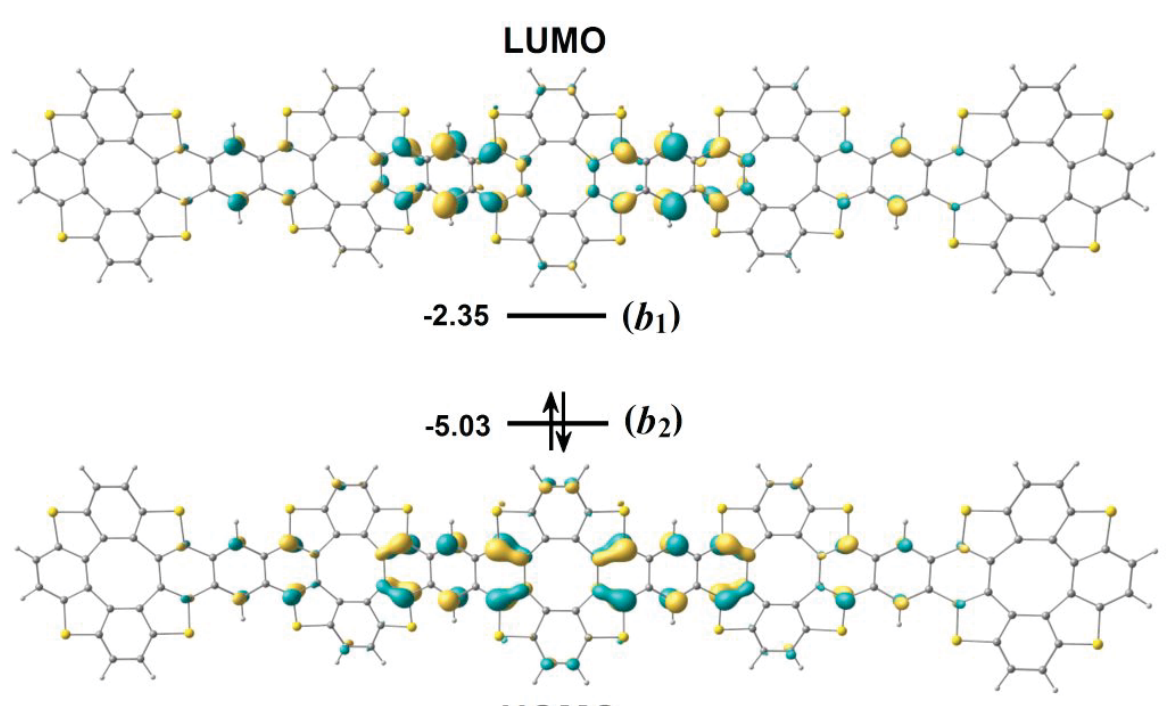

HOMO

Figure S11. Molecular orbital diagram of the tetrathia[8]circulene-based compound with $\mathrm{n}=5$ (type II) calculated at the B3LYP/6-31G(d) level of theory. 

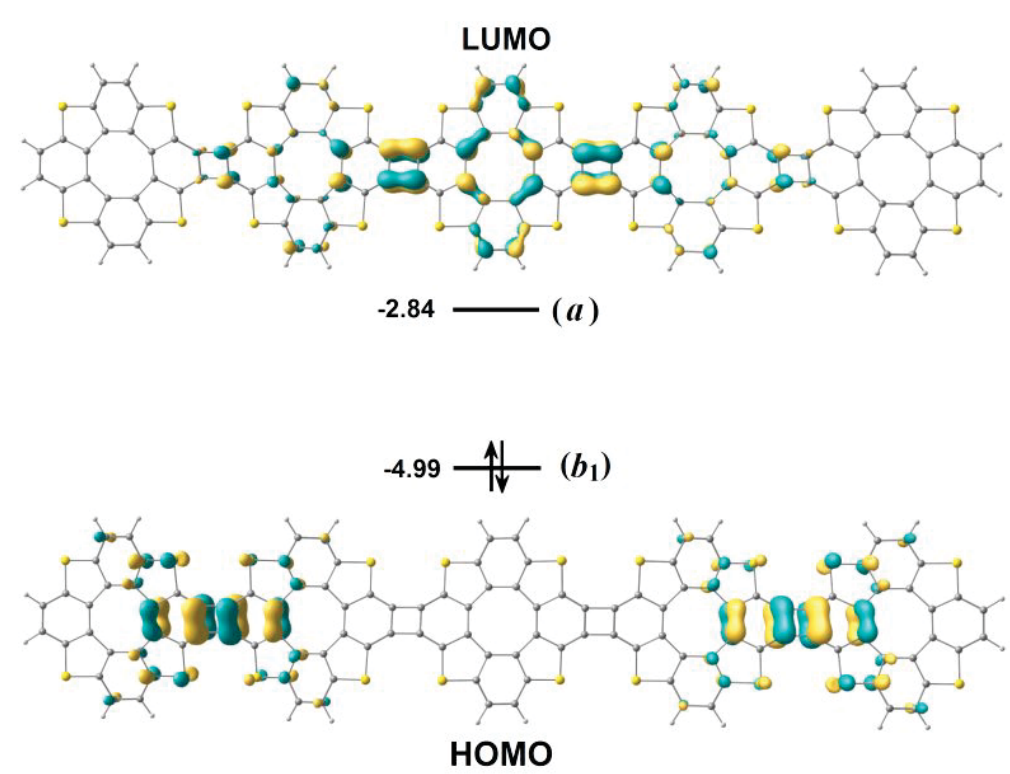

Figure S12. Molecular orbital diagram of the tetrathia[8]circulene-based compound with $n=5$ (type III) calculated at the B3LYP/6-31G(d) level of theory. 

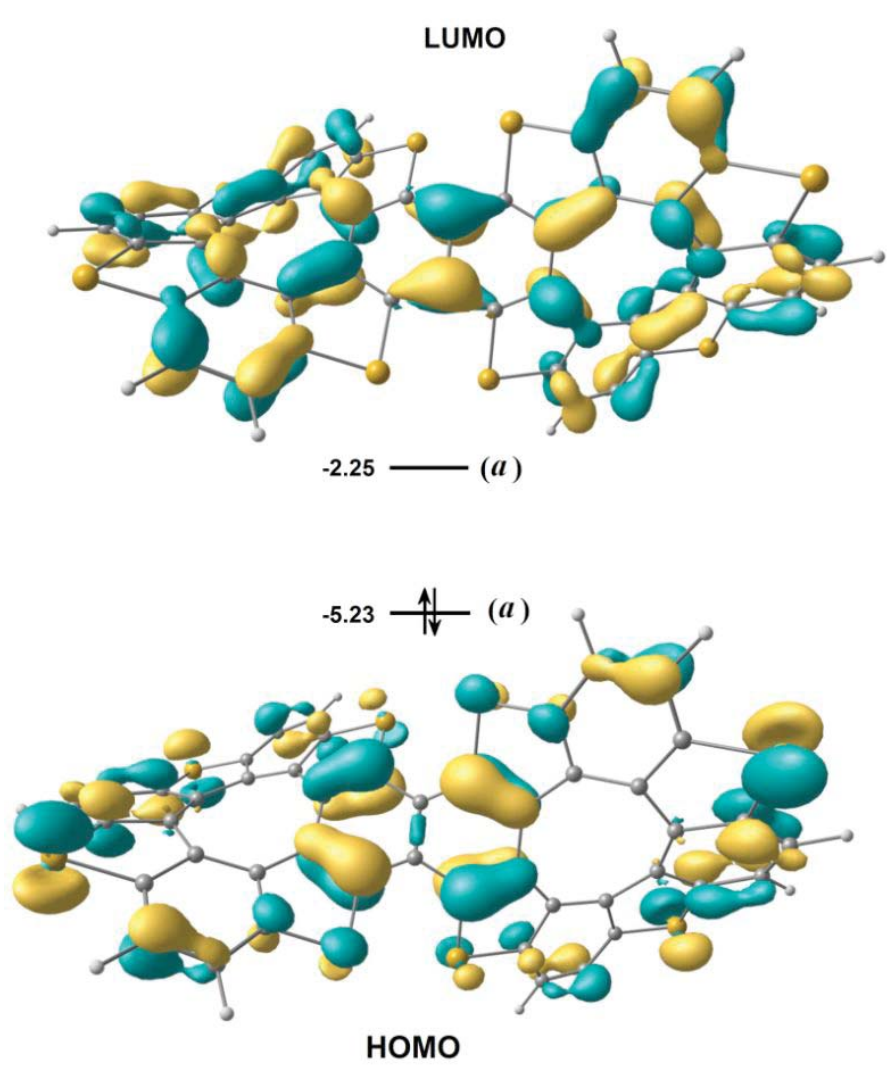

Figure S13. Molecular orbital diagram of the tetraselena[8]circulene-based compound with $n=2$ (type I) calculated at the B3LYP/6-31G(d) level of theory.
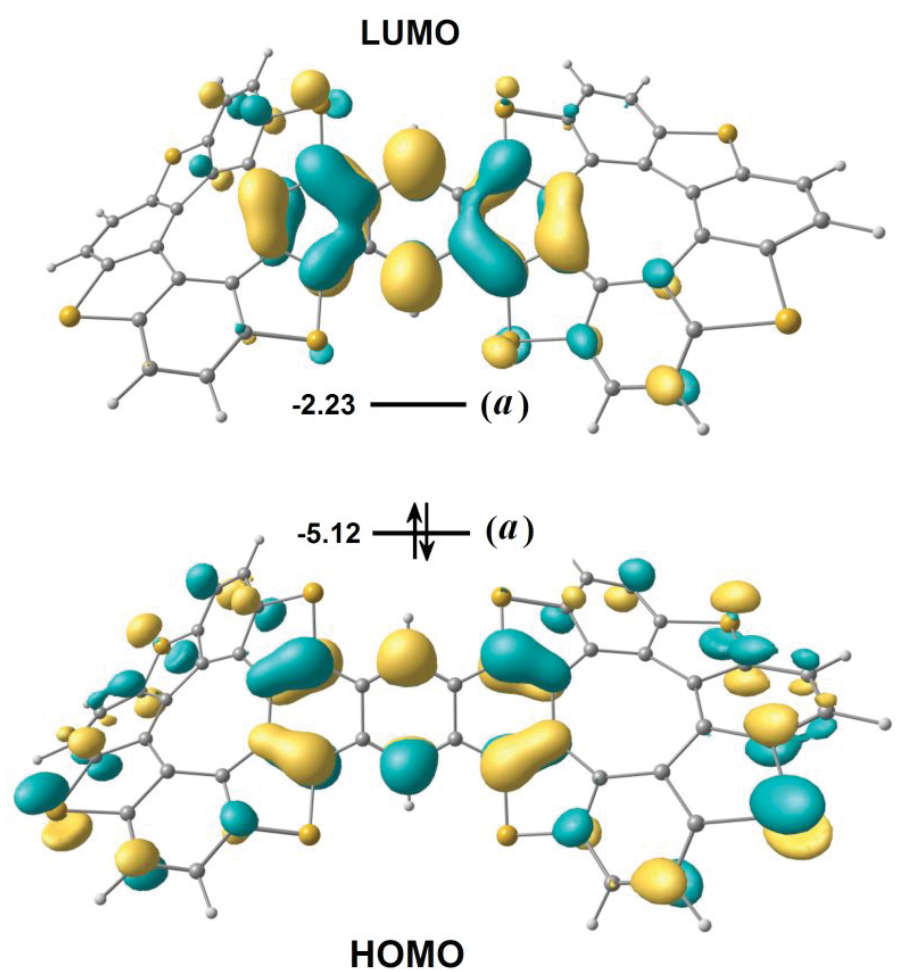

Figure S14. Molecular orbital diagram of the tetraselena[8]circulene-based compound with $\mathrm{n}=2$ (type II) calculated at the B3LYP/6-31G(d) level of theory. 

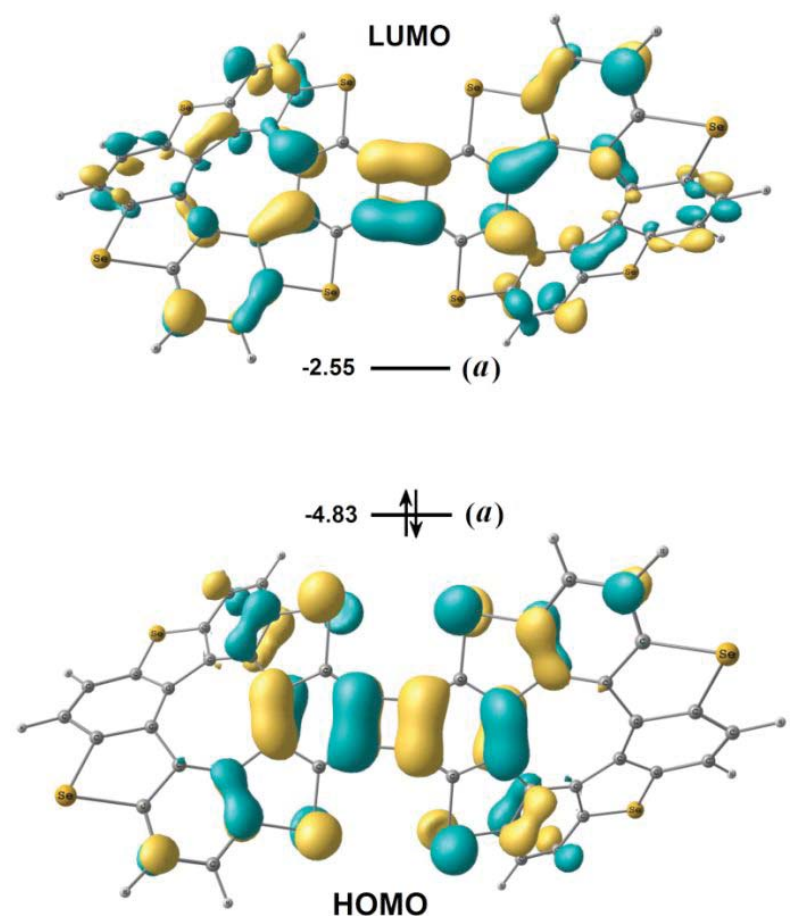

Figure S15. Molecular orbital diagram of the tetraselena[8]circulene-based compound with $\mathrm{n}=2$ (type III) calculated at the B3LYP/6-31G(d) level of theory.
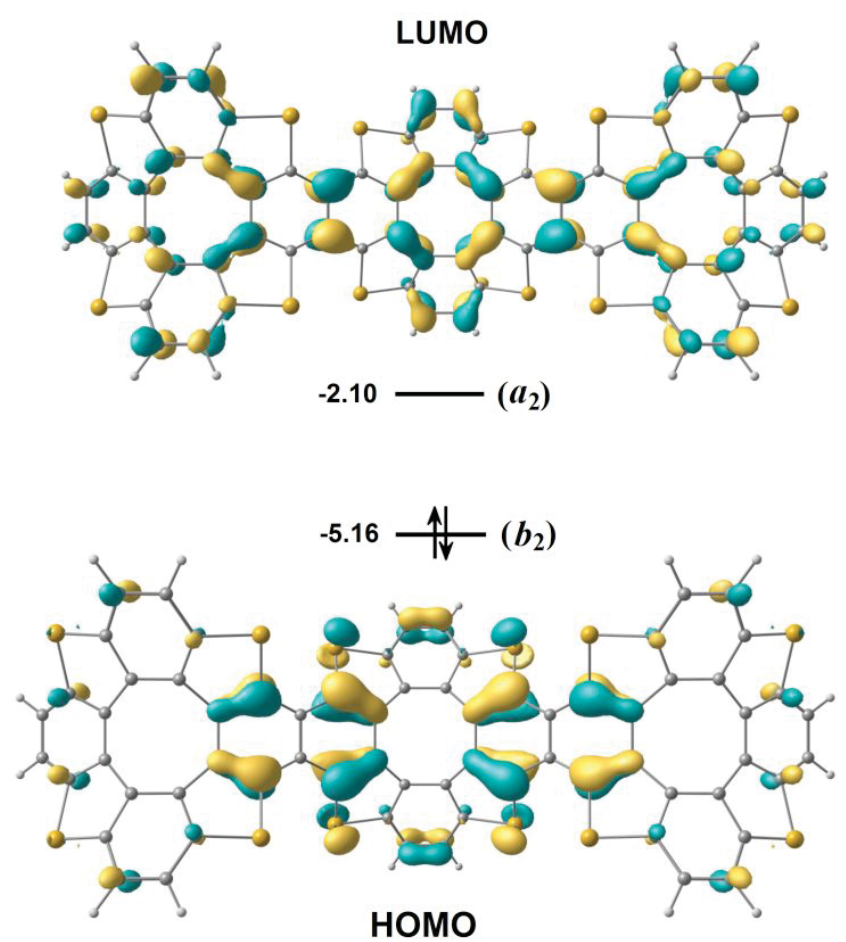

Figure S16. Molecular orbital diagram of the tetraselena[8]circulene-based compound with $n=3$ (type II) calculated at the B3LYP/6-31G(d) level of theory. 


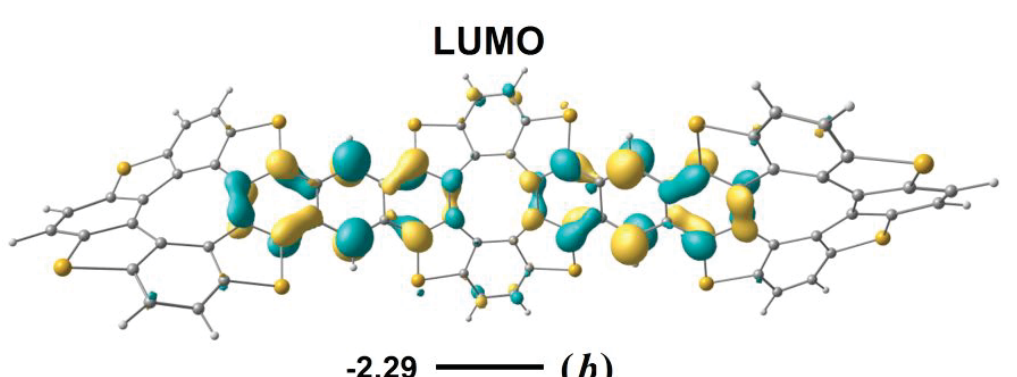

$-2.29-(b)$

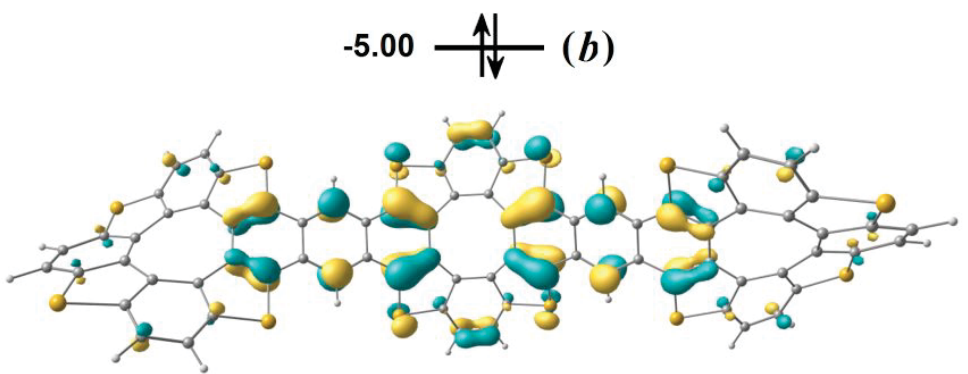

HOMO

Figure S17. Molecular orbital diagram of the tetraselena[8]circulene-based compound with $n=3$ (type II) calculated at the B3LYP/6-31G(d) level of theory.
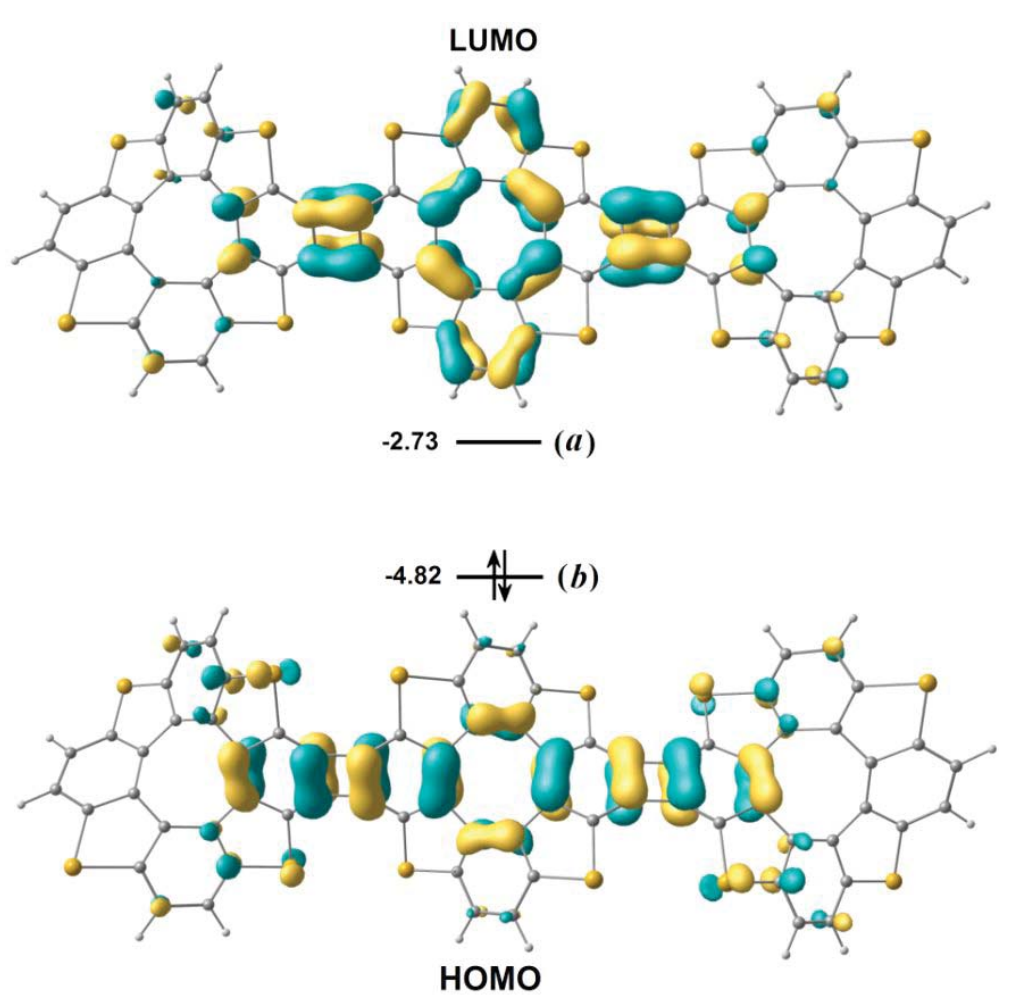

Figure S18. Molecular orbital diagram of the tetraselena[8]circulene-based compound with $\mathrm{n}=3$ (type III) calculated at the B3LYP/6-31G(d) level of theory. 
Table S1. Wavelengths $(\lambda)$, oscillator strengths $(f)$ and orbital assignment of the selected electronic transitions in the calculated absorption spectra of the TTC-based oligomers

\begin{tabular}{|c|c|c|c|c|c|}
\hline Compound & State & Transition & $\lambda, \mathrm{nm}$ & $f$ & Assignment \\
\hline \multirow[t]{3}{*}{$\begin{array}{l}\text { TTC } \\
\left(D_{2 \mathrm{~d}} \text { symmetry }\right. \\
\text { point group })\end{array}$} & $\mathrm{S}_{1}$ & $\mathrm{X}^{1} \mathrm{~A} \rightarrow 1^{1} \mathrm{~A}_{2}$ & 394 & 0 & HOMO-3 $\rightarrow$ LUMO (99\%) \\
\hline & $\mathrm{S}_{2}$ & $\mathrm{X}^{1} \mathrm{~A} \rightarrow 1^{1} \mathrm{~A}_{1}$ & 389 & 0 & HOMO-2 $\rightarrow$ LUMO (98\%) \\
\hline & $\mathrm{S}_{3(4)}$ & $\mathrm{X}^{1} \mathrm{~A} \rightarrow 1^{1} \mathrm{E}$ & 382 & 0.2000 & $\begin{array}{l}\text { HOMO } \rightarrow \text { LUMO }(95 \%) \\
(\text { HOMO-1 } \rightarrow \text { LUMO }(95 \%))\end{array}$ \\
\hline \multicolumn{6}{|c|}{ TTCs (type I) } \\
\hline \multirow{14}{*}{$\begin{array}{l}\mathrm{n}=2 \\
\left(C_{1} \text { symmetry }\right. \\
\text { point group })\end{array}$} & $\mathrm{S}_{1}$ & $\mathrm{X}^{1} \mathrm{~A} \rightarrow 1^{1} \mathrm{~A}$ & 460 & 0.4840 & $\mathrm{HOMO} \rightarrow \mathrm{LUMO}(92 \%)$ \\
\hline & $\mathrm{S}_{2}$ & $\mathrm{X}^{1} \mathrm{~A} \rightarrow 2^{1} \mathrm{~A}$ & 442 & 0.0352 & HOMO-1 $\rightarrow$ LUMO (77\%) \\
\hline & $\mathrm{S}_{3}$ & $\mathrm{X}^{1} \mathrm{~A} \rightarrow 3^{1} \mathrm{~A}$ & 413 & 0.0198 & HOMO-2 $\rightarrow$ LUMO (92\%) \\
\hline & $\mathrm{S}_{4}$ & $\mathrm{X}^{1} \mathrm{~A} \rightarrow 4^{1} \mathrm{~A}$ & 407 & 0.0894 & HOMO-4 $\rightarrow$ LUMO (92\%) \\
\hline & $\mathrm{S}_{5}$ & $\mathrm{X}^{1} \mathrm{~A} \rightarrow 5^{1} \mathrm{~A}$ & 406 & 0.0993 & HOMO-3 $\rightarrow$ LUMO (78\%) \\
\hline & $\mathrm{S}_{8}$ & $\mathrm{X}^{1} \mathrm{~A} \rightarrow 8^{1} \mathrm{~A}$ & 378 & 0.0293 & $\mathrm{HOMO} \rightarrow \mathrm{LUMO}+2(92 \%)$ \\
\hline & $\mathrm{S}_{10}$ & $\mathrm{X}^{1} \mathrm{~A} \rightarrow 10^{1} \mathrm{~A}$ & 373 & 0.0199 & $\begin{array}{l}\text { HOMO- } \rightarrow \text { LUMO+1 }(83 \%) \\
\text { HOMO-7 } \rightarrow \text { LUMO (7\%) } \\
\text { HOMO-3 } \rightarrow \text { LUMO+1 }(5 \%)\end{array}$ \\
\hline & $\mathrm{S}_{11}$ & $\mathrm{X}^{1} \mathrm{~A} \rightarrow 11^{1} \mathrm{~A}$ & 369 & 0.6995 & $\mathrm{HOMO}-1 \rightarrow \mathrm{LUMO}+2(86 \%)$ \\
\hline & $\mathrm{S}_{12}$ & $\mathrm{X}^{1} \mathrm{~A} \rightarrow 12^{1} \mathrm{~A}$ & 346 & 0.0161 & $\begin{array}{l}\text { HOMO-4 } \rightarrow \text { LUMO+2 (88\%) } \\
\text { HOMO-2 } \rightarrow \text { LUMO+1 (8\%) }\end{array}$ \\
\hline & $\mathrm{S}_{15}$ & $\mathrm{X}^{1} \mathrm{~A} \rightarrow 15^{1} \mathrm{~A}$ & 343 & 0.0100 & $\begin{array}{l}\text { HOMO-5 } \rightarrow \text { LUMO+2 }(62 \%) \\
\text { HOMO-7 } \rightarrow \text { LUMO }(14 \%) \\
\text { HOMO-6 } \rightarrow \text { LUMO+2 }(11 \%) \\
\text { HOMO-3 } \rightarrow \text { LUMO+1 }(10 \%)\end{array}$ \\
\hline & $\mathrm{S}_{16}$ & $\mathrm{X}^{1} \mathrm{~A} \rightarrow 16^{1} \mathrm{~A}$ & 342 & 0.1768 & $\mathrm{HOMO}-3 \rightarrow \mathrm{LUMO}+2(93 \%)$ \\
\hline & $\mathrm{S}_{18}$ & $\mathrm{X}^{1} \mathrm{~A} \rightarrow 18^{1} \mathrm{~A}$ & 339 & 0.1444 & $\begin{array}{l}\mathrm{HOMO}-2 \rightarrow \text { LUMO+1 }(85 \%) \\
\mathrm{HOMO}-4 \rightarrow \text { LUMO+2 }(7 \%)\end{array}$ \\
\hline & $\mathrm{S}_{19}$ & $\mathrm{X}^{1} \mathrm{~A} \rightarrow 19^{1} \mathrm{~A}$ & 337 & 0.0217 & HOMO-5 $\rightarrow$ LUMO+1 (84\%) \\
\hline & $\mathrm{S}_{20}$ & $\mathrm{X}^{1} \mathrm{~A} \rightarrow 20^{1} \mathrm{~A}$ & 333 & 0.0385 & $\begin{array}{l}\text { HOMO-3 } \rightarrow \text { LUMO+1 }(50 \%) \\
\text { HOMO-7 } \rightarrow \text { LUMO }(45 \%)\end{array}$ \\
\hline \multirow{10}{*}{$\begin{array}{l}\mathrm{n}=3 \\
\left(C_{\mathrm{s}} \text { symmetry }\right. \\
\text { point group })\end{array}$} & $\mathrm{S}_{1}$ & $\mathrm{X}^{1} \mathrm{~A}^{\prime} \rightarrow 1^{1} \mathrm{~A}^{\prime \prime}$ & 479 & 1.0199 & $\mathrm{HOMO} \rightarrow \mathrm{LUMO}(95 \%)$ \\
\hline & $\mathrm{S}_{2}$ & $\mathrm{X}^{1} \mathrm{~A}^{\prime} \rightarrow 1^{1} \mathrm{~A}^{\prime}$ & 433 & 0.0094 & $\begin{array}{l}\text { HOMO- } \rightarrow \text { LUMO }(60 \%) \\
\text { HOMO } \rightarrow \text { LUMO+1 }(23 \%)\end{array}$ \\
\hline & $\mathrm{S}_{4}$ & $\mathrm{X}^{1} \mathrm{~A}^{\prime} \rightarrow 2^{1} \mathrm{~A}^{\prime}$ & 424 & 0.0967 & $\begin{array}{l}\text { HOMO-2 } \rightarrow \text { LUMO }(81 \%) \\
\text { HOMO-7 } \rightarrow \text { LUMO }(6 \%)\end{array}$ \\
\hline & $\mathrm{S}_{5}$ & $\mathrm{X}^{1} \mathrm{~A}^{\prime} \rightarrow 3^{1} \mathrm{~A}^{\prime}$ & 415 & 0.0023 & $\begin{array}{l}\text { HOMO } \rightarrow \text { LUMO+1 }(67 \%) \\
\text { HOMO-1 } \rightarrow \text { LUMO }(22 \%) \\
\text { HOMO-6 } \rightarrow \text { LUMO }(7 \%)\end{array}$ \\
\hline & $\mathrm{S}_{7}$ & $\mathrm{X}^{1} \mathrm{~A}^{\prime} \rightarrow 3^{1} \mathrm{~A}^{\prime \prime}$ & 407 & 0.0616 & $\begin{array}{l}\text { HOMO-5 } \rightarrow \text { LUMO }(82 \%) \\
\text { HOMO-4 } \rightarrow \text { LUMO+1 }(13 \%)\end{array}$ \\
\hline & $\mathrm{S}_{8}$ & $\mathrm{X}^{1} \mathrm{~A}^{\prime} \rightarrow 5^{1} \mathrm{~A}^{\prime}$ & 404 & 0.0061 & $\begin{array}{l}\text { HOMO-6 } \rightarrow \text { LUMO }(53 \%) \\
\text { HOMO-4 } \rightarrow \text { LUMO }(27 \%) \\
\text { HOMO-5 } \rightarrow \text { LUMO+1 }(9 \%) \\
\text { HOMO-1 } \rightarrow \text { LUMO }(8 \%)\end{array}$ \\
\hline & $\mathrm{S}_{10}$ & $\mathrm{X}^{1} \mathrm{~A}^{\prime} \rightarrow 6^{1} \mathrm{~A}^{\prime}$ & 402 & 0.0586 & $\begin{array}{l}\text { HOMO-7 } \rightarrow \text { LUMO }(74 \%) \\
\text { HOMO-8 } \rightarrow \text { LUMO } 1(14 \%) \\
\text { HOMO-2 } \rightarrow \text { LUMO }(7 \%)\end{array}$ \\
\hline & $\mathrm{S}_{12}$ & $\mathrm{X}^{1} \mathrm{~A}^{\prime} \rightarrow 6^{1} \mathrm{~A}^{\prime \prime}$ & 382 & 0.0774 & $\begin{array}{l}\text { HOMO- } 1 \rightarrow \text { LUMO+1 }(85 \%) \\
\text { HOMO-9 } \rightarrow \text { LUMO }(5 \%)\end{array}$ \\
\hline & $\mathrm{S}_{13}$ & $\mathrm{X}^{1} \mathrm{~A}^{\prime} \rightarrow 7^{1} \mathrm{~A}^{\prime}$ & 381 & 0.0021 & $\begin{array}{l}\mathrm{HOMO} \rightarrow \text { LUMO+3 }(56 \%) \\
\text { HOMO-3 } \rightarrow \text { LUMO }+1(28 \%) \\
\text { HOMO-2 } \rightarrow \text { LUMO }(7 \%)\end{array}$ \\
\hline & $\mathrm{S}_{15}$ & $\mathrm{X}^{1} \mathrm{~A}^{\prime} \rightarrow 8^{1} \mathrm{~A}^{\prime \prime}$ & 378 & 0.2423 & HOMO-9 $\rightarrow$ LUMO (+61\%) \\
\hline
\end{tabular}




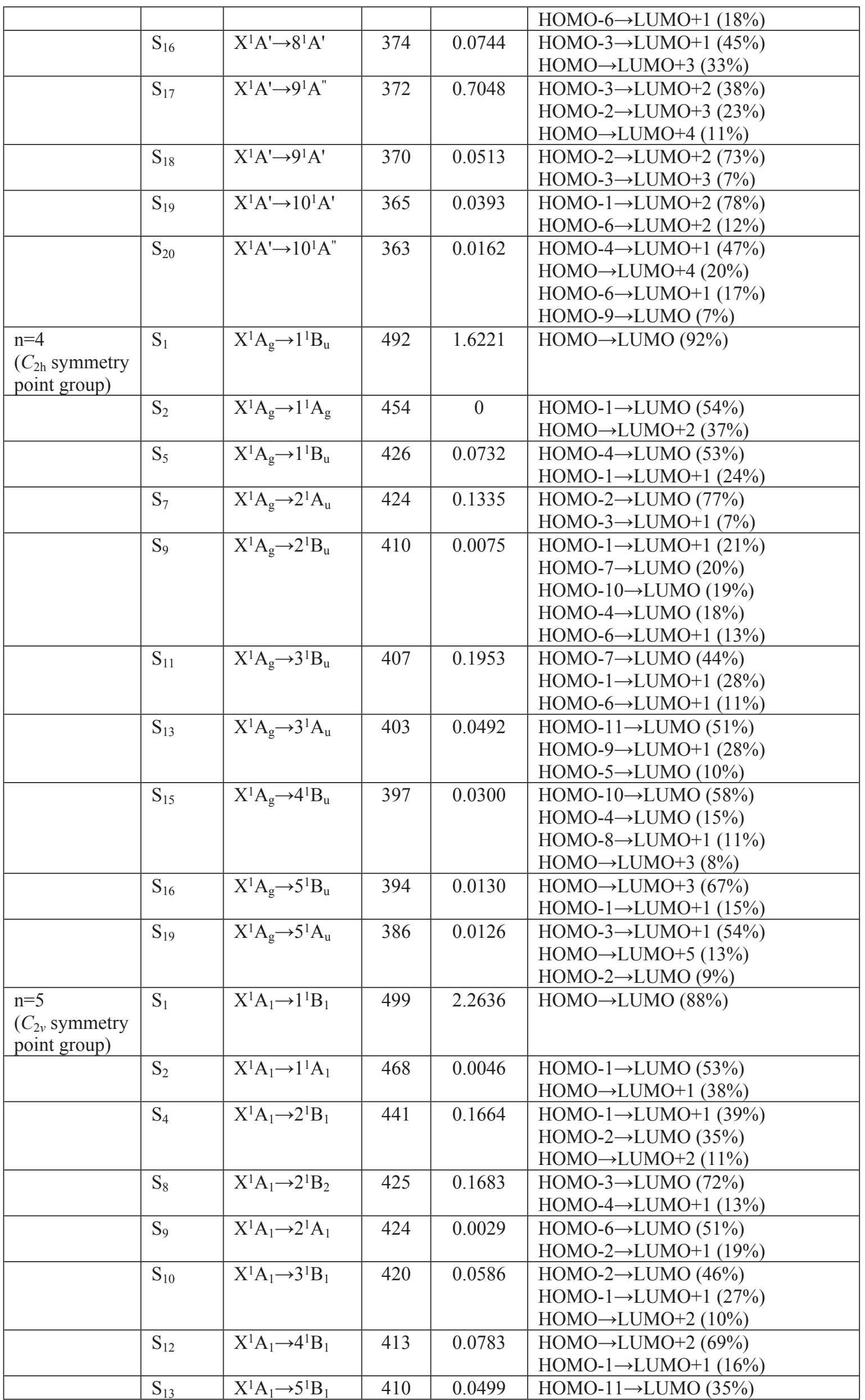




\begin{tabular}{|c|c|c|c|c|c|}
\hline & & & & & $\begin{array}{l}\text { HOMO-8 } \rightarrow \text { LUMO }(28 \%) \\
\text { HOMO-9 } \rightarrow \text { LUMO+1 }(14 \%)\end{array}$ \\
\hline & $\mathrm{S}_{15}$ & $\mathrm{X}^{1} \mathrm{~A}_{1} \rightarrow 6^{1} \mathrm{~B}_{1}$ & 406 & 0.0518 & $\begin{array}{l}\text { HOMO- } 11 \rightarrow \text { LUMO }(31 \%) \\
\text { HOMO-8 } \rightarrow \text { LUMO }(25 \%) \\
\text { HOMO-9 } \rightarrow \text { LUMO+1 }(16 \%) \\
\text { HOMO-10 } \rightarrow \text { LUMO+1 }(12 \%)\end{array}$ \\
\hline & $\mathrm{S}_{18}$ & $\mathrm{X}^{1} \mathrm{~A}_{1} \rightarrow 3^{1} \mathrm{~B}_{2}$ & 403 & 0.0450 & $\begin{array}{l}\text { HOMO- } 12 \rightarrow \text { LUMO }(41 \%) \\
\text { HOMO-13 } \rightarrow \text { LUMO+1 }(32 \%) \\
\text { HOMO-12 } \rightarrow \text { LUMO+2 (9\%) } \\
\text { HOMO-5 } \rightarrow \text { LUMO }(8 \%)\end{array}$ \\
\hline & $\mathrm{S}_{19}$ & $\mathrm{X}^{1} \mathrm{~A}_{1} \rightarrow 5^{1} \mathrm{~A}_{1}$ & 403 & 0.0107 & $\begin{array}{l}\text { HOMO- } \rightarrow \text { LUMO+2 }(25 \%) \\
\text { HOMO-2 } \rightarrow \text { LUMO+1 }(22 \%) \\
\text { HOMO-9 } \rightarrow \text { LUMO }(13 \%) \\
\text { HOMO-6 } \rightarrow \text { LUMO }(10 \%)\end{array}$ \\
\hline \multicolumn{6}{|c|}{ TTCs (type II) } \\
\hline \multirow[t]{10}{*}{$\begin{array}{l}\mathrm{n}=2 \\
\left(C_{2 \mathrm{~h}} \text { symmetry }\right. \\
\text { point group })\end{array}$} & $\mathrm{S}_{1}$ & $\mathrm{X}^{1} \mathrm{~A}_{\mathrm{g}} \rightarrow 1^{1} \mathrm{~A}_{\mathrm{u}}$ & 478 & 0.0013 & $\mathrm{HOMO} \rightarrow \mathrm{LUMO}(97 \%)$ \\
\hline & $\mathrm{S}_{2}$ & $\mathrm{X}^{1} \mathrm{~A}_{\mathrm{g}} \rightarrow 1^{1} \mathrm{~B}_{\mathrm{u}}$ & 460 & 0.3193 & $\begin{array}{l}\text { HOMO } \rightarrow \text { LUMO+1 }(79 \%) \\
\text { HOMO-1 } \rightarrow \text { LUMO }(11 \%)\end{array}$ \\
\hline & $\mathrm{S}_{3}$ & $\mathrm{X}^{1} \mathrm{~A}_{\mathrm{g}} \rightarrow 2^{1} \mathrm{~B}_{\mathrm{u}}$ & 416 & 0.7932 & $\begin{array}{l}\text { HOMO-1 } \rightarrow \text { LUMO }(85 \%) \\
\text { HOMO } \rightarrow \text { LUMO+1 }(11 \%)\end{array}$ \\
\hline & $\mathrm{S}_{6}$ & $\mathrm{X}^{1} \mathrm{~A}_{\mathrm{g}} \rightarrow 2^{1} \mathrm{~A}_{\mathrm{u}}$ & 408 & 0.0053 & HOMO-4 $\rightarrow$ LUMO (93\%) \\
\hline & $\mathrm{S}_{8}$ & $\mathrm{X}^{1} \mathrm{~A}_{\mathrm{g}} \rightarrow 3^{1} \mathrm{~B}_{\mathrm{u}}$ & 396 & 0.6420 & $\begin{array}{l}\text { HOMO-6 } \rightarrow \text { LUMO }(60 \%) \\
\text { HOMO-4 } \rightarrow \text { LUMO+1 }(28 \%)\end{array}$ \\
\hline & $\mathrm{S}_{10}$ & $\mathrm{X}^{1} \mathrm{~A} \rightarrow 3^{1} \mathrm{~A}_{\mathrm{u}}$ & 390 & 0.0235 & $\begin{array}{l}\text { HOMO-6 } \rightarrow \text { LUMO+1 }(65 \%) \\
\text { HOMO-1 } \rightarrow \text { LUMO+1 }(26 \%)\end{array}$ \\
\hline & $\mathrm{S}_{13}$ & $\mathrm{X}^{1} \mathrm{~A}_{\mathrm{g}} \rightarrow 4^{1} \mathrm{~A}_{\mathrm{u}}$ & 383 & 0.2424 & $\begin{array}{l}\text { HOMO-1 } \rightarrow \text { LUMO+1 (64\%) } \\
\text { HOMO-6 } \rightarrow \text { LUMO+1 }(23 \%) \\
\text { HOMO-4 } \rightarrow \text { LUMO }(6 \%)\end{array}$ \\
\hline & $\mathrm{S}_{15}$ & $\mathrm{X}^{1} \mathrm{~A}_{\mathrm{g}} \rightarrow 4^{1} \mathrm{~B}_{\mathrm{u}}$ & 378 & 0.4004 & $\begin{array}{l}\text { HOMO-4 } \rightarrow \text { LUMO+1 }(63 \%) \\
\text { HOMO-6 } \rightarrow \text { LUMO }(30 \%)\end{array}$ \\
\hline & $\mathrm{S}_{18}$ & $\mathrm{X}^{1} \mathrm{~A}_{\mathrm{g}} \rightarrow 5^{1} \mathrm{~B}_{\mathrm{u}}$ & 341 & 0.0161 & HOMO-2 $\rightarrow$ LUMO+2 (91\%) \\
\hline & $\mathrm{S}_{20}$ & $\mathrm{X}^{1} \mathrm{~A}_{\mathrm{g}} \rightarrow 6^{1} \mathrm{~B}_{\mathrm{u}}$ & 336 & 0.0304 & HOMO-5 $\rightarrow$ LUMO+2 (93\%) \\
\hline \multirow{10}{*}{$\begin{array}{l}\mathrm{n}=3 \\
\left(C_{2} \text { symmetry }\right. \\
\text { point group })\end{array}$} & $\mathrm{S}_{1}$ & $\mathrm{X}^{1} \mathrm{~A} \rightarrow 1^{1} \mathrm{~A}$ & 507 & 0 & $\mathrm{HOMO} \rightarrow \mathrm{LUMO}(97 \%)$ \\
\hline & $\mathrm{S}_{2}$ & $\mathrm{X}^{1} \mathrm{~A} \rightarrow 1^{1} \mathrm{~B}$ & 483 & 0.0052 & $\mathrm{HOMO} \rightarrow \mathrm{LUMO}+1(92 \%)$ \\
\hline & $\mathrm{S}_{3}$ & $\mathrm{X}^{1} \mathrm{~A} \rightarrow 2^{1} \mathrm{~B}$ & 479 & 1.0651 & $\mathrm{HOMO} \rightarrow \mathrm{LUMO}+2(89 \%)$ \\
\hline & $\mathrm{S}_{5}$ & $\mathrm{X}^{1} \mathrm{~A} \rightarrow 2^{1} \mathrm{~A}$ & 447 & 0.0008 & $\begin{array}{l}\text { HOMO } \rightarrow \text { LUMO+3 }(36 \%) \\
\text { HOMO-1 } \rightarrow \text { LUMO+2 }(24 \%) \\
\text { HOMO-2 } \rightarrow \text { LUMO }(19 \%) \\
\text { HOMO-9 } \rightarrow \text { LUMO+1 }(6 \%)\end{array}$ \\
\hline & $\mathrm{S}_{7}$ & $\mathrm{X}^{1} \mathrm{~A} \rightarrow 4^{1} \mathrm{~B}$ & 422 & 1.9992 & $\begin{array}{l}\text { HOMO-3 } \rightarrow \text { LUMO (56\%) } \\
\text { HOMO-2 } \rightarrow \text { LUMO+1 }(28 \%)\end{array}$ \\
\hline & $\mathrm{S}_{8}$ & $\mathrm{X}^{1} \mathrm{~A} \rightarrow 4^{1} \mathrm{~A}$ & 417 & 0.0076 & $\begin{array}{l}\text { HOMO } \rightarrow \text { LUMO+3 }(56 \%) \\
\text { HOMO-1 } \rightarrow \text { LUMO }+2(23 \%) \\
\text { HOMO-2 } \rightarrow \text { LUMO }(15 \%)\end{array}$ \\
\hline & $\mathrm{S}_{9}$ & $\mathrm{X}^{1} \mathrm{~A} \rightarrow 5^{1} \mathrm{~A}$ & 412 & 0.0220 & $\begin{array}{l}\text { HOMO-2 } \rightarrow \text { LUMO }(48 \%) \\
\text { HOMO-1 } \rightarrow \text { LUMO+2 }(30 \%) \\
\text { HOMO-6 } \rightarrow \text { LUMO }(6 \%) \\
\text { HOMO-9 } \rightarrow \text { LUMO+1 }(6 \%) \\
\text { HOMO-3 } \rightarrow \text { LUMO+1 }(6 \%)\end{array}$ \\
\hline & $\mathrm{S}_{10}$ & $\mathrm{X}^{1} \mathrm{~A} \rightarrow 5^{1} \mathrm{~B}$ & 411 & 0.0020 & $\begin{array}{l}\text { HOMO-5 } \rightarrow \text { LUMO }(53 \%) \\
\text { HOMO-7 } \rightarrow \text { LUMO+1 }(18 \%) \\
\text { HOMO-8 } \rightarrow \text { LUMO }(17 \%) \\
\text { HOMO-4 } \rightarrow \text { LUMO+1 }(6 \%)\end{array}$ \\
\hline & $\mathrm{S}_{12}$ & $\mathrm{X}^{1} \mathrm{~A} \rightarrow 6^{1} \mathrm{~B}$ & 409 & 0.0126 & $\begin{array}{l}\text { HOMO-8 } \rightarrow \text { LUMO }(73 \%) \\
\text { HOMO-5 } \rightarrow \text { LUMO }(14 \%) \\
\text { HOMO-4 } \rightarrow \text { LUMO+1 }(8 \%)\end{array}$ \\
\hline & $\mathrm{S}_{14}$ & $\mathrm{X}^{1} \mathrm{~A} \rightarrow 7^{1} \mathrm{~B}$ & 403 & 0.6169 & HOMO-9 $\rightarrow$ LUMO (43\%) \\
\hline
\end{tabular}




\begin{tabular}{|c|c|c|c|c|c|}
\hline & & & & & $\begin{array}{l}\text { HOMO-6 } \rightarrow \text { LUMO+1 (27\%) } \\
\text { HOMO-3 } \rightarrow \text { LUMO }(16 \%)\end{array}$ \\
\hline & $\mathrm{S}_{15}$ & $\mathrm{X}^{1} \mathrm{~A} \rightarrow 8^{1} \mathrm{~A}$ & 400 & 0.0184 & $\begin{array}{l}\text { HOMO-6 } \rightarrow \text { LUMO }(59 \%) \\
\text { HOMO-3 } \rightarrow \text { LUMO+1 }(18 \%) \\
\text { HOMO-1 } \rightarrow \text { LUMO+2 }(8 \%)\end{array}$ \\
\hline & $\mathrm{S}_{16}$ & $\mathrm{X}^{1} \mathrm{~A} \rightarrow 8^{1} \mathrm{~B}$ & 394 & 0.0801 & $\begin{array}{l}\text { HOMO-2 } \rightarrow \text { LUMO+1 }(47 \%) \\
\text { HOMO-9 } \rightarrow \text { LUMO }(20 \%) \\
\text { HOMO-3 } \rightarrow \text { LUMO }(10 \%) \\
\text { HOMO-1 } \rightarrow \text { LUMO+3 }(9 \%)\end{array}$ \\
\hline & $\mathrm{S}_{17}$ & $\mathrm{X}^{1} \mathrm{~A} \rightarrow 9^{1} \mathrm{~B}$ & 392 & 0.2060 & $\begin{array}{l}\text { HOMO-1 } \rightarrow \text { LUMO+3 (54\%) } \\
\text { HOMO } \rightarrow \text { LUMO+4 (10\%) } \\
\text { HOMO-2 } \rightarrow \text { LUMO+1 (9\%) } \\
\text { HOMO-4 } \rightarrow \text { LUMO+3 (9\%) }\end{array}$ \\
\hline & $\mathrm{S}_{19}$ & $\mathrm{X}^{1} \mathrm{~A} \rightarrow 10^{1} \mathrm{~A}$ & 388 & 0.0122 & $\begin{array}{l}\text { HOMO-5 } \rightarrow \text { LUMO+2 }(27 \%) \\
\text { HOMO-3 } \rightarrow \text { LUMO+1 }(22 \%) \\
\text { HOMO-8 } \rightarrow \text { LUMO+2 }(12 \%) \\
\text { HOMO-9 } \rightarrow \text { LUMO+1 }(11 \%)\end{array}$ \\
\hline \multirow[t]{11}{*}{$\begin{array}{l}\mathrm{n}=4 \\
\left(C_{1} \text { symmetry }\right. \\
\text { point group })\end{array}$} & $\mathrm{S}_{1}$ & $\mathrm{X}^{1} \mathrm{~A} \rightarrow 1^{1} \mathrm{~A}$ & 518 & 0 & $\mathrm{HOMO} \rightarrow \mathrm{LUMO}(94 \%)$ \\
\hline & $\mathrm{S}_{2}$ & $\mathrm{X}^{1} \mathrm{~A} \rightarrow 2^{1} \mathrm{~A}$ & 498 & 0.0019 & $\begin{array}{l}\mathrm{HOMO} \rightarrow \text { LUMO+1 }(76 \%) \\
\text { HOMO-1 } \rightarrow \text { LUMO }(19 \%)\end{array}$ \\
\hline & $\mathrm{S}_{3}$ & $\mathrm{X}^{1} \mathrm{~A} \rightarrow 3^{1} \mathrm{~A}$ & 489 & 1.9529 & $\mathrm{HOMO} \rightarrow \mathrm{LUMO}+2(86 \%)$ \\
\hline & $\mathrm{S}_{4}$ & $\mathrm{X}^{1} \mathrm{~A} \rightarrow 4^{1} \mathrm{~A}$ & 484 & 0.0094 & $\begin{array}{l}\text { HOMO } \rightarrow \text { LUMO+3 }(77 \%) \\
\text { HOMO-1 } \rightarrow \text { LUMO+1 (18\%) }\end{array}$ \\
\hline & $\mathrm{S}_{9}$ & $\mathrm{X}^{1} \mathrm{~A} \rightarrow 9^{1} \mathrm{~A}$ & 441 & 0.0241 & $\begin{array}{l}\text { HOMO- } \rightarrow \text { LUMO+4 }(22 \%) \\
\text { HOMO-3 } \rightarrow \text { LUMO }(20 \%) \\
\text { HOMO-2 } \rightarrow \text { LUMO+2 }(13 \%) \\
\text { HOMO } \rightarrow \text { LUMO+5 }(12 \%)\end{array}$ \\
\hline & $\mathrm{S}_{12}$ & $\mathrm{X}^{1} \mathrm{~A} \rightarrow 12^{1} \mathrm{~A}$ & 425 & 3.2847 & $\begin{array}{l}\mathrm{HOMO}-6 \rightarrow \text { LUMO }(30 \%) \\
\mathrm{HOMO}-4 \rightarrow \mathrm{LUMO}+1(23 \%) \\
\mathrm{HOMO}-3 \rightarrow \text { LUMO+3 }(15 \%)\end{array}$ \\
\hline & $\mathrm{S}_{15}$ & $\mathrm{X}^{1} \mathrm{~A} \rightarrow 15^{1} \mathrm{~A}$ & 413 & 0.0065 & $\begin{array}{l}\text { HOMO- } 10 \rightarrow \text { LUMO }(46 \%) \\
\text { HOMO-2 } \rightarrow \text { LUMO+3 }(24 \%) \\
\text { HOMO-8 } \rightarrow \text { LUMO+1 }(13 \%)\end{array}$ \\
\hline & $\mathrm{S}_{16}$ & $\mathrm{X}^{1} \mathrm{~A} \rightarrow 16^{1} \mathrm{~A}$ & 411 & 0.0930 & $\begin{array}{l}\mathrm{HOMO}-3 \rightarrow \text { LUMO }(38 \%) \\
\mathrm{HOMO}-1 \rightarrow \text { LUMO+4 }(34 \%) \\
\mathrm{HOMO}-4 \rightarrow \text { LUMO+1 }(6 \%)\end{array}$ \\
\hline & $\mathrm{S}_{17}$ & $\mathrm{X}^{1} \mathrm{~A} \rightarrow 17^{1} \mathrm{~A}$ & 410.6 & 0.0012 & $\begin{array}{l}\text { HOMO-7 } \rightarrow \text { LUMO+1 }(31 \%) \\
\text { HOMO-5 } \rightarrow \text { LUMO }(20 \%) \\
\text { HOMO-8 } \rightarrow \text { LUMO }(16 \%) \\
\text { HOMO-12 } \rightarrow \text { LUMO }(6 \%) \\
\text { HOMO-8 } \rightarrow \text { LUMO+3 }(6 \%)\end{array}$ \\
\hline & $\mathrm{S}_{18}$ & $\mathrm{X}^{1} \mathrm{~A} \rightarrow 18^{1} \mathrm{~A}$ & 410.4 & 0.0104 & $\begin{array}{l}\text { HOMO-7 } \rightarrow \text { LUMO }(42 \%) \\
\text { HOMO-5 } \rightarrow \text { LUMO+1 }(24 \%) \\
\text { HOMO-8 } \rightarrow \text { LUMO+1 }(16 \%) \\
\text { HOMO-7 } \rightarrow \text { LUMO+3 }(10 \%)\end{array}$ \\
\hline & $\mathrm{S}_{20}$ & $\mathrm{X}^{1} \mathrm{~A}_{1} \rightarrow 20^{1} \mathrm{~A}$ & 406 & 0.4042 & $\begin{array}{l}\mathrm{HOMO} \rightarrow \mathrm{LUMO}+5(75 \%) \\
\mathrm{HOMO}-2 \rightarrow \mathrm{LUMO}+2(8 \%)\end{array}$ \\
\hline \multirow[t]{6}{*}{$\begin{array}{l}\mathrm{n}=5 \\
\left(C_{2 v} \text { symmetry }\right. \\
\text { point group })\end{array}$} & $\mathrm{S}_{1}$ & $\mathrm{X}^{1} \mathrm{~A}_{1} \rightarrow 1^{1} \mathrm{~A}_{2}$ & 523 & 0 & $\mathrm{HOMO} \rightarrow \mathrm{LUMO}(90 \%)$ \\
\hline & $\mathrm{S}_{2}$ & $\mathrm{X}^{1} \mathrm{~A}_{1} \rightarrow 1^{1} \mathrm{~B}_{2}$ & 509 & 0.0001 & $\begin{array}{l}\text { HOMO } \rightarrow \text { LUMO+1 }(65 \%) \\
\text { HOMO-1 } \rightarrow \text { LUMO }(28 \%)\end{array}$ \\
\hline & $\mathrm{S}_{3}$ & $\mathrm{X}^{1} \mathrm{~A}_{1} \rightarrow 1^{1} \mathrm{~B}_{1}$ & 495 & 3.0042 & $\begin{array}{l}\mathrm{HOMO} \rightarrow \mathrm{LUMO}+3(81 \%) \\
\mathrm{HOMO}-1 \rightarrow \mathrm{LUMO}+5(11 \%)\end{array}$ \\
\hline & $\mathrm{S}_{5}$ & $\mathrm{X}^{1} \mathrm{~A}_{1} \rightarrow 2^{1} \mathrm{~B}_{2}$ & 484 & 0.0131 & $\begin{array}{l}\mathrm{HOMO} \rightarrow \mathrm{LUMO}+4(61 \%) \\
\mathrm{HOMO}-1 \rightarrow \mathrm{LUMO}+2(29 \%)\end{array}$ \\
\hline & $\mathrm{S}_{7}$ & $\mathrm{X}^{1} \mathrm{~A}_{1} \rightarrow 1^{1} \mathrm{~A}_{1}$ & 472 & 0.0032 & $\begin{array}{l}\mathrm{HOMO}-1 \rightarrow \mathrm{LUMO}+3(42 \%) \\
\mathrm{HOMO} \rightarrow \mathrm{LUMO}+5(39 \%)\end{array}$ \\
\hline & $\mathrm{S}_{9}$ & $\mathrm{X}^{1} \mathrm{~A}_{1} \rightarrow 2^{1} \mathrm{~B}_{1}$ & 453 & 0.0878 & HOMO-1 $\rightarrow$ LUMO+5 (28\%) \\
\hline
\end{tabular}




\begin{tabular}{|c|c|c|c|c|c|}
\hline & & & & & $\begin{array}{l}\mathrm{HOMO}-2 \rightarrow \mathrm{LUMO}+3(20 \%) \\
\mathrm{HOMO} \rightarrow \mathrm{LUMO}+6(18 \%)\end{array}$ \\
\hline & $\mathrm{S}_{15}$ & $\mathrm{X}^{1} \mathrm{~A}_{1} \rightarrow 3^{1} \mathrm{~A}_{1}$ & 438 & 0.0015 & $\begin{array}{l}\text { HOMO-2 } \rightarrow \text { LUMO+5 }(15 \%) \\
\text { HOMO-4 } \rightarrow \text { LUMO }(14 \%) \\
\text { HOMO } \rightarrow \text { LUMO+5 }(12 \%) \\
\text { HOMO-6 } \rightarrow \text { LUMO }(10 \%) \\
\text { HOMO-3 } \rightarrow \text { LUMO+3 }(7 \%) \\
\text { HOMO-5 } \rightarrow \text { LUMO+1 }(7 \%)\end{array}$ \\
\hline & $\mathrm{S}_{16}$ & $\mathrm{X}^{1} \mathrm{~A}_{1} \rightarrow 3^{1} \mathrm{~B}_{1}$ & 427 & 3.8679 & $\begin{array}{l}\text { HOMO-9 } \rightarrow \text { LUMO }(21 \%) \\
\text { HOMO-6 } \rightarrow \text { LUMO+1 }(16 \%) \\
\text { HOMO-13 } \rightarrow \text { LUMO }(16 \%) \\
\text { HOMO-5 } \rightarrow \text { LUMO+2 }(11 \%)\end{array}$ \\
\hline & $\mathrm{S}_{19}$ & $\mathrm{X}^{1} \mathrm{~A}_{1} \rightarrow 4^{1} \mathrm{~B}_{1}$ & 421 & 1.2655 & $\begin{array}{l}\mathrm{HOMO} \rightarrow \mathrm{LUMO}+6(52 \%) \\
\mathrm{HOMO}-1 \rightarrow \mathrm{LUMO}+5(19 \%) \\
\mathrm{HOMO} \rightarrow \mathrm{LUMO}+3(8 \%) \\
\text { HOMO-2 } \rightarrow \text { LUMO+3 (7\%) }\end{array}$ \\
\hline & $\mathrm{S}_{20}$ & $\mathrm{X}^{1} \mathrm{~A}_{1} \rightarrow 7^{1} \mathrm{~B}_{2}$ & 420 & 0.0035 & $\begin{array}{l}\text { HOMO-3 } \rightarrow \text { LUMO }(50 \%) \\
\text { HOMO-2 } \rightarrow \text { LUMO+4 }(11 \%) \\
\text { HOMO-3 } \rightarrow \text { LUMO+2 }(9 \%) \\
\text { HOMO-2 } \rightarrow \text { LUMO+1 }(8 \%) \\
\text { HOMO- } 12 \rightarrow \text { LUMO }(7 \%)\end{array}$ \\
\hline \multicolumn{6}{|c|}{ TTCs (type III) } \\
\hline \multirow{14}{*}{$\begin{array}{l}\mathrm{n}=2 \\
\left(D_{2} \text { symmetry }\right. \\
\text { point group) }\end{array}$} & $\mathrm{S}_{1}$ & $\mathrm{X}^{1} \mathrm{~A} \rightarrow 1^{1} \mathrm{~B}_{1}$ & 639 & 0.0012 & $\mathrm{HOMO} \rightarrow \mathrm{LUMO}(98 \%)$ \\
\hline & $\mathrm{S}_{2}$ & $\mathrm{X}^{1} \mathrm{~A} \rightarrow 1^{1} \mathrm{~B}_{2}$ & 480 & 0.0427 & $\mathrm{HOMO} \rightarrow \mathrm{LUMO}+1(89 \%)$ \\
\hline & $\mathrm{S}_{3}$ & $\mathrm{X}^{1} \mathrm{~A} \rightarrow 1{ }^{1} \mathrm{~B}_{3}$ & 470 & 0.3011 & $\begin{array}{l}\text { HOMO- } \rightarrow \text { LUMO }(79 \%) \\
\text { HOMO-2 } \rightarrow \text { LUMO }(15 \%)\end{array}$ \\
\hline & $\mathrm{S}_{4}$ & $\mathrm{X}^{1} \mathrm{~A} \rightarrow 2^{1} \mathrm{~B}_{1}$ & 450 & 0.0011 & HOMO-5 $\rightarrow$ LUMO (95\%) \\
\hline & $\mathrm{S}_{5}$ & $\mathrm{X}^{1} \mathrm{~A} \rightarrow 2^{1} \mathrm{~B}_{3}$ & 447 & 0.2182 & $\begin{array}{l}\text { HOMO-2 } \rightarrow \text { LUMO }(81 \%) \\
\text { HOMO-1 } \rightarrow \text { LUMO }(15 \%)\end{array}$ \\
\hline & $\mathrm{S}_{7}$ & $\mathrm{X}^{1} \mathrm{~A} \rightarrow 2^{1} \mathrm{~B}_{2}$ & 444 & 0.0918 & $\begin{array}{l}\text { HOMO-4 } \rightarrow \text { LUMO }(82 \%) \\
\text { HOMO } \rightarrow \text { LUMO+1 }(9 \%)\end{array}$ \\
\hline & $\mathrm{S}_{9}$ & $\mathrm{X}^{1} \mathrm{~A} \rightarrow 3^{1} \mathrm{~B}_{2}$ & 406 & 0.0586 & $\begin{array}{l}\text { HOMO-7 } \rightarrow \text { LUMO }(85 \%) \\
\text { HOMO-4 } \rightarrow \text { LUMO }(7 \%)\end{array}$ \\
\hline & $\mathrm{S}_{11}$ & $\mathrm{X}^{1} \mathrm{~A} \rightarrow 3^{1} \mathrm{~B}_{3}$ & 367 & 0.6961 & $\begin{array}{l}\mathrm{HOMO} \rightarrow \mathrm{LUMO}+2(47 \%) \\
\mathrm{HOMO}-3 \rightarrow \mathrm{LUMO}+1(45 \%)\end{array}$ \\
\hline & $\mathrm{S}_{14}$ & $\mathrm{X}^{1} \mathrm{~A} \rightarrow 4^{1} \mathrm{~B}_{3}$ & 359 & 0.4338 & $\begin{array}{l}\mathrm{HOMO}-3 \rightarrow \mathrm{LUMO}+1(47 \%) \\
\mathrm{HOMO} \rightarrow \mathrm{LUMO}+2(40 \%)\end{array}$ \\
\hline & $\mathrm{S}_{15}$ & $\mathrm{X}^{1} \mathrm{~A} \rightarrow 4^{1} \mathrm{~B}_{1}$ & 358.7 & 0.0023 & $\mathrm{HOMO}-4 \rightarrow \mathrm{LUMO}+1(88 \%)$ \\
\hline & $\mathrm{S}_{16}$ & $\mathrm{X}^{1} \mathrm{~A} \rightarrow 4^{1} \mathrm{~B}_{2}$ & 358.3 & 0.1153 & $\begin{array}{l}\text { HOMO-5 } \rightarrow \text { LUMO+1 }(87 \%) \\
\text { HOMO-7 } \rightarrow \text { LUMO }(6 \%)\end{array}$ \\
\hline & $\mathrm{S}_{17}$ & $\mathrm{X}^{1} \mathrm{~A} \rightarrow 5^{1} \mathrm{~B}_{3}$ & 346 & 0.0219 & HOMO-6 $\rightarrow$ LUMO+1 (91\%) \\
\hline & $\mathrm{S}_{18}$ & $\mathrm{X}^{1} \mathrm{~A} \rightarrow 5^{1} \mathrm{~B}_{1}$ & 337 & 0.0015 & $\begin{array}{l}\mathrm{HOMO}-7 \rightarrow \mathrm{LUMO}+1(82 \%) \\
\mathrm{HOMO} \rightarrow \mathrm{LUMO}+3(9 \%)\end{array}$ \\
\hline & $\mathrm{S}_{19}$ & $\mathrm{X}^{1} \mathrm{~A} \rightarrow 6^{1} \mathrm{~B}_{3}$ & 317 & 0.2646 & $\begin{array}{l}\text { HOMO-8 } \rightarrow \text { LUMO (71\%) } \\
\text { HOMO } \rightarrow \text { LUMO+6 (17\%) }\end{array}$ \\
\hline \multirow[t]{8}{*}{$\begin{array}{l}\mathrm{n}=3 \\
\left(D_{2} \text { symmetry }\right. \\
\text { point group) }\end{array}$} & $\mathrm{S}_{1}$ & $\mathrm{X}^{1} \mathrm{~A} \rightarrow 1^{1} \mathrm{~B}_{1}$ & 700 & 0.0001 & HOMO $\rightarrow$ LUMO (95\%) \\
\hline & $\mathrm{S}_{2}$ & $\mathrm{X}^{1} \mathrm{~A} \rightarrow 1^{1} \mathrm{~B}_{2}$ & 660 & 0.0008 & HOMO-1 $\rightarrow$ LUMO (95\%) \\
\hline & $\mathrm{S}_{3}$ & $\mathrm{X}^{1} \mathrm{~A} \rightarrow 2^{1} \mathrm{~B}_{2}$ & 543 & 0.0382 & $\mathrm{HOMO} \rightarrow \mathrm{LUMO}+1(91 \%)$ \\
\hline & $\mathrm{S}_{5}$ & $\mathrm{X}^{1} \mathrm{~A} \rightarrow 1^{1} \mathrm{~B}_{3}$ & 495 & 0.8767 & HOMO-2 $\rightarrow$ LUMO (89\%) \\
\hline & $\mathrm{S}_{8}$ & $\mathrm{X}^{1} \mathrm{~A} \rightarrow 2^{1} \mathrm{~B}_{3}$ & 462 & 0.1297 & $\begin{array}{l}\text { HOMO-5 } \rightarrow \text { LUMO }(84 \%) \\
\text { HOMO-4 } \rightarrow \text { LUMO+1 (8\%) }\end{array}$ \\
\hline & $\mathrm{S}_{10}$ & $\mathrm{X}^{1} \mathrm{~A} \rightarrow 3^{1} \mathrm{~B}_{2}$ & 461 & 0.0101 & $\begin{array}{l}\text { HOMO-7 } \rightarrow \text { LUMO }(74 \%) \\
\text { HOMO-1 } \rightarrow \text { LUMO+2 }(11 \%) \\
\text { HOMO-6 } \rightarrow \text { LUMO+1 }(11 \%)\end{array}$ \\
\hline & $\mathrm{S}_{11}$ & $\mathrm{X}^{1} \mathrm{~A} \rightarrow 4^{1} \mathrm{~B}_{1}$ & 458 & 0.0011 & $\begin{array}{l}\text { HOMO-6 } \rightarrow \text { LUMO }(54 \%) \\
\text { HOMO } \rightarrow \text { LUMO+2 }(38 \%)\end{array}$ \\
\hline & $\mathrm{S}_{13}$ & $\mathrm{X}^{1} \mathrm{~A} \rightarrow 4^{1} \mathrm{~B}_{2}$ & 449 & 0.0894 & $\mathrm{HOMO}-1 \rightarrow \mathrm{LUMO}+2(81 \%)$ \\
\hline
\end{tabular}




\begin{tabular}{|c|c|c|c|c|c|}
\hline & & & & & HOMO-7 $\rightarrow$ LUMO (10\%) \\
\hline & $\mathrm{S}_{16}$ & $\mathrm{X}^{1} \mathrm{~A} \rightarrow 5^{1} \mathrm{~B}_{2}$ & 421 & 0.0718 & $\begin{array}{l}\text { HOMO- } 10 \rightarrow \text { LUMO }(81 \%) \\
\text { HOMO-6 } \rightarrow \text { LUMO+ } 1(5 \%)\end{array}$ \\
\hline & $\mathrm{S}_{20}$ & $\mathrm{X}^{1} \mathrm{~A} \rightarrow 5^{1} \mathrm{~B}_{3}$ & 400 & 0.1106 & $\begin{array}{l}\text { HOMO-4 } \rightarrow \text { LUMO+1 (69\%) } \\
\text { HOMO-3 } \rightarrow \text { LUMO+1 }(11 \%) \\
\text { HOMO-5 } \rightarrow \text { LUMO }(7 \%)\end{array}$ \\
\hline \multirow{15}{*}{$\begin{array}{l}\mathrm{n}=4 \\
\left(D_{2} \text { symmetry }\right. \\
\text { point group) }\end{array}$} & $\mathrm{S}_{1}$ & $\mathrm{X}^{1} \mathrm{~A} \rightarrow 1^{1} \mathrm{~B}_{1}$ & 723 & 0.0008 & $\mathrm{HOMO} \rightarrow \mathrm{LUMO}(50 \%)$ \\
\hline & $\mathrm{S}_{2}$ & $\mathrm{X}^{1} \mathrm{~A} \rightarrow 1^{1} \mathrm{~B}_{2}$ & 689 & 0.0006 & $\begin{array}{l}\text { HOMO- } \rightarrow \text { LUMO }(80 \%) \\
\text { HOMO } \rightarrow \text { LUMO+1 }(18 \%)\end{array}$ \\
\hline & $\mathrm{S}_{3}$ & $\mathrm{X}^{1} \mathrm{~A} \rightarrow 2^{1} \mathrm{~B}_{1}$ & 670 & 0.0007 & $\begin{array}{l}\text { HOMO-2 } \rightarrow \text { LUMO (56\%) } \\
\text { HOMO } \rightarrow \text { LUMO }(34 \%) \\
\text { HOMO-1 } \rightarrow \text { LUMO+1 }(9 \%)\end{array}$ \\
\hline & $\mathrm{S}_{4}$ & $\mathrm{X}^{1} \mathrm{~A} \rightarrow 2^{1} \mathrm{~B}_{2}$ & 587 & 0.0229 & $\begin{array}{l}\text { HOMO-2 } \rightarrow \text { LUMO+1 }(65 \%) \\
\text { HOMO } \rightarrow \text { LUMO+1 }(24 \%) \\
\text { HOMO-1 } \rightarrow \text { LUMO }(8 \%)\end{array}$ \\
\hline & $\mathrm{S}_{5}$ & $\mathrm{X}^{1} \mathrm{~A} \rightarrow 3^{1} \mathrm{~B}_{1}$ & 577 & 0.0013 & $\begin{array}{l}\mathrm{HOMO}-1 \rightarrow \text { LUMO+1 }(74 \%) \\
\mathrm{HOMO} \rightarrow \text { LUMO }(15 \%) \\
\mathrm{HOMO} \rightarrow \text { LUMO }+2(9 \%)\end{array}$ \\
\hline & $\mathrm{S}_{6}$ & $\mathrm{X}^{1} \mathrm{~A} \rightarrow 3^{1} \mathrm{~B}_{2}$ & 576 & 0.0016 & $\begin{array}{l}\mathrm{HOMO} \rightarrow \text { LUMO+1 }(49 \%) \\
\mathrm{HOMO}-2 \rightarrow \text { LUMO+1 }(30 \%) \\
\text { HOMO-1 } \rightarrow \text { LUMO }(11 \%)\end{array}$ \\
\hline & $\mathrm{S}_{7}$ & $\mathrm{X}^{1} \mathrm{~A} \rightarrow 1^{1} \mathrm{~B}_{3}$ & 506 & 1.4637 & $\begin{array}{l}\text { HOMO-3 } \rightarrow \text { LUMO }(81 \%) \\
\text { HOMO-7 } \rightarrow \text { LUMO }(7 \%) \\
\text { HOMO-4 } \rightarrow \text { LUMO+1 (7\%) }\end{array}$ \\
\hline & $\mathrm{S}_{8}$ & $\mathrm{X}^{1} \mathrm{~A} \rightarrow 4^{1} \mathrm{~B}_{1}$ & 506 & 0.0042 & $\begin{array}{l}\mathrm{HOMO} \rightarrow \mathrm{LUMO}+2(73 \%) \\
\mathrm{HOMO}-1 \rightarrow \mathrm{LUMO}+3(8 \%) \\
\mathrm{HOMO}-2 \rightarrow \mathrm{LUMO}+2(7 \%) \\
\mathrm{HOMO}-1 \rightarrow \mathrm{LUMO}+1(6 \%)\end{array}$ \\
\hline & $\mathrm{S}_{9}$ & $\mathrm{X}^{1} \mathrm{~A} \rightarrow 4^{1} \mathrm{~B}_{2}$ & 504 & 0.0471 & $\begin{array}{l}\mathrm{HOMO}-1 \rightarrow \mathrm{LUMO}+2(76 \%) \\
\mathrm{HOMO} \rightarrow \mathrm{LUMO}+3(11 \%) \\
\mathrm{HOMO} \rightarrow \mathrm{LUMO}+1(7 \%)\end{array}$ \\
\hline & $\mathrm{S}_{10}$ & $\mathrm{X}^{1} \mathrm{~A} \rightarrow 5^{1} \mathrm{~B}_{1}$ & 497 & 0.0023 & $\mathrm{HOMO}-2 \rightarrow \mathrm{LUMO}+2(87 \%)$ \\
\hline & $\mathrm{S}_{11}$ & $\mathrm{X}^{1} \mathrm{~A} \rightarrow 2^{1} \mathrm{~B}_{3}$ & 490 & 0.0229 & $\begin{array}{l}\text { HOMO-7 } \rightarrow \text { LUMO }(59 \%) \\
\text { HOMO-5 } \rightarrow \text { LUMO }(25 \%) \\
\text { HOMO-4 } \rightarrow \text { LUMO+1 }(5 \%)\end{array}$ \\
\hline & $\mathrm{S}_{14}$ & $\mathrm{X}^{1} \mathrm{~A} \rightarrow 3^{1} \mathrm{~B}_{3}$ & 465 & 0.0877 & $\begin{array}{l}\text { HOMO-5 } \rightarrow \text { LUMO }(50 \%) \\
\text { HOMO-7 } \rightarrow \text { LUMO }(19 \%) \\
\text { HOMO-6 } \rightarrow \text { LUMO+1 }(16 \%) \\
\text { HOMO-11 } \rightarrow \text { LUMO }(7 \%)\end{array}$ \\
\hline & $\mathrm{S}_{16}$ & $\mathrm{X}^{1} \mathrm{~A} \rightarrow 6^{1} \mathrm{~B}_{1}$ & 463.8 & 0.0040 & $\begin{array}{l}\text { HOMO-8 } \rightarrow \text { LUMO }(71 \%) \\
\text { HOMO-9 } \rightarrow \text { LUMO+1 }(21 \%)\end{array}$ \\
\hline & $\mathrm{S}_{17}$ & $\mathrm{X}^{1} \mathrm{~A} \rightarrow 5^{1} \mathrm{~B}_{2}$ & 463.8 & 0.0285 & $\begin{array}{l}\text { HOMO-9 } \rightarrow \text { LUMO }(71 \%) \\
\text { HOMO-8 } \rightarrow \text { LUMO+1 }(21 \%)\end{array}$ \\
\hline & $\mathrm{S}_{20}$ & $\mathrm{X}^{1} \mathrm{~A} \rightarrow 7^{1} \mathrm{~B}_{1}$ & 441 & 0.0059 & $\begin{array}{l}\mathrm{HOMO}-1 \rightarrow \mathrm{LUMO}+3(84 \%) \\
\mathrm{HOMO} \rightarrow \mathrm{LUMO}+2(9 \%)\end{array}$ \\
\hline \multirow[t]{5}{*}{$\begin{array}{l}\mathrm{n}=5 \\
\left(D_{2} \text { symmetry }\right. \\
\text { point group) }\end{array}$} & $\mathrm{S}_{1}$ & $\mathrm{X}^{1} \mathrm{~A} \rightarrow 1^{1} \mathrm{~B}_{1}$ & 730 & 0 & $\begin{array}{l}\text { HOMO-2 } \rightarrow \text { LUMO }(65 \%) \\
\text { HOMO } \rightarrow \text { LUMO }(18 \%) \\
\text { HOMO-3 } \rightarrow \text { LUMO+1 }(6 \%) \\
\text { HOMO-1 } \rightarrow \text { LUMO+1 }(5 \%)\end{array}$ \\
\hline & $\mathrm{S}_{2}$ & $\mathrm{X}^{1} \mathrm{~A} \rightarrow 1^{1} \mathrm{~B}_{2}$ & 707 & 0.0002 & $\begin{array}{l}\text { HOMO-3 } \rightarrow \text { LUMO }(43 \%) \\
\text { HOMO-1 } \rightarrow \text { LUMO }(27 \%) \\
\text { HOMO } \rightarrow \text { LUMO+1 }(20 \%)\end{array}$ \\
\hline & $\mathrm{S}_{3}$ & $\mathrm{X}^{1} \mathrm{~A} \rightarrow 3^{1} \mathrm{~B}_{1}$ & 683 & 0.0001 & $\begin{array}{l}\mathrm{HOMO} \rightarrow \text { LUMO }(45 \%) \\
\text { HOMO- } \rightarrow \text { LUMO+1 }(25 \%) \\
\text { HOMO-2 } \rightarrow \text { LUMO }(24 \%)\end{array}$ \\
\hline & $\mathrm{S}_{4}$ & $\mathrm{X}^{1} \mathrm{~A} \rightarrow 2^{1} \mathrm{~B}_{2}$ & 674 & 0.0003 & $\begin{array}{l}\text { HOMO-3 } \rightarrow \text { LUMO }(45 \%) \\
\text { HOMO- } \rightarrow \text { LUMO }(36 \%) \\
\text { HOMO } \rightarrow \text { LUMO+1 }(12 \%)\end{array}$ \\
\hline & $\mathrm{S}_{6}$ & $\mathrm{X}^{1} \mathrm{~A} \rightarrow 3^{1} \mathrm{~B}_{2}$ & 602 & 0.0251 & HOMO-2 $\rightarrow$ LUMO+1 (63\%) \\
\hline
\end{tabular}




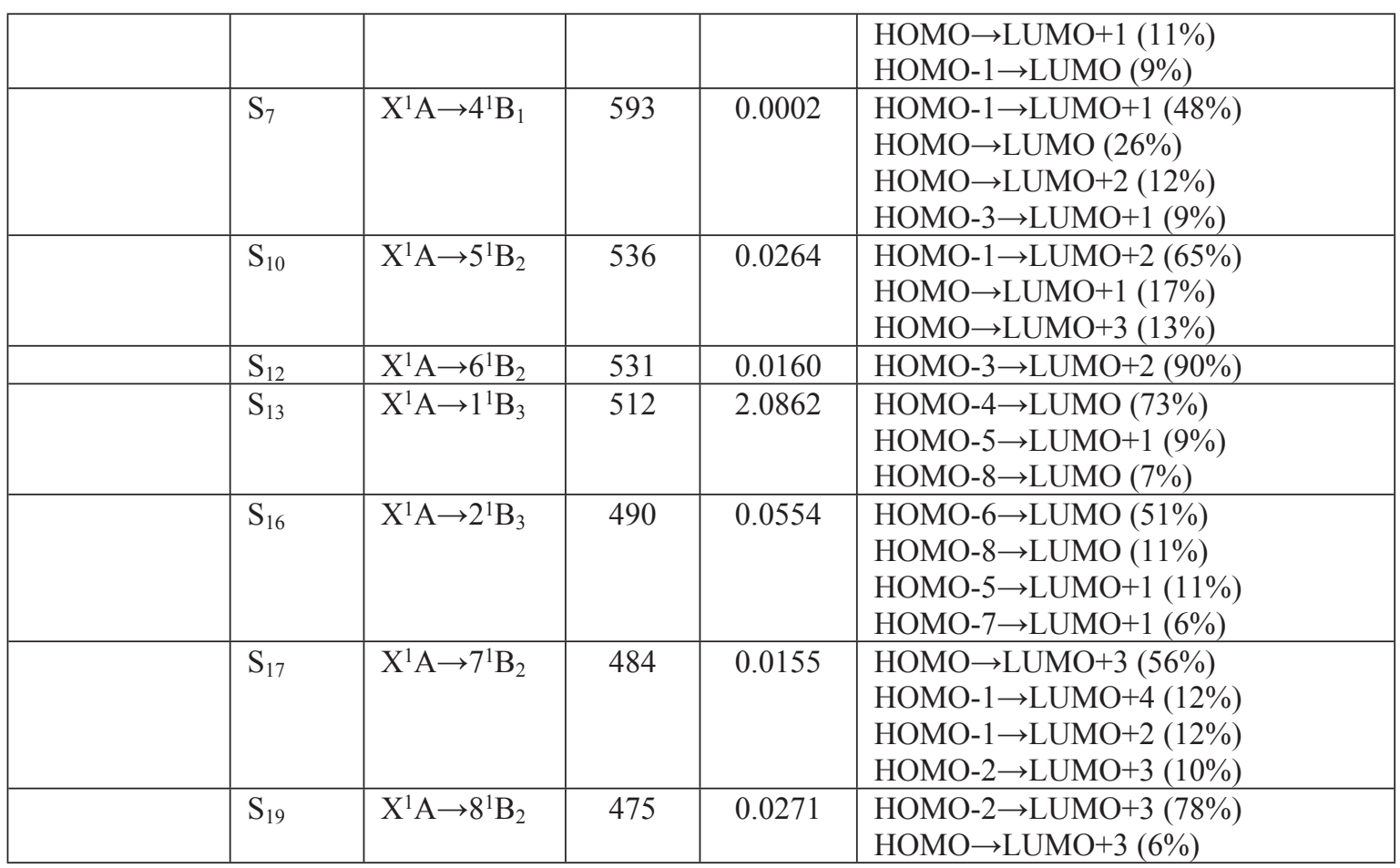

Table S2. Wavelengths $(\lambda)$, oscillator strengths $(f)$ and orbital assignment of the selected electronic transitions in the calculated absorption spectra of the TSC-based oligomers

\begin{tabular}{|c|c|c|c|c|c|}
\hline Compound & State & Transition & $\lambda \mathrm{nm}$ & $f$ & Assignment \\
\hline \multirow[t]{3}{*}{$\begin{array}{l}\text { TSC } \\
\left(D_{2 \mathrm{~d}} \text { symmetry }\right. \\
\text { point group }) \\
\end{array}$} & $\mathrm{S}_{1}$ & $\mathrm{X}^{1} \mathrm{~A} \rightarrow 1^{1} \mathrm{~A}_{2}$ & 429 & 0 & HOMO-3 $\rightarrow$ LUMO (99\%) \\
\hline & $\mathrm{S}_{2}$ & $\mathrm{X}^{1} \mathrm{~A} \rightarrow 1^{1} \mathrm{~A}_{1}$ & 420 & 0 & HOMO-2 $\rightarrow$ LUMO (98\%) \\
\hline & $\mathrm{S}_{3(4)}$ & $\mathrm{X}^{1} \mathrm{~A} \rightarrow 1^{1} \mathrm{E}$ & 415 & 0.1749 & $\begin{array}{l}\text { HOMO } \rightarrow \text { LUMO }(95 \%) \\
(\text { HOMO-1 } \rightarrow \text { LUMO }(95 \%))\end{array}$ \\
\hline \multicolumn{6}{|c|}{ TSCs (Type I) } \\
\hline \multirow{6}{*}{$\begin{array}{l}\mathrm{n}=2 \\
\left(C_{l} \text { symmetry }\right. \\
\text { point group) }\end{array}$} & $\mathrm{S}_{1}$ & $\mathrm{X}^{1} \mathrm{~A} \rightarrow 1^{1} \mathrm{~A}$ & 485 & 0.4157 & $\mathrm{HOMO} \rightarrow \mathrm{LUMO}(91 \%)$ \\
\hline & $\mathrm{S}_{2}$ & $\mathrm{X}^{1} \mathrm{~A} \rightarrow 2^{1} \mathrm{~A}$ & 476 & 0.0238 & $\begin{array}{l}\text { HOMO-1 } \rightarrow \text { LUMO }(80 \%) \\
\text { HOMO-5 } \rightarrow \text { LUMO }(14 \%)\end{array}$ \\
\hline & $\mathrm{S}_{3}$ & $\mathrm{X}^{1} \mathrm{~A} \rightarrow 3^{1} \mathrm{~A}$ & 441 & 0.0354 & HOMO-2 $\rightarrow$ LUMO (91\%) \\
\hline & $\mathrm{S}_{4}$ & $\mathrm{X}^{1} \mathrm{~A} \rightarrow 4^{1} \mathrm{~A}$ & 437 & 0.0642 & $\begin{array}{l}\text { HOMO-5 } \rightarrow \text { LUMO }(80 \%) \\
\text { HOMO-1 } \rightarrow \text { LUMO }(14 \%)\end{array}$ \\
\hline & $\mathrm{S}_{5}$ & $\mathrm{X}^{1} \mathrm{~A} \rightarrow 5^{1} \mathrm{~A}$ & 431 & 0.0750 & $\begin{array}{l}\text { HOMO-4 } \rightarrow \text { LUMO }(80 \%) \\
\text { HOMO-6 } \rightarrow \text { LUMO }(16 \%)\end{array}$ \\
\hline & $\mathrm{S}_{10}$ & $\mathrm{X}^{1} \mathrm{~A} \rightarrow 19^{1} \mathrm{~A}$ & 399 & 0.5084 & HOMO-1 $\rightarrow$ LUMO+2 (86\%) \\
\hline \multirow{6}{*}{$\begin{array}{l}\mathrm{n}=3 \\
\left(C_{2 v} \text { symmetry }\right. \\
\text { point group })\end{array}$} & $\mathrm{S}_{1}$ & $\mathrm{X}^{1} \mathrm{~A}_{1} \rightarrow 1^{1} \mathrm{~B}_{1}$ & 473 & 0.8103 & $\mathrm{HOMO} \rightarrow$ LUMO (93\%) \\
\hline & $\mathrm{S}_{2}$ & $\mathrm{X}^{1} \mathrm{~A}_{1} \rightarrow 1^{1} \mathrm{~A}_{1}$ & 445 & 0.0004 & HOMO-2 $\rightarrow$ LUMO (93\%) \\
\hline & $\mathrm{S}_{3}$ & $\mathrm{X}^{1} \mathrm{~A}_{1} \rightarrow 1^{1} \mathrm{~B}_{2}$ & 439 & 0.0905 & HOMO-1 $\rightarrow$ LUMO (84\%) \\
\hline & $\mathrm{S}_{4}$ & $\mathrm{X}^{1} \mathrm{~A}_{1} \rightarrow 2^{1} \mathrm{~A}_{1}$ & 436 & 0.0036 & $\begin{array}{l}\text { HOMO-3 } \rightarrow \text { LUMO }(45 \%) \\
\text { HOMO } \rightarrow \text { LUMO+1 }(26 \%) \\
\text { HOMO-6 } \rightarrow \text { LUMO }(10 \%)\end{array}$ \\
\hline & $\mathrm{S}_{6}$ & $\mathrm{X}^{1} \mathrm{~A}_{1} \rightarrow 2^{1} \mathrm{~B}_{1}$ & 430 & 0.0119 & $\begin{array}{l}\text { HOMO-4 } \rightarrow \text { LUMO }(76 \%) \\
\text { HOMO-2 } \rightarrow \text { LUMO+1 }(15 \%)\end{array}$ \\
\hline & $\mathrm{S}_{7}$ & $\mathrm{X}^{1} \mathrm{~A}_{1} \rightarrow 3^{1} \mathrm{~A}_{1}$ & 418 & 0.0121 & $\begin{array}{l}\text { HOMO-6 } \rightarrow \text { LUMO }(62 \%) \\
\text { HOMO-4 } \rightarrow \text { LUMO+1 }(13 \%) \\
\text { HOMO } \rightarrow \text { LUMO+1 }(9 \%)\end{array}$ \\
\hline
\end{tabular}




\begin{tabular}{|c|c|c|c|c|c|}
\hline & $\mathrm{S}_{10}$ & $\mathrm{X}^{1} \mathrm{~A}_{1} \rightarrow 2^{1} \mathrm{~B}_{2}$ & 410 & 0.0038 & $\begin{array}{l}\text { HOMO-2 } \rightarrow \text { LUMO+2 (81\%) } \\
\text { HOMO-4 } \rightarrow \text { LUMO+3 (9\%) }\end{array}$ \\
\hline & $\mathrm{S}_{11}$ & $\mathrm{X}^{1} \mathrm{~A}_{1} \rightarrow 5^{1} \mathrm{~A}_{1}$ & 407 & 0.0487 & $\begin{array}{l}\text { HOMO- } \rightarrow \text { LUMO+3 }(56 \%) \\
\text { HOMO-5 } \rightarrow \text { LUMO+2 }(30 \%)\end{array}$ \\
\hline & $\mathrm{S}_{12}$ & $\mathrm{X}^{1} \mathrm{~A}_{1} \rightarrow 3^{1} \mathrm{~B}_{1}$ & 406 & 0.3572 & $\begin{array}{l}\mathrm{HOMO}-3 \rightarrow \mathrm{LUMO}+1(45 \%) \\
\mathrm{HOMO} \rightarrow \mathrm{LUMO}+3(33 \%)\end{array}$ \\
\hline & $\mathrm{S}_{13}$ & $\mathrm{X}^{1} \mathrm{~A}_{1} \rightarrow 3^{1} \mathrm{~B}_{2}$ & 403 & 0.0440 & $\begin{array}{l}\mathrm{HOMO} \rightarrow \mathrm{LUMO}+3(79 \%) \\
\mathrm{HOMO}-1 \rightarrow \text { LUMO }(8 \%)\end{array}$ \\
\hline & $\mathrm{S}_{15}$ & $\mathrm{X}^{1} \mathrm{~A}_{1} \rightarrow 4^{1} \mathrm{~B}_{1}$ & 400 & 0.0730 & $\begin{array}{l}\text { HOMO-7 } \rightarrow \text { LUMO (49\%) } \\
\text { HOMO-2 } \rightarrow \text { LUMO+1 (18\%) }\end{array}$ \\
\hline & $\mathrm{S}_{20}$ & $\mathrm{X}^{1} \mathrm{~A}_{1} \rightarrow 6^{1} \mathrm{~B}_{1}$ & 384 & 0.0780 & $\begin{array}{l}\text { HOMO-3 } \rightarrow \text { LUMO+1 }(57 \%) \\
\text { HOMO-7 } \rightarrow \text { LUMO }(16 \%)\end{array}$ \\
\hline \multirow{7}{*}{$\begin{array}{l}\mathrm{n}=4 \\
\left(C_{s} \text { symmetry }\right. \\
\text { point group) }\end{array}$} & $\mathrm{S}_{1}$ & $\mathrm{X}^{1} \mathrm{~A}^{\prime} \rightarrow 1^{1} \mathrm{~A}^{\prime}$ & 483 & 1.3109 & $\mathrm{HOMO} \rightarrow$ LUMO $(90 \%)$ \\
\hline & $\mathrm{S}_{2}$ & $\mathrm{X}^{1} \mathrm{~A}^{\prime} \rightarrow 2^{1} \mathrm{~A}^{\prime}$ & 453 & 0 & $\begin{array}{l}\text { HOMO-1 } \rightarrow \text { LUMO }(56 \%) \\
\text { HOMO } \rightarrow \text { LUMO+1 }(32 \%)\end{array}$ \\
\hline & $\mathrm{S}_{4}$ & $\mathrm{X}^{1} \mathrm{~A}^{\prime} \rightarrow 1^{1} \mathrm{~A}^{\prime \prime}$ & 441 & 0.1013 & HOMO-2 $\rightarrow$ LUMO $(81 \%)$ \\
\hline & $\mathrm{S}_{8}$ & $\mathrm{X}^{1} \mathrm{~A}^{\prime} \rightarrow 5^{1} \mathrm{~A}^{\prime}$ & 430 & 0.0422 & $\begin{array}{l}\text { HOMO-7 } \rightarrow \text { LUMO }(27 \%) \\
\text { HOMO-1 } \rightarrow \text { LUMO+1 }(21 \%) \\
\text { HOMO-8 } \rightarrow \text { LUMO }(15 \%)\end{array}$ \\
\hline & $\mathrm{S}_{11}$ & $\mathrm{X}^{1} \mathrm{~A}^{\prime} \rightarrow 8^{1} \mathrm{~A}^{\prime}$ & 416 & 0.1884 & $\begin{array}{l}\text { HOMO-8 } \rightarrow \text { LUMO }(45 \%) \\
\text { HOMO-1 } \rightarrow \text { LUMO+1 }(18 \%) \\
\text { HOMO-6 } \rightarrow \text { LUMO+1 }(18 \%)\end{array}$ \\
\hline & $\mathrm{S}_{12}$ & $\mathrm{X}^{1} \mathrm{~A}^{\prime} \rightarrow 4^{1} \mathrm{~A}^{\prime \prime}$ & 412 & 0.0136 & $\begin{array}{l}\text { HOMO-9 } \rightarrow \text { LUMO }(39 \%) \\
\text { HOMO } \rightarrow \text { LUMO+2 }(32 \%)\end{array}$ \\
\hline & $\mathrm{S}_{15}$ & $\mathrm{X}^{1} \mathrm{~A}^{\prime} \rightarrow 9^{1} \mathrm{~A}^{\prime}$ & 408 & 0.1520 & $\begin{array}{l}\mathrm{HOMO}-2 \rightarrow \mathrm{LUMO}+2(54 \%) \\
\mathrm{HOMO}-5 \rightarrow \mathrm{LUMO}+3(15 \%)\end{array}$ \\
\hline \multirow{4}{*}{$\begin{array}{l}\mathrm{n}=5 \\
\left(C_{2 v} \text { symmetry }\right. \\
\text { point group })\end{array}$} & $\mathrm{S}_{1}$ & $\mathrm{X}^{1} \mathrm{~A}_{1} \rightarrow 1^{1} \mathrm{~B}_{1}$ & 488 & 1.8603 & $\mathrm{HOMO} \rightarrow \mathrm{LUMO}(88 \%)$ \\
\hline & $\mathrm{S}_{2}$ & $\mathrm{X}^{1} \mathrm{~A}_{1} \rightarrow 1^{1} \mathrm{~A}_{1}$ & 464 & 0.0034 & $\begin{array}{l}\text { HOMO-1 } \rightarrow \text { LUMO }(53 \%) \\
\text { HOMO } \rightarrow \text { LUMO+1 }(38 \%)\end{array}$ \\
\hline & $\mathrm{S}_{5}$ & $\mathrm{X}^{1} \mathrm{~A}_{1} \rightarrow 1^{1} \mathrm{~B}_{2}$ & 442 & 0.1200 & $\begin{array}{l}\text { HOMO-2 } \rightarrow \text { LUMO (76\%) } \\
\text { HOMO-5 } \rightarrow \text { LUMO+1 (8\%) }\end{array}$ \\
\hline & $\mathrm{S}_{6}$ & $\mathrm{X}^{1} \mathrm{~A}_{1} \rightarrow 3^{1} \mathrm{~B}_{1}$ & 441 & 0.0349 & $\begin{array}{l}\text { HOMO-6 } \rightarrow \text { LUMO }(76 \%) \\
\text { HOMO-3 } \rightarrow \text { LUMO+1 }(20 \%)\end{array}$ \\
\hline \multicolumn{6}{|c|}{ TSCs (Type II) } \\
\hline \multirow{8}{*}{$\begin{array}{l}\mathrm{n}=2 \\
\left(C_{l} \text { symmetry }\right. \\
\text { point group) }\end{array}$} & $\mathrm{S}_{1}$ & $\mathrm{X}^{1} \mathrm{~A} \rightarrow 1^{1} \mathrm{~A}$ & 497 & 0.0437 & $\mathrm{HOMO} \rightarrow \mathrm{LUMO}(96 \%)$ \\
\hline & $\mathrm{S}_{2}$ & $\mathrm{X}^{1} \mathrm{~A} \rightarrow 2^{1} \mathrm{~A}$ & 486 & 0.2867 & $\begin{array}{l}\text { HOMO } \rightarrow \text { LUMO+1 }(81 \%) \\
\text { HOMO-1 } \rightarrow \text { LUMO }(9 \%)\end{array}$ \\
\hline & $\mathrm{S}_{3}$ & $\mathrm{X}^{1} \mathrm{~A} \rightarrow 3^{1} \mathrm{~A}$ & 443 & 0.6007 & $\begin{array}{l}\text { HOMO-1 } \rightarrow \text { LUMO }(85 \%) \\
\text { HOMO } \rightarrow \text { LUMO+1 }(9 \%)\end{array}$ \\
\hline & $\mathrm{S}_{6}$ & $\mathrm{X}^{1} \mathrm{~A} \rightarrow 6^{1} \mathrm{~A}$ & 433 & 0.0151 & $\begin{array}{l}\text { HOMO-4 } \rightarrow \text { LUMO (79\%) } \\
\text { HOMO-1 } \rightarrow \text { LUMO+1 (8\%) } \\
\text { HOMO-6 } \rightarrow \text { LUMO (7\%) }\end{array}$ \\
\hline & $\mathrm{S}_{8}$ & $\mathrm{X}^{1} \mathrm{~A} \rightarrow 8^{1} \mathrm{~A}$ & 426 & 0.0563 & $\begin{array}{l}\text { HOMO-4 } \rightarrow \text { LUMO+1 }(52 \%) \\
\text { HOMO-6 } \rightarrow \text { LUMO+1 }(18 \%) \\
\text { HOMO-1 } \rightarrow \text { LUMO+1 }(17 \%)\end{array}$ \\
\hline & $\mathrm{S}_{9}$ & $\mathrm{X}^{1} \mathrm{~A} \rightarrow 9^{1} \mathrm{~A}$ & 420 & 0.3575 & $\begin{array}{l}\text { HOMO-6 } \rightarrow \text { LUMO }(53 \%) \\
\text { HOMO-6 } \rightarrow \text { LUMO+1 }(29 \%)\end{array}$ \\
\hline & $\mathrm{S}_{12}$ & $\mathrm{X}^{1} \mathrm{~A} \rightarrow 12^{1} \mathrm{~A}$ & 413 & 0.2573 & $\begin{array}{l}\mathrm{HOMO}-1 \rightarrow \text { LUMO+1 }(67 \%) \\
\text { HOMO-4 } \rightarrow \text { LUMO+1 }(13 \%) \\
\text { HOMO-4 } \rightarrow \text { LUMO }(12 \%)\end{array}$ \\
\hline & $\mathrm{S}_{15}$ & $\mathrm{X}^{1} \mathrm{~A} \rightarrow 15^{1} \mathrm{~A}$ & 401 & 0.5448 & $\begin{array}{l}\text { HOMO-6 } \rightarrow \text { LUMO+1 (41\%) } \\
\text { HOMO-6 } \rightarrow \text { LUMO }(29 \%) \\
\text { HOMO-4 } \rightarrow \text { LUMO+1 }(17 \%)\end{array}$ \\
\hline $\begin{array}{l}\mathrm{n}=3 \\
\left(C_{2} \text { symmetry }\right.\end{array}$ & $\mathrm{S}_{1}$ & $\mathrm{X}^{1} \mathrm{~A} \rightarrow 1^{1} \mathrm{~A}$ & 527 & 0 & $\mathrm{HOMO} \rightarrow$ LUMO (97\%) \\
\hline
\end{tabular}




\begin{tabular}{|c|c|c|c|c|c|}
\hline \multicolumn{6}{|l|}{ point group) } \\
\hline & $\mathrm{S}_{2}$ & $\mathrm{X}^{1} \mathrm{~A} \rightarrow 1^{1} \mathrm{~B}$ & 502 & 0.1407 & $\mathrm{HOMO} \rightarrow \mathrm{LUMO}+1(91 \%)$ \\
\hline & $\mathrm{S}_{3}$ & $\mathrm{X}^{1} \mathrm{~A} \rightarrow 2^{1} \mathrm{~B}$ & 500 & 0.7857 & $\mathrm{HOMO} \rightarrow \mathrm{LUMO}+2(88 \%)$ \\
\hline & $\mathrm{S}_{4}$ & $\mathrm{X}^{1} \mathrm{~A} \rightarrow 2^{1} \mathrm{~A}$ & 471 & 0.0063 & $\begin{array}{l}\mathrm{HOMO} \rightarrow \text { LUMO+3 }(48 \%) \\
\mathrm{HOMO}-1 \rightarrow \text { LUMO+2 }(16 \%) \\
\mathrm{HOMO}-2 \rightarrow \text { LUMO }(14 \%)\end{array}$ \\
\hline & $\mathrm{S}_{7}$ & $\mathrm{X}^{1} \mathrm{~A} \rightarrow 4^{1} \mathrm{~B}$ & 446 & 0.9895 & $\begin{array}{l}\text { HOMO-3 } \rightarrow \text { LUMO }(52 \%) \\
\text { HOMO-5 } \rightarrow \text { LUMO }(17 \%) \\
\text { HOMO-2 } \rightarrow \text { LUMO+1 }(17 \%)\end{array}$ \\
\hline & $\mathrm{S}_{8}$ & $\mathrm{X}^{1} \mathrm{~A} \rightarrow 4^{1} \mathrm{~A}$ & 441 & 0.0572 & $\begin{array}{l}\text { HOMO-2 } \rightarrow \text { LUMO (53\%) } \\
\text { HOMO } \rightarrow \text { LUMO+3 }(30 \%) \\
\text { HOMO-3 } \rightarrow \text { LUMO+1 }(8 \%)\end{array}$ \\
\hline & $\mathrm{S}_{9}$ & $\mathrm{X}^{1} \mathrm{~A} \rightarrow 5^{1} \mathrm{~B}$ & 440 & 0.2555 & $\begin{array}{l}\text { HOMO-5 } \rightarrow \text { LUMO }(69 \%) \\
\text { HOMO-2 } \rightarrow \text { LUMO+1 }(13 \%) \\
\text { HOMO-9 } \rightarrow \text { LUMO }(6 \%)\end{array}$ \\
\hline & $\mathrm{S}_{11}$ & $\mathrm{X}^{1} \mathrm{~A} \rightarrow 6^{1} \mathrm{~B}$ & 434 & 0.0852 & $\begin{array}{l}\text { HOMO-7 } \rightarrow \text { LUMO }(58 \%) \\
\text { HOMO-6 } \rightarrow \text { LUMO+2 (8\%) } \\
\text { HOMO-4 } \rightarrow \text { LUMO+1 }(7 \%) \\
\text { HOMO-6 } \rightarrow \text { LUMO+1 }(7 \%)\end{array}$ \\
\hline & $\mathrm{S}_{12}$ & $\mathrm{X}^{1} \mathrm{~A} \rightarrow 6^{1} \mathrm{~A}$ & 433 & 0.0210 & $\begin{array}{l}\text { HOMO-8 } \rightarrow \text { LUMO }(21 \%) \\
\text { HOMO-1 } \rightarrow \text { LUMO+2 }(21 \%) \\
\text { HOMO-4 } \rightarrow \text { LUMO }(18 \%) \\
\text { HOMO-7 } \rightarrow \text { LUMO+1 }(12 \%) \\
\text { HOMO-6 } \rightarrow \text { LUMO }(6 \%)\end{array}$ \\
\hline & $\mathrm{S}_{14}$ & $\mathrm{X}^{1} \mathrm{~A} \rightarrow 7^{1} \mathrm{~B}$ & 426 & 0.4466 & $\begin{array}{l}\text { HOMO-9 } \rightarrow \text { LUMO }(31 \%) \\
\text { HOMO-8 } \rightarrow \text { LUMO+1 }(23 \%) \\
\text { HOMO-3 } \rightarrow \text { LUMO }(11 \%) \\
\text { HOMO-6 } \rightarrow \text { LUMO+2 }(6 \%) \\
\text { HOMO-6 } \rightarrow \text { LUMO+1 }(6 \%)\end{array}$ \\
\hline & $\mathrm{S}_{15}$ & $\mathrm{X}^{1} \mathrm{~A} \rightarrow 8^{1} \mathrm{~A}$ & 424 & 0.0422 & $\begin{array}{l}\text { HOMO-6 } \rightarrow \text { LUMO }(27 \%) \\
\text { HOMO-8 } \rightarrow \text { LUMO }(22 \%) \\
\text { HOMO-3 } \rightarrow \text { LUMO+1 }(11 \%) \\
\text { HOMO-1 } \rightarrow \text { LUMO+2 }(10 \%) \\
\text { HOMO-7 } \rightarrow \text { LUMO+2 }(6 \%) \\
\text { HOMO-7 } \rightarrow \text { LUMO+1 }(6 \%)\end{array}$ \\
\hline & $\mathrm{S}_{16}$ & $\mathrm{X}^{1} \mathrm{~A} \rightarrow 8^{1} \mathrm{~B}$ & 419 & 0.0568 & $\begin{array}{l}\mathrm{HOMO}-1 \rightarrow \text { LUMO+3 }(37 \%) \\
\mathrm{HOMO}-4 \rightarrow \mathrm{LUMO}+2(23 \%) \\
\mathrm{HOMO}-6 \rightarrow \mathrm{LUMO}+2(9 \%) \\
\mathrm{HOMO}-7 \rightarrow \text { LUMO+3 }(7 \%) \\
\mathrm{HOMO}-4 \rightarrow \mathrm{LUMO}+1(6 \%)\end{array}$ \\
\hline & $\mathrm{S}_{18}$ & $\mathrm{X}^{1} \mathrm{~A} \rightarrow 9^{1} \mathrm{~B}$ & 417 & 0.1874 & $\begin{array}{l}\mathrm{HOMO}-6 \rightarrow \text { LUMO+2 }(27 \%) \\
\mathrm{HOMO}-1 \rightarrow \text { LUMO+3 }(18 \%) \\
\mathrm{HOMO}-9 \rightarrow \text { LUMO+3 }(12 \%) \\
\mathrm{HOMO}-6 \rightarrow \text { LUMO+1 }(11 \%) \\
\mathrm{HOMO}-8 \rightarrow \text { LUMO+2 }(6 \%)\end{array}$ \\
\hline \multirow[t]{6}{*}{$\begin{array}{l}\mathrm{n}=4 \\
\left(C_{l} \text { symmetry }\right. \\
\text { point group })\end{array}$} & $\mathrm{S}_{1}$ & $\mathrm{X}^{1} \mathrm{~A} \rightarrow 1^{1} \mathrm{~A}$ & 535 & 0.0001 & HOMO $\rightarrow$ LUMO (93\%) \\
\hline & $\mathrm{S}_{2}$ & $\mathrm{X}^{1} \mathrm{~A} \rightarrow 2^{1} \mathrm{~A}$ & 518 & 0.0083 & $\begin{array}{l}\text { HOMO } \rightarrow \text { LUMO+1 (78\%) } \\
\text { HOMO-1 } \rightarrow \text { LUMO }(17 \%)\end{array}$ \\
\hline & $\mathrm{S}_{3}$ & $\mathrm{X}^{1} \mathrm{~A} \rightarrow 3^{1} \mathrm{~A}$ & 503 & 0.0173 & $\begin{array}{l}\mathrm{HOMO} \rightarrow \mathrm{LUMO}+3(78 \%) \\
\mathrm{HOMO}-1 \rightarrow \mathrm{LUMO}+1(17 \%)\end{array}$ \\
\hline & $\mathrm{S}_{4}$ & $\mathrm{X}^{1} \mathrm{~A} \rightarrow 4^{1} \mathrm{~A}$ & 502 & 1.6174 & $\begin{array}{l}\mathrm{HOMO} \rightarrow \mathrm{LUMO}+2(81 \%) \\
\mathrm{HOMO}-1 \rightarrow \mathrm{LUMO}+4(11 \%)\end{array}$ \\
\hline & $\mathrm{S}_{9}$ & $\mathrm{X}^{1} \mathrm{~A} \rightarrow 9^{1} \mathrm{~A}$ & 458 & 0.0453 & $\begin{array}{l}\text { HOMO- } \rightarrow \text { LUMO+4 }(21 \%) \\
\text { HOMO } \rightarrow \text { LUMO+5 }(15 \%) \\
\text { HOMO-2 } \rightarrow \text { LUMO }+2(11 \%) \\
\text { HOMO-8 } \rightarrow \text { LUMO }(9 \%)\end{array}$ \\
\hline & $\mathrm{S}_{12}$ & $\mathrm{X}^{1} \mathrm{~A} \rightarrow 12^{1} \mathrm{~A}$ & 444 & 0.2378 & $\begin{array}{l}\text { HOMO-5 } \rightarrow \text { LUMO }(37 \%) \\
\text { HOMO-7 } \rightarrow \text { LUMO }(26 \%) \\
\text { HOMO-6 } \rightarrow \text { LUMO+1 }(13 \%) \\
\text { HOMO-4 } \rightarrow \text { LUMO+1 }(6 \%)\end{array}$ \\
\hline
\end{tabular}




\begin{tabular}{|c|c|c|c|c|c|}
\hline & $\mathrm{S}_{14}$ & $\mathrm{X}^{1} \mathrm{~A} \rightarrow 14^{1} \mathrm{~A}$ & 442 & 1.4065 & $\begin{array}{l}\text { HOMO-4 } \rightarrow \text { LUMO+1 }(25 \%) \\
\text { HOMO-3 } \rightarrow \text { LUMO+3 }(15 \%) \\
\text { HOMO-8 } \rightarrow \text { LUMO }(10 \%) \\
\text { HOMO-3 } \rightarrow \text { LUMO }(9 \%) \\
\text { HOMO-12 } \rightarrow \text { LUMO }(9 \%)\end{array}$ \\
\hline & $\mathrm{S}_{19}$ & $\mathrm{X}^{1} \mathrm{~A} \rightarrow 19^{1} \mathrm{~A}$ & 432 & 0.5003 & $\begin{array}{l}\text { HOMO-9 } \rightarrow \text { LUMO+1 }(24 \%) \\
\text { HOMO-10 } \rightarrow \text { LUMO }(20 \%) \\
\text { HOMO-7 } \rightarrow \text { LUMO+3 }(8 \%) \\
\text { HOMO-5 } \rightarrow \text { LUMO }(6 \%)\end{array}$ \\
\hline \multirow{14}{*}{$\begin{array}{l}\mathrm{n}=5 \\
\left(C_{2} \text { symmetry }\right. \\
\text { point group })\end{array}$} & $\mathrm{S}_{1}$ & $\mathrm{X}^{1} \mathrm{~A} \rightarrow 1^{1} \mathrm{~A}$ & 540 & 0 & $\begin{array}{l}\mathrm{HOMO} \rightarrow \mathrm{LUMO}(88 \%) \\
\mathrm{HOMO}-1 \rightarrow \mathrm{LUMO}+1(7 \%)\end{array}$ \\
\hline & $\mathrm{S}_{2}$ & $\mathrm{X}^{1} \mathrm{~A} \rightarrow 1^{1} \mathrm{~B}$ & 528 & 0.0088 & $\begin{array}{l}\mathrm{HOMO} \rightarrow \text { LUMO+1 }(65 \%) \\
\text { HOMO-1 } \rightarrow \text { LUMO }(27 \%)\end{array}$ \\
\hline & $\mathrm{S}_{4}$ & $\mathrm{X}^{1} \mathrm{~A} \rightarrow 2^{1} \mathrm{~B}$ & 505 & 2.5225 & $\begin{array}{l}\mathrm{HOMO} \rightarrow \mathrm{LUMO}+3(70 \%) \\
\mathrm{HOMO}-1 \rightarrow \mathrm{LUMO}+5(15 \%) \\
\mathrm{HOMO} \rightarrow \mathrm{LUMO}+6(7 \%)\end{array}$ \\
\hline & $\mathrm{S}_{5}$ & $\mathrm{X}^{1} \mathrm{~A} \rightarrow 3^{1} \mathrm{~B}$ & 503 & 0.0526 & $\begin{array}{l}\mathrm{HOMO} \rightarrow \mathrm{LUMO}+4(63 \%) \\
\mathrm{HOMO}-1 \rightarrow \mathrm{LUMO}+2(27 \%) \\
\mathrm{HOMO}-2 \rightarrow \mathrm{LUMO}+1(6 \%)\end{array}$ \\
\hline & $\mathrm{S}_{6}$ & $\mathrm{X}^{1} \mathrm{~A} \rightarrow 4^{1} \mathrm{~B}$ & 496 & 0.0103 & $\begin{array}{l}\text { HOMO-1 } \rightarrow \text { LUMO (61\%) } \\
\text { HOMO } \rightarrow \text { LUMO+1 }(21 \%) \\
\text { HOMO-2 } \rightarrow \text { LUMO+1 }(6 \%)\end{array}$ \\
\hline & $\mathrm{S}_{7}$ & $\mathrm{X}^{1} \mathrm{~A} \rightarrow 3^{1} \mathrm{~A}$ & 490 & 0.0102 & $\begin{array}{l}\mathrm{HOMO} \rightarrow \mathrm{LUMO}+5(44 \%) \\
\mathrm{HOMO}-1 \rightarrow \mathrm{LUMO}+3(38 \%) \\
\mathrm{HOMO}-2 \rightarrow \mathrm{LUMO}+5(5 \%)\end{array}$ \\
\hline & $\mathrm{S}_{9}$ & $\mathrm{X}^{1} \mathrm{~A} \rightarrow 5^{1} \mathrm{~B}$ & 470 & 0.0084 & $\begin{array}{l}\mathrm{HOMO} \rightarrow \mathrm{LUMO}+6(23 \%) \\
\mathrm{HOMO}-1 \rightarrow \mathrm{LUMO}+5(21 \%) \\
\mathrm{HOMO}-2 \rightarrow \mathrm{LUMO}+3(16 \%)\end{array}$ \\
\hline & $\mathrm{S}_{10}$ & $\mathrm{X}^{1} \mathrm{~A} \rightarrow 6^{1} \mathrm{~B}$ & 469 & 0.0034 & $\begin{array}{l}\text { HOMO- } \rightarrow \text { LUMO }+2(26 \%) \\
\text { HOMO } \rightarrow \text { LUMO }+4(23 \%) \\
\text { HOMO-2 } \rightarrow \text { LUMO } 1(16 \%) \\
\text { HOMO-3 } \rightarrow \text { LUMO }(8 \%)\end{array}$ \\
\hline & $\mathrm{S}_{13}$ & $\mathrm{X}^{1} \mathrm{~A} \rightarrow 7^{1} \mathrm{~B}$ & 456 & 0.0048 & $\begin{array}{l}\mathrm{HOMO}-2 \rightarrow \mathrm{LUMO}+1(39 \%) \\
\mathrm{HOMO}-1 \rightarrow \mathrm{LUMO}+2(22 \%) \\
\mathrm{HOMO}-3 \rightarrow \text { LUMO }(13 \%) \\
\mathrm{HOMO} \rightarrow \mathrm{LUMO}+1(6 \%)\end{array}$ \\
\hline & $\mathrm{S}_{14}$ & $\mathrm{X}^{1} \mathrm{~A} \rightarrow 7^{1} \mathrm{~A}$ & 456 & 0.0038 & $\begin{array}{l}\mathrm{HOMO} \rightarrow \mathrm{LUMO}+5(31 \%) \\
\mathrm{HOMO}-2 \rightarrow \mathrm{LUMO}+5(14 \%) \\
\mathrm{HOMO}-1 \rightarrow \mathrm{LUMO}+3(13 \%) \\
\mathrm{HOMO}-4 \rightarrow \mathrm{LUMO}(9 \%)\end{array}$ \\
\hline & $\mathrm{S}_{15}$ & $\mathrm{X}^{1} \mathrm{~A} \rightarrow 8^{1} \mathrm{~A}$ & 452 & 0.0071 & $\begin{array}{l}\mathrm{HOMO}-1 \rightarrow \mathrm{LUMO}+3(24 \%) \\
\mathrm{HOMO} \rightarrow \mathrm{LUMO}+5(20 \%) \\
\mathrm{HOMO}-1 \rightarrow \mathrm{LUMO}+6(16 \%) \\
\text { HOMO-4 } \rightarrow \text { LUMO }(7 \%)\end{array}$ \\
\hline & $\mathrm{S}_{16}$ & $\mathrm{X}^{1} \mathrm{~A} \rightarrow 8^{1} \mathrm{~B}$ & 445 & 0.0310 & $\begin{array}{l}\text { HOMO-7 } \rightarrow \text { LUMO }(50 \%) \\
\text { HOMO-3 } \rightarrow \text { LUMO+2 }(12 \%) \\
\text { HOMO-7 } \rightarrow \text { LUMO+2 }(7 \%) \\
\text { HOMO-2 } \rightarrow \text { LUMO+4 }(7 \%)\end{array}$ \\
\hline & $\mathrm{S}_{17}$ & $\mathrm{X}^{1} \mathrm{~A} \rightarrow 9^{1} \mathrm{~B}$ & 445 & 1.0553 & $\begin{array}{l}\text { HOMO-8 } \rightarrow \text { LUMO }(26 \%) \\
\text { HOMO-6 } \rightarrow \text { LUMO+1 }(11 \%) \\
\text { HOMO-9 } \rightarrow \text { LUMO+1 }(9 \%) \\
\text { HOMO-5 } \rightarrow \text { LUMO+2 }(7 \%) \\
\text { HOMO-4 } \rightarrow \text { LUMO+1 }(6 \%) \\
\text { HOMO } \rightarrow \text { LUMO+6 }(6 \%)\end{array}$ \\
\hline & $\mathrm{S}_{20}$ & $\mathrm{X}^{1} \mathrm{~A} \rightarrow 10^{1} \mathrm{~B}$ & 443 & 0.1826 & $\begin{array}{l}\mathrm{HOMO} \rightarrow \mathrm{LUMO}+6(37 \%) \\
\mathrm{HOMO} \rightarrow \mathrm{LUMO}+3(20 \%) \\
\mathrm{HOMO}-1 \rightarrow \mathrm{LUMO}+5(17 \%) \\
\mathrm{HOMO}-8 \rightarrow \mathrm{LUMO}(7 \%)\end{array}$ \\
\hline \multicolumn{6}{|c|}{ TSCs (Type III) } \\
\hline $\begin{array}{l}\mathrm{n}=2 \\
\left(C_{l} \text { symmetry }\right.\end{array}$ & $\mathrm{S}_{1}$ & $\mathrm{X}^{1} \mathrm{~A} \rightarrow 1^{1} \mathrm{~A}$ & 695 & 0.0079 & $\mathrm{HOMO} \rightarrow \mathrm{LUMO}(98 \%)$ \\
\hline
\end{tabular}




\begin{tabular}{|c|c|c|c|c|c|}
\hline \multicolumn{6}{|l|}{ point group) } \\
\hline & $\mathrm{S}_{2}$ & $\mathrm{X}^{1} \mathrm{~A} \rightarrow 2^{1} \mathrm{~A}$ & 528 & 0.0343 & $\begin{array}{l}\mathrm{HOMO} \rightarrow \text { LUMO+1 }(89 \%) \\
\text { HOMO-5 } \rightarrow \text { LUMO }(6 \%)\end{array}$ \\
\hline & $\mathrm{S}_{3}$ & $\mathrm{X}^{1} \mathrm{~A} \rightarrow 3^{1} \mathrm{~A}$ & 508 & 0.2631 & $\begin{array}{l}\text { HOMO- } \rightarrow \text { LUMO }(79 \%) \\
\text { HOMO-2 } \rightarrow \text { LUMO }(16 \%)\end{array}$ \\
\hline & $\mathrm{S}_{4}$ & $\mathrm{X}^{1} \mathrm{~A} \rightarrow 4^{1} \mathrm{~A}$ & 487 & 0.0090 & HOMO-4 $\rightarrow$ LUMO (94\%) \\
\hline & $\mathrm{S}_{5}$ & $\mathrm{X}^{1} \mathrm{~A} \rightarrow 5^{1} \mathrm{~A}$ & 482 & 0.2060 & $\begin{array}{l}\text { HOMO-2 } \rightarrow \text { LUMO }(80 \%) \\
\text { HOMO- } 1 \rightarrow \text { LUMO }(16 \%)\end{array}$ \\
\hline & $\mathrm{S}_{7}$ & $\mathrm{X}^{1} \mathrm{~A} \rightarrow 7^{1} \mathrm{~A}$ & 479 & 0.0732 & $\begin{array}{l}\text { HOMO-5 } \rightarrow \text { LUMO }(79 \%) \\
\text { HOMO } \rightarrow \text { LUMO }+1(9 \%)\end{array}$ \\
\hline & $\mathrm{S}_{9}$ & $\mathrm{X}^{1} \mathrm{~A} \rightarrow 9^{1} \mathrm{~A}$ & 441 & 0.0401 & $\begin{array}{l}\text { HOMO-7 } \rightarrow \text { LUMO }(82 \%) \\
\text { HOMO-5 } \rightarrow \text { LUMO }(10 \%)\end{array}$ \\
\hline & $\mathrm{S}_{11}$ & $\mathrm{X}^{1} \mathrm{~A} \rightarrow 11^{1} \mathrm{~A}$ & 395 & 0.3739 & $\begin{array}{l}\text { HOMO-3 } \rightarrow \text { LUMO+1 }(61 \%) \\
\text { HOMO } \rightarrow \text { LUMO+2 }(33 \%)\end{array}$ \\
\hline & $\mathrm{S}_{13}$ & $\mathrm{X}^{1} \mathrm{~A} \rightarrow 13^{1} \mathrm{~A}$ & 388 & 0.0220 & $\begin{array}{l}\text { HOMO-5 } \rightarrow \text { LUMO+1 (74\%) } \\
\text { HOMO } \rightarrow \text { LUMO+3 }(20 \%)\end{array}$ \\
\hline & $\mathrm{S}_{14}$ & $\mathrm{X}^{1} \mathrm{~A} \rightarrow 14^{1} \mathrm{~A}$ & 388 & 0.0676 & $\begin{array}{l}\text { HOMO-4 } \rightarrow \text { LUMO+1 }(84 \%) \\
\text { HOMO-7 } \rightarrow \text { LUMO }(6 \%)\end{array}$ \\
\hline & $\mathrm{S}_{15}$ & $\mathrm{X}^{1} \mathrm{~A} \rightarrow 15^{1} \mathrm{~A}$ & 387 & 0.5168 & $\begin{array}{l}\text { HOMO } \rightarrow \text { LUMO+2 (54\%) } \\
\text { HOMO-3 } \rightarrow \text { LUMO+1 }(30 \%) \\
\text { HOMO-6 } \rightarrow \text { LUMO+1 }(9 \%)\end{array}$ \\
\hline & $\mathrm{S}_{17}$ & $\mathrm{X}^{1} \mathrm{~A} \rightarrow 17^{1} \mathrm{~A}$ & 383 & 0.0109 & $\mathrm{HOMO} \rightarrow \mathrm{LUMO}+4(91 \%)$ \\
\hline & $\mathrm{S}_{18}$ & $\mathrm{X}^{1} \mathrm{~A} \rightarrow 18^{1} \mathrm{~A}$ & 374 & 0.0020 & HOMO-6 $\rightarrow$ LUMO+1 (87\%) \\
\hline & $\mathrm{S}_{19}$ & $\mathrm{X}^{1} \mathrm{~A} \rightarrow 19^{1} \mathrm{~A}$ & 365 & 0.0107 & $\begin{array}{l}\mathrm{HOMO}-7 \rightarrow \mathrm{LUMO}+1(64 \%) \\
\mathrm{HOMO} \rightarrow \mathrm{LUMO}+3(25 \%)\end{array}$ \\
\hline \multirow{10}{*}{$\begin{array}{l}\mathrm{n}=3 \\
\left(C_{2} \text { symmetry }\right. \\
\text { point group })\end{array}$} & $\mathrm{S}_{1}$ & $\mathrm{X}^{1} \mathrm{~A} \rightarrow 1^{1} \mathrm{~B}$ & 779 & 0 & $\mathrm{HOMO} \rightarrow \mathrm{LUMO}(95 \%)$ \\
\hline & $\mathrm{S}_{2}$ & $\mathrm{X}^{1} \mathrm{~A} \rightarrow 1^{1} \mathrm{~A}$ & 718 & 0.0004 & HOMO-1 $\rightarrow$ LUMO (94\%) \\
\hline & $\mathrm{S}_{3}$ & $\mathrm{X}^{1} \mathrm{~A} \rightarrow 2^{1} \mathrm{~A}$ & 595 & 0.0359 & $\mathrm{HOMO} \rightarrow \mathrm{LUMO}+1(89 \%)$ \\
\hline & $\mathrm{S}_{4}$ & $\mathrm{X}^{1} \mathrm{~A} \rightarrow 2^{1} \mathrm{~B}$ & 586 & 0.0003 & $\begin{array}{l}\mathrm{HOMO}-1 \rightarrow \mathrm{LUMO}+1(88 \%) \\
\mathrm{HOMO} \rightarrow \mathrm{LUMO}+2(8 \%)\end{array}$ \\
\hline & $\mathrm{S}_{5}$ & $\mathrm{X}^{1} \mathrm{~A} \rightarrow 3^{1} \mathrm{~B}$ & 540 & 0.7386 & HOMO-2 $\rightarrow$ LUMO (91\%) \\
\hline & $\mathrm{S}_{8}$ & $\mathrm{X}^{1} \mathrm{~A} \rightarrow 4^{1} \mathrm{~A}$ & 499 & 0.0056 & $\begin{array}{l}\text { HOMO-7 } \rightarrow \text { LUMO }(67 \%) \\
\text { HOMO- } \rightarrow \text { LUMO+2 }(14 \%) \\
\text { HOMO-6 } \rightarrow \text { LUMO+1 }(12 \%)\end{array}$ \\
\hline & $\mathrm{S}_{10}$ & $\mathrm{X}^{1} \mathrm{~A} \rightarrow 6^{1} \mathrm{~B}$ & 497 & 0.1529 & $\begin{array}{l}\text { HOMO-5 } \rightarrow \text { LUMO }(84 \%) \\
\text { HOMO-4 } \rightarrow \text { LUMO+1 (7\%) }\end{array}$ \\
\hline & $\mathrm{S}_{12}$ & $\mathrm{X}^{1} \mathrm{~A} \rightarrow 6^{1} \mathrm{~A}$ & 486 & 0.0821 & $\begin{array}{l}\text { HOMO- } 1 \rightarrow \text { LUMO+2 }(78 \%) \\
\text { HOMO-7 } \rightarrow \text { LUMO }(13 \%)\end{array}$ \\
\hline & $\mathrm{S}_{15}$ & $\mathrm{X}^{1} \mathrm{~A} \rightarrow 8^{1} \mathrm{~A}$ & 454 & 0.0703 & $\begin{array}{l}\text { HOMO-10 } \rightarrow \text { LUMO }(83 \%) \\
\text { HOMO-7 } \rightarrow \text { LUMO }(6 \%)\end{array}$ \\
\hline & $\mathrm{S}_{20}$ & $\mathrm{X}^{1} \mathrm{~A} \rightarrow 10^{1} \mathrm{~B}$ & 429 & 0.1344 & $\begin{array}{l}\text { HOMO-4 } \rightarrow \text { LUMO+1 }(80 \%) \\
\text { HOMO-5 } \rightarrow \text { LUMO+2 }(7 \%) \\
\text { HOMO-5 } \rightarrow \text { LUMO }(7 \%)\end{array}$ \\
\hline \multirow[t]{6}{*}{$\begin{array}{l}\mathrm{n}=4 \\
\left(C_{l} \text { symmetry }\right. \\
\text { point group })\end{array}$} & $\mathrm{S}_{1}$ & $\mathrm{X}^{1} \mathrm{~A} \rightarrow 1^{1} \mathrm{~A}$ & 805 & 0.0008 & $\begin{array}{l}\text { HOMO } \rightarrow \text { LUMO }(80 \%) \\
\text { HOMO-2 } \rightarrow \text { LUMO }(10 \%) \\
\text { HOMO-1 } \rightarrow \text { LUMO+1 }(7 \%)\end{array}$ \\
\hline & $\mathrm{S}_{2}$ & $\mathrm{X}^{1} \mathrm{~A} \rightarrow 2^{1} \mathrm{~A}$ & 759 & 0.0001 & $\begin{array}{l}\text { HOMO-1 } \rightarrow \text { LUMO }(77 \%) \\
\text { HOMO } \rightarrow \text { LUMO+1 }(20 \%)\end{array}$ \\
\hline & $\mathrm{S}_{3}$ & $\mathrm{X}^{1} \mathrm{~A} \rightarrow 3^{1} \mathrm{~A}$ & 721 & 0.0037 & $\begin{array}{l}\text { HOMO-2 } \rightarrow \text { LUMO }(84 \%) \\
\text { HOMO-1 } \rightarrow \text { LUMO }+1(9 \%)\end{array}$ \\
\hline & $\mathrm{S}_{4}$ & $\mathrm{X}^{1} \mathrm{~A} \rightarrow 4^{1} \mathrm{~A}$ & 641 & 0.0188 & $\begin{array}{l}\mathrm{HOMO} \rightarrow \text { LUMO+1 }(63 \%) \\
\text { HOMO-2 } \rightarrow \text { LUMO+1 }(17 \%) \\
\text { HOMO-1 } \rightarrow \text { LUMO }(15 \%)\end{array}$ \\
\hline & $\mathrm{S}_{5}$ & $\mathrm{X}^{1} \mathrm{~A} \rightarrow 5^{1} \mathrm{~A}$ & 625 & 0.0026 & $\begin{array}{l}\text { HOMO-1 } \rightarrow \text { LUMO+1 }(72 \%) \\
\text { HOMO } \rightarrow \text { LUMO }(11 \%) \\
\text { HOMO } \rightarrow \text { LUMO }+2(8 \%)\end{array}$ \\
\hline & $\mathrm{S}_{7}$ & $\mathrm{X}^{1} \mathrm{~A} \rightarrow 7^{1} \mathrm{~A}$ & 553 & 0.0095 & $\begin{array}{l}\mathrm{HOMO} \rightarrow \mathrm{LUMO}+2(75 \%) \\
\mathrm{HOMO}-1 \rightarrow \mathrm{LUMO}+3(11 \%) \\
\mathrm{HOMO}-1 \rightarrow \text { LUMO+1 }(8 \%)\end{array}$ \\
\hline
\end{tabular}




\begin{tabular}{|c|c|c|c|c|c|}
\hline & $\mathrm{S}_{8}$ & $\mathrm{X}^{1} \mathrm{~A} \rightarrow 8^{1} \mathrm{~A}$ & 551 & 1.2660 & $\begin{array}{l}\text { HOMO-3 } \rightarrow \text { LUMO }(83 \%) \\
\text { HOMO-4 } \rightarrow \text { LUMO+1 }(9 \%)\end{array}$ \\
\hline & $\mathrm{S}_{9}$ & $\mathrm{X}^{1} \mathrm{~A} \rightarrow 9^{1} \mathrm{~A}$ & 548 & 0.0381 & $\begin{array}{l}\text { HOMO- } \rightarrow \text { LUMO+2 (66\%) } \\
\text { HOMO } \rightarrow \text { LUMO+3 }(15 \%) \\
\text { HOMO-2 } \rightarrow \text { LUMO+1 }(8 \%)\end{array}$ \\
\hline & $\mathrm{S}_{11}$ & $\mathrm{X}^{1} \mathrm{~A} \rightarrow 11^{1} \mathrm{~A}$ & 535 & 0.0108 & $\begin{array}{l}\text { HOMO-5 } \rightarrow \text { LUMO }(62 \%) \\
\text { HOMO-7 } \rightarrow \text { LUMO }(26 \%)\end{array}$ \\
\hline & $\mathrm{S}_{12}$ & $\mathrm{X}^{1} \mathrm{~A} \rightarrow 12^{1} \mathrm{~A}$ & 527 & 0.0066 & $\mathrm{HOMO}-2 \rightarrow \mathrm{LUMO}+2(90 \%)$ \\
\hline & $\mathrm{S}_{14}$ & $\mathrm{X}^{1} \mathrm{~A} \rightarrow 14^{1} \mathrm{~A}$ & 501 & 0.0237 & $\begin{array}{l}\text { HOMO-8 } \rightarrow \text { LUMO }(68 \%) \\
\text { HOMO-9 } \rightarrow \text { LUMO }+1(22 \%)\end{array}$ \\
\hline & $\mathrm{S}_{16}$ & $\mathrm{X}^{1} \mathrm{~A} \rightarrow 16^{1} \mathrm{~A}$ & 500 & 0.1045 & $\begin{array}{l}\text { HOMO-7 } \rightarrow \text { LUMO }(49 \%) \\
\text { HOMO-5 } \rightarrow \text { LUMO }(19 \%) \\
\text { HOMO-6 } \rightarrow \text { LUMO+1 }(16 \%)\end{array}$ \\
\hline & $\mathrm{S}_{18}$ & $\mathrm{X}^{1} \mathrm{~A} \rightarrow 18^{1} \mathrm{~A}$ & 492 & 0.0014 & $\begin{array}{l}\mathrm{HOMO} \rightarrow \mathrm{LUMO}+3(65 \%) \\
\mathrm{HOMO}-1 \rightarrow \mathrm{LUMO}+2(13 \%) \\
\mathrm{HOMO}-2 \rightarrow \mathrm{LUMO}+3(11 \%)\end{array}$ \\
\hline & $\mathrm{S}_{19}$ & $\mathrm{X}^{1} \mathrm{~A} \rightarrow 19^{1} \mathrm{~A}$ & 478 & 0.0111 & $\begin{array}{l}\mathrm{HOMO}-1 \rightarrow \text { LUMO+3 }(81 \%) \\
\mathrm{HOMO} \rightarrow \text { LUMO }+2(10 \%)\end{array}$ \\
\hline \multirow[t]{9}{*}{$\begin{array}{l}\mathrm{n}=5 \\
\left(D_{2} \text { symmetry }\right. \\
\text { point group) }\end{array}$} & $\mathrm{S}_{1}$ & $\mathrm{X}^{1} \mathrm{~A} \rightarrow 1^{1} \mathrm{~B}_{1}$ & 818 & 0 & $\begin{array}{l}\text { HOMO } \rightarrow \text { LUMO }(68 \%) \\
\text { HOMO-2 } \rightarrow \text { LUMO }(15 \%) \\
\text { HOMO- } \rightarrow \text { LUMO+1 }(9 \%)\end{array}$ \\
\hline & $\mathrm{S}_{2}$ & $\mathrm{X}^{1} \mathrm{~A} \rightarrow 1^{1} \mathrm{~B}_{2}$ & 784 & 0.0001 & $\begin{array}{l}\text { HOMO- } \rightarrow \text { LUMO }(52 \%) \\
\text { HOMO } \rightarrow \text { LUMO+1 }(28 \%) \\
\text { HOMO-3 } \rightarrow \text { LUMO }(15 \%)\end{array}$ \\
\hline & $\mathrm{S}_{5}$ & $\mathrm{X}^{1} \mathrm{~A} \rightarrow 3^{1} \mathrm{~B}_{2}$ & 661 & 0.0208 & $\begin{array}{l}\text { HOMO } \rightarrow \text { LUMO+1 }(53 \%) \\
\text { HOMO-1 } \rightarrow \text { LUMO }(21 \%) \\
\text { HOMO-2 } \rightarrow \text { LUMO+1 }(16 \%)\end{array}$ \\
\hline & $\mathrm{S}_{10}$ & $\mathrm{X}^{1} \mathrm{~A} \rightarrow 5^{1} \mathrm{~B}_{2}$ & 582 & 0.0374 & $\begin{array}{l}\text { HOMO-1 } \rightarrow \text { LUMO+2 (62\%) } \\
\text { HOMO } \rightarrow \text { LUMO+3 }(11 \%) \\
\text { HOMO-2 } \rightarrow \text { LUMO+1 (9\%) } \\
\text { HOMO } \rightarrow \text { LUMO+1 }(9 \%)\end{array}$ \\
\hline & $\mathrm{S}_{12}$ & $\mathrm{X}^{1} \mathrm{~A} \rightarrow 6^{1} \mathrm{~B}_{2}$ & 568 & 0.0051 & $\mathrm{HOMO}-3 \rightarrow \mathrm{LUMO}+2(86 \%)$ \\
\hline & $\mathrm{S}_{13}$ & $\mathrm{X}^{1} \mathrm{~A} \rightarrow 1^{1} \mathrm{~B}_{3}$ & 560 & 1.7869 & $\begin{array}{l}\text { HOMO-4 } \rightarrow \text { LUMO }(73 \%) \\
\text { HOMO-5 } \rightarrow \text { LUMO+1 }(9 \%) \\
\text { HOMO-6 } \rightarrow \text { LUMO }(9 \%)\end{array}$ \\
\hline & $\mathrm{S}_{16}$ & $\mathrm{X}^{1} \mathrm{~A} \rightarrow 2^{1} \mathrm{~B}_{3}$ & 537 & 0.0480 & $\begin{array}{l}\text { HOMO-6 } \rightarrow \text { LUMO (61\%) } \\
\text { HOMO-5 } \rightarrow \text { LUMO+1 (14\%) } \\
\text { HOMO-7 } \rightarrow \text { LUMO+1 (11\%) }\end{array}$ \\
\hline & $\mathrm{S}_{17}$ & $\mathrm{X}^{1} \mathrm{~A} \rightarrow 7^{1} \mathrm{~B}_{2}$ & 529 & 0.0098 & $\begin{array}{l}\mathrm{HOMO} \rightarrow \mathrm{LUMO}+3(60 \%) \\
\mathrm{HOMO}-1 \rightarrow \mathrm{LUMO}+4(16 \%) \\
\mathrm{HOMO}-1 \rightarrow \mathrm{LUMO}+2(14 \%)\end{array}$ \\
\hline & $\mathrm{S}_{19}$ & $\mathrm{X}^{1} \mathrm{~A} \rightarrow 8^{1} \mathrm{~B}_{2}$ & 511 & 0.0270 & $\begin{array}{l}\text { HOMO-2 } \rightarrow \text { LUMO+3 }(72 \%) \\
\text { HOMO } \rightarrow \text { LUMO+3 }(7 \%) \\
\text { HOMO-3 } \rightarrow \text { LUMO+4 }(5 \%)\end{array}$ \\
\hline
\end{tabular}


Table S3. The optimized Cartesian coordinates of the tetrathia[8]circulene (TTC) in the ground singlet state calculated at the B3LYP/6-31(d) level of theory

\begin{tabular}{|c|c|c|c|c|}
\hline \multirow{2}{*}{$\begin{array}{l}\text { Center } \\
\text { Number }\end{array}$} & \multirow{2}{*}{$\begin{array}{l}\text { Atomic } \\
\text { Number }\end{array}$} & \multicolumn{3}{|c|}{ Coordinates (Angstroms) } \\
\hline & & $\mathrm{X}$ & $\mathrm{Y}$ & $\mathrm{Z}$ \\
\hline 1 & 6 & -2.822081 & 3.319165 & 0.002767 \\
\hline 2 & 6 & 2.822081 & -3.319165 & 0.002767 \\
\hline 3 & 6 & -3.319165 & -2.822081 & -0.002767 \\
\hline 4 & 6 & 3.319165 & 2.822081 & -0.002767 \\
\hline 5 & 6 & 3.711728 & -2.281243 & -0.002767 \\
\hline 6 & 6 & -3.711728 & 2.281243 & -0.002767 \\
\hline 7 & 6 & -2.281243 & -3.711728 & 0.002767 \\
\hline 8 & 6 & 2.281243 & 3.711728 & 0.002767 \\
\hline 9 & 6 & -1.703121 & -0.874946 & -0.001869 \\
\hline 10 & 6 & 1.703121 & 0.874946 & -0.001869 \\
\hline 11 & 6 & 0.874946 & -1.703121 & 0.001869 \\
\hline 12 & 6 & -0.874946 & 1.703121 & 0.001869 \\
\hline 13 & 6 & -0.604176 & -1.816900 & 0.001869 \\
\hline 14 & 6 & 0.604176 & 1.816900 & 0.001869 \\
\hline 15 & 6 & -1.816900 & 0.604176 & -0.001869 \\
\hline 16 & 6 & 1.816900 & -0.604176 & -0.001869 \\
\hline 17 & 6 & -3.006375 & -1.459836 & -0.004716 \\
\hline 18 & 6 & 3.006375 & 1.459836 & -0.004716 \\
\hline 19 & 6 & 1.459836 & -3.006375 & 0.004716 \\
\hline 20 & 6 & -1.459836 & 3.006375 & 0.004716 \\
\hline 21 & 6 & -0.982863 & -3.194275 & 0.004716 \\
\hline 22 & 6 & 0.982863 & 3.194275 & 0.004716 \\
\hline 23 & 6 & -3.194275 & 0.982863 & -0.004716 \\
\hline 24 & 6 & 3.194275 & -0.982863 & -0.004716 \\
\hline 25 & 16 & -0.332423 & 4.321495 & 0.009293 \\
\hline 26 & 16 & 0.332423 & -4.321495 & 0.009293 \\
\hline 27 & 16 & -4.321495 & -0.332423 & -0.009293 \\
\hline 28 & 16 & 4.321495 & 0.332423 & -0.009293 \\
\hline 29 & 1 & 2.444215 & 4.784938 & 0.005381 \\
\hline 30 & 1 & 4.354824 & 3.147274 & -0.005381 \\
\hline 31 & 1 & 4.784938 & -2.444215 & -0.005381 \\
\hline 32 & 1 & 3.147274 & -4.354824 & 0.005381 \\
\hline 33 & 1 & -2.444215 & -4.784938 & 0.005381 \\
\hline 34 & 1 & -4.354824 & -3.147274 & -0.005381 \\
\hline 35 & 1 & -4.784938 & 2.444215 & -0.005381 \\
\hline 36 & 1 & -3.147274 & 4.354824 & 0.005381 \\
\hline
\end{tabular}

Table S4. The optimized Cartesian coordinates of the directly fused TTC-based ribbon with $\mathrm{n}=2$ (type $\mathbf{I}$ ) in the ground singlet state calculated at the B3LYP/6-31(d) level of theory

\begin{tabular}{|c|c|c|c|c|}
\hline \multirow{2}{*}{$\begin{array}{l}\text { Center } \\
\text { Number }\end{array}$} & \multirow{2}{*}{$\begin{array}{l}\text { Atomic } \\
\text { Number }\end{array}$} & \multicolumn{3}{|c|}{ Coordinates (Angstroms) } \\
\hline & & $\mathrm{X}$ & $\mathrm{Y}$ & $\mathrm{Z}$ \\
\hline 1 & 6 & -4.998721 & 1. 486051 & -0.933322 \\
\hline 2 & 6 & 6.047209 & 0.573384 & 0.436926 \\
\hline 3 & 6 & 4.998560 & 1.485709 & 0.933864 \\
\hline 4 & 6 & -6.047339 & 0.573611 & -0.436450 \\
\hline 5 & 6 & 4.998494 & -1.485702 & -0.934115 \\
\hline 6 & 6 & -6.047273 & -0.573956 & 0.436638 \\
\hline 7 & 6 & -4.998482 & -1.486192 & 0.933562 \\
\hline 8 & 6 & 6.047233 & -0.573633 & -0.436854 \\
\hline 9 & 6 & -3.573408 & -1.596938 & 0.718858 \\
\hline 10 & 6 & 2.525832 & -0.699347 & -0.200681 \\
\hline 11 & 6 & 3.573531 & -1.596799 & -0.718974 \\
\hline 12 & 6 & -2.525706 & -0.699363 & 0.200652 \\
\hline 13 & 6 & 3.573659 & 1.597099 & 0.718423 \\
\hline 14 & 6 & -2.525792 & 0.699609 & -0.200601 \\
\hline 15 & 6 & -3.573666 & 1.597075 & -0.71858 \\
\hline 16 & 6 & 2.525859 & 0.699752 & 0.200164 \\
\hline 17 & 6 & -5.585975 & -2.532656 & 1.703056 \\
\hline 18 & 6 & 1.259957 & 1.334642 & 0.211393 \\
\hline 19 & 6 & 5.585973 & -2.531824 & -1.704048 \\
\hline
\end{tabular}




\begin{tabular}{|c|c|c|}
\hline-1.259815 & 1.334449 & -0.212269 \\
\hline 5.586062 & 2.531666 & 1.704020 \\
\hline-1.259668 & -1.334012 & 0.212290 \\
\hline-5.586392 & 2.532419 & -1.702816 \\
\hline 1.259892 & -1.334129 & -0.211924 \\
\hline-2.987638 & 2.819691 & -1.151519 \\
\hline 7.330964 & -1.016672 & -0.871410 \\
\hline 2.987657 & 2.819789 & 1.151127 \\
\hline-7.331008 & -1.017394 & 0.870806 \\
\hline 2.987379 & -2.819418 & -1.151736 \\
\hline-7.331148 & 1.016799 & -0.870694 \\
\hline-2.987114 & -2.819401 & 1.151918 \\
\hline 7.330942 & 1.016261 & 0.871682 \\
\hline 4.937919 & -3.673225 & -2.189545 \\
\hline-4.937852 & -3.673956 & 2.188677 \\
\hline 0.000078 & 0.697931 & -0.000321 \\
\hline-4.938487 & 3.673881 & -2.188344 \\
\hline 4.938118 & 3.673167 & 2.189418 \\
\hline 0.000102 & -0.697392 & 0.000066 \\
\hline-3.619398 & 3.844746 & -1.863763 \\
\hline 3.619303 & 3.844529 & 1.863932 \\
\hline 8.566486 & -0.505759 & -0.462273 \\
\hline-8.566587 & -0.506266 & 0.462108 \\
\hline 3.618981 & -3.844287 & -1.864395 \\
\hline-3.618694 & -3.844525 & 1.864223 \\
\hline 8.566463 & 0.505185 & 0.462758 \\
\hline-8.566650 & 0.505437 & -0.462070 \\
\hline-7.303894 & 2.395930 & -1.926545 \\
\hline 7.303479 & 2.394781 & 1.928366 \\
\hline 7.303480 & -2.395290 & -1.927988 \\
\hline-7.303534 & -2.396580 & 1.926592 \\
\hline-1.296461 & -2.965273 & 0.819206 \\
\hline 1.296839 & -2.965584 & -0.818423 \\
\hline 1.297177 & 2.966235 & 0.817568 \\
\hline-1.297004 & 2.965857 & -0.818785 \\
\hline-3.064304 & 4.727208 & -2.166197 \\
\hline-3.063427 & -4.726843 & 2.166760 \\
\hline 3.063888 & -4.726783 & -2.166733 \\
\hline 5.486660 & -4.415045 & -2.761208 \\
\hline 5.486864 & 4.414826 & 2.761287 \\
\hline 3.064315 & 4.727123 & 2.166177 \\
\hline-5.487438 & 4.415826 & -2.759645 \\
\hline-5.486676 & -4.416014 & 2.759953 \\
\hline-9.489979 & 0.928734 & -0.844984 \\
\hline-9.489859 & -0.929743 & 0.844957 \\
\hline 9.489779 & 0.928150 & 0.846067 \\
\hline 9.489815 & -0.928860 & -0.845396 \\
\hline
\end{tabular}

Table S5. The optimized Cartesian coordinates of the directly fused TTC-based ribbon with $\mathrm{n}=3$ (type $\mathbf{I}$ ) in the ground singlet state calculated at the B3LYP/6-31(d) level of theory

\begin{tabular}{|c|c|c|c|c|}
\hline \multirow{2}{*}{$\begin{array}{l}\text { Center } \\
\text { Number }\end{array}$} & \multirow{2}{*}{$\begin{array}{l}\text { Atomic } \\
\text { Number }\end{array}$} & \multicolumn{3}{|c|}{ Coordinates (Angstroms) } \\
\hline & & $\mathrm{x}$ & $\mathrm{Y}$ & $\mathrm{Z}$ \\
\hline 1 & 6 & 0.711862 & 1.731540 & -7.626656 \\
\hline 2 & 6 & 0.789719 & 1.739663 & 9.069606 \\
\hline 3 & 6 & -1.070628 & 0.722692 & -1.748682 \\
\hline 4 & 6 & -1.478948 & 1.694391 & -0.721260 \\
\hline 5 & 6 & 0.342734 & 0.725765 & -6.606348 \\
\hline 6 & 6 & 0.607929 & 0.719699 & 10.120248 \\
\hline 7 & 6 & -1.478949 & -1.694392 & -0.721260 \\
\hline 8 & 6 & 0.342734 & -0.725765 & -6.606348 \\
\hline 9 & 6 & 0.607929 & -0.719698 & 10.120248 \\
\hline 10 & 6 & 0.711862 & -1.731540 & -7.626656 \\
\hline 11 & 6 & 0.789719 & -1.739663 & 9.069607 \\
\hline 12 & 6 & -1.070629 & -0.722693 & -1.748681 \\
\hline 13 & 6 & 0.789719 & -1.739663 & -9.069607 \\
\hline 14 & 6 & 0.711862 & -1.731540 & 7.626656 \\
\hline 15 & 6 & -1.070629 & -0.722693 & 1.748681 \\
\hline 16 & 6 & -1.478949 & -1.694392 & 0.721260 \\
\hline 17 & 6 & 0.607929 & -0.719698 & -10.120248 \\
\hline
\end{tabular}


Table S6. The optimized Cartesian coordinates of the directly fused TTC-based ribbon with $\mathrm{n}=4$ (type $\mathbf{I}$ ) in the ground singlet state calculated at the B3LYP/6-31(d) level of theory

\begin{tabular}{|c|c|c|c|c|}
\hline \multirow{2}{*}{$\begin{array}{l}\text { Center } \\
\text { Number }\end{array}$} & \multirow{2}{*}{$\begin{array}{l}\text { Atomic } \\
\text { Number }\end{array}$} & \multicolumn{3}{|c|}{ Coordinates (Angstroms) } \\
\hline & & $\mathrm{x}$ & $\mathrm{Y}$ & $\mathrm{Z}$ \\
\hline 1 & 6 & 1.240201 & 3.402509 & 1.699228 \\
\hline 2 & 6 & 0.484450 & -13.304316 & 1.739361 \\
\hline 3 & 6 & -0.243449 & 14.343136 & 0.719651 \\
\hline 4 & 6 & -0.791505 & -2.396373 & 0.722901 \\
\hline 5 & 6 & -0.484450 & 13.304316 & 1.739361 \\
\hline 6 & 6 & -1.240201 & -3.402509 & 1.699228 \\
\hline 7 & 6 & 0.791505 & 2.396373 & 0.722901 \\
\hline 8 & 6 & 0.243449 & -14.343136 & 0.719651 \\
\hline 9 & 6 & -0.484450 & 13.304316 & -1.739361 \\
\hline 10 & 6 & -1.240201 & -3.402509 & -1.699228 \\
\hline 11 & 6 & 0.791505 & 2.396373 & -0.722901 \\
\hline 12 & 6 & 0.243449 & -14.343136 & -0.719651 \\
\hline 13 & 6 & 1.240201 & 3.402509 & -1.699228 \\
\hline 14 & 6 & 0.484450 & -13.304316 & -1.739361 \\
\hline 15 & 6 & -0.243449 & 14.343136 & -0.719651 \\
\hline 16 & 6 & -0.791505 & -2.396373 & -0.722901 \\
\hline 17 & 6 & 1.317466 & 4.843194 & -1.697788 \\
\hline 18 & 6 & 0.487369 & -11.859295 & -1.731125 \\
\hline 19 & 6 & -0.174342 & 10.820008 & -0.725748 \\
\hline 20 & 6 & -0.970965 & -5.891121 & -0.722972 \\
\hline 21 & 6 & -0.487369 & 11.859295 & -1.731125 \\
\hline 22 & 6 & -1.317466 & -4.843194 & -1.697788 \\
\hline 23 & 6 & 0.970965 & 5.891121 & -0.722972 \\
\hline 24 & 6 & 0.174342 & -10.820008 & -0.725748 \\
\hline 25 & 6 & -0.487369 & 11.859295 & 1.731125 \\
\hline 26 & 6 & -1.317466 & -4.843194 & 1.697788 \\
\hline 27 & 6 & 0.970965 & 5.891121 & 0.722972 \\
\hline 28 & 6 & 0.174342 & -10.820008 & 0.725748 \\
\hline 29 & 6 & 1.317466 & 4.843194 & 1.697788 \\
\hline 30 & 6 & 0.487369 & -11.859295 & 1.731125 \\
\hline 31 & 6 & -0.174342 & 10.820008 & 0.725748 \\
\hline 32 & 6 & -0.970965 & -5.891121 & 0.722972 \\
\hline 33 & 6 & 1.506285 & 2.773709 & -2.945071 \\
\hline 34 & 6 & 0.662791 & -13.913808 & -3.016421 \\
\hline 35 & 6 & 0.004771 & 9.556719 & 1.347494 \\
\hline 36 & 6 & -0.831011 & -7.151511 & 1. 352313 \\
\hline 37 & 6 & -0.662791 & 13.913808 & -3.016421 \\
\hline 38 & 6 & -1.506285 & -2.773709 & -2.945071 \\
\hline 39 & 6 & 0.831011 & 7.151511 & 1.352313 \\
\hline 40 & 6 & -0.004771 & -9.556719 & 1.347494 \\
\hline 41 & 6 & -0.662791 & 13.913808 & 3.016421 \\
\hline 42 & 6 & -1.506285 & -2.773709 & 2.945071 \\
\hline 43 & 6 & 0.831011 & 7.151511 & -1.352313 \\
\hline 44 & 6 & -0.004771 & -9.556719 & -1.347494 \\
\hline 45 & 6 & 1.506285 & 2.773709 & 2.945071 \\
\hline 46 & 6 & 0.662791 & -13.913808 & 3.016421 \\
\hline 47 & 6 & 0.004771 & 9.556719 & -1.347494 \\
\hline 48 & 6 & -0.831011 & -7.151511 & -1.352313 \\
\hline 49 & 6 & 1.654645 & 5.441090 & 2.940921 \\
\hline 50 & 6 & 0.717818 & -11.246875 & 2.993650 \\
\hline 51 & 6 & -0.101738 & 15.619823 & -1.337765 \\
\hline 52 & 6 & -0.522757 & -1.157255 & -1.351916 \\
\hline 53 & 6 & -0.717818 & 11.246875 & 2.993650 \\
\hline 54 & 6 & -1.654645 & -5.441090 & 2.940921 \\
\hline 55 & 6 & 0.522757 & 1.157255 & -1.351916 \\
\hline 56 & 6 & 0.101738 & -15.619823 & -1.337765 \\
\hline 57 & 6 & -0.717818 & 11.246875 & -2.993650 \\
\hline 58 & 6 & -1.654645 & -5.441090 & -2.940921 \\
\hline 59 & 6 & 0.522757 & 1.157255 & 1.351916 \\
\hline 60 & 6 & 0.101738 & -15.619823 & 1.337765 \\
\hline 61 & 6 & 1.654645 & 5.441090 & -2.940921 \\
\hline 62 & 6 & 0.717818 & -11.246875 & -2.993650 \\
\hline 63 & 6 & -0.101738 & 15.619823 & 1.337765 \\
\hline 64 & 6 & -0.522757 & -1.157255 & 1.351916 \\
\hline 65 & 6 & -0.981158 & 13.260601 & -4.211540 \\
\hline 66 & 6 & -1.972798 & -3.400975 & -4.106602 \\
\hline
\end{tabular}




\begin{tabular}{|c|c|c|}
\hline 1.972798 & 3.400975 & -4.106602 \\
\hline 0.981158 & -13.260601 & -4.211540 \\
\hline 0.415406 & 8.353028 & 0.701930 \\
\hline-0.415406 & -8.353028 & 0.701930 \\
\hline 1.972798 & 3.400975 & 4.106602 \\
\hline 0.981158 & -13.260601 & 4.211540 \\
\hline-0.981158 & 13.260601 & 4.2115 \\
\hline-1.972798 & -3.400975 & 4.106602 \\
\hline 0.415406 & 8.353028 & -0.701930 \\
\hline-0.415406 & -8.353028 & -0.701930 \\
\hline 2.050238 & 4.769304 & 4.103998 \\
\hline 1.021265 & -11.892354 & 4.197226 \\
\hline-1.021265 & 11.892354 & 4.1972 \\
\hline-2.0502 & -4.769304 & 4.1039 \\
\hline 0.15097 & 16.830167 & -0.6853 \\
\hline 0.000000 & 0.000000 & -0.7029 \\
\hline-0.150971 & -16.830167 & -0.6853 \\
\hline-1.0212 & 11.892354 & -4.197226 \\
\hline-2.05023 & -4.769304 & -4.103998 \\
\hline 2.050238 & 4.769304 & -4.103998 \\
\hline 1.0212 & -11.892354 & -4.197226 \\
\hline 0.150971 & 16.830167 & 0.6853 \\
\hline 0.000000 & 0.000000 & 0.702 \\
\hline-0.150971 & -16.830167 & 0.68 \\
\hline 1.061053 & 1.099711 & 3.010 \\
\hline 0.382261 & -15.627953 & 3.051 \\
\hline-0.382261 & 15.627953 & 3.05 \\
\hline-1.06105 & -1.099711 & 3.010 \\
\hline-0.382261 & 15.627953 & -3.051 \\
\hline-1.061053 & -1.099711 & -3.010 \\
\hline 1.061053 & 1.099711 & -3.010 \\
\hline 0.382261 & -15.627953 & $-3.051 \varepsilon$ \\
\hline 1.388808 & 7.152019 & -3.006 \\
\hline 0.453531 & -9.538973 & -3.026903 \\
\hline-0.453531 & 9.538973 & -3.026903 \\
\hline-1.388808 & -7.152019 & -3.006478 \\
\hline-0.453531 & 9.538973 & 3.026903 \\
\hline-1.388808 & -7.152019 & 3.006478 \\
\hline 1.388808 & 7.152019 & 3.006478 \\
\hline 0.453531 & -9.538973 & 3.026903 \\
\hline 2.338239 & 5.326104 & 4.990268 \\
\hline 1.216397 & -11.315513 & 5.095912 \\
\hline 2.338239 & 5.326104 & -4.990268 \\
\hline 1.216397 & -11.315513 & -5.095912 \\
\hline-1.216397 & 11.315513 & -5.095912 \\
\hline-2.338239 & -5.326104 & -4.990268 \\
\hline-1.145708 & 13.828401 & -5.121966 \\
\hline-2.196684 & -2.818507 & -4.994878 \\
\hline-1.145708 & 13.828401 & 5.121966 \\
\hline-2.196684 & -2.818507 & 4.994878 \\
\hline-1.216397 & 11.315513 & 5.095912 \\
\hline-2.338239 & -5.326104 & 4.990268 \\
\hline 2.196684 & 2.818507 & 4.994878 \\
\hline 1.145708 & -13.828401 & 5.121966 \\
\hline 2.196684 & 2.818507 & -4.994878 \\
\hline 1.145708 & -13.828401 & -5.121966 \\
\hline-0.286881 & -17.743930 & 1.255498 \\
\hline-0.286881 & -17.743930 & -1.255498 \\
\hline 0.286881 & 17.743930 & 1.255498 \\
\hline 0.286881 & 17.743930 & -1.255498 \\
\hline
\end{tabular}

Table S7. The optimized Cartesian coordinates of the directly fused TTC-based ribbon with $\mathrm{n}=5$ (type $\mathbf{I}$ ) in the ground singlet state calculated at the B3LYP/6-31(d) level of theory

\begin{tabular}{|c|c|c|c|c|}
\hline \multirow{2}{*}{$\begin{array}{l}\text { Center } \\
\text { Number }\end{array}$} & \multirow{2}{*}{$\begin{array}{l}\text { Atomic } \\
\text { Number }\end{array}$} & \multicolumn{3}{|c|}{ Coordinates (Angstroms) } \\
\hline & & $\mathrm{X}$ & $\mathrm{Y}$ & $\mathrm{Z}$ \\
\hline 1 & 6 & -16.002580 & 1.731110 & -0.881047 \\
\hline 2 & 6 & 0.721493 & 1.702609 & -0.789603 \\
\hline 3 & 6 & 17.445612 & 1.739352 & -0.956291 \\
\hline 4 & 6 & -10.123428 & 0.722955 & 0.902190 \\
\hline
\end{tabular}




\begin{tabular}{|c|c|c|}
\hline 6.624095 & 0.722895 & 0.916276 \\
\hline-9.096319 & 1.697519 & 1.306694 \\
\hline 7.653579 & 1.698857 & 1.309433 \\
\hline 14.981884 & 0.725740 & -0.511938 \\
\hline 1.750654 & 0.723185 & -0.401812 \\
\hline 18.495933 & 0.719643 & -0.771702 \\
\hline-9.096319 & -1.697519 & 1.306694 \\
\hline 7.653579 & -1.698857 & 1.309433 \\
\hline 14.981884 & -0.725740 & -0.511938 \\
\hline 1.750654 & -0.723185 & -0.401812 \\
\hline 18.495933 & -0.719643 & -0.771702 \\
\hline 16.002580 & -1.731110 & -0.881047 \\
\hline 0.721493 & -1.702609 & -0.789603 \\
\hline 17.445612 & -1.739352 & -0.956291 \\
\hline 10.123428 & -0.722955 & 0.902190 \\
\hline 6.624095 & -0.722895 & 0.916276 \\
\hline 17.445612 & -1.739352 & -0.956291 \\
\hline-0.721493 & -1.702609 & -0.789603 \\
\hline 16.002580 & -1.731110 & -0.881047 \\
\hline-6.624095 & -0.722895 & 0.916276 \\
\hline 10.123428 & -0.722955 & 0.902190 \\
\hline-7.653579 & -1.698857 & 1.309433 \\
\hline 9.096319 & -1.697519 & 1.306694 \\
\hline 18.495933 & -0.719643 & -0.771702 \\
\hline-1.750654 & -0.723185 & -0.401812 \\
\hline 14.981884 & -0.725740 & -0.511938 \\
\hline-7.653579 & 1.698857 & 1.309433 \\
\hline 9.096319 & 1.697519 & 1.306694 \\
\hline 18.495933 & 0.719643 & -0.771702 \\
\hline-1.750654 & 0.723185 & -0.401812 \\
\hline 14.981884 & 0.725740 & -0.511938 \\
\hline 17.445612 & 1.739352 & -0.956291 \\
\hline-0.721493 & 1.702609 & -0.789603 \\
\hline 16.002580 & 1.731110 & -0.881047 \\
\hline-6.624095 & 0.722895 & 0.916276 \\
\hline 10.123428 & 0.722955 & 0.902190 \\
\hline 15.378499 & -2.993593 & -1.078065 \\
\hline 1.335499 & -2.950053 & -1.082301 \\
\hline 18.044545 & -3.016409 & -1.167326 \\
\hline-5.372048 & 1.352015 & 0.716488 \\
\hline 11.374087 & 1.352350 & 0.692767 \\
\hline-9.711973 & -2.940515 & 1.610789 \\
\hline 7.040478 & -2.944377 & 1.610947 \\
\hline 19.778384 & 1.337776 & -0.699060 \\
\hline-3.003288 & 1.351398 & -0.200258 \\
\hline 13.730224 & 1.347472 & -0.264396 \\
\hline-9.711973 & 2.940515 & 1.610789 \\
\hline 7.040478 & 2.944377 & 1.610947 \\
\hline 19.778384 & -1.337776 & -0.699060 \\
\hline-3.003288 & -1.351398 & -0.200258 \\
\hline 13.730224 & -1.347472 & -0.264396 \\
\hline 15.378499 & 2.993593 & -1.078065 \\
\hline 1.335499 & 2.950053 & -1.082301 \\
\hline 18.044545 & 3.016409 & -1.167326 \\
\hline-5.372048 & -1.352015 & 0.716488 \\
\hline 11.374087 & -1.352350 & 0.692767 \\
\hline 18.044545 & 3.016409 & -1.167326 \\
\hline-1.335499 & 2.950053 & -1.082301 \\
\hline 15.378499 & 2.993593 & -1.078065 \\
\hline 11.374087 & -1.352350 & 0.692767 \\
\hline 5.372048 & -1.352015 & 0.716488 \\
\hline-7.040478 & 2.944377 & 1.610947 \\
\hline 9.711973 & 2.940515 & 1.610789 \\
\hline 13.730224 & -1.347472 & -0.264396 \\
\hline 3.003288 & -1.351398 & -0.200258 \\
\hline 19.778384 & -1.337776 & -0.699060 \\
\hline-7.040478 & -2.944377 & 1.610947 \\
\hline 9.711973 & -2.940515 & 1.610789 \\
\hline 13.730224 & 1.347472 & -0.264396 \\
\hline 3.003288 & 1.351398 & -0.200258 \\
\hline 19.778384 & 1.337776 & -0.699060 \\
\hline 18.044545 & -3.016409 & -1.167326 \\
\hline-1.335499 & -2.950053 & -1.082301 \\
\hline 15.378499 & -2.993593 & -1.078065 \\
\hline 11.374087 & 1.352350 & 0.692767 \\
\hline 5.372048 & 1.352015 & 0.716488 \\
\hline-9.063099 & -4.103264 & 2.043851 \\
\hline 7.692579 & -4.105697 & 2.042681 \\
\hline 16.006604 & -4.197137 & -1.416122 \\
\hline
\end{tabular}


$-4.116272-1.503011$

$-4.211483-1.450022$

$0.685375-0.512009$

$0.702882 \quad 0.257680$

$0.701940 \quad 0.211558$

$4.197137-1.416122$

$4.116272-1.503011$

$4.211483-1.450022$

$4.103264 \quad 2.043851$

$4.105697 \quad 2.042681$

$-0.685375-0.512009$

$-0.702882 \quad 0.257680$

$-0.701940 \quad 0.211558$

$4.211483-1.450022$

$4.116272-1.503011$

$4.197137-1.416122$

$4.105697 \quad 2.042681$

$4.103264 \quad 2.043851$

$-0.701940 \quad 0.211558$

$-0.702882 \quad 0.257680$

$-0.685375-0.512009$

$-4.105697 \quad 2.042681$

$-4.103264 \quad 2.043851$

$-4.211483-1.450022$

$-4.116272-1.503011$

$-4.197137-1.416122$

$0.701940 \quad 0.211558$

$0.702882 \quad 0.257680$

$0.685375-0.512009$

$3.026782-0.721409$

$3.011798 \quad-0.733824$

$3.051858-0.979677$

3.006375 1.250152

$3.010133 \quad 1.258771$

$-3.006375 \quad 1.250152$

$-3.010133 \quad 1.258771$

$-3.026782-0.721409$

$-3.011798-0.733824$

$-3.051858 \quad-0.979677$

$-3.051858-0.979677$

$-3.011798-0.733824$

$-3.026782-0.721409$

$-3.010133 \quad 1.258771$

$-3.006375 \quad 1.250152$

3.010133 1.258771

$3.006375 \quad 1.250152$

$3.051858-0.979677$

$3.011798-0.733824$

$3.026782-0.721409$

$5.121907-1.645110$

$5.005454-1.752078$

$5.095811-1.579842$

$-5.121907-1.645110$

$-5.005454-1.752078$

$-5.095811-1.579842$

$-4.993760 \quad 2.299118$

$-4.989454 \quad 2.300945$

$\begin{array}{ll}-4.989454 & 2.300945\end{array}$

$-4.993760 \quad 2.299118$

$4.989454 \quad 2.300945$

$4.993760 \quad 2.299118$

$4.993760 \quad 2.299118$

$\begin{array}{ll}4.989454 & 2.300945\end{array}$

$5.095811-1.579842$

$5.005454-1.752078$

$5.121907-1.645110$

$-5.095811-1.579842$

$-5.005454-1.752078$

$-5.121907-1.645110$

$1.255472-0.425597$

$-1.255472 \quad-0.425597$

$\begin{array}{ll}1.255472 & -0.425597\end{array}$

$\begin{array}{ll}-1.255472 & -0.425597\end{array}$ 
Table S8. The optimized Cartesian coordinates of the TTC ribbon linked via benzene-core linker with $n=2$ (type II) in the ground singlet state calculated at the B3LYP/6-31(d) level of theory

\begin{tabular}{|c|c|c|c|c|}
\hline \multirow{2}{*}{$\begin{array}{l}\text { Center } \\
\text { Number }\end{array}$} & \multirow{2}{*}{$\begin{array}{l}\text { Atomic } \\
\text { Number }\end{array}$} & \multicolumn{3}{|c|}{ Coordinates (Angstroms) } \\
\hline & & $\mathrm{X}$ & Y & Z \\
\hline 1 & 6 & -2.772856 & -4.067690 & 4.282218 \\
\hline 2 & 6 & 3.312561 & 5.323628 & 4.280977 \\
\hline 3 & 6 & -3.312561 & -5.323628 & -4.280977 \\
\hline 4 & 6 & 2.772856 & 4.067690 & -4.282218 \\
\hline 5 & 6 & -4.214038 & -8.857509 & 0.684004 \\
\hline 6 & 6 & 0.659085 & 1.027008 & 0.708936 \\
\hline 7 & 6 & -0.659085 & -1.027008 & -0.708936 \\
\hline 8 & 6 & 4.214038 & 8.857509 & -0.684004 \\
\hline 9 & 6 & -2.772856 & -4.067690 & -4.282218 \\
\hline 10 & 6 & 3.312561 & 5.323628 & -4.280977 \\
\hline 11 & 6 & -3.312561 & -5.323628 & 4.280977 \\
\hline 12 & 6 & 2.772856 & 4.067690 & 4.282218 \\
\hline 13 & 6 & -4.214038 & -8.857509 & -0.684004 \\
\hline 14 & 6 & 0.659085 & 1.027008 & -0.708936 \\
\hline 15 & 6 & -0.659085 & -1.027008 & 0.708936 \\
\hline 16 & 6 & 4.214038 & 8.857509 & 0.684004 \\
\hline 17 & 6 & -3.404383 & -6.463395 & 0.722524 \\
\hline 18 & 6 & 1.954698 & 3.221721 & 0.735289 \\
\hline 19 & 6 & -1.954698 & -3.221721 & -0.735289 \\
\hline 20 & 6 & 3.404383 & 6.463395 & -0.722524 \\
\hline 21 & 6 & -3.098327 & -5.455533 & -1.763088 \\
\hline 22 & 6 & 2.515806 & 4.128880 & -1.763646 \\
\hline 23 & 6 & -2.515806 & -4.128880 & 1.763646 \\
\hline 24 & 6 & 3.098327 & 5.455533 & 1.763088 \\
\hline 25 & 6 & -3.404383 & -6.463395 & -0.722524 \\
\hline 26 & 6 & 1.954698 & 3.221721 & -0.735289 \\
\hline 27 & 6 & -1.954698 & -3.221721 & 0.735289 \\
\hline 28 & 6 & 3.404383 & 6.463395 & 0.722524 \\
\hline 29 & 6 & -3.098327 & -5.455533 & 1.763088 \\
\hline 30 & 6 & 2.515806 & 4.128880 & 1.763646 \\
\hline 31 & 6 & -2.515806 & -4.128880 & -1.763646 \\
\hline 32 & 6 & 3.098327 & 5.455533 & -1.763088 \\
\hline 33 & 6 & -3.849107 & -7.675513 & 1.333268 \\
\hline 34 & 6 & 1. 362448 & 2.095114 & 1.347246 \\
\hline 35 & 6 & -1.362448 & -2.095114 & -1.347246 \\
\hline 36 & 6 & 3.849107 & 7.675513 & -1.333268 \\
\hline 37 & 6 & -3.425962 & -5.978710 & -3.051408 \\
\hline 38 & 6 & 2.369009 & 3.536428 & -3.054647 \\
\hline 39 & 6 & -2.369009 & -3.536428 & 3.054647 \\
\hline 40 & 6 & 3.425962 & 5.978710 & 3.051408 \\
\hline 41 & 6 & -3.849107 & -7.675513 & -1.333268 \\
\hline 42 & 6 & 1. 362448 & 2.095114 & -1.347246 \\
\hline 43 & 6 & -1.362448 & -2.095114 & 1.347246 \\
\hline 44 & 6 & 3.849107 & 7.675513 & 1.333268 \\
\hline 45 & 6 & -3.425962 & -5.978710 & 3.051408 \\
\hline 46 & 6 & 2.369009 & 3.536428 & 3.054647 \\
\hline 47 & 6 & -2.369009 & -3.536428 & -3.054647 \\
\hline 48 & 6 & 3.425962 & 5.978710 & -3.051408 \\
\hline 49 & 6 & -0.000000 & 0.000000 & -1.393824 \\
\hline 50 & 6 & -0.000000 & 0.000000 & 1.393824 \\
\hline 51 & 16 & -1.548427 & -2.016611 & 3.065786 \\
\hline 52 & 16 & 3.984946 & 7.619509 & 3.060165 \\
\hline 53 & 16 & -3.984946 & -7.619509 & -3.060165 \\
\hline 54 & 16 & 1.548427 & 2.016611 & -3.065786 \\
\hline 55 & 16 & -3.984946 & -7.619509 & 3.060165 \\
\hline 56 & 16 & 1.548427 & 2.016611 & 3.065786 \\
\hline 57 & 16 & -1.548427 & -2.016611 & -3.065786 \\
\hline 58 & 16 & 3.984946 & 7.619509 & -3.060165 \\
\hline 59 & 1 & -0.000000 & 0.000000 & -2.479440 \\
\hline 60 & 1 & 0.000000 & 0.000000 & 2.479440 \\
\hline 61 & 1 & -2.627640 & -3.504646 & 5.198907 \\
\hline 62 & 1 & 3.622149 & 5.817028 & 5.196938 \\
\hline 63 & 1 & -3.622149 & -5.817028 & 5.196938 \\
\hline 64 & 1 & 2.627640 & 3.504646 & 5.198907 \\
\hline 65 & 1 & -3.622149 & -5.817028 & -5.196938 \\
\hline 66 & 1 & 2.627640 & 3.504646 & -5.198907 \\
\hline 67 & 1 & -2.627640 & -3.504646 & -5.198907 \\
\hline 68 & 1 & 3.622149 & 5.817028 & -5.196938 \\
\hline
\end{tabular}


Table S9. The optimized Cartesian coordinates of the TTC ribbon linked via benzene-core linker with $n=3$ (type II) in the ground singlet state calculated at the B3LYP/6-31(d) level of theory

\begin{tabular}{|c|c|c|c|c|}
\hline \multirow{2}{*}{$\begin{array}{l}\text { Center } \\
\text { Number }\end{array}$} & \multirow{2}{*}{$\begin{array}{l}\text { Atomic } \\
\text { Number }\end{array}$} & \multicolumn{3}{|c|}{ Coordinates (Angstroms) } \\
\hline & & $\mathrm{X}$ & $\mathrm{Y}$ & Z \\
\hline 1 & 6 & -4.283867 & -10.196208 & -0.910743 \\
\hline 2 & 6 & -4.237870 & 0.684127 & 1.835438 \\
\hline 3 & 6 & -4.283003 & 11.560392 & -1.015548 \\
\hline 4 & 6 & 4.283003 & -11.560392 & -1.015548 \\
\hline 5 & 6 & 4.237870 & -0.684127 & 1.835438 \\
\hline 6 & 6 & 4.283867 & 10.196208 & -0.910743 \\
\hline 7 & 6 & -0.685028 & -15.195779 & -0.790867 \\
\hline 8 & 6 & -0.710096 & -4.267652 & 0.685540 \\
\hline 9 & 6 & -0.709025 & 6.638609 & 0.108489 \\
\hline 10 & 6 & 0.709025 & -6.638609 & 0.108489 \\
\hline 11 & 6 & 0.710096 & 4.267652 & 0.685540 \\
\hline 12 & 6 & 0.685028 & 15.195779 & -0.790867 \\
\hline 13 & 6 & 4.284467 & -10.198325 & -0.900935 \\
\hline 14 & 6 & 4.237870 & 0.684112 & 1.835447 \\
\hline 15 & 6 & 4.282542 & 11.558008 & -1.028480 \\
\hline 16 & 6 & -4.282542 & -11.558008 & -1.028480 \\
\hline 17 & 6 & -4.237870 & -0.684112 & 1.835447 \\
\hline 18 & 6 & -4.284467 & 10.198325 & -0.900935 \\
\hline 19 & 6 & 0.682875 & -15.196174 & -0.787510 \\
\hline 20 & 6 & 0.710094 & -4.267651 & 0.685515 \\
\hline 21 & 6 & 0.709023 & 6.638585 & 0.108427 \\
\hline 22 & 6 & -0.709023 & -6.638585 & 0.108427 \\
\hline 23 & 6 & -0.710094 & 4.267651 & 0.685515 \\
\hline 24 & 6 & -0.682875 & 15.196174 & -0.787510 \\
\hline 25 & 6 & -0.723005 & -12.668288 & -0.767593 \\
\hline 26 & 6 & -0.733336 & -1.769001 & 1.147840 \\
\hline 27 & 6 & -0.735293 & 9.133682 & -0.413717 \\
\hline 28 & 6 & 0.735293 & -9.133682 & -0.413717 \\
\hline 29 & 6 & 0.733336 & 1.769001 & 1.147840 \\
\hline 30 & 6 & 0.723005 & 12.668288 & -0.767593 \\
\hline 31 & 6 & 1.763416 & -11.616149 & -0.785106 \\
\hline 32 & 6 & 1.748474 & -0.724039 & 1.391832 \\
\hline 33 & 6 & 1.764162 & 10.172431 & -0.655632 \\
\hline 34 & 6 & -1.764162 & -10.172431 & -0.655632 \\
\hline 35 & 6 & -1.748474 & 0.724039 & 1.391832 \\
\hline 36 & 6 & -1.763416 & 11.616149 & -0.785106 \\
\hline 37 & 6 & 0.722155 & -12.668694 & -0.764666 \\
\hline 38 & 6 & 0.733336 & -1.769001 & 1.147817 \\
\hline 39 & 6 & 0.735361 & 9.133487 & -0.414625 \\
\hline 40 & 6 & -0.735361 & -9.133487 & -0.414625 \\
\hline 41 & 6 & -0.733336 & 1.769001 & 1.147817 \\
\hline 42 & 6 & -0.722155 & 12.668694 & -0.764666 \\
\hline 43 & 6 & -1.763650 & -11.615189 & -0.790834 \\
\hline 44 & 6 & -1.748475 & -0.724034 & 1.391840 \\
\hline 45 & 6 & -1.764172 & 10.173205 & -0.651889 \\
\hline 46 & 6 & 1.764172 & -10.173205 & -0.651889 \\
\hline 47 & 6 & 1.748475 & 0.724034 & 1.391840 \\
\hline 48 & 6 & 1.763650 & 11.615189 & -0.790834 \\
\hline 49 & 6 & -1.333811 & -13.958683 & -0.812452 \\
\hline 50 & 6 & -1.348158 & -3.030954 & 1.009388 \\
\hline 51 & 6 & -1.347175 & 7.876839 & -0.211541 \\
\hline 52 & 6 & 1.347175 & -7.876839 & -0.211541 \\
\hline 53 & 6 & 1.348158 & 3.030954 & 1.009388 \\
\hline 54 & 6 & 1.333811 & 13.958683 & -0.812452 \\
\hline 55 & 6 & 3.052236 & -12.216657 & -0.924435 \\
\hline 56 & 6 & 3.028563 & -1.331097 & 1.564705 \\
\hline 57 & 6 & 3.055594 & 9.564496 & -0.699796 \\
\hline 58 & 6 & -3.055594 & -9.564496 & -0.699796 \\
\hline 59 & 6 & -3.028563 & 1.331097 & 1.564705 \\
\hline 60 & 6 & -3.052236 & 12.216657 & -0.924435 \\
\hline
\end{tabular}




\begin{tabular}{|c|c|c|}
\hline 1.332440 & -13.959438 & -0.806325 \\
\hline 1.348158 & -3.030950 & 1.009346 \\
\hline 1.347165 & 7.876631 & -0.212313 \\
\hline-1.347165 & -7.876631 & -0.212313 \\
\hline 158 & 3.030950 & \\
\hline 1.332440 & 13.959438 & -0.806 \\
\hline 3.052286 & -12.214921 & -0.935 \\
\hline 8564 & -1.331090 & 1.56 \\
\hline 3.055894 & 9.565777 & -0.694 \\
\hline 5894 & -9.565777 & -0.694 \\
\hline 3.028564 & 1.331090 & 1.56 \\
\hline 3.052286 & 12.214921 & -0.93 \\
\hline 1.394745 & -5.452891 & \\
\hline 1.394746 & 5.452883 & 0.397 \\
\hline-1.394746 & -5.452883 & \\
\hline-1.39 & 5.452 & \\
\hline-3.06 & -7.862771 & -0.405 \\
\hline 3.050693 & 3.044962 & 1.328 \\
\hline-3.0 & 13.9 & -0 . \\
\hline 3.060036 & -13.949835 & -0.942 \\
\hline 3.050693 & -3.044962 & 1.328 \\
\hline 3.066605 & 7.862771 & -0.4 \\
\hline-3.060823 & -13.9 & -0 . \\
\hline-3.050690 & -3.044960 & 1.3281 \\
\hline-3.066876 & 7.863613 & -0.4023 \\
\hline 3.066876 & -7.863613 & -0.4023 \\
\hline 3.050690 & 3.044960 & 1.3281 \\
\hline 3.060823 & 13.9 & -0 . \\
\hline 2.480507 & -5.448714 & 3906 \\
\hline 2.480509 & 5.448692 & 0.3905 \\
\hline-2.480509 & -5.448692 & 0.3905 \\
\hline-2.480507 & 5.448714 & \\
\hline-5.200758 & -9.616209 & -0.9472 \\
\hline-5.146009 & 1.258113 & 1.99074 \\
\hline-5.199274 & 12.127086 & -1.14811 \\
\hline-5.198647 & -12.124096 & -1.1647 \\
\hline-5.146004 & -1.258103 & 1.99075 \\
\hline-5.201639 & 9.618702 & -0.93633 \\
\hline 5.199274 & -12.127086 & -1.14811 \\
\hline 5.146009 & -1.258113 & 1.99074 \\
\hline 5.200758 & 9.616209 & -0.94726 \\
\hline 5.201639 & -9.618702 & -0.93633 \\
\hline 5.146004 & 1.258103 & 1.99075 \\
\hline 5.198647 & 12.124096 & -1.16474 \\
\hline-1.256424 & 16.117781 & -0.79811 \\
\hline 1.259048 & 16.117059 & -0.80426 \\
\hline-1.259048 & -16.117059 & -0.80426 \\
\hline 1.256424 & -16.117781 & -0.79811 \\
\hline
\end{tabular}

Table S10. The optimized Cartesian coordinates of the TTC ribbon linked via benzene-core linker with $n=4$ (type II) in the ground singlet state calculated at the B3LYP/6-31(d) level of theory

\begin{tabular}{|c|c|c|c|c|}
\hline \multirow{2}{*}{$\begin{array}{l}\text { Center } \\
\text { Number }\end{array}$} & \multirow{2}{*}{$\begin{array}{l}\text { Atomic } \\
\text { Number }\end{array}$} & \multicolumn{3}{|c|}{ Coordinates (Angstroms) } \\
\hline & & $\mathrm{X}$ & $\mathrm{Y}$ & $\mathrm{Z}$ \\
\hline 1 & 6 & 15.775857 & -4.281259 & -0.515320 \\
\hline 2 & 6 & 4.627086 & -4.176727 & -1.855720 \\
\hline 3 & 6 & -5.988619 & -4.176226 & 1.986703 \\
\hline 4 & 6 & -17.085090 & -4.300227 & 0.124900 \\
\hline 5 & 6 & 16.990690 & 4.222179 & 0.718172 \\
\hline 6 & 6 & 6.031271 & 4.299144 & -1.809010 \\
\hline 7 & 6 & -4.665797 & 4.302112 & 1.717758 \\
\hline 8 & 6 & -15.720122 & 4.252958 & -0.214209 \\
\hline 9 & 6 & 20.285015 & -0.845137 & 1.891885 \\
\hline 10 & 6 & 9.671477 & -0.683712 & -1.073955 \\
\hline 11 & 6 & -1.160587 & -0.654880 & 0.374535 \\
\hline 12 & 6 & -12.079361 & -0.704147 & 0.681779 \\
\hline 13 & 6 & 12.083286 & 0.712742 & -0.622947 \\
\hline 14 & 6 & 1.161436 & 0.765230 & -0.374565 \\
\hline 15 & 6 & -9.676048 & 0.735771 & 1.021010 \\
\hline 16 & 6 & -20.251704 & 0.510420 & -2.067086 \\
\hline
\end{tabular}




\begin{tabular}{|c|c|c|}
\hline 15.719011 & 4.253305 & 0.217459 \\
\hline 4.665780 & 4.300762 & -1.723228 \\
\hline-6.031212 & 4.300345 & 1.804746 \\
\hline-16.992237 & 4.221832 & -0.713797 \\
\hline 17.086432 & -4.299059 & -0.127083 \\
\hline 5.989180 & -4.177561 & -1.98463 \\
\hline-4.626451 & -4.175235 & 1.85860 \\
\hline-15.774545 & -4.282227 & 0.5132 \\
\hline 20.250276 & 0.510667 & 2.0709 \\
\hline 9.676108 & 0.735378 & -1.0214 \\
\hline-1.161421 & 0.765547 & 0.3718 \\
\hline-12.083320 & 0.712776 & 0.6230 \\
\hline 12.079565 & -0.704183 & -0.6818 \\
\hline 1.160959 & -0.655203 & -0.3749 \\
\hline-9.671210 & -0.683319 & \\
\hline-20.285746 & -0.845599 & -1.8895 \\
\hline 17.962112 & -0.812117 & 0.8963 \\
\hline 7.142411 & -0.689248 & -1.3251 \\
\hline-3.615462 & -0.678 & 1.0320 \\
\hline-14.576 & -0.759103 & 0.1730 \\
\hline 14.573113 & 0.708558 & -0.07762 \\
\hline 3.619377 & 0.787221 & -1.02053 \\
\hline-7.148220 & 0.777523 & 1.2821 \\
\hline-17.935450 & 0.622 & -1.0 \\
\hline 16.972026 & 1.692 & 0.7143 \\
\hline 6.092521 & 1.800353 & -1.4239 \\
\hline-4.647760 & 1.804371 & 1.32082 \\
\hline-15.611803 & 1.722 & -0.2 \\
\hline 15.633229 & -1.792392 & -0.0709 \\
\hline 4.633895 & -1.693977 & -1.3739 \\
\hline-6.076038 & -1.695617 & 1.5014 \\
\hline-17.017972 & -1.8162 & -0.3536 \\
\hline 17.934576 & 0.622906 & 1.0640 \\
\hline 7.148361 & 0.776815 & -1.2833 \\
\hline-3.619287 & 0.788000 & 1.0181 \\
\hline-14.573355 & 0.708 & 0.078 \\
\hline 14.576545 & -0.758794 & -0.172 \\
\hline 3.615804 & -0.679426 & -1.0325 \\
\hline-7.142118 & -0.688525 & 1.32442 \\
\hline-17.962354 & -0.812669 & -0.89504 \\
\hline 17.018318 & -1.8 & 0 . \\
\hline 6.076420 & -1.696553 & -1.50141 \\
\hline-4.633471 & -1.692953 & 1.3744 \\
\hline-15.632822 & -1.792955 & 0.07072 \\
\hline 15.611190 & 1.723310 & 0.21811 \\
\hline 4.647822 & 1.803389 & -1.3239 \\
\hline-6.092404 & 1.801242 & 1.4216 \\
\hline-16.972989 & 1.691693 & -0.71144 \\
\hline 19.176100 & -1.450135 & 1.29511 \\
\hline 8.409255 & -1.309577 & -1.3110 \\
\hline-2.367372 & -1.292483 & 0.7968 \\
\hline-13.342048 & -1.352908 & 0.51612 \\
\hline 13.344601 & 1.338097 & -0.37620 \\
\hline 2.371948 & 1.402960 & -0.78606 \\
\hline-8.419496 & 1.385585 & 1.22121 \\
\hline-19.116116 & 1.194481 & -1.62613 \\
\hline 17.556199 & 2.969654 & 0.97354 \\
\hline 6.690844 & 3.083147 & -1.60762 \\
\hline-4.034465 & 3.088515 & 1.42984 \\
\hline-15.072479 & 3.030376 & -0.01881 \\
\hline 15.097377 & -3.061765 & -0.44623 \\
\hline 4.010675 & -2.970870 & -1.50901 \\
\hline-6.660947 & -2.972725 & 1.75465 \\
\hline-17.645239 & -3.099334 & -0.32013 \\
\hline 19.114677 & 1.194746 & 1.63006 \\
\hline 8.419547 & 1.385025 & -1.22215 \\
\hline-2.372025 & 1.403641 & 0.78249 \\
\hline-13.344818 & 1.337961 & 0.37683 \\
\hline 13.342361 & -1.352745 & -0.51622 \\
\hline 2.367862 & -1.293191 & -0.79630 \\
\hline-8.408891 & -1.308994 & 1.31057 \\
\hline-19.176252 & -1.450774 & -1.29400 \\
\hline 17.646096 & -3.098337 & 0.31902 \\
\hline 6.661422 & -2.973755 & -1.75392 \\
\hline-4.010085 & -2.969599 & 1.51106 \\
\hline-15.096536 & -3.062415 & 0.44509 \\
\hline 15.071756 & 3.030695 & 0.02094 \\
\hline 4.034407 & 3.087329 & -1.43468 \\
\hline & & 1 \\
\hline
\end{tabular}




\begin{tabular}{|c|c|c|}
\hline-17.557674 & 2.969319 & -0.969381 \\
\hline 10.880206 & 1.408760 & -0.789684 \\
\hline-0.000078 & 1.450869 & -0.001927 \\
\hline-10.880309 & 1.408978 & 0.789557 \\
\hline 10.872659 & -1.379117 & -0.898455 \\
\hline 0.000276 & -1.340597 & 0.000363 \\
\hline-10.872306 & -1.378904 & 0.898132 \\
\hline 13.411079 & -3.054893 & -0.819784 \\
\hline 2.323942 & -2.993362 & -1.124798 \\
\hline-8.389073 & -3.002295 & 1.674373 \\
\hline-19.255168 & -3.149710 & -0.960900 \\
\hline 19.129603 & 2.926568 & 1.699692 \\
\hline 8.417122 & 3.095835 & -1.492557 \\
\hline-2.340636 & 3.110197 & 1.077550 \\
\hline-13.409502 & 3.065745 & 0.447436 \\
\hline 19.255864 & -3.148686 & 0.960215 \\
\hline 8.389582 & -3.003038 & -1.674142 \\
\hline-2.323215 & -2.992228 & 1.127470 \\
\hline-13.410253 & -3.055252 & 0.818677 \\
\hline 13.409042 & 3.065918 & -0.446301 \\
\hline 2.340377 & 3.109128 & -1.083344 \\
\hline-8.417109 & 3.096518 & 1.490838 \\
\hline-19.131669 & 2.926349 & -1.694243 \\
\hline 10.872766 & 2.491097 & -0.702882 \\
\hline-0.000203 & 2.536814 & -0.002783 \\
\hline-10.873075 & 2.491318 & 0.702841 \\
\hline 10.860897 & -2.464911 & -0.896826 \\
\hline 0.000418 & -2.426585 & 0.001251 \\
\hline-10.860360 & -2.464693 & 0.896459 \\
\hline 15.260813 & -5.178532 & -0.844040 \\
\hline 4.038944 & -5.081718 & -1.971346 \\
\hline-6.543483 & -5.081929 & 2.210574 \\
\hline-17.672308 & -5.213163 & 0.126099 \\
\hline 17.673998 & -5.211770 & -0.129023 \\
\hline 6.544078 & -5.083365 & -2.208027 \\
\hline-4.038195 & -5.080012 & 1.975376 \\
\hline-15.259160 & -5.179561 & 0.841228 \\
\hline 17.546755 & 5.129722 & 0.931288 \\
\hline 6.596691 & 5.209511 & -1.981716 \\
\hline-4.085448 & 5.214059 & 1.816950 \\
\hline-15.205708 & 5.186324 & -0.007757 \\
\hline 15.204585 & 5.186672 & 0.011054 \\
\hline 4.085368 & 5.212529 & -1.823692 \\
\hline-6.596637 & 5.210762 & 1.977167 \\
\hline-17.548688 & 5.129369 & -0.925932 \\
\hline-21.147187 & -1.440362 & -2.176932 \\
\hline-21.085033 & 1.053039 & -2.50246 \\
\hline 21.146563 & -1.439840 & 2.179098 \\
\hline 21.083136 & 1.053156 & 2.507408 \\
\hline
\end{tabular}

Table S11. The optimized Cartesian coordinates of the TTC ribbon linked via benzene-core linker with $n=5$ (type II) in the ground singlet state calculated at the B3LYP/6-31(d) level of theory

\begin{tabular}{|c|c|c|c|c|}
\hline \multirow{2}{*}{$\begin{array}{l}\text { Center } \\
\text { Number }\end{array}$} & \multirow{2}{*}{$\begin{array}{l}\text { Atomic } \\
\text { Number }\end{array}$} & \multicolumn{3}{|c|}{ Coordinates (Angstroms) } \\
\hline & & $\mathrm{x}$ & Y & $\mathrm{Z}$ \\
\hline 1 & 6 & 21.104712 & 4.283928 & -1.096744 \\
\hline 2 & 6 & 10.232820 & 4.238249 & 1.682070 \\
\hline 3 & 6 & -0.684081 & 4.238956 & -1.148489 \\
\hline 4 & 6 & -11.601026 & 4.238242 & 1.676503 \\
\hline 5 & 6 & -22.466379 & 4.282551 & -1.216161 \\
\hline 6 & 6 & 22.466379 & -4.282551 & -1.216161 \\
\hline 7 & 6 & 11.601026 & -4.238242 & 1.676503 \\
\hline 8 & 6 & 0.684081 & -4.238956 & -1.148489 \\
\hline 9 & 6 & -10.232820 & -4.238249 & 1.682070 \\
\hline 10 & 6 & -21.104712 & -4.283928 & -1.096744 \\
\hline 11 & 6 & 26.103862 & 0.683962 & -0.988881 \\
\hline 12 & 6 & 15.180309 & 0.710082 & 0.515242 \\
\hline 13 & 6 & 4.269681 & 0.710210 & -0.010473 \\
\hline 14 & 6 & -6.644066 & 0.710240 & 0.550124 \\
\hline 15 & 6 & -17.549292 & 0.709034 & -0.069801 \\
\hline
\end{tabular}




\begin{tabular}{|c|c|c|}
\hline 17.549292 & -0.709034 & -0.069801 \\
\hline 6.644066 & -0.710240 & 0.550124 \\
\hline-4.269681 & -0.710210 & -0.010473 \\
\hline 15.180309 & -0.710082 & 0.515242 \\
\hline 6.103862 & -0.683962 & -0.988881 \\
\hline 1.104712 & -4.283928 & -1.096744 \\
\hline 10.232820 & -4.238249 & 1.682070 \\
\hline-0.684081 & -4.238956 & -1.148489 \\
\hline 1.601026 & -4.238242 & 1.676503 \\
\hline 2.466379 & -4.282551 & -1.216161 \\
\hline 2.466379 & 4.282551 & -1.216161 \\
\hline 11.601026 & 4.238242 & 1.676503 \\
\hline 0.684081 & 4.238956 & -1.148489 \\
\hline 10.232820 & 4.238249 & 1.682070 \\
\hline 1 & 4.283928 & -1.096744 \\
\hline 6.103862 & -0.683962 & -0.988881 \\
\hline 5.180309 & -0.710082 & 0.515242 \\
\hline 4.269681 & -0.710210 & -0.010473 \\
\hline-6.644066 & -0.710240 & 0.550124 \\
\hline 17.549292 & -0.709034 & -0.069801 \\
\hline .7 .549292 & 0.709034 & -0.069801 \\
\hline 6.644066 & 0.710240 & 0.550124 \\
\hline-4.269681 & 0.710210 & -0.010473 \\
\hline 5.180309 & 0.710082 & 0.515242 \\
\hline 6.103862 & 0.683962 & -0.988881 \\
\hline 3.576451 & 0.722573 & -0.960898 \\
\hline 12.683249 & 0.733344 & 0.986487 \\
\hline 69562 & 0.733 & 6896 \\
\hline-9.144948 & 0.733308 & 1.001062 \\
\hline 20.042586 & 0.735316 & -0.600195 \\
\hline 20.042586 & -0.735316 & -0.600195 \\
\hline 9.144948 & -0.733308 & 1.001062 \\
\hline-1.76 & -0.733328 & -0.466896 \\
\hline-12.683249 & -0.733344 & 0.986487 \\
\hline-23.576451 & -0.722573 & -0.960898 \\
\hline 22.523568 & -1.763473 & -0.980863 \\
\hline 11.639162 & -1.748555 & 1.234015 \\
\hline 0.724096 & -1.748727 & -0.708427 \\
\hline-10.191065 & -1.748555 & 1.239928 \\
\hline-21.081069 & -1.764104 & -0.8 \\
\hline 21.081069 & 1.764104 & 3150 \\
\hline 10.191065 & 1.748555 & 1.239928 \\
\hline-0.724096 & 1.748727 & -0.708427 \\
\hline-11.639162 & 1.748555 & 1.234015 \\
\hline 22.523568 & 1.763473 & -0.980863 \\
\hline 23.576451 & -0.722573 & -0.960898 \\
\hline 12.683249 & -0.733344 & 0.986487 \\
\hline 1.769562 & -0.733328 & -0.466896 \\
\hline-9.144948 & -0.733308 & 1.001062 \\
\hline-20.042586 & -0.735316 & -0.600195 \\
\hline 20.042586 & 0.735316 & -0.600195 \\
\hline 9.144948 & 0.733308 & 1.001062 \\
\hline-1.769562 & 0.733328 & -0.466896 \\
\hline-12.683249 & 0.733344 & 0.986487 \\
\hline-23.576451 & 0.722573 & -0.960898 \\
\hline 22.523568 & 1.763473 & -0.980863 \\
\hline 11.639162 & 1.748555 & 1.234015 \\
\hline 0.724096 & 1.748727 & -0.708427 \\
\hline-10.191065 & 1.748555 & 1.239928 \\
\hline-21.081069 & 1.764104 & -0.843150 \\
\hline 21.081069 & -1.764104 & -0.843150 \\
\hline 10.191065 & -1.748555 & 1.239928 \\
\hline-0.724096 & -1.748727 & -0.708427 \\
\hline-11.639162 & -1.748555 & 1.234015 \\
\hline-22.523568 & -1.763473 & -0.980863 \\
\hline 24.866910 & 1.333145 & -1.006840 \\
\hline 13.944703 & 1.348138 & 0.843273 \\
\hline 3.031900 & 1. 348065 & -0.330301 \\
\hline-7.882282 & 1.348097 & 0.868276 \\
\hline-18.786339 & 1.347175 & -0.394382 \\
\hline 18.786339 & -1.347175 & -0.394382 \\
\hline 7.882282 & -1.348097 & 0.868276 \\
\hline-3.031900 & -1.348065 & -0.330301 \\
\hline-13.944703 & -1.348138 & 0.843273 \\
\hline-24.866910 & -1.333145 & -1.006840 \\
\hline 23.123304 & -3.052159 & -1.124646 \\
\hline 12.246866 & -3.028754 & 1.403920 \\
\hline 1.330957 & -3.029127 & -0.879978 \\
\hline .584792 & -3.028747 & 1.414771 \\
\hline
\end{tabular}


-20.473177
20.473177
9.584792

$-3.055599$

3. 055599

3.028747

3.029127

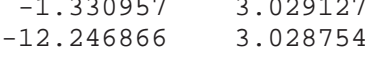

$-23.123304$

24.866910

13.944703

3. 031900

$-7.882282$

$-18.786339$

18.786339

7. 882282

$-3.031900$

$-13.944703$

$-24.866910$

23.123304

12.246866

1. 330957

$-9.584792$

$-20.473177$

20.473177

9.584792

$-1.330957$

$-12.246866$

$-23.123304$

16.364568

5.456831

$-5.456831$

$-16.364568$

16.364568

5.456831

$-5.456831$

$-16.364568$

18.772077

7.869941

$-3.045005$

$-13.959820$

$-24.856429$

24.856429

13.959820

3.045005

$-7.869941$

$-18.772077$

24.856429

13.959820

3. 045005

$-7.869941$

$-18.772077$

18.772077

7.869941

$-3.045005$

$-13.959820$

$-24.856429$

16.360430

5.456944

$-5.456944$

$-16.360430$

16.360430

5.456944

$-5.456944$

$-16.360430$

20.524765

9.659436

$-1.258162$

$-12.175671$

$-23.032378$

23.032378

12.175671

1.258162

$-9.659436$

$-20.524765$

23.032378

12.175671

1.258162
-9.659436

$-9.659436$

$-20.524765$

20.524765

9.659436

3.052159
-1.333145

$-1.348138$

$-1.348065$

$-1.348097$

$-1.347175$

1. 347175

1.348097

1. 348065

1. 348138

1. 333145

3. 052159

3. 028754

3. 029127

3. 028747

3. 055599

$-3.055599$

$-3.028747$

$-3.029127$

$-3.028754$

$-3.052159$

$-1.394750$

$-1.395748$

$-1.395748$

$-1.394750$

1.394750

1. 395748

1. 395748

1. 394750

3. 066635

3.050918

3. 051217

3.050806

3. 060398

$-3.060398$

$-3.050806$

$-3.051217$

$-3.050918$

$-3.066635$

3. 060398

3. 050806

3.051217

3.050918

3.066635

$-3.066635$

$-3.050918$

$-3.051217$

$-3.050806$

$-3.060398$

$-2.480512$

$-2.481719$

$-2.481719$

$-2.480512$

2. 480512

2. 481719

2. 481719

2. 480512

5.200906

5.146438

5.147217

5.146436

5.198709

5.198709

5.146436

5.147217

5.146438

5.200906

$-5.198709$

$-5.146436$

$-5.147217$

$-5.146438$

$-5.200906$

$-5.200906$

$-5.146438$

$-0.885523$

$-0.885523$

1. 414771

$-0.879978$

1. 403920

$-1.124646$

$-1.006840$

0.843273 
Table S12. The optimized Cartesian coordinates of the TTC ribbon linked through four-membered ring with $n=2$ (type III) in the ground singlet state calculated at the B3LYP/6-31(d) level of theory

\begin{tabular}{|c|c|c|c|c|}
\hline \multirow{2}{*}{$\begin{array}{l}\text { Center } \\
\text { Number }\end{array}$} & \multirow{2}{*}{$\begin{array}{l}\text { Atomic } \\
\text { Number }\end{array}$} & \multicolumn{3}{|c|}{ Coordinates (Angstroms) } \\
\hline & & $\mathrm{X}$ & $\mathrm{Y}$ & Z \\
\hline 1 & 6 & 4.347580 & 4.266703 & 0.527431 \\
\hline 2 & 6 & -5.700263 & 4.235249 & -0.722005 \\
\hline 3 & 6 & 5.700263 & -4.235249 & -0.722005 \\
\hline 4 & 6 & -4.347580 & -4.266703 & 0.527431 \\
\hline 5 & 6 & 9.309723 & 0.655513 & 0.193026 \\
\hline 6 & 6 & -0.753813 & 0.695769 & -0.000490 \\
\hline 7 & 6 & 0.753813 & -0.695769 & -0.000490 \\
\hline 8 & 6 & -9.309723 & -0.655513 & 0.193026 \\
\hline 9 & 6 & 4.347580 & -4.266703 & -0.527431 \\
\hline 10 & 6 & -5.700263 & -4.235249 & 0.722005 \\
\hline 11 & 6 & 5.700263 & 4.235249 & 0.722005 \\
\hline 12 & 6 & -4.347580 & 4.266703 & -0.527431 \\
\hline 13 & 6 & 9.309723 & -0.655513 & -0.193026 \\
\hline 14 & 6 & -0.753813 & -0.695769 & 0.000490 \\
\hline 15 & 6 & 0.753813 & 0.695769 & 0.000490 \\
\hline 16 & 6 & -9.309723 & 0.655513 & -0.193026 \\
\hline 17 & 6 & 6.781271 & 0.703381 & 0.165613 \\
\hline 18 & 6 & -3.234393 & 0.720482 & -0.040153 \\
\hline 19 & 6 & 3.234393 & -0.720482 & -0.040153 \\
\hline 20 & 6 & -6.781271 & -0.703381 & 0.165613 \\
\hline 21 & 6 & 5.732212 & -1.739113 & -0.317820 \\
\hline 22 & 6 & -4.289204 & -1.757986 & 0.192602 \\
\hline 23 & 6 & 4.289204 & 1.757986 & 0.192602 \\
\hline 24 & 6 & -5.732212 & 1.739113 & -0.317820 \\
\hline 25 & 6 & 6.781271 & -0.703381 & -0.165613 \\
\hline 26 & 6 & -3.234393 & -0.720482 & 0.040153 \\
\hline 27 & 6 & 3.234393 & 0.720482 & 0.040153 \\
\hline 28 & 6 & -6.781271 & 0.703381 & -0.165613 \\
\hline 29 & 6 & 5.732212 & 1.739113 & 0.317820 \\
\hline 30 & 6 & -4.289204 & 1.757986 & -0.192602 \\
\hline 31 & 6 & 4.289204 & -1.757986 & -0.192602 \\
\hline 32 & 6 & -5.732212 & -1.739113 & 0.317820 \\
\hline 33 & 6 & 8.071382 & 1.285742 & 0.350823 \\
\hline 34 & 6 & -1.937475 & 1.381392 & -0.027078 \\
\hline 35 & 6 & 1.937475 & -1.381392 & -0.027078 \\
\hline 36 & 6 & -8.071382 & -1.285742 & 0.350823 \\
\hline 37 & 6 & 6.340615 & -2.999342 & -0.604786 \\
\hline 38 & 6 & -3.696787 & -3.055325 & 0.279551 \\
\hline 39 & 6 & 3.696787 & 3.055325 & 0.279551 \\
\hline 40 & 6 & -6.340615 & 2.999342 & -0.604786 \\
\hline 41 & 6 & 8.071382 & -1.285742 & -0.350823 \\
\hline 42 & 6 & -1.937475 & -1.381392 & 0.027078 \\
\hline 43 & 6 & 1.937475 & 1.381392 & 0.027078 \\
\hline 44 & 6 & -8.071382 & 1.285742 & -0.350823 \\
\hline 45 & 6 & 6.340615 & 2.999342 & 0.604786 \\
\hline 46 & 6 & -3.696787 & 3.055325 & -0.279551 \\
\hline 47 & 6 & 3.696787 & -3.055325 & -0.279551 \\
\hline 48 & 6 & -6.340615 & -2.999342 & 0.604786 \\
\hline 49 & 16 & 1.968770 & 3.109497 & 0.112723 \\
\hline 50 & 16 & -8.067003 & 2.970468 & -0.753782 \\
\hline 51 & 16 & 8.067003 & -2.970468 & -0.753782 \\
\hline 52 & 16 & -1.968770 & -3.109497 & 0.112723 \\
\hline 53 & 16 & 8.067003 & 2.970468 & 0.753782 \\
\hline 54 & 16 & -1.968770 & 3.109497 & -0.112723 \\
\hline 55 & 16 & 1.968770 & -3.109497 & -0.112723 \\
\hline 56 & 16 & -8.067003 & -2.970468 & 0.753782 \\
\hline 57 & 1 & 3.781076 & 5.191050 & 0.582043 \\
\hline
\end{tabular}


Table S13. The optimized Cartesian coordinates of the TTC ribbon linked through four-membered ring with $n=3$ (type III) in the ground singlet state calculated at the B3LYP/6-31(d) level of theory

\begin{tabular}{|c|c|c|c|c|}
\hline \multirow{2}{*}{$\begin{array}{l}\text { Center } \\
\text { Number }\end{array}$} & \multirow{2}{*}{$\begin{array}{l}\text { Atomic } \\
\text { Number }\end{array}$} & \multicolumn{3}{|c|}{ Coordinates (Angstroms) } \\
\hline & & $\mathrm{X}$ & $\mathrm{Y}$ & $\mathrm{Z}$ \\
\hline 1 & 6 & -9.351163 & 4.264683 & 0.561432 \\
\hline 2 & 6 & 0.664959 & 4.285345 & -0.159682 \\
\hline 3 & 6 & 10.708164 & 4.279765 & -0.400233 \\
\hline 4 & 6 & -10.708164 & -4.279765 & -0.400233 \\
\hline 5 & 6 & -0.664959 & -4.285345 & -0.159682 \\
\hline 6 & 6 & 9.351163 & -4.264683 & 0.561432 \\
\hline 7 & 6 & -14.317393 & 0.683170 & -0.002763 \\
\hline 8 & 6 & -4.248575 & 0.678670 & 0.155576 \\
\hline 9 & 6 & 5.756277 & 0.677794 & -0.155888 \\
\hline 10 & 6 & -5.756277 & -0.677794 & -0.155888 \\
\hline 11 & 6 & 4.248575 & -0.678670 & 0.155576 \\
\hline 12 & 6 & 14.317393 & -0.683170 & -0.002763 \\
\hline 13 & 6 & -9.351163 & -4.264683 & -0.561432 \\
\hline 14 & 6 & 0.664959 & -4.285345 & 0.159682 \\
\hline 15 & 6 & 10.708164 & -4.279765 & 0.400233 \\
\hline 16 & 6 & -10.708164 & 4.279765 & 0.400233 \\
\hline 17 & 6 & -0.664959 & 4.285345 & 0.159682 \\
\hline 18 & 6 & 9.351163 & 4.264683 & -0.561432 \\
\hline 19 & 6 & -14.317393 & -0.683170 & 0.002763 \\
\hline 20 & 6 & -4.248575 & -0.678670 & -0.155576 \\
\hline 21 & 6 & 5.756277 & -0.677794 & 0.155888 \\
\hline 22 & 6 & -5.756277 & 0.677794 & 0.155888 \\
\hline 23 & 6 & 4.248575 & 0.678670 & -0.155576 \\
\hline 24 & 6 & 14.317393 & 0.683170 & 0.002763 \\
\hline 25 & 6 & -11.787922 & 0.722186 & 0.030136 \\
\hline 26 & 6 & -1.771625 & 0.712647 & 0.102732 \\
\hline 27 & 6 & 8.237928 & 0.709561 & -0.132373 \\
\hline 28 & 6 & -8.237928 & -0.709561 & -0.132373 \\
\hline 29 & 6 & 1.771625 & -0.712647 & 0.102732 \\
\hline 30 & 6 & 11.787922 & -0.722186 & 0.030136 \\
\hline 31 & 6 & -10.738587 & -1.762850 & -0.147137 \\
\hline 32 & 6 & -0.716995 & -1.758095 & -0.103291 \\
\hline 33 & 6 & 9.293284 & -1.751929 & 0.250060 \\
\hline 34 & 6 & -9.293284 & 1.751929 & 0.250060 \\
\hline 35 & 6 & 0.716995 & 1.758095 & -0.103291 \\
\hline 36 & 6 & 10.738587 & 1.762850 & -0.147137 \\
\hline 37 & 6 & -11.787922 & -0.722186 & -0.030136 \\
\hline 38 & 6 & -1.771625 & -0.712647 & -0.102732 \\
\hline 39 & 6 & 8.237928 & -0.709561 & 0.132373 \\
\hline 40 & 6 & -8.237928 & 0.709561 & 0.132373 \\
\hline 41 & 6 & 1.771625 & 0.712647 & -0.102732 \\
\hline 42 & 6 & 11.787922 & 0.722186 & -0.030136 \\
\hline 43 & 6 & -10.738587 & 1.762850 & 0.147137 \\
\hline 44 & 6 & -0.716995 & 1.758095 & 0.103291 \\
\hline 45 & 6 & 9.293284 & 1.751929 & -0.250060 \\
\hline 46 & 6 & -9.293284 & -1.751929 & -0.250060 \\
\hline 47 & 6 & 0.716995 & -1.758095 & 0.103291 \\
\hline 48 & 6 & 10.738587 & -1.762850 & 0.147137 \\
\hline 49 & 6 & -13.078855 & 1.332045 & 0.016751 \\
\hline 50 & 6 & -3.064694 & 1.355987 & 0.272072 \\
\hline 51 & 6 & 6.939981 & 1.349991 & -0.291575 \\
\hline 52 & 6 & -6.939981 & -1.349991 & -0.291575 \\
\hline 53 & 6 & 3.064694 & -1.355987 & 0.272072 \\
\hline
\end{tabular}




\begin{tabular}{|c|c|c|}
\hline 13.078855 & -1.332045 & 0.016751 \\
\hline-11.348756 & -3.053365 & -0.208990 \\
\hline-1.305651 & -3.048931 & -0.268341 \\
\hline 8.699441 & -3.031527 & 0.478727 \\
\hline-8.699441 & 3.031527 & 0.478727 \\
\hline 1.305651 & 3.048931 & -0.268341 \\
\hline 11.348756 & 3.053365 & -0.208990 \\
\hline-13.078855 & -1.332045 & -0.016751 \\
\hline-3.064694 & -1.355987 & -0.272072 \\
\hline 6.939981 & -1.349991 & 0.291575 \\
\hline-6.939981 & 1.349991 & 0.291575 \\
\hline 3.064694 & 1.355987 & -0.272072 \\
\hline 13.078855 & 1.332045 & -0.016751 \\
\hline-11.348756 & 3.053365 & 0.208990 \\
\hline-1.305651 & 3.048931 & 0.268341 \\
\hline 8.699441 & 3.031527 & -0.478727 \\
\hline-8.699441 & -3.031527 & -0.478727 \\
\hline 1.305651 & -3.048931 & 0.268341 \\
\hline 11.348756 & -3.053365 & 0.208990 \\
\hline-6.968755 & 3.048478 & 0.618221 \\
\hline 3.023367 & 3.066857 & -0.535374 \\
\hline 13.076689 & 3.062440 & -0.083967 \\
\hline-13.076689 & -3.062440 & -0.083967 \\
\hline-3.023367 & -3.066857 & -0.535374 \\
\hline 6.968755 & -3.048478 & 0.618221 \\
\hline-13.076689 & 3.062440 & 0.083967 \\
\hline-3.023367 & 3.066857 & 0.535374 \\
\hline 6.968755 & 3.048478 & -0.618221 \\
\hline-6.968755 & -3.048478 & -0.618221 \\
\hline 3.023367 & -3.066857 & 0.535374 \\
\hline 13.076689 & -3.062440 & 0.083967 \\
\hline-8.782633 & 5.173832 & 0.730391 \\
\hline 1.223628 & 5.206612 & -0.291247 \\
\hline 11.281691 & 5.200671 & -0.436246 \\
\hline-11.281691 & 5.200671 & 0.436246 \\
\hline-1.223628 & 5.206612 & 0.291247 \\
\hline 8.782633 & 5.173832 & -0.730391 \\
\hline-8.782633 & -5.173832 & -0.730391 \\
\hline 1.223628 & -5.206612 & 0.291247 \\
\hline 11.281691 & -5.200671 & 0.436246 \\
\hline-11.281691 & -5.200671 & -0.436246 \\
\hline-1.223628 & -5.206612 & -0.291247 \\
\hline 8.782633 & -5.173832 & 0.730391 \\
\hline 15.238129 & 1.258147 & 0.003759 \\
\hline 15.238129 & -1.258147 & -0.003759 \\
\hline-15.238129 & 1.258147 & -0.003759 \\
\hline-15.238129 & -1.258147 & 0.003759 \\
\hline
\end{tabular}

Table S14. The optimized Cartesian coordinates of the TTC ribbon linked through four-membered ring with $\mathrm{n}=4$ (type III) in the ground singlet state calculated at the B3LYP/6-31(d) level of theory

\begin{tabular}{|c|c|c|c|c|}
\hline \multirow{2}{*}{$\begin{array}{l}\text { Center } \\
\text { Number }\end{array}$} & \multirow{2}{*}{$\begin{array}{l}\text { Atomic } \\
\text { Number }\end{array}$} & \multicolumn{3}{|c|}{ Coordinates (Angstroms) } \\
\hline & & $\mathrm{X}$ & $\mathrm{Y}$ & Z \\
\hline 1 & 6 & -14.350667 & 4.013615 & 1.546632 \\
\hline 2 & 6 & -4.335569 & 4.204265 & 0.841074 \\
\hline 3 & 6 & 5.664095 & 4.128338 & -1.157831 \\
\hline 4 & 6 & 15.707285 & 4.067179 & -1.390286 \\
\hline 5 & 6 & -15.707285 & -4.067179 & -1.390286 \\
\hline 6 & 6 & -5.664095 & -4.128338 & -1.157831 \\
\hline 7 & 6 & 4.335569 & -4.204265 & 0.841074 \\
\hline 8 & 6 & 14.350667 & -4.013615 & 1.546632 \\
\hline 9 & 6 & -19.316489 & 0.665204 & 0.155727 \\
\hline 10 & 6 & -9.248158 & 0.622604 & 0.311842 \\
\hline 11 & 6 & 0.753611 & 0.696350 & -0.000726 \\
\hline 12 & 6 & 10.755838 & 0.621652 & -0.311878 \\
\hline 13 & 6 & -10.755838 & -0.621652 & -0.311878 \\
\hline 14 & 6 & -0.753611 & -0.696350 & -0.000726 \\
\hline 15 & 6 & 9.248158 & -0.622604 & 0.311842 \\
\hline 16 & 6 & 19.316489 & -0.665204 & 0.155727 \\
\hline 17 & 6 & -14.350667 & -4.013615 & -1.546632 \\
\hline
\end{tabular}




\begin{tabular}{|c|c|c|c|c|}
\hline 18 & 6 & -4.335569 & -4.204265 & -0.841074 \\
\hline 19 & 6 & 5.664095 & -4.128338 & 1.157831 \\
\hline 20 & 6 & 15.707285 & -4.067179 & 1.390286 \\
\hline 21 & 6 & -15.707285 & 4.067179 & 1.390286 \\
\hline 22 & 6 & -5.664095 & 4.128338 & 1.157831 \\
\hline 23 & 6 & 4.335569 & 4.204265 & -0.841074 \\
\hline 24 & 6 & 14.350667 & 4.013615 & -1.546632 \\
\hline 25 & 6 & -19.316489 & -0.665204 & -0.155727 \\
\hline 26 & 6 & -9.248158 & -0.622604 & -0.311842 \\
\hline 27 & 6 & 0.753611 & -0.696350 & 0.000726 \\
\hline 28 & 6 & 10.755838 & -0.621652 & 0.311878 \\
\hline 29 & 6 & -10.755838 & 0.621652 & 0.311878 \\
\hline 30 & 6 & -0.753611 & 0.696350 & 0.000726 \\
\hline 31 & 6 & 9.248158 & 0.622604 & -0.311842 \\
\hline 32 & 6 & 19.316489 & 0.665204 & -0.155727 \\
\hline 33 & 6 & -16.787109 & 0.695302 & 0.197439 \\
\hline 34 & 6 & -6.771536 & 0.668338 & 0.267706 \\
\hline 35 & 6 & 3.229836 & 0.717125 & -0.063094 \\
\hline 36 & 6 & 13.237385 & 0.658353 & -0.295896 \\
\hline 37 & 6 & -13.237385 & -0.658353 & -0.295896 \\
\hline 38 & 6 & -3.229836 & -0.717125 & -0.063094 \\
\hline 39 & 6 & 6.771536 & -0.668338 & 0.267706 \\
\hline 40 & 6 & 16.787109 & -0.695302 & 0.197439 \\
\hline 41 & 6 & -15.737818 & -1.679543 & -0.554964 \\
\hline 42 & 6 & -5.717062 & -1.684765 & -0.511871 \\
\hline 43 & 6 & 4.283969 & -1.733943 & 0.306535 \\
\hline 44 & 6 & 14.292700 & -1.644107 & 0.654492 \\
\hline 45 & 6 & -14.292700 & 1.644107 & 0.654492 \\
\hline 46 & 6 & -4.283969 & 1.733943 & 0.306535 \\
\hline 47 & 6 & 5.717062 & 1.684765 & -0.511871 \\
\hline 48 & 6 & 15.737818 & 1.679543 & -0.554964 \\
\hline 49 & 6 & -16.787109 & -0.695302 & -0.197439 \\
\hline 50 & 6 & -6.771536 & -0.668338 & -0.267706 \\
\hline 51 & 6 & 3.229836 & -0.717125 & 0.063094 \\
\hline 52 & 6 & 13.237385 & -0.658353 & 0.295896 \\
\hline 53 & 6 & -13.237385 & 0.658353 & 0.295896 \\
\hline 54 & 6 & -3.229836 & 0.717125 & 0.063094 \\
\hline 55 & 6 & 6.771536 & 0.668338 & -0.267706 \\
\hline 56 & 6 & 16.787109 & 0.695302 & -0.197439 \\
\hline 57 & 6 & -15.737818 & 1.679543 & 0.554964 \\
\hline 58 & 6 & -5.717062 & 1.684765 & 0.511871 \\
\hline 59 & 6 & 4.283969 & 1.733943 & -0.306535 \\
\hline 60 & 6 & 14.292700 & 1.644107 & -0.654492 \\
\hline 61 & 6 & -14.292700 & -1.644107 & -0.654492 \\
\hline 62 & 6 & -4.283969 & -1.733943 & -0.306535 \\
\hline 63 & 6 & 5.717062 & -1.684765 & 0.511871 \\
\hline 64 & 6 & 15.737818 & -1.679543 & 0.554964 \\
\hline 65 & 6 & -18.077971 & 1.291798 & 0.325639 \\
\hline 66 & 6 & -8.064304 & 1.253420 & 0.585047 \\
\hline 67 & 6 & 1.937137 & 1.382670 & -0.042364 \\
\hline 68 & 6 & 11.939514 & 1.242898 & -0.602400 \\
\hline 69 & 6 & -11.939514 & -1.242898 & -0.602400 \\
\hline 70 & 6 & -1.937137 & -1.382670 & -0.042364 \\
\hline 71 & 6 & 8.064304 & -1.253420 & 0.585047 \\
\hline 72 & 6 & 18.077971 & -1.291798 & 0.325639 \\
\hline 73 & 6 & -16.347837 & -2.920151 & -0.915884 \\
\hline 74 & 6 & -6.305012 & -2.900536 & -0.976560 \\
\hline 75 & 6 & 3.695497 & -3.028037 & 0.443858 \\
\hline 76 & 6 & 13.699010 & -2.833816 & 1.178273 \\
\hline 77 & 6 & -13.699010 & 2.833816 & 1.178273 \\
\hline 78 & 6 & -3.695497 & 3.028037 & 0.443858 \\
\hline 79 & 6 & 6.305012 & 2.900536 & -0.976560 \\
\hline 80 & 6 & 16.347837 & 2.920151 & -0.915884 \\
\hline 81 & 6 & -18.077971 & -1.291798 & -0.325639 \\
\hline 82 & 6 & -8.064304 & -1.253420 & -0.585047 \\
\hline 83 & 6 & 1.937137 & -1.382670 & 0.042364 \\
\hline 84 & 6 & 11.939514 & -1.242898 & 0.602400 \\
\hline 85 & 6 & -11.939514 & 1.242898 & 0.602400 \\
\hline 86 & 6 & -1.937137 & 1.382670 & 0.042364 \\
\hline 87 & 6 & 8.064304 & 1.253420 & -0.585047 \\
\hline 88 & 6 & 18.077971 & 1.291798 & -0.325639 \\
\hline 89 & 6 & -16.347837 & 2.920151 & 0.915884 \\
\hline 90 & 6 & -6.305012 & 2.900536 & 0.976560 \\
\hline 91 & 6 & 3.695497 & 3.028037 & -0.443858 \\
\hline 92 & 6 & 13.699010 & 2.833816 & -1.178273 \\
\hline 93 & 6 & -13.699010 & -2.833816 & -1.178273 \\
\hline 94 & 6 & -3.695497 & -3.028037 & -0.443858 \\
\hline 95 & 6 & 6.305012 & -2.900536 & 0.976560 \\
\hline 96 & 6 & 16.347837 & -2.920151 & 0.915884 \\
\hline
\end{tabular}




$\begin{array}{rrr}-11.968482 & 2.816493 & 1.320344 \\ -1.979039 & 3.108498 & 0.177725 \\ 8.022169 & 2.854071 & -1.244526 \\ 18.075609 & 2.958998 & -0.793985 \\ -18.075609 & -2.958998 & -0.793985 \\ -8.022169 & -2.854071 & -1.244526 \\ 1.979039 & -3.108498 & 0.177725 \\ 11.968482 & -2.816493 & 1.320344 \\ -18.075609 & 2.958998 & 0.793985 \\ -8.022169 & 2.854071 & 1.244526 \\ 1.979039 & 3.108498 & -0.177725 \\ 11.968482 & 2.816493 & -1.320344 \\ -11.968482 & -2.816493 & -1.320344 \\ -1.979039 & -3.108498 & -0.177725 \\ 8.022169 & -2.854071 & 1.244526 \\ 18.075609 & -2.958998 & 0.793985 \\ -13.782340 & 4.857379 & 1.925299 \\ -3.777533 & 5.131381 & 0.925825 \\ 6.221802 & 4.992964 & -1.503563 \\ 16.280638 & 4.954465 & -1.639851 \\ -16.280638 & 4.954465 & 1.639851 \\ -6.221802 & 4.992964 & 1.503563 \\ 3.777533 & 5.131381 & -0.925825 \\ 13.782340 & 4.857379 & -1.925299 \\ -13.782340 & -4.857379 & -1.925299 \\ -3.777533 & -5.131381 & -0.925825 \\ 6.221802 & -4.992964 & 1.503563 \\ 16.280638 & -4.954465 & 1.639851 \\ -16.280638 & -4.954465 & -1.639851 \\ -6.221802 & -4.992964 & -1.503563 \\ 3.777533 & -5.131381 & 0.925825 \\ 13.782340 & -4.857379 & 1.925299 \\ 20.237241 & 1.224707 & -0.288093 \\ 20.237241 & -1.224707 & 0.288093 \\ -20.237241 & 1.224707 & 0.288093 \\ -20.237241 & -1.224707 & -0.288093\end{array}$

Table S15. The optimized Cartesian coordinates of the TTC ribbon linked through four-membered ring with $n=5$ (type III) in the ground singlet state calculated at the B3LYP/6-31(d) level of theory

\begin{tabular}{|c|c|c|c|c|}
\hline \multirow{2}{*}{$\begin{array}{l}\text { Center } \\
\text { Number }\end{array}$} & \multirow{2}{*}{$\begin{array}{l}\text { Atomic } \\
\text { Number }\end{array}$} & \multicolumn{3}{|c|}{ Coordinates (Angstroms) } \\
\hline & & $\mathrm{X}$ & $Y$ & Z \\
\hline 1 & 6 & -19.357191 & 4.264744 & 0.561132 \\
\hline 2 & 6 & -9.340537 & 4.286013 & -0.151843 \\
\hline 3 & 6 & 0.665545 & 4.286075 & 0.156978 \\
\hline 4 & 6 & 10.671061 & 4.285427 & -0.164733 \\
\hline 5 & 6 & 20.714215 & 4.279768 & -0.400159 \\
\hline 6 & 6 & -20.714215 & -4.279768 & -0.400159 \\
\hline 7 & 6 & -10.671061 & -4.285427 & -0.164733 \\
\hline 8 & 6 & -0.665545 & -4.286075 & 0.156978 \\
\hline 9 & 6 & 9.340537 & -4.286013 & -0.151843 \\
\hline 10 & 6 & 19.357191 & -4.264744 & 0.561132 \\
\hline 11 & 6 & $-24 \cdot 323432$ & 0.683168 & -0.002699 \\
\hline 12 & 6 & -14.254611 & 0.678663 & 0.155410 \\
\hline 13 & 6 & -4.249215 & 0.679352 & -0.152026 \\
\hline 14 & 6 & 5.756816 & 0.679383 & 0.152050 \\
\hline 15 & 6 & 15.762326 & 0.677828 & -0.155720 \\
\hline 16 & 6 & -15.762326 & -0.677828 & -0.155720 \\
\hline 17 & 6 & -5.756816 & -0.679383 & 0.152050 \\
\hline 18 & 6 & 4.249215 & -0.679352 & -0.152026 \\
\hline 19 & 6 & 14.254611 & -0.678663 & 0.155410 \\
\hline 20 & 6 & 24.323432 & -0.683168 & -0.002699 \\
\hline 21 & 6 & -19.357191 & -4.264744 & -0.561132 \\
\hline 22 & 6 & -9.340537 & -4.286013 & 0.151843 \\
\hline 23 & 6 & 0.665545 & -4.286075 & -0.156978 \\
\hline 24 & 6 & 10.671061 & -4.285427 & 0.164733 \\
\hline 25 & 6 & 20.714215 & -4.279768 & 0.400159 \\
\hline 26 & 6 & -20.714215 & 4.279768 & 0.400159 \\
\hline 27 & 6 & -10.671061 & 4.285427 & 0.164733 \\
\hline 28 & 6 & -0.665545 & 4.286075 & -0.156978 \\
\hline
\end{tabular}




\begin{abstract}
9.340537
19.357191

$-24.323432$

$-14.254611$

$-4.249215$

5.756816

15.762326

$-15.762326$

$-5.756816$

4. 249215

14.254611

24.323432

$-21.793962$

$-11.777500$

$-1.772035$

8.233822
\end{abstract}

18.243945

$-18.243945$

$-8.233822$

1.772035

11.777500

21.793962

$-20.744638$

$-10.722881$

$-0.717330$

9. 288544

19.299315

$-19.299315$

$-9.288544$

0.717330

10.722881

20.744638

$-21.793962$

$-11.777500$

$-1.772035$

8.233822

18.243945

$-18.243945$

$-8.233822$

1.772035

11.777500

21.793962

$-20.744638$

$-10.722881$

$-0.717330$

9.288544

19.299315

$-19.299315$

$-9.288544$

0.717330

10.722881

20.744638

$-23.084899$

$-13.070671$

$-3.065419$

6.940537

16.945994

$-16.945994$

$-6.940537$

3. 065419

13.070671

23.084899

$-21.354814$

$-11.311807$

$-1.306429$

8.699701

18.705477

$-18.705477$

$-8.699701$

1.306429

11.311807

21.354814

$-23.084899$

$-13.070671$

$-3.065419$

6.940537

16.945994

$-16.945994$

$-6.940537$

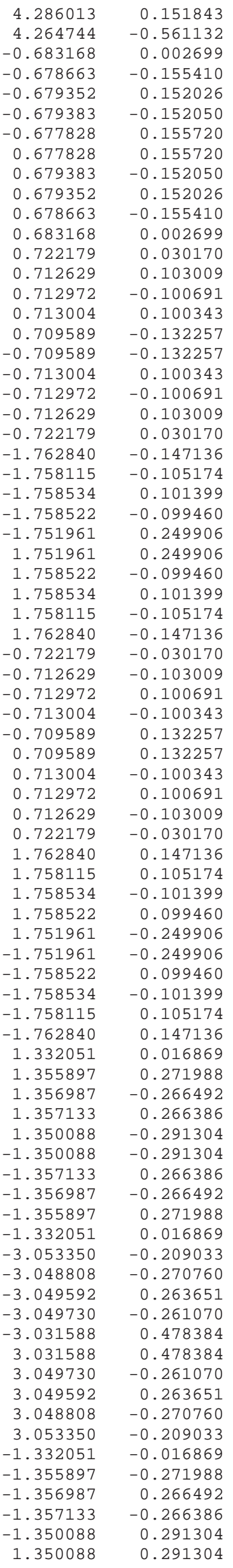

$-0.266386$ 
$-3.049592$

$-3.048808$

$-3.053350$

3. 048589

3.068625

3.068354

3.066630

3.062424

$-3.062424$

$-3.066630$

$-3.068354$

$-3.068625$

$-3.048589$

3.062424

3.066630

3.068354

3.068625

3.048589

$-3.048589$

$-3.068625$

$-3.068354$

$-3.066630$

$-3.062424$

5.173919

5.207484

5.207308

5.206461

5.200664

5.200664

5.206461

5.207308

5.207484

5.173919

$-5.173919$

$-5.207484$

$-5.207308$

$-5.206461$

$-5.200664$

$-5.200664$

$-5.206461$

$-5.207308$

$-5.207484$

$-5.173919$

1.258116

$-1.258116$

1.258116

0.266492

$-0.271988$

$-0.016869$

0.209033

0.270760

$-0.263651$

0.261070

$-0.478384$

$-0.478384$

0.261070

$-0.263651$

0.270760

0.209033

0.617704

$-0.524299$

0.524910

$-0.535657$

$-0.084192$

$-0.084192$

$-0.535657$

0.524910

$-0.524299$

0.617704

0.084192

0.535657

$-0.524910$

0.524299

$-0.617704$

$-0.617704$

0.524299

$-0.524910$

0.535657

0.084192

0.729976

$-0.280965$

0.286336

$-0.296454$

$-0.436220$

0.436220

0.296454

$-0.286336$

0.280965

$-0.729976$

$-0.729976$

0.280965

$-0.286336$

0.296454

0.436220

$-0.436220$

$-0.296454$

0.286336

$-0.280965$

0.729976

$-0.003651$

0.003651

0.003651

25.244183

$-1.258116$

$-0.003651$

Table S16. The optimized Cartesian coordinates of the tetraselena[8]circulene (TSC) in the ground singlet state calculated at the B3LYP/6-31(d) level of theory

\begin{tabular}{|c|c|c|c|c|}
\hline \multirow{2}{*}{$\begin{array}{l}\text { Center } \\
\text { Number }\end{array}$} & \multirow{2}{*}{$\begin{array}{l}\text { Atomic } \\
\text { Number }\end{array}$} & \multicolumn{3}{|c|}{ Coordinates (Angstroms) } \\
\hline & & $\mathrm{X}$ & $\mathrm{Y}$ & Z \\
\hline 1 & 6 & -0.108462 & 4.308998 & -0.279297 \\
\hline 2 & 6 & -4.159280 & 1.127542 & -0.291498 \\
\hline 3 & 6 & -0.484596 & 1.820847 & -0.186093 \\
\hline 4 & 6 & -1.653225 & 0.903211 & -0.188608 \\
\hline 5 & 6 & -0.874802 & 3.160106 & -0.480392 \\
\hline 6 & 6 & -2.860701 & 1.600087 & -0.486597 \\
\hline 7 & 34 & -2.647581 & 3.374998 & -1.019904 \\
\hline 8 & 1 & -0.512822 & 5.288481 & -0.514367 \\
\hline 9 & 1 & -5.014111 & 1.752443 & -0.530076 \\
\hline
\end{tabular}




$\begin{array}{rrrrr}10 & 6 & 4.308998 & 0.108462 & 0.279297 \\ 11 & 6 & 1.127542 & 4.159280 & 0.291498 \\ 12 & 6 & 1.820847 & 0.484596 & 0.186093 \\ 13 & 6 & 0.903211 & 1.653225 & 0.188608 \\ 14 & 6 & 3.160106 & 0.874802 & 0.480392 \\ 15 & 6 & 1.600087 & 2.860701 & 0.486597 \\ 16 & 34 & 3.374998 & 2.647581 & 1.019904 \\ 17 & 1 & 5.288481 & 0.512822 & 0.514367 \\ 18 & 1 & 1.752443 & 5.014111 & 0.530076 \\ 19 & 6 & -4.308998 & -0.108462 & 0.279297 \\ 20 & 6 & -1.127542 & -4.159280 & 0.291498 \\ 21 & 6 & -1.820847 & -0.484596 & 0.186093 \\ 22 & 6 & -0.903211 & -1.653225 & 0.188608 \\ 23 & 6 & -3.160106 & -0.874802 & 0.480392 \\ 24 & 6 & -1.600087 & -2.860701 & 0.486597 \\ 25 & 34 & -3.374998 & -2.647581 & 1.019904 \\ 26 & 1 & -5.288481 & -0.512822 & 0.514367 \\ 27 & 1 & -1.752443 & -5.014111 & 0.530076 \\ 28 & 6 & 0.108462 & -4.308998 & -0.279297 \\ 29 & 6 & 4.159280 & -1.127542 & -0.291498 \\ 30 & 6 & 0.484596 & -1.820847 & -0.186093 \\ 31 & 6 & 1.653225 & -0.903211 & -0.188608 \\ 32 & 6 & 0.874802 & -3.160106 & -0.480392 \\ 33 & 6 & 2.860701 & -1.600087 & -0.486597 \\ 34 & 34 & 2.647581 & -3.374998 & -1.019904 \\ 35 & 1 & 5.014111 & -1.752443 & -0.530076 \\ 36 & 1 & 0.512822 & -5.288481 & -0.514367 \\ --------------------------------------------------\end{array}$

Table S17. The optimized Cartesian coordinates of the directly fused TSC-based ribbon with $\mathrm{n}=2$ (type $\mathbf{I}$ ) in the ground singlet state calculated at the B3LYP/6-31(d) level of theory

\begin{tabular}{|c|c|c|c|c|}
\hline \multirow{2}{*}{$\begin{array}{l}\text { Center } \\
\text { Number }\end{array}$} & \multirow{2}{*}{$\begin{array}{l}\text { Atomic } \\
\text { Number }\end{array}$} & \multicolumn{3}{|c|}{ Coordinates (Angstroms) } \\
\hline & & $\mathrm{X}$ & $\mathrm{Y}$ & Z \\
\hline 1 & 6 & 4.931747 & -1.216252 & -1.261462 \\
\hline 2 & 6 & -5.982423 & -0.410233 & 0.595217 \\
\hline 3 & 6 & -4.931542 & -1.216097 & 1.261893 \\
\hline 4 & 6 & 5.982541 & -0.410429 & -0.594600 \\
\hline 5 & 6 & -4.931721 & 1.215970 & -1.261833 \\
\hline 6 & 6 & 5.982398 & 0.410278 & 0.595334 \\
\hline 7 & 6 & 4.931515 & 1.216378 & 1.261720 \\
\hline 8 & 6 & -5.982517 & 0.410209 & -0.594899 \\
\hline 9 & 6 & 3.546508 & 1.477396 & 0.940349 \\
\hline 10 & 6 & -2.508202 & 0.685719 & -0.251854 \\
\hline 11 & 6 & -3.546635 & 1.477034 & -0.940838 \\
\hline 12 & 6 & 2.508160 & 0.685842 & 0.251513 \\
\hline 13 & 6 & -3.546505 & -1.477140 & 0.940669 \\
\hline 14 & 6 & 2.508200 & -0.685731 & -0.251685 \\
\hline 15 & 6 & 3.546633 & -1.477199 & -0.940496 \\
\hline 16 & 6 & -2.508159 & -0.685742 & 0.251651 \\
\hline 17 & 6 & 5.466159 & 2.006840 & 2.318804 \\
\hline 18 & 6 & -1.251838 & -1.326509 & 0.242727 \\
\hline 19 & 6 & -5.466522 & 2.006025 & -2.319140 \\
\hline 20 & 6 & 1.251888 & -1.326510 & -0.242819 \\
\hline 21 & 6 & -5.466214 & -2.006304 & 2.319151 \\
\hline 22 & 6 & 1.251845 & 1.326624 & 0.242414 \\
\hline 23 & 6 & 5.466577 & -2.006525 & -2.318592 \\
\hline 24 & 6 & -1.251896 & 1.326515 & -0.243097 \\
\hline 25 & 6 & 3.001998 & -2.667511 & -1.499319 \\
\hline 26 & 6 & -7.262255 & 0.635038 & -1.180071 \\
\hline 27 & 6 & -3.001832 & -2.667469 & 1.499427 \\
\hline 28 & 6 & 7.262009 & 0.635170 & 1.180757 \\
\hline 29 & 6 & -3.002020 & 2.667269 & -1.499845 \\
\hline 30 & 6 & 7.262314 & -0.635469 & -1.179613 \\
\hline 31 & 6 & 3.001856 & 2.667898 & 1.498757 \\
\hline 32 & 6 & -7.262070 & -0.635078 & 1.180580 \\
\hline 33 & 6 & -4.836752 & 3.087878 & -2.937939 \\
\hline 34 & 6 & 4.836367 & 3.089038 & 2.936974 \\
\hline 35 & 6 & 0.000011 & -0.696377 & -0.000113 \\
\hline 36 & 6 & 4.836784 & -3.088444 & -2.937251 \\
\hline 37 & 6 & -4.836398 & -3.088292 & 2.937668 \\
\hline
\end{tabular}




$\begin{array}{rrr}-0.000010 & 0.696442 & -0.000268 \\ 3.612042 & -3.477428 & -2.458072 \\ -3.611772 & -3.477364 & 2.458264 \\ -8.493165 & 0.288104 & -0.621971 \\ 8.493029 & 0.287935 & 0.623086 \\ -3.612056 & 3.477003 & -2.458757 \\ 3.611788 & 3.478039 & 2.457392 \\ -8.493065 & -0.288074 & 0.622711 \\ 8.493200 & -0.288519 & -0.621469 \\ 7.248931 & -1.649993 & -2.748208 \\ -7.248404 & -1.649517 & 2.749226 \\ -7.248812 & 1.649284 & -2.748846 \\ 7.248282 & 1.650026 & 2.749133 \\ 1.249073 & 3.044445 & 0.989749 \\ -1.249181 & 3.044013 & -0.991184 \\ -1.249029 & -3.044115 & 0.990561 \\ 1.249140 & -3.044130 & -0.990630 \\ 3.102759 & -4.353397 & -2.847619 \\ 3.102521 & 4.354183 & 2.846569 \\ -3.102792 & 4.352929 & -2.848428 \\ -5.340674 & 3.641906 & -3.723549 \\ -5.340235 & -3.642449 & 3.723241 \\ -3.102483 & -4.353375 & 2.847711 \\ 5.340723 & -3.642633 & -3.722737 \\ 5.340185 & 3.643386 & 3.722425 \\ 9.418372 & -0.527897 & -1.136159 \\ 9.418062 & 0.527165 & 1.138095 \\ -9.418128 & -0.527259 & 1.137689 \\ -9.418308 & 0.527301 & -1.136798\end{array}$

Table S18. The optimized Cartesian coordinates of the directly fused TSC-based ribbon with $\mathrm{n}=3$ (type $\mathbf{I}$ ) in the ground singlet state calculated at the B3LYP/6-31(d) level of theory

\begin{tabular}{|c|c|c|c|c|}
\hline \multirow{2}{*}{$\begin{array}{l}\text { Center } \\
\text { Number }\end{array}$} & \multirow{2}{*}{$\begin{array}{l}\text { Atomic } \\
\text { Number }\end{array}$} & \multicolumn{3}{|c|}{ Coordinates (Angstroms) } \\
\hline & & $\mathrm{x}$ & Y & Z \\
\hline 1 & 6 & -7.396946 & 1.713764 & -0.997754 \\
\hline 2 & 6 & 8.840250 & 1.722917 & -0.994605 \\
\hline 3 & 6 & -1.715986 & 0.725691 & 1.327414 \\
\hline 4 & 6 & -0.720205 & 1.687129 & 1.851987 \\
\hline 5 & 6 & -6.396488 & 0.727432 & -0.516523 \\
\hline 6 & 6 & 9.858106 & 0.721085 & -0.589752 \\
\hline 7 & 6 & -0.720205 & -1.687129 & 1.851987 \\
\hline 8 & 6 & -6.396488 & -0.727432 & -0.516523 \\
\hline 9 & 6 & 9.858106 & -0.721085 & -0.589752 \\
\hline 10 & 6 & -7.396946 & -1.713764 & -0.997754 \\
\hline 11 & 6 & 8.840250 & -1.722917 & -0.994605 \\
\hline 12 & 6 & -1.715986 & -0.725691 & 1.327414 \\
\hline 13 & 6 & -8.840250 & -1.722917 & -0.994605 \\
\hline 14 & 6 & 7.396946 & -1.713764 & -0.997754 \\
\hline 15 & 6 & 1.715986 & -0.725691 & 1.327414 \\
\hline 16 & 6 & 0.720205 & -1.687129 & 1.851987 \\
\hline 17 & 6 & -9.858106 & -0.721085 & -0.589752 \\
\hline 18 & 6 & 6.396488 & -0.727432 & -0.516523 \\
\hline 19 & 6 & 0.720205 & 1.687129 & 1.851987 \\
\hline 20 & 6 & -9.858106 & 0.721085 & -0.589752 \\
\hline 21 & 6 & 6.396488 & 0.727432 & -0.516523 \\
\hline 22 & 6 & -8.840250 & 1.722917 & -0.994605 \\
\hline 23 & 6 & 7.396946 & 1.713764 & -0.997754 \\
\hline 24 & 6 & 1.715986 & 0.725691 & 1.327414 \\
\hline 25 & 6 & -6.771700 & -2.940378 & -1.355354 \\
\hline 26 & 6 & 9.442642 & -2.977729 & -1.299632 \\
\hline 27 & 6 & 2.942969 & 1.346992 & 1.012077 \\
\hline 28 & 6 & -1.340103 & -2.900927 & 2.252303 \\
\hline 29 & 6 & -11.113484 & 1.336573 & -0.315145 \\
\hline 30 & 6 & 5.162155 & 1.340901 & -0.204900 \\
\hline 31 & 6 & -1.340103 & 2.900927 & 2.252303 \\
\hline 32 & 6 & -11.113484 & -1.336573 & -0.315145 \\
\hline 33 & 6 & 5.162155 & -1.340901 & -0.204900 \\
\hline 34 & 6 & -6.771700 & 2.940378 & -1.355354 \\
\hline 35 & 6 & 9.442642 & 2.977729 & -1.299632 \\
\hline
\end{tabular}




\begin{tabular}{|c|c|c|}
\hline 2.942969 & -1.346992 & 1.012077 \\
\hline-9.442642 & 2.977729 & -1.299632 \\
\hline 6.771700 & 2.940378 & -1.355354 \\
\hline-2.942969 & -1.346992 & 1.012077 \\
\hline 1.340103 & 2.900927 & 2.25230 \\
\hline-5.162155 & -1.340901 & -0.20490 \\
\hline 11.113484 & -1.336573 & -0.31514 \\
\hline 1.340103 & -2.900927 & 2.25230 \\
\hline-5.162155 & 1.340901 & -0.20 \\
\hline 11.113484 & 1.336573 & -0.3151 \\
\hline-9.442642 & -2.977729 & -1.2996 \\
\hline 6.771700 & -2.940378 & -1.3553 \\
\hline-2.942969 & 1.346992 & 1.012 \\
\hline-0.6863 & -3.993395 & 2.826 \\
\hline-7.416197 & -4.074459 & -1.8521 \\
\hline 8.786423 & -4.097652 & -1.8127 \\
\hline 12.260 & 0.685798 & 0.14 \\
\hline 4.050245 & 0.699456 & 0.4017 \\
\hline-7.416197 & 4.074459 & -1.85219 \\
\hline 8.786423 & 4.097652 & -1.81274 \\
\hline-0.686387 & 3.993395 & $2.8267 \varepsilon$ \\
\hline 12.260547 & -0.685798 & 0.14027 \\
\hline 4.050245 & -0.699456 & 0.40174 \\
\hline-8.786423 & 4.097652 & -1.8127 \\
\hline 7.416197 & 4.074459 & -1.85219 \\
\hline 0.686387 & 3.993395 & 2.82678 \\
\hline-4.050245 & -0.699456 & 0.4017 \\
\hline 12.260547 & -0.685798 & 0.14027 \\
\hline 0.686387 & -3.993395 & 2.82678 \\
\hline-8.786423 & -4.097652 & -1.81274 \\
\hline 7.416197 & -4.074459 & -1.85219 \\
\hline-4.050245 & 0.699456 & 0.40174 \\
\hline 12.260547 & 0.685798 & 0.14027 \\
\hline-4.972172 & 3.075601 & -0.88460 \\
\hline 11.235072 & 3.134254 & -0.80359 \\
\hline-3.125838 & 3.072039 & 1.73065 \\
\hline-3.125838 & -3.072039 & 1.7306 \\
\hline-4.972172 & -3.075601 & -0.88460 \\
\hline 11.235072 & -3.134254 & -0.80359 \\
\hline 11.235072 & -3.134254 & -0.80359 \\
\hline 4.972172 & -3.075601 & -0.88460 \\
\hline 3.125838 & -3.072039 & 1.73065 \\
\hline 3.125838 & 3.072039 & 1.73065 \\
\hline 11.235072 & 3.134254 & -0.80359 \\
\hline 4.972172 & 3.075601 & -0.88460 \\
\hline-9.346596 & 4.984991 & -2.09139 \\
\hline 6.844225 & 4.943811 & -2.16238 \\
\hline-9.346596 & -4.984991 & -2.09139 \\
\hline 6.844225 & -4.943811 & -2.16238 \\
\hline 1.249397 & -4.855900 & 3.17078 \\
\hline-1.249397 & -4.855900 & 3.17078 \\
\hline-1.249397 & 4.855900 & 3.17078 \\
\hline 1.249397 & 4.855900 & 3.17078 \\
\hline-6.844225 & 4.943811 & -2.16238 \\
\hline 9.346596 & 4.984991 & -2.09139 \\
\hline-6.844225 & -4.943811 & -2.16238 \\
\hline 9.346596 & -4.984991 & -2.09139 \\
\hline 13.151176 & 1.251879 & 0.39560 \\
\hline 13.151176 & -1.251879 & 0.39560 \\
\hline 13.151176 & 1.251879 & 0.39560 \\
\hline 13.151176 & -1.251879 & 0.39560 \\
\hline
\end{tabular}

Table S19. The optimized Cartesian coordinates of the directly fused TSC-based ribbon with $\mathrm{n}=4$ (type $\mathbf{I}$ ) in the ground singlet state calculated at the B3LYP/6-31(d) level of theory

\begin{tabular}{|c|c|c|c|c|}
\hline \multirow{2}{*}{$\begin{array}{l}\text { Center } \\
\text { Number }\end{array}$} & \multirow{2}{*}{$\begin{array}{l}\text { Atomic } \\
\text { Number }\end{array}$} & \multicolumn{3}{|c|}{ Coordinates (Angstroms) } \\
\hline & & $\mathrm{X}$ & $\mathrm{Y}$ & Z \\
\hline 1 & 6 & -1.620418 & -3.249466 & 1.690180 \\
\hline 2 & 6 & -0.672641 & 12.957376 & 1.722721 \\
\hline 3 & 6 & 0.208725 & -13.949787 & 0.721068 \\
\hline
\end{tabular}




\begin{tabular}{|c|c|c|}
\hline 1.048176 & 2.283219 & 0.725790 \\
\hline 0.672650 & -12.957373 & 1.722721 \\
\hline 1.620408 & 3.249464 & 1.690180 \\
\hline-1.048187 & -2.283221 & \\
\hline-0.208714 & 13.949789 & 0.721068 \\
\hline 0.672650 & -12.957373 & -1.722721 \\
\hline 1.620408 & 3.249464 & -1.690180 \\
\hline-1.048187 & -2.283221 & \\
\hline-0.208714 & 13.94 & -0 . \\
\hline-1.620418 & -3.249466 & -1 \\
\hline-0.672641 & 12.957376 & -1.722721 \\
\hline 0.208725 & -13.949787 & -0.721068 \\
\hline 1.048176 & 2.28 & -0.7 \\
\hline-1.7 & -4.687791 & -1 . \\
\hline-0.760036 & 11.516745 & 713447 \\
\hline 0.336963 & -10.490091 & -0.727421 \\
\hline 1.237144 & 5.71 & -0.725844 \\
\hline 0042 & -11.51 & -1.713447 \\
\hline 1.701798 & 4.687 & -1.689238 \\
\hline-1.237151 & -5.711610 & -0.725844 \\
\hline-0.336959 & 10.490093 & -0.727421 \\
\hline 0042 & -11.51 & \\
\hline 1.701798 & 4.687789 & 1.689238 \\
\hline-1.237151 & -5.711610 & 0.725844 \\
\hline-0.336959 & 10.490093 & 0.727421 \\
\hline-1. & -4 . & 39238 \\
\hline-0.760036 & 11.51 & 713447 \\
\hline 0.336963 & -10.490 & 0.727421 \\
\hline 1.237144 & & 0.725844 \\
\hline-1.9 & -2 . & -2 \\
\hline 42298 & $13.5^{7}$ & -2 . \\
\hline 0.097294 & -9.23 & 1.340991 \\
\hline 0.991407 & 6.95 & 346645 \\
\hline 0.9 & -13.5 & -2 . \\
\hline 1.978762 & 2.609500 & 907140 \\
\hline-0.991410 & -6.954905 & 346645 \\
\hline-0.097293 & 9.239882 & 1.340991 \\
\hline 0.942307 & -13.57 & 7474 \\
\hline 1.978762 & 2.60 & 907140 \\
\hline-0.991410 & -6.954905 & 346645 \\
\hline-0.097293 & 9.239882 & 340991 \\
\hline 78771 & -2.60 & 90714 \\
\hline-0. & 13.5 & 177474 \\
\hline 0.097294 & -9.23 & 340991 \\
\hline 0.991407 & 6.954904 & -1.346645 \\
\hline-2.133019 & -5.28 & 04156 \\
\hline-1.1 & 10 . & 9694 \\
\hline-0.138860 & -15.18 & -1 . \\
\hline 0.667225 & 1.074338 & -1.345834 \\
\hline 1.154258 & -10.91 & 39694 \\
\hline 2.13 & 5.2 & 04156 \\
\hline-0.667235 & -1.07 & -1.345834 \\
\hline 0.138872 & 15.186893 & -1.336613 \\
\hline 1.154258 & -10.913304 & 39694 \\
\hline 2.1 & 5.28 & 904156 \\
\hline-0.667235 & -1.074340 & 1.345834 \\
\hline 0.138872 & 15.186893 & 1.336613 \\
\hline-2.133019 & -5.284618 & 904156 \\
\hline-1.154254 & 10.913308 & 39694 \\
\hline-0.138860 & -15.186891 & 1.336613 \\
\hline 0.667225 & 1.074338 & 1.345834 \\
\hline 1.493430 & -12.951232 & 97077 \\
\hline 34260 & 3.230235 & -4 \\
\hline-2.584268 & -3.230238 & 01903 \\
\hline-1.493422 & 12.951236 & -4.097076 \\
\hline-0.444909 & -8.095131 & \\
\hline 44908 & 8.095131 & 699396 \\
\hline-2.584268 & -3.230238 & 4.001903 \\
\hline-1.493422 & 12.951236 & 4.097076 \\
\hline 1.493430 & -12.951232 & 097077 \\
\hline 2.584260 & 3.230235 & 4.001903 \\
\hline-0.444909 & -8.095131 & -0.699396 \\
\hline 0.444908 & 8.095131 & -0.699396 \\
\hline-2.664051 & -4.600581 & 3.999975 \\
\hline-1.613104 & 11.585661 & 4.073636 \\
\hline 1.613109 & -11.585657 & 4.073636 \\
\hline 2.664044 & 4.600577 & 3.999975 \\
\hline-0.660771 & -16.305268 & -0.685809 \\
\hline & & \\
\hline
\end{tabular}




\begin{tabular}{|c|c|c|}
\hline 0.660784 & 16.305269 & -0.685809 \\
\hline 1.613109 & -11.585657 & -4.073636 \\
\hline 2.664044 & 4.600577 & -3.999975 \\
\hline-2.664051 & -4.600581 & -3.999975 \\
\hline 1.613104 & 11.585661 & -4.073636 \\
\hline 0.660771 & -16.305268 & \\
\hline 0.000005 & -0.000001 & 0.700388 \\
\hline 0.660784 & 16.305269 & 0.6858 \\
\hline-1.363205 & -0.8536 & 3.0 \\
\hline-0.342039 & 15.3367 & \\
\hline 0.342051 & -15.336778 & \\
\hline 1.363196 & 0.8536 & 3.0 \\
\hline 0.342 & -15.336 & -3 \\
\hline 1.36 & 0.8 & -3 \\
\hline-1.363205 & -0.853690 & -3.0 \\
\hline-0.342039 & 15.3367 & -3 \\
\hline-1.7 & -7.0 & -3 \\
\hline-0.78 & 9.08 & -3 \\
\hline & -9 & -3 \\
\hline 1.71 & 7.0 & -3 . \\
\hline 0.78 & -9.0894 & \\
\hline 1.716024 & 7.09 & 3. \\
\hline-1.71 & -7 & \\
\hline-0.788694 & 9.0894 & \\
\hline-3.036344 & -5.144209 & 4.863 \\
\hline-1.9 & 11.0 & \\
\hline-3.036344 & -5.14 & -4 \\
\hline-1.956610 & 11.032748 & -4 . \\
\hline 1.9 & -11.032 & -4 . \\
\hline 3.036338 & 5.144205 & -4.8 \\
\hline 1.739128 & -13.526612 & -4.98 \\
\hline 2.8 & 2.6 & -4. \\
\hline 1.739128 & -13.526612 & 4.9844 \\
\hline 2.891360 & 2.649351 & 4.86644 \\
\hline 56614 & -11.032743 & 4.94 \\
\hline 3.036338 & 5.144205 & 4.8631 \\
\hline-2.891368 & -2.649355 & 4.86645 \\
\hline-1.739120 & 13.526617 & 4.98441 \\
\hline-2.891368 & -2.649355 & -4.86645 \\
\hline-1.739120 & 13.526617 & -4.98441 \\
\hline & 17.179420 & 1.25186 \\
\hline 0.967864 & 17.179420 & -1.25186 \\
\hline-0.967850 & -17.179419 & 1.25186 \\
\hline-0.967850 & -17.179419 & -1.25186 \\
\hline
\end{tabular}

Table S20. The optimized Cartesian coordinates of the directly fused TSC-based ribbon with $\mathrm{n}=5$ (type $\mathbf{I}$ ) in the ground singlet state calculated at the B3LYP/6-31(d) level of theory

\begin{tabular}{|c|c|c|c|c|}
\hline \multirow{2}{*}{$\begin{array}{l}\text { Center } \\
\text { Number }\end{array}$} & \multirow{2}{*}{$\begin{array}{l}\text { Atomic } \\
\text { Number }\end{array}$} & \multicolumn{3}{|c|}{ Coordinates (Angstroms) } \\
\hline & & $\mathrm{X}$ & $\mathrm{Y}$ & Z \\
\hline 1 & 6 & -15.508882 & 1.713637 & -1.163101 \\
\hline 2 & 6 & 0.720396 & 1.692146 & -1.181136 \\
\hline 3 & 6 & 16.952197 & 1.722873 & -1.159909 \\
\hline 4 & 6 & -9.828145 & 0.725832 & 1.164099 \\
\hline 5 & 6 & 6.394911 & 0.725776 & 1.169561 \\
\hline 6 & 6 & -8.832462 & 1.688906 & 1.686731 \\
\hline 7 & 6 & 7.391881 & 1.689780 & 1.687060 \\
\hline 8 & 6 & -14.508416 & 0.727433 & -0.681636 \\
\hline 9 & 6 & 1.717460 & 0.725941 & -0.666862 \\
\hline 10 & 6 & 17.970135 & 0.721086 & -0.755236 \\
\hline 11 & 6 & -8.832462 & -1.688906 & 1.686731 \\
\hline 12 & 6 & 7.391881 & -1.689780 & 1.687060 \\
\hline 13 & 6 & -14.508416 & -0.727433 & -0.681636 \\
\hline 14 & 6 & 1.717460 & -0.725941 & -0.666862 \\
\hline 15 & 6 & 17.970135 & -0.721086 & -0.755236 \\
\hline 16 & 6 & -15.508882 & -1.713637 & -1.163101 \\
\hline 17 & 6 & 0.720396 & -1.692146 & -1.181136 \\
\hline 18 & 6 & 16.952197 & -1.722873 & -1.159909 \\
\hline 19 & 6 & -9.828145 & -0.725832 & 1.164099 \\
\hline 20 & 6 & 6.394911 & -0.725776 & 1.169561 \\
\hline
\end{tabular}




\begin{tabular}{|c|c|c|}
\hline-7.427215 & 4.000715 & 2.652067 \\
\hline 8.799897 & 3.998941 & 2.653773 \\
\hline-12.162458 & -0.699404 & 0.237215 \\
\hline 4.056523 & -0.700344 & 0.251464 \\
\hline 20.372782 & -0.685795 & -0.025826 \\
\hline-7.427215 & -4.000715 & 2.652067 \\
\hline 8.799897 & -3.998941 & 2.653773 \\
\hline-16.898226 & -4.097639 & -1.978044 \\
\hline-0.686289 & -4.008010 & -2.135851 \\
\hline 15.528018 & -4.074287 & -2.017769 \\
\hline-12.162458 & 0.699404 & 0.237215 \\
\hline 4.056523 & 0.700344 & 0.251464 \\
\hline 20.372782 & 0.685795 & -0.025826 \\
\hline-13.084025 & 3.075344 & -1.050447 \\
\hline 3.127937 & 3.074869 & -1.058876 \\
\hline 19.346946 & 3.134336 & -0.969031 \\
\hline-11.239024 & 3.072503 & 1.564161 \\
\hline 4.985487 & 3.074033 & 1.566219 \\
\hline-11.239024 & -3.072503 & 1.564161 \\
\hline 4.985487 & -3.074033 & 1.566219 \\
\hline-13.084025 & -3.075344 & -1.050447 \\
\hline 3.127937 & -3.074869 & -1.058876 \\
\hline 19.346946 & -3.134336 & -0.969031 \\
\hline-19.346946 & -3.134336 & -0.969031 \\
\hline-3.127937 & -3.074869 & -1.058876 \\
\hline 13.084025 & -3.075344 & -1.050447 \\
\hline-4.985487 & -3.074033 & 1.566219 \\
\hline 11.239024 & -3.072503 & 1.564161 \\
\hline-4.985487 & 3.074033 & 1.566219 \\
\hline 11.239024 & 3.072503 & 1.564161 \\
\hline-19.346946 & 3.134336 & -0.969031 \\
\hline-3.127937 & 3.074869 & -1.058876 \\
\hline 13.084025 & 3.075344 & -1.050447 \\
\hline-17.458381 & 4.985020 & -2.256576 \\
\hline-1.249634 & 4.873139 & -2.472524 \\
\hline 14.955968 & 4.943557 & -2.328032 \\
\hline-17.458381 & -4.985020 & -2.256576 \\
\hline-1.249634 & -4.873139 & -2.472524 \\
\hline 14.955968 & -4.943557 & -2.328032 \\
\hline-6.864691 & -4.864991 & 2.992319 \\
\hline 9.363674 & -4.861966 & 2.995167 \\
\hline-9.363674 & -4.861966 & 2.995167 \\
\hline 6.864691 & -4.864991 & 2.992319 \\
\hline-9.363674 & 4.861966 & 2.995167 \\
\hline 6.864691 & 4.864991 & 2.992319 \\
\hline-6.864691 & 4.864991 & 2.992319 \\
\hline 9.363674 & 4.861966 & 2.995167 \\
\hline-14.955968 & 4.943557 & -2.328032 \\
\hline 1.249634 & 4.873139 & -2.472524 \\
\hline 17.458381 & 4.985020 & -2.256576 \\
\hline-14.955968 & -4.943557 & -2.328032 \\
\hline 1.249634 & -4.873139 & -2.472524 \\
\hline 17.458381 & -4.985020 & -2.256576 \\
\hline-21.263492 & 1.251893 & 0.229177 \\
\hline-21.263492 & -1.251893 & 0.229177 \\
\hline 21.263492 & 1.251893 & 0.229177 \\
\hline 21.263492 & -1.251893 & 0.229177 \\
\hline
\end{tabular}

Table S21. The optimized Cartesian coordinates of the TSC-based ribbon fused via benzene-core linker with $\mathrm{n}=2$ (type II) in the ground singlet state calculated at the B3LYP/6-31(d) level of theory

\begin{tabular}{|c|c|c|c|c|}
\hline \multirow{2}{*}{$\begin{array}{l}\text { Center } \\
\text { Number }\end{array}$} & \multirow{2}{*}{$\begin{array}{l}\text { Atomic } \\
\text { Number }\end{array}$} & \multicolumn{3}{|c|}{ Coordinates (Angstroms) } \\
\hline & & $\mathrm{X}$ & $\mathrm{Y}$ & $\mathrm{Z}$ \\
\hline 1 & 6 & 4.934469 & 4.274167 & 0.298617 \\
\hline 2 & 6 & -6.077018 & 4.268041 & -0.134283 \\
\hline 3 & 6 & 6.076828 & -4.268065 & 0.135918 \\
\hline 4 & 6 & -4.934031 & -4.274130 & -0.298337 \\
\hline 5 & 6 & 9.738537 & 0.572666 & -0.387241 \\
\hline 6 & 6 & -1.205628 & 0.610750 & -0.418503 \\
\hline 7 & 6 & 1.205657 & -0.610456 & 0.419235 \\
\hline
\end{tabular}




\begin{tabular}{|c|c|c|}
\hline-9.738811 & -0.573076 & 0.384427 \\
\hline 4.903446 & -4.195480 & 0.842283 \\
\hline-6.234210 & -4.196508 & -0.723889 \\
\hline 6.234898 & 4.196429 & 0.723378 \\
\hline-4.903447 & 4.195833 & -0.840371 \\
\hline 9.611946 & -0.674912 & -0.942352 \\
\hline-1.228326 & -0.658548 & 0.220022 \\
\hline 1.228354 & 0.658827 & -0.219321 \\
\hline-9.612630 & 0.674296 & 0.940095 \\
\hline 7.264506 & 0.661515 & 0.050194 \\
\hline-3.735269 & 0.673016 & -0.399544 \\
\hline 3.735284 & -0.672816 & 0.400095 \\
\hline-7.264479 & -0.661687 & -0.051353 \\
\hline 6.140099 & -1.762894 & -0.070301 \\
\hline-4.821020 & -1.768174 & -0.090416 \\
\hline 4.821183 & 1.768241 & 0.090520 \\
\hline-6.140299 & 1.762760 & 0.070642 \\
\hline 7.175006 & -0.731858 & -0.321621 \\
\hline-3.751647 & -0.744101 & -0.005616 \\
\hline 3.751704 & 0.744252 & 0.005995 \\
\hline-7.175293 & 0.731570 & 0.320963 \\
\hline 6.250473 & 1.711390 & 0.315611 \\
\hline-4.790298 & 1.711951 & -0.446549 \\
\hline 4.790268 & -1.711787 & 0.447295 \\
\hline-6.250200 & -1.711453 & -0.316245 \\
\hline 8.587694 & 1.189680 & 0.102637 \\
\hline-2.470441 & 1.194799 & -0.729504 \\
\hline 2.470468 & -1.194462 & 0.730315 \\
\hline-8.587603 & -1.189913 & -0.104814 \\
\hline 6.646386 & -3.074274 & -0.307423 \\
\hline-4.280927 & -3.082991 & 0.018185 \\
\hline 4.281133 & 3.083107 & -0.017720 \\
\hline-6.646720 & 3.074007 & 0.308217 \\
\hline 8.371193 & -1.304855 & -0.844245 \\
\hline-2.498841 & -1.307438 & 0.293157 \\
\hline 2.498880 & 1.307686 & -0.292507 \\
\hline-8.371818 & 1.304298 & 0.843106 \\
\hline 6.862375 & 2.951090 & 0.663623 \\
\hline-4.276687 & 2.952660 & -0.923886 \\
\hline 4.276709 & -2.952263 & 0.925295 \\
\hline-6.861791 & -2.951196 & -0.664648 \\
\hline-0.026004 & -1.239676 & 0.632819 \\
\hline 0.026037 & 1.239957 & -0.632112 \\
\hline 2.497557 & 3.155378 & -0.539554 \\
\hline-8.289512 & 3.137662 & 1.192834 \\
\hline 8.288690 & -3.138417 & -1.192902 \\
\hline-2.497509 & -3.155082 & 0.540588 \\
\hline 8.723686 & 2.910207 & 0.811191 \\
\hline-2.499265 & 2.901497 & -1.482937 \\
\hline 2.499328 & -2.900838 & 1.484474 \\
\hline-8.722993 & -2.910385 & -0.813628 \\
\hline-0.046680 & -2.220235 & 1.101034 \\
\hline 0.046740 & 2.220474 & -1.100416 \\
\hline 4.413534 & 5.224100 & 0.230632 \\
\hline-6.571504 & 5.216183 & 0.051504 \\
\hline 6.794271 & 5.082244 & 1.007279 \\
\hline-4.424159 & 5.084523 & -1.238822 \\
\hline 6.571236 & -5.216310 & -0.049540 \\
\hline-4.413074 & -5.224024 & -0.229974 \\
\hline 4.424241 & -5.083966 & 1.241290 \\
\hline-6.793355 & -5.082377 & -1.008070 \\
\hline 10.465891 & 1.198433 & 1.358998 \\
\hline 10.696034 & -1.083276 & 0.344159 \\
\hline 10.695805 & 1.082844 & -0.347790 \\
\hline 10.464917 & -1.199241 & -1.361610 \\
\hline
\end{tabular}

Table S22. The optimized Cartesian coordinates of the TSC-based ribbon fused via benzene-core linker with $\mathrm{n}=3$ (type II) in the ground singlet state calculated at the B3LYP/6-31(d) level of theory

$\begin{array}{llll}\text { Center } & \text { Atomic } & \text { Coordinates (Angstroms) } \\ \text { Number } & \text { Number } & \mathrm{X}\end{array}$




\begin{tabular}{|c|c|c|c|c|}
\hline 1 & 6 & 4.201167 & -8.285785 & 3.807053 \\
\hline 2 & 6 & 3.942459 & 1.274579 & -3.320174 \\
\hline 3 & 6 & 1.253153 & 12.324304 & -1.510853 \\
\hline 4 & 6 & -1.253153 & -12.324304 & -1.510853 \\
\hline 5 & 6 & -3.942459 & -1.274579 & -3.320174 \\
\hline 6 & 6 & -4.201167 & 8.285785 & 3.807053 \\
\hline 7 & 6 & 2.660715 & -14.101772 & 2.805741 \\
\hline 8 & 6 & 1.285494 & -3.941883 & -1.086559 \\
\hline 9 & 6 & -0.212318 & 6.484374 & -0.493167 \\
\hline 10 & 6 & 0.212318 & -6.484374 & -0.493167 \\
\hline 11 & 6 & -1.285494 & 3.941883 & -1.086559 \\
\hline 12 & 6 & -2.660715 & 14.101772 & 2.805741 \\
\hline 13 & 6 & -1.890253 & -11.113514 & -1.416315 \\
\hline 14 & 6 & -4.218706 & 0.038320 & -3.037695 \\
\hline 15 & 6 & -3.986000 & 9.429104 & 4.531431 \\
\hline 16 & 6 & 3.986000 & -9.429104 & 4.531431 \\
\hline 17 & 6 & 4.218706 & -0.038320 & -3.037695 \\
\hline 18 & 6 & 1.890253 & 11.113514 & -1.416315 \\
\hline 19 & 6 & 2.243251 & -14.517898 & 1.567421 \\
\hline 20 & 6 & -0.066266 & -4.238795 & -1.411035 \\
\hline 21 & 6 & -1.546042 & 6.164426 & -0.119579 \\
\hline 22 & 6 & 1.546042 & -6.164426 & -0.119579 \\
\hline 23 & 6 & 0.066266 & 4.238795 & -1.411035 \\
\hline 24 & 6 & -2.243251 & 14.517898 & 1.567421 \\
\hline 25 & 6 & 2.152309 & -11.713799 & 2.205932 \\
\hline 26 & 6 & 0.994375 & -1.576374 & -1.928327 \\
\hline 27 & 6 & -0.563355 & 8.868231 & 0.284928 \\
\hline 28 & 6 & 0.563355 & -8.868231 & 0.284928 \\
\hline 29 & 6 & -0.994375 & 1.576374 & -1.928327 \\
\hline 30 & 6 & -2.152309 & 11.713799 & 2.205932 \\
\hline 31 & 6 & 0.524741 & -11.544351 & 0.087666 \\
\hline 32 & 6 & -1.572877 & -0.982692 & -2.524525 \\
\hline 33 & 6 & -2.597988 & 9.073754 & 2.033839 \\
\hline 34 & 6 & 2.597988 & -9.073754 & 2.033839 \\
\hline 35 & 6 & 1.572877 & 0.982692 & -2.524525 \\
\hline 36 & 6 & -0.524741 & 11.544351 & 0.087666 \\
\hline 37 & 6 & 1.498067 & -12.182210 & 1.005705 \\
\hline 38 & 6 & -0.435751 & -1.844989 & -2.127229 \\
\hline 39 & 6 & -1.809769 & 8.449440 & 0.944893 \\
\hline 40 & 6 & 1.809769 & -8.449440 & 0.944893 \\
\hline 41 & 6 & 0.435751 & 1.844989 & -2.127229 \\
\hline 42 & 6 & -1.498067 & 12.182210 & 1.005705 \\
\hline 43 & 6 & 2.564356 & -10.369759 & 2.678545 \\
\hline 44 & 6 & 1.834740 & -0.427158 & -2.339244 \\
\hline 45 & 6 & 0.003572 & 10.201876 & -0.021374 \\
\hline 46 & 6 & -0.003572 & -10.201876 & -0.021374 \\
\hline 47 & 6 & -1.834740 & 0.427158 & -2.339244 \\
\hline 48 & 6 & -2.564356 & 10.369759 & 2.678545 \\
\hline 49 & 6 & 2.598647 & -12.738055 & 3.091444 \\
\hline 50 & 6 & 1.771461 & -2.673225 & -1.518757 \\
\hline 51 & 6 & 0.212318 & 7.827917 & -0.258836 \\
\hline 52 & 6 & -0.212318 & -7.827917 & -0.258836 \\
\hline 53 & 6 & -1.771461 & 2.673225 & -1.518757 \\
\hline 54 & 6 & -2.598647 & 12.738055 & 3.091444 \\
\hline 55 & 6 & -0.082089 & -12.504092 & -0.774199 \\
\hline 56 & 6 & -2.682641 & -1.759973 & -2.967058 \\
\hline 57 & 6 & -3.520506 & 8.144660 & 2.597737 \\
\hline 58 & 6 & 3.520506 & -8.144660 & 2.597737 \\
\hline 59 & 6 & 2.682641 & 1.759973 & -2.967058 \\
\hline 60 & 6 & 0.082089 & 12.504092 & -0.774199 \\
\hline 61 & 6 & 1.640264 & -13.574654 & 0.735230 \\
\hline 62 & 6 & -0.817402 & -3.196001 & -2.028951 \\
\hline 63 & 6 & -2.234752 & 7.139261 & 0.664710 \\
\hline 64 & 6 & 2.234752 & -7.139261 & 0.664710 \\
\hline 65 & 6 & 0.817402 & 3.196001 & -2.028951 \\
\hline 66 & 6 & -1.640264 & 13.574654 & 0.735230 \\
\hline 67 & 6 & 3.230272 & -10.440810 & 3.936946 \\
\hline 68 & 6 & 3.197940 & -0.816812 & -2.491515 \\
\hline 69 & 6 & 1.236103 & 10.091815 & -0.728101 \\
\hline 70 & 6 & -1.236103 & -10.091815 & -0.728101 \\
\hline 71 & 6 & -3.197940 & 0.816812 & -2.491515 \\
\hline 72 & 6 & -3.230272 & 10.440810 & 3.936946 \\
\hline 73 & 6 & -0.573623 & -5.508419 & -1.116854 \\
\hline 74 & 6 & -2.064481 & 4.905395 & -0.440447 \\
\hline 75 & 6 & 2.064481 & -4.905395 & -0.440447 \\
\hline 76 & 6 & 0.573623 & 5.508419 & -1.116854 \\
\hline 77 & 34 & 3.702996 & -6.552554 & 1.653018 \\
\hline 78 & 34 & 2.459002 & 3.604436 & -2.810198 \\
\hline
\end{tabular}




\begin{tabular}{|c|c|c|}
\hline-0.721275 & 14.189652 & -0.770478 \\
\hline 0.721275 & -14.189652 & -0.770478 \\
\hline-2.459002 & -3.604436 & -2.810198 \\
\hline-3.702996 & 6.552554 & 1.653018 \\
\hline 3.268553 & -12.133692 & 4.725035 \\
\hline 3.602633 & -2.505376 & -1.818920 \\
\hline 1.877315 & 8.352529 & -0.917612 \\
\hline-1.877315 & -8.352529 & -0.917612 \\
\hline-3.602633 & 2.505376 & -1.818920 \\
\hline-3.268553 & 12.133692 & 4.725035 \\
\hline-1.605924 & -5.734681 & -1.370737 \\
\hline-3.086647 & 4.657350 & -0.163199 \\
\hline 3.086647 & -4.657350 & -0.163199 \\
\hline 1.605924 & 5.734681 & -1.370737 \\
\hline 4.849470 & -7.494886 & 4.171037 \\
\hline 4.706767 & 1.945412 & -3.700294 \\
\hline 1.661923 & 13.137314 & -2.102378 \\
\hline 4.456647 & -9.587685 & 5.496546 \\
\hline 5.211029 & -0.453509 & -3.184470 \\
\hline 2.826781 & 10.922151 & -1.930632 \\
\hline-1.661923 & -13.137314 & -2.102378 \\
\hline-4.706767 & -1.945412 & -3.700294 \\
\hline-4.849470 & 7.494886 & 4.171037 \\
\hline-2.826781 & -10.922151 & -1.930632 \\
\hline-5.211029 & 0.453509 & -3.184470 \\
\hline-4.456647 & 9.587685 & 5.496546 \\
\hline-2.297154 & 15.560560 & 1.270517 \\
\hline-3.062061 & 14.799101 & 3.534308 \\
\hline 3.062061 & -14.799101 & 3.534308 \\
\hline 2.297154 & -15.560560 & 1.270517 \\
\hline
\end{tabular}

Table S23. The optimized Cartesian coordinates of the TSC-based ribbon fused via benzene-core linker with $\mathrm{n}=4$ (type II) in the ground singlet state calculated at the B3LYP/6-31(d) level of theory

\begin{tabular}{|c|c|c|c|c|}
\hline \multirow{2}{*}{$\begin{array}{l}\text { Center } \\
\text { Number }\end{array}$} & \multirow{2}{*}{$\begin{array}{l}\text { Atomic } \\
\text { Number }\end{array}$} & \multicolumn{3}{|c|}{ Coordinates (Angstroms) } \\
\hline & & $\mathrm{X}$ & $\mathrm{Y}$ & Z \\
\hline 1 & 6 & -15.705896 & 3.705219 & -1.331528 \\
\hline 2 & 6 & -4.232657 & 4.234869 & -3.057299 \\
\hline 3 & 6 & 5.593315 & 4.222471 & 3.195405 \\
\hline 4 & 6 & 17.036136 & 3.399995 & 1.316941 \\
\hline 5 & 6 & -15.223711 & -3.768621 & 2.932531 \\
\hline 6 & 6 & -5.497471 & -3.967975 & -3.446665 \\
\hline 7 & 6 & 4.139990 & -3.978742 & 3.230417 \\
\hline 8 & 6 & 14.323619 & -4.045929 & -1.929797 \\
\hline 9 & 6 & -19.598606 & 0.368010 & 1.972408 \\
\hline 10 & 6 & -9.204565 & 0.745097 & -1.584486 \\
\hline 11 & 6 & 1.037860 & 0.815411 & 0.635760 \\
\hline 12 & 6 & 11.524333 & 0.683542 & 0.835852 \\
\hline 13 & 6 & -11.498751 & -0.736368 & -0.860095 \\
\hline 14 & 6 & -1.038331 & -0.603925 & -0.643637 \\
\hline 15 & 6 & 9.191543 & -0.677567 & 1.651567 \\
\hline 16 & 6 & 19.137261 & -0.421191 & -2.986091 \\
\hline 17 & 6 & -14.312258 & -4.042424 & 1.945640 \\
\hline 18 & 6 & -4.140067 & -3.966287 & -3.255046 \\
\hline 19 & 6 & 5.496361 & -3.978480 & 3.429205 \\
\hline 20 & 6 & 15.234540 & -3.772845 & -2.917375 \\
\hline 21 & 6 & -17.040178 & 3.392929 & -1.315183 \\
\hline 22 & 6 & -5.597216 & 4.232308 & -3.188090 \\
\hline 23 & 6 & 4.228252 & 4.223162 & 3.069988 \\
\hline 24 & 6 & 15.701280 & 3.709728 & 1.334152 \\
\hline 25 & 6 & -19.136922 & -0.429059 & 2.988223 \\
\hline 26 & 6 & -9.191412 & -0.674345 & -1.652003 \\
\hline 27 & 6 & 1.038940 & -0.606685 & 0.630444 \\
\hline 28 & 6 & 11.500093 & -0.737381 & 0.862963 \\
\hline 29 & 6 & -11.525000 & 0.684565 & -0.834999 \\
\hline 30 & 6 & -1.040817 & 0.818166 & -0.636809 \\
\hline 31 & 6 & 9.202854 & 0.741799 & 1.582198 \\
\hline 32 & 6 & 19.597777 & 0.379236 & -1.972395 \\
\hline 33 & 6 & -17.307864 & 0.421278 & 0.936375 \\
\hline 34 & 6 & -6.743485 & 0.818239 & -2.152727 \\
\hline
\end{tabular}




\begin{tabular}{|c|c|c|c|c|}
\hline 35 & 6 & 3.299280 & 0.835765 & 1.761884 \\
\hline 36 & 6 & 13.955398 & 0.640740 & 0.119376 \\
\hline 37 & 6 & -13.817788 & -0.799827 & 0.159374 \\
\hline 38 & 6 & -3.292904 & -0.625631 & -1.784031 \\
\hline 39 & 6 & 6.727963 & -0.654650 & 2.210591 \\
\hline 40 & 6 & 16.895266 & -0.601799 & -1.859950 \\
\hline 41 & 6 & -15.751778 & -1.551480 & 1.867179 \\
\hline 42 & 6 & -5.662511 & -1.617775 & -2.564314 \\
\hline 43 & 6 & 4.229355 & -1.620832 & 2.358689 \\
\hline 44 & 6 & 14.619240 & -1.732129 & -0.982209 \\
\hline 45 & 6 & -15.150153 & 1.508688 & -0.234166 \\
\hline 46 & 6 & -4.263579 & 1.837078 & -2.296403 \\
\hline 47 & 6 & 5.698578 & 1.823319 & 2.446384 \\
\hline 48 & 6 & 16.562467 & 1.308882 & -0.002566 \\
\hline 49 & 6 & -16.893275 & -0.605652 & 1.864819 \\
\hline 50 & 6 & -6.728836 & -0.648826 & -2.215372 \\
\hline 51 & 6 & 3.292704 & -0.632503 & 1.772484 \\
\hline 52 & 6 & 13.820332 & -0.798943 & -0.153844 \\
\hline 53 & 6 & -13.955274 & 0.639191 & -0.116114 \\
\hline 54 & 6 & -3.301874 & 0.842521 & -1.763625 \\
\hline 55 & 6 & 6.741297 & 0.812472 & 2.148938 \\
\hline 56 & 6 & 17.308054 & 0.427986 & -0.933866 \\
\hline 57 & 6 & -16.563383 & 1.303029 & 0.005045 \\
\hline 58 & 6 & -5.701373 & 1.830561 & -2.447218 \\
\hline 59 & 6 & 4.260441 & 1.828668 & 2.298714 \\
\hline 60 & 6 & 15.148975 & 1.512083 & 0.237251 \\
\hline 61 & 6 & -14.614166 & -1.732445 & 0.990798 \\
\hline 62 & 6 & -4.229557 & -1.612044 & -2.373431 \\
\hline 63 & 6 & 5.661635 & -1.625341 & 2.554649 \\
\hline 64 & 6 & 15.756438 & -1.550867 & -1.859065 \\
\hline 65 & 6 & -18.689304 & 0.769317 & 0.994098 \\
\hline 66 & 6 & -8.009584 & 1.408521 & -1.988633 \\
\hline 67 & 6 & 2.103419 & 1.446052 & 1.341809 \\
\hline 68 & 6 & 12.779822 & 1.292531 & 0.531351 \\
\hline 69 & 6 & -12.650826 & -1.404293 & -0.343264 \\
\hline 70 & 6 & -2.097223 & -1.233435 & -1.360561 \\
\hline 71 & 6 & 7.984750 & -1.278447 & 2.114114 \\
\hline 72 & 6 & 17.835214 & -0.911168 & -2.886529 \\
\hline 73 & 6 & -15.912517 & -2.557329 & 2.864804 \\
\hline 74 & 6 & -6.215580 & -2.854262 & -3.009821 \\
\hline 75 & 6 & 3.572600 & -2.858942 & 2.621383 \\
\hline 76 & 6 & 14.020046 & -3.024514 & -1.02997 \\
\hline 77 & 6 & -14.814092 & 2.770733 & -0.805500 \\
\hline 78 & 6 & -3.629508 & 3.092761 & -2.529297 \\
\hline 79 & 6 & 6.281839 & 3.077263 & 2.793889 \\
\hline 80 & 6 & 17.427060 & 2.251156 & 0.628000 \\
\hline 81 & 6 & -17.833250 & -0.915234 & 2.891288 \\
\hline 82 & 6 & -7.984692 & -1.274035 & -2.116298 \\
\hline 83 & 6 & 2.098744 & -1.239514 & 1.343110 \\
\hline 84 & 6 & 12.654009 & -1.404395 & 0.34911 \\
\hline 85 & 6 & -12.780878 & 1.292240 & -0.52946 \\
\hline 86 & 6 & -2.107504 & 1.452022 & -1.338282 \\
\hline 87 & 6 & 8.006790 & 1.404070 & 1.985043 \\
\hline 88 & 6 & 18.688544 & 0.779483 & -0.99360 \\
\hline 89 & 6 & -17.429371 & 2.243288 & -0.62661 \\
\hline 90 & 6 & -6.285337 & 3.084752 & -2.79261 \\
\hline 91 & 6 & 3.625132 & 3.082262 & 2.53933 \\
\hline 92 & 6 & 14.810941 & 2.773700 & 0.808383 \\
\hline 93 & 6 & -14.011798 & -3.023208 & 1.042291 \\
\hline 94 & 6 & -3.571834 & -2.847889 & -2.64420 \\
\hline 95 & 6 & 6.214626 & -2.862168 & 2.99929 \\
\hline 96 & 6 & 15.919779 & -2.559313 & -2.85364 \\
\hline 97 & 6 & -10.334655 & -1.389272 & -1.28286 \\
\hline 98 & 6 & 0.001205 & -1.292173 & -0.00953 \\
\hline 99 & 6 & 10.336308 & -1.391366 & 1.28490 \\
\hline 100 & 6 & -10.371809 & 1.398163 & -1.17910 \\
\hline 01 & 6 & -0.002367 & 1.503534 & 0.00238 \\
\hline 02 & 6 & 10.369657 & 1.395975 & 1.17735 \\
\hline 03 & 34 & -12.989151 & 3.089871 & -0.98024 \\
\hline 104 & 34 & -1.886906 & 3.219604 & -1.88203 \\
\hline 105 & 34 & 8.120405 & 3.186144 & 2.514720 \\
\hline 106 & 34 & 19.248388 & 2.010716 & 0.29258 \\
\hline 07 & 34 & -17.302739 & -2.258676 & 4.07513 \\
\hline 108 & 34 & -8.060626 & -3.005926 & -2.795863 \\
\hline 109 & 34 & 1.851413 & -2.992624 & 1.92145 \\
\hline 10 & 34 & 12.553630 & -3.249927 & 0.09551 \\
\hline 11 & 34 & -19.250552 & 1.998421 & -0.293581 \\
\hline 12 & 34 & -8.124651 & 3.190859 & -2.51704 \\
\hline 13 & 34 & 1.880615 & 3.209710 & 1.897305 \\
\hline
\end{tabular}




\section{1}

2

3

4

5

\section{6}

7

8

10

11

12

13

$\begin{array}{rrr}12.985522 & 3.090406 & 0.982267 \\ -12.545996 & -3.248798 & -0.083935 \\ -1.847945 & -2.982236 & -1.950943 \\ 8.060735 & -3.010845 & 2.792383 \\ 17.308100 & -2.259842 & -4.065921 \\ -10.291315 & -2.475590 & -1.273169 \\ 0.002490 & -2.379981 & -0.013991 \\ 10.294402 & -2.477744 & 1.276131 \\ -10.372017 & 2.483363 & -1.109052 \\ -0.003684 & 2.591234 & 0.006845 \\ 10.368396 & 2.481078 & 1.105736 \\ -15.341823 & 4.623975 & -1.780496 \\ -3.644809 & 5.123308 & -3.266799 \\ 6.133770 & 5.108474 & 3.514260 \\ 17.779065 & 4.061652 & 1.750896 \\ -17.784066 & 4.053098 & -1.749759 \\ -6.137939 & 5.118596 & -3.505697 \\ 3.639710 & 5.109782 & 3.285167 \\ 15.335741 & 4.627849 & 1.783226 \\ -15.448795 & -4.487372 & 3.713994 \\ -6.013574 & -4.839396 & -3.837863 \\ 3.534054 & -4.850683 & 3.456976 \\ 13.794064 & -4.992613 & -1.890435 \\ -13.780216 & -4.987828 & 1.909179 \\ -3.533671 & -4.836275 & -3.487800 \\ 6.012079 & -4.850252 & 3.820128 \\ 15.461700 & -4.493472 & -3.696506 \\ 20.623267 & 0.734141 & -1.949557 \\ 19.781699 & -0.731182 & -3.802642 \\ -20.625062 & 0.719994 & 1.947791 \\ -19.781343 & -0.739165 & 3.804744\end{array}$

Table S24. The optimized Cartesian coordinates of the TSC-based ribbon fused via benzene-core linker with $\mathrm{n}=5$ (type II) in the ground singlet state calculated at the B3LYP/6-31(d) level of theory

\begin{tabular}{|c|c|c|c|c|}
\hline \multirow{2}{*}{$\begin{array}{l}\text { Center } \\
\text { Number }\end{array}$} & \multirow{2}{*}{$\begin{array}{l}\text { Atomic } \\
\text { Number }\end{array}$} & \multicolumn{3}{|c|}{ Coordinates (Angstroms) } \\
\hline & & $\mathrm{x}$ & $\mathrm{Y}$ & Z \\
\hline 1 & 6 & 1.575578 & 21.197887 & -1.777689 \\
\hline 2 & 6 & 2.408043 & 9.890044 & -3.698986 \\
\hline 3 & 6 & 4.145092 & -0.088462 & 2.386108 \\
\hline 4 & 6 & 5.745577 & -10.674168 & -1.867723 \\
\hline 5 & 6 & 3.440326 & -20.228632 & 5.048663 \\
\hline 6 & 6 & -3.440326 & 20.228632 & 5.048663 \\
\hline 7 & 6 & -5.745577 & 10.674168 & -1.867723 \\
\hline 8 & 6 & -4.145092 & 0.088462 & 2.386108 \\
\hline 9 & 6 & -2.408043 & -9.890044 & -3.698986 \\
\hline 10 & 6 & -1.575578 & -21.197887 & -1.777689 \\
\hline 11 & 6 & -1.713384 & 25.057812 & 1.667965 \\
\hline 12 & 6 & -0.891740 & 14.547195 & -1.271541 \\
\hline 13 & 6 & 0.110161 & 4.152920 & 0.179331 \\
\hline 14 & 6 & 1.637042 & -6.168215 & -0.744097 \\
\hline 15 & 6 & 2.085606 & -16.646415 & 0.191344 \\
\hline 16 & 6 & -2.085606 & 16.646415 & 0.191344 \\
\hline 17 & 6 & -1.637042 & 6.168215 & -0.744097 \\
\hline 18 & 6 & -0.110161 & -4.152920 & 0.179331 \\
\hline 19 & 6 & 0.891740 & -14.547195 & -1.271541 \\
\hline 20 & 6 & 1.713384 & -25.057812 & 1.667965 \\
\hline 21 & 6 & -3.872390 & 19.096813 & 4.407965 \\
\hline 22 & 6 & -5.631474 & 9.319192 & -2.042942 \\
\hline 23 & 6 & -3.971330 & -1.269150 & 2.307882 \\
\hline 24 & 6 & -2.205590 & -11.228971 & -3.912895 \\
\hline 25 & 6 & -1.051042 & -22.464445 & -1.817458 \\
\hline 26 & 6 & 1.051042 & 22.464445 & -1.817458 \\
\hline 27 & 6 & 2.205590 & 11.228971 & -3.912895 \\
\hline 28 & 6 & 3.971330 & 1.269150 & 2.307882 \\
\hline 29 & 6 & 5.631474 & -9.319192 & -2.042942 \\
\hline 30 & 6 & 3.872390 & -19.096813 & 4.407965 \\
\hline 31 & 6 & -1.973101 & 24.711579 & 2.969328 \\
\hline 32 & 6 & -2.196122 & 14.391524 & -0.728546 \\
\hline 33 & 6 & -1.298179 & 3.976109 & 0.268397 \\
\hline
\end{tabular}


$-0.223630$

0.621078

2.147178

2. 767032

1. 370379

1. 902603

3. 488582

4.721816

2. 455471

$-2.455471$

$-4.721816$

$-3.488582$

$-1.902603$

$-1.370379$

$-0.602052$

1.117876

2. 651932

4.247962

3. 887141

$-3.887141$

$-4.247962$

$-2.651932$

$-1.117876$

0.602052

$-3.750298$

$-3.223995$

$-1.698390$

$-0.771190$

0.771190

1. 698390

3. 223995

3. 750298

2. 399754

3. 288210

5.086985

6.713233

3. 736722

1. 441022

2. 918679

4. 769393

6.505060

4. 526553

$-3.736722$

$-6.713233$

$-5.086985$

$-3.288210$

$-2.399754$

$-4.526553$

$-6.505060$

$-4.769393$

$-2.918679$

$-1.441022$

$-1.714244$

$-2.190745$

2. 190745

1. 741407

$-8.751642$

$-18.864715$

18.864715

8.751642

$-1.741407$

$-11.829574$

$-22.775091$

15.440092

4.987909

$-5.330139$

$-15.763774$

15.763774

5.330139

$-4.987909$

$-15.440092$

18.461984

7.502201

$-2.685750$

$-13.156134$

$-22.854673$

22.854673

13.156134

2.685750

$-7.502201$

$-18.461984$

24.531520

13.684286

3.523410

$-6.917937$

$-17.280683$

17.280683

6.917937

$-3.523410$

$-13.684286$

$-24.531520$

15.299673

4.855555

$-5.461772$

$-15.880986$

15.880986

5.461772

$-4.855555$

$-15.299673$

20.903444

9.381519

$-0.521543$

$-11.166450$

$-20.452894$

23.220526

11.827886

1.958583

$-8.690997$

$-18.381081$

20.452894

11.166450

0.521543

$-9.381519$

$-20.903444$

18.381081

8.690997

$-1.958583$

$-11.827886$

$-23.220526$

26.094070

25.460555

$-25.460555$

1.784940

$-1.890360$

3.113633

3. 113633

$-1.890360$

1. 784940

$-3.271978$

$-0.920805$

$-0.002158$

$-0.192166$

$-0.376709$

$-1.101886$

$-1.101886$

$-0.376709$

$-0.192166$

$-0.002158$

$-1.190562$

$-2.290070$

1. 527808

$-1.008514$

5.029941

5.029941

$-1.008514$

1. 527808

$-2.290070$

$-1.190562$

$-0.857443$

$-3.085024$

1. 254391

$-1.575118$

2. 264523

2. 264523

$-1.575118$

1. 254391

$-3.085024$

$-0.857443$

0.441284

$-0.116586$

$-0.451907$

$-1.523824$

$-1.523824$

$-0.451907$

$-0.116586$

0.441284

$-2.419766$

$-4.080306$

2. 708704

$-1.867820$

6.068446

$-2.491427$

$-4.471054$

2. 565845

$-2.187713$

4.895922

6.068446

$-1.867820$

2. 708704

$-4.080306$

$-2.419766$

4.895922

$-2.187713$

2. 565845

$-4.471054$

$-2.491427$

1. 344896

3. 724195

3. 724195

1. 344896

Table S25. The optimized Cartesian coordinates of the TSC-based ribbon fused through four-membered ring with $\mathrm{n}=2$ (type III) in the ground singlet state calculated at the B3LYP/6-31(d) level of theory 


\begin{tabular}{|c|c|c|c|c|}
\hline \multirow{2}{*}{$\begin{array}{l}\text { Center } \\
\text { Number }\end{array}$} & \multirow{2}{*}{$\begin{array}{l}\text { Atomic } \\
\text { Number }\end{array}$} & \multicolumn{3}{|c|}{ Coordinates (Angstroms) } \\
\hline & & $\mathrm{x}$ & $\mathrm{Y}$ & $\mathrm{Z}$ \\
\hline 1 & 6 & -4.345848 & 4.004056 & -1.484634 \\
\hline 2 & 6 & 5.577755 & 3.754418 & 2.031595 \\
\hline 3 & 6 & -5.577755 & -3.754419 & 2.031595 \\
\hline 4 & 6 & 4.345848 & -4.004057 & -1.484635 \\
\hline 5 & 6 & -9.216397 & 0.440119 & -0.524704 \\
\hline 6 & 6 & 0.749794 & 0.697837 & -0.001354 \\
\hline 7 & 6 & -0.749794 & -0.697837 & -0.001354 \\
\hline 8 & 6 & 9.216397 & -0.440121 & -0.524702 \\
\hline 9 & 6 & -4.345852 & -4.004064 & 1.484618 \\
\hline 10 & 6 & 5.577752 & -3.754411 & -2.031609 \\
\hline 11 & 6 & -5.577752 & 3.754410 & -2.031608 \\
\hline 12 & 6 & 4.345852 & 4.004064 & 1.484618 \\
\hline 13 & 6 & -9.216397 & -0.440109 & 0.524722 \\
\hline 14 & 6 & 0.749795 & -0.697836 & 0.001365 \\
\hline 15 & 6 & -0.749795 & 0.697836 & 0.001365 \\
\hline 16 & 6 & 9.216396 & 0.440108 & 0.524724 \\
\hline 17 & 6 & -6.703099 & 0.553771 & -0.464655 \\
\hline 18 & 6 & 3.211531 & 0.714178 & 0.101197 \\
\hline 19 & 6 & -3.211531 & -0.714178 & 0.101196 \\
\hline 20 & 6 & 6.703099 & -0.553771 & -0.464654 \\
\hline 21 & 6 & -5.653182 & -1.511023 & 0.896100 \\
\hline 22 & 6 & 4.262023 & -1.674886 & -0.533243 \\
\hline 23 & 6 & -4.262023 & 1.674885 & -0.533242 \\
\hline 24 & 6 & 5.653182 & 1.511023 & 0.896101 \\
\hline 25 & 6 & -6.703099 & -0.553769 & 0.464659 \\
\hline 26 & 6 & 3.211532 & -0.714176 & -0.101196 \\
\hline 27 & 6 & -3.211532 & 0.714176 & -0.101196 \\
\hline 28 & 6 & 6.703099 & 0.553769 & 0.464660 \\
\hline 29 & 6 & -5.653181 & 1.511020 & -0.896102 \\
\hline 30 & 6 & 4.262024 & 1.674888 & 0.533238 \\
\hline 31 & 6 & -4.262024 & -1.674889 & 0.533238 \\
\hline 32 & 6 & 5.653181 & -1.511020 & -0.896102 \\
\hline 33 & 6 & -7.983440 & 0.921810 & -0.969513 \\
\hline 34 & 6 & 1.924321 & 1.385031 & 0.054351 \\
\hline 35 & 6 & -1.924321 & -1.385031 & 0.054350 \\
\hline 36 & 6 & 7.983440 & -0.921811 & -0.969512 \\
\hline 37 & 6 & -6.197879 & -2.547578 & 1.708560 \\
\hline 38 & 6 & 3.724242 & -2.974428 & -0.778610 \\
\hline 39 & 6 & -3.724242 & 2.974428 & -0.778609 \\
\hline 40 & 6 & 6.197879 & 2.547578 & 1.708561 \\
\hline 41 & 6 & -7.983438 & -0.921806 & 0.969523 \\
\hline 42 & 6 & 1.924321 & -1.385029 & -0.054346 \\
\hline 43 & 6 & -1.924321 & 1.385029 & -0.054346 \\
\hline 44 & 6 & 7.983437 & 0.921805 & 0.969525 \\
\hline 45 & 6 & -6.197878 & 2.547573 & -1.708565 \\
\hline 46 & 6 & 3.724245 & 2.974433 & 0.778599 \\
\hline 47 & 6 & -3.724245 & -2.974433 & 0.778600 \\
\hline 48 & 6 & 6.197878 & -2.547573 & -1.708565 \\
\hline 49 & 34 & -1.947531 & 3.237232 & -0.236417 \\
\hline 50 & 34 & 7.979051 & 2.312122 & 2.213923 \\
\hline 51 & 34 & -7.979051 & -2.312123 & 2.213922 \\
\hline 52 & 34 & 1.947531 & -3.237231 & -0.236418 \\
\hline 53 & 34 & -7.979054 & 2.312122 & -2.213917 \\
\hline 54 & 34 & 1.947530 & 3.237234 & 0.236412 \\
\hline 55 & 34 & -1.947530 & -3.237234 & 0.236412 \\
\hline 56 & 34 & 7.979055 & -2.312123 & -2.213916 \\
\hline 57 & 1 & -3.835436 & 4.948806 & -1.642558 \\
\hline 58 & 1 & 6.089251 & 4.491399 & 2.642549 \\
\hline 59 & 1 & -6.089249 & 4.491389 & -2.642565 \\
\hline 60 & 1 & 3.835442 & 4.948815 & 1.642537 \\
\hline 61 & 1 & -3.835442 & -4.948814 & 1.642538 \\
\hline 62 & 1 & 6.089249 & -4.491389 & -2.642566 \\
\hline 63 & 1 & -6.089251 & -4.491399 & 2.642550 \\
\hline 64 & 1 & 3.835436 & -4.948805 & -1.642560 \\
\hline 65 & 1 & 10.140343 & 0.806415 & 0.960875 \\
\hline 66 & 1 & 10.140346 & -0.806432 & -0.960848 \\
\hline 67 & 1 & -10.140346 & 0.806431 & -0.960850 \\
\hline 68 & 1 & -10.140343 & -0.806416 & 0.960873 \\
\hline
\end{tabular}


Table S26. The optimized Cartesian coordinates of the TSC-based ribbon fused through four-membered ring with $\mathrm{n}=3$ (type III) in the ground singlet state calculated at the B3LYP/6-31(d) level of theory

\begin{tabular}{|c|c|c|c|c|}
\hline \multirow{2}{*}{$\begin{array}{l}\text { Center } \\
\text { Number }\end{array}$} & \multirow{2}{*}{$\begin{array}{l}\text { Atomic } \\
\text { Number }\end{array}$} & \multicolumn{3}{|c|}{ Coordinates (Angstroms) } \\
\hline & & $\mathrm{X}$ & $\mathrm{Y}$ & Z \\
\hline 1 & 6 & 0.373353 & -9.307816 & 4.254056 \\
\hline 2 & 6 & -0.314884 & 0.608926 & 4.253099 \\
\hline 3 & 6 & 0.227799 & 10.539348 & 4.262332 \\
\hline 4 & 6 & 0.227799 & -10.539349 & -4.262331 \\
\hline 5 & 6 & -0.314884 & -0.608926 & -4.253099 \\
\hline 6 & 6 & 0.373355 & 9.307817 & -4.254056 \\
\hline 7 & 6 & -0.286379 & -14.176179 & 0.622085 \\
\hline 8 & 6 & 0.305420 & -4.205308 & 0.627265 \\
\hline 9 & 6 & -0.305574 & 5.706558 & 0.627062 \\
\hline 10 & 6 & -0.305573 & -5.706558 & -0.627063 \\
\hline 11 & 6 & 0.305419 & 4.205308 & -0.627266 \\
\hline 12 & 6 & -0.286385 & 14.176179 & -0.622085 \\
\hline 13 & 6 & -0.373355 & -9.307817 & -4.254056 \\
\hline 14 & 6 & 0.314884 & 0.608926 & -4.253099 \\
\hline 15 & 6 & -0.227799 & 10.539349 & -4.262331 \\
\hline 16 & 6 & -0.227799 & -10.539348 & 4.262332 \\
\hline 17 & 6 & 0.314884 & -0.608926 & 4.253099 \\
\hline 18 & 6 & -0.373353 & 9.307816 & 4.254056 \\
\hline 19 & 6 & 0.286385 & -14.176179 & -0.622085 \\
\hline 20 & 6 & -0.305419 & -4.205308 & -0.627266 \\
\hline 21 & 6 & 0.305573 & 5.706558 & -0.627063 \\
\hline 22 & 6 & 0.305574 & -5.706558 & 0.627062 \\
\hline 23 & 6 & -0.305420 & 4.205308 & 0.627265 \\
\hline 24 & 6 & 0.286379 & 14.176179 & 0.622085 \\
\hline 25 & 6 & -0.183395 & -11.662765 & 0.699145 \\
\hline 26 & 6 & 0.208635 & -1.744673 & 0.689863 \\
\hline 27 & 6 & -0.212869 & 8.168842 & 0.689436 \\
\hline 28 & 6 & -0.212868 & -8.168842 & -0.689436 \\
\hline 29 & 6 & 0.208634 & 1.744673 & -0.689863 \\
\hline 30 & 6 & -0.183399 & 11.662765 & -0.699144 \\
\hline 31 & 6 & 0.164306 & -10.612601 & -1.748433 \\
\hline 32 & 6 & -0.209754 & -0.692886 & -1.740979 \\
\hline 33 & 6 & 0.233175 & 9.220706 & -1.741414 \\
\hline 34 & 6 & 0.233174 & -9.220706 & 1.741414 \\
\hline 35 & 6 & -0.209755 & 0.692886 & 1.740978 \\
\hline 36 & 6 & 0.164304 & 10.612601 & 1.748433 \\
\hline 37 & 6 & 0.183399 & -11.662765 & -0.699144 \\
\hline 38 & 6 & -0.208634 & -1.744673 & -0.689863 \\
\hline 39 & 6 & 0.212868 & 8.168842 & -0.689436 \\
\hline 40 & 6 & 0.212869 & -8.168842 & 0.689436 \\
\hline 41 & 6 & -0.208635 & 1.744673 & 0.689863 \\
\hline 42 & 6 & 0.183395 & 11.662765 & 0.699145 \\
\hline 43 & 6 & -0.164304 & -10.612601 & 1.748433 \\
\hline 44 & 6 & 0.209755 & -0.692886 & 1.740978 \\
\hline 45 & 6 & -0.233174 & 9.220706 & 1.741414 \\
\hline 46 & 6 & -0.233175 & -9.220706 & -1.741414 \\
\hline 47 & 6 & 0.209754 & 0.692886 & -1.740979 \\
\hline 48 & 6 & -0.164306 & 10.612601 & -1.748433 \\
\hline 49 & 6 & -0.482546 & -12.943195 & 1.247582 \\
\hline 50 & 6 & 0.540218 & -3.029825 & 1.277170 \\
\hline 51 & 6 & -0.542845 & 6.881404 & 1.275556 \\
\hline 52 & 6 & -0.542845 & -6.881404 & -1.275556 \\
\hline 53 & 6 & 0.540218 & 3.029825 & -1.277171 \\
\hline 54 & 6 & -0.482551 & 12.943195 & -1.247581 \\
\hline 55 & 6 & 0.454386 & -11.157918 & -3.032831 \\
\hline 56 & 6 & -0.537821 & -1.226410 & -3.022850 \\
\hline 57 & 6 & 0.568321 & 8.684924 & -3.021194 \\
\hline 58 & 6 & 0.568320 & -8.684923 & 3.021194 \\
\hline 59 & 6 & -0.537821 & 1.226410 & 3.022849 \\
\hline 60 & 6 & 0.454385 & 11.157918 & 3.032831 \\
\hline 61 & 6 & 0.482551 & -12.943195 & -1.247581 \\
\hline 62 & 6 & -0.540218 & -3.029825 & -1.277171 \\
\hline 63 & 6 & 0.542845 & 6.881404 & -1.275556 \\
\hline 64 & 6 & 0.542845 & -6.881404 & 1.275556 \\
\hline 65 & 6 & -0.540218 & 3.029825 & 1.277170 \\
\hline 66 & 6 & 0.482546 & 12.943195 & 1.247582 \\
\hline 67 & 6 & -0.454385 & -11.157918 & 3.032831 \\
\hline 68 & 6 & 0.537821 & -1.226410 & 3.022849 \\
\hline
\end{tabular}




$\begin{array}{rrr}-0.568320 & 8.684923 & 3.021194 \\ -0.568321 & -8.684924 & -3.021194 \\ 0.537821 & 1.226410 & -3.022850 \\ -0.454386 & 11.157918 & -3.032831 \\ 1.153053 & -6.903799 & 3.033807 \\ -1.142670 & 3.001547 & 3.038495 \\ 1.012329 & 12.938843 & 3.036453 \\ 1.012333 & -12.938842 & -3.036453 \\ -1.142669 & -3.001548 & -3.038495 \\ 1.153054 & 6.903800 & -3.033806 \\ -1.012329 & -12.938843 & 3.036453 \\ 1.142670 & -3.001547 & 3.038495 \\ -1.153053 & 6.903799 & 3.033807 \\ -1.153054 & -6.903800 & -3.033806 \\ 1.142669 & 3.001548 & -3.038495 \\ -1.012333 & 12.938842 & -3.036453 \\ 0.635739 & -8.797687 & 5.175448 \\ -0.575070 & 1.113465 & 5.178123 \\ 0.463141 & 11.051921 & 5.189638 \\ -0.463141 & -11.051921 & 5.189638 \\ 0.575070 & -1.113465 & 5.178123 \\ -0.635739 & 8.797687 & 5.175448 \\ -0.635742 & -8.797689 & -5.175447 \\ 0.575071 & 1.113465 & -5.178123 \\ -0.463141 & 11.051921 & -5.189638 \\ 0.463141 & -11.051921 & -5.189638 \\ -0.575071 & -1.113465 & -5.178123 \\ 0.635742 & 8.797689 & -5.175447 \\ 0.524165 & 15.100094 & 1.139691 \\ -0.524173 & 15.100094 & -1.139690 \\ -0.524165 & -15.100094 & 1.139691 \\ 0.524173 & -15.100094 & -1.139690 \\ --------------------------------\end{array}$

Table S27. The optimized Cartesian coordinates of the TSC-based ribbon fused through four-membered ring with $\mathrm{n}=4$ (type III) in the ground singlet state calculated at the B3LYP/6-31(d) level of theory

\begin{tabular}{|c|c|c|c|c|}
\hline \multirow{2}{*}{$\begin{array}{l}\text { Center } \\
\text { Number }\end{array}$} & \multirow{2}{*}{$\begin{array}{l}\text { Atomic } \\
\text { Number }\end{array}$} & \multicolumn{3}{|c|}{ Coordinates (Angstroms) } \\
\hline & & $\mathrm{x}$ & Y & Z \\
\hline 1 & 6 & -14.259973 & 3.691241 & 2.147505 \\
\hline 2 & 6 & -4.340341 & 3.981084 & 1.530319 \\
\hline 3 & 6 & 5.558985 & 3.713026 & -2.099278 \\
\hline 4 & 6 & 15.491670 & 3.954296 & -1.607272 \\
\hline 5 & 6 & -15.491669 & -3.954300 & -1.607269 \\
\hline 6 & 6 & -5.558983 & -3.713021 & -2.099285 \\
\hline 7 & 6 & 4.340339 & -3.981079 & 1.530326 \\
\hline 8 & 6 & 14.259972 & -3.691245 & 2.147503 \\
\hline 9 & 6 & -19.128509 & 0.684809 & 0.005631 \\
\hline 10 & 6 & -9.157472 & 0.437983 & 0.543010 \\
\hline 11 & 6 & 0.749806 & 0.697903 & 0.001335 \\
\hline 12 & 6 & 10.658762 & 0.437683 & -0.543145 \\
\hline 13 & 6 & -10.658762 & -0.437683 & -0.543146 \\
\hline 14 & 6 & -0.749806 & -0.697900 & 0.001334 \\
\hline 15 & 6 & 9.157472 & -0.437983 & 0.543012 \\
\hline 16 & 6 & 19.128509 & -0.684814 & 0.005630 \\
\hline 17 & 6 & -14.259973 & -3.691245 & -2.147497 \\
\hline 18 & 6 & -4.340340 & -3.981078 & -1.530333 \\
\hline 19 & 6 & 5.558982 & -3.713024 & 2.099279 \\
\hline 20 & 6 & 15.491668 & -3.954300 & 1.607276 \\
\hline 21 & 6 & -15.491670 & 3.954296 & 1.607278 \\
\hline 22 & 6 & -5.558984 & 3.713028 & 2.099272 \\
\hline 23 & 6 & 4.340342 & 3.981082 & -1.530326 \\
\hline 24 & 6 & 14.259975 & 3.691242 & -2.147499 \\
\hline 25 & 6 & -19.128509 & -0.684813 & -0.005625 \\
\hline 26 & 6 & -9.157473 & -0.437983 & -0.543012 \\
\hline 27 & 6 & 0.749806 & -0.697900 & -0.001339 \\
\hline 28 & 6 & 10.658761 & -0.437683 & 0.543146 \\
\hline 29 & 6 & -10.658761 & 0.437683 & 0.543146 \\
\hline 30 & 6 & -0.749806 & 0.697904 & -0.001340 \\
\hline 31 & 6 & 9.157472 & 0.437984 & -0.543011 \\
\hline 32 & 6 & 19.128509 & 0.684808 & -0.005626 \\
\hline
\end{tabular}


0.710724

0.535793

0.712811

0.533480

$-0.533483$

$-0.712807$

$-0.535790$

$-0.710728$

$-1.652213$

$-1.486985$

$-1.665653$

$-1.476897$

1. 476894

1. 665656

1.486988

1. 652210

$-0.710727$

$-0.535789$

$-0.712808$

$-0.533483$

0.533481

0.712811

0.535792

0.710723

1. 652210

1.486989

1. 665655

1. 476894

$-1.476897$

$-1.665652$

$-1.486986$

$-1.652214$

1. 334316

0.926521

1. 385554

0.923720

$-0.923720$

$-1.385551$

$-0.926520$

$-1.334320$

$-2.937932$

$-2.506766$

$-2.964277$

$-2.492610$

2. 492606

2. 964281

2. 506770

2. 937929

$-1.334319$

$-0.926519$

$-1.385551$

$-0.923720$

0.923719

1. 385554

0.926521

1. 334315

2.937929

2. 506771

2. 964280

2. 492607

$-2.492610$

$-2.964276$

$-2.506767$

$-2.937933$

2. 255350

3. 237370

2. 264034

3.178529

$-3.178533$

$-2.264031$

$-3.237366$

$-2.255351$

3. 178529

2. 264035

3. 237369

2. 255351

$-2.255352$

$-3.237366$

$-2.264033$
0.131567

0.482206

$-0.104378$

$-0.485818$

$-0.485817$

$-0.104382$

0.482210

0.131565

$-0.595231$

$-0.930852$

0.551393

0.951749

0.951750

0.551388

$-0.930847$

$-0.595233$

$-0.131560$

$-0.482213$

0.104377

0.485820

0.485821

0.104373

$-0.482210$

$-0.131562$

0.595238

0.930843

$-0.551393$

$-0.951746$

$-0.951745$

$-0.551398$

0.930847

0.595236

0.094328

1.031897

$-0.056267$

$-1.033681$

$-1.033681$

$-0.056269$

1.031900

0.094326

$-0.879306$

$-1.774410$

0.802293

1.799339

1. 799341

0.802288

$-1.774405$

$-0.879307$

$-0.094321$

$-1.031902$

0.056264

1.033683

1. 033684

0.056262

$-1.031899$

$-0.094322$

0.879314

1. 774400

$-0.802294$

$-1.799336$

$-1.799335$

$-0.802299$

1. 774405

0.879311

2. 333843

0.243787

$-2.326721$

$-0.376188$

$-0.376187$

$-2.326725$

0.243790

2. 333842

0.376195

2. 326718

$-0.243793$

$-2.333839$

$-2.333839$

$-0.243797$

2. 326722 


$\begin{array}{rrrrr}112 & 34 & 17.891242 & -3.178534 & 0.376193 \\ 113 & 1 & -13.749785 & 4.413520 & 2.776837 \\ 114 & 1 & -3.835282 & 4.928252 & 1.690488 \\ 115 & 1 & 6.062462 & 4.438533 & -2.730223 \\ 116 & 1 & 16.004298 & 4.893579 & -1.788858 \\ 117 & 1 & -16.004297 & 4.893579 & 1.788866 \\ 118 & 1 & -6.062461 & 4.438535 & 2.730217 \\ 119 & 1 & 3.835282 & 4.928250 & -1.690496 \\ 120 & 1 & 13.749787 & 4.413521 & -2.776830 \\ 121 & 1 & -13.749785 & -4.413524 & -2.776828 \\ 122 & 1 & -3.835280 & -4.928245 & -1.690504 \\ 123 & 1 & 6.062459 & -4.438530 & 2.730225 \\ 124 & 1 & 16.004295 & -4.893583 & 1.788863 \\ 125 & 1 & -16.004296 & -4.893583 & -1.788856 \\ 126 & 1 & -6.062460 & -4.438528 & -2.730232 \\ 127 & 1 & 3.835280 & -4.928247 & 1.690496 \\ 128 & 1 & 13.749784 & -4.413524 & 2.776834 \\ 129 & 1 & 20.052421 & 1.254401 & -0.010715 \\ 130 & 1 & 20.052420 & -1.254407 & 0.010719 \\ 131 & 1 & -20.052421 & 1.254402 & 0.010720 \\ 132 & 1 & -20.052421 & -1.254405 & -0.010714 \\ ---------------------------------------------------\end{array}$

Table S28. The optimized Cartesian coordinates of the TSC-based ribbon fused through four-membered ring with $\mathrm{n}=5$ (type III) in the ground singlet state calculated at the B3LYP/6-31(d) level of theory

\begin{tabular}{|c|c|c|c|c|}
\hline \multirow{2}{*}{$\begin{array}{l}\text { Center } \\
\text { Number }\end{array}$} & \multirow{2}{*}{$\begin{array}{l}\text { Atomic } \\
\text { Number }\end{array}$} & \multicolumn{3}{|c|}{ Coordinates (Angstroms) } \\
\hline & & $\mathrm{X}$ & $\mathrm{Y}$ & Z \\
\hline 1 & 6 & -19.219271 & 4.253878 & 0.374831 \\
\hline 2 & 6 & -9.302408 & 4.253341 & -0.312498 \\
\hline 3 & 6 & 0.609045 & 4.253235 & 0.314638 \\
\hline 4 & 6 & 10.520349 & 4.252948 & -0.317091 \\
\hline 5 & 6 & 20.450709 & 4.262338 & 0.226539 \\
\hline 6 & 6 & -20.450709 & -4.262338 & 0.226539 \\
\hline 7 & 6 & -10.520349 & -4.252948 & -0.317091 \\
\hline 8 & 6 & -0.609045 & -4.253235 & 0.314638 \\
\hline 9 & 6 & 9.302408 & -4.253341 & -0.312498 \\
\hline 10 & 6 & 19.219271 & -4.253878 & 0.374831 \\
\hline 11 & 6 & -24.087579 & 0.622148 & -0.286251 \\
\hline 12 & 6 & -14.116893 & 0.627125 & 0.305675 \\
\hline 13 & 6 & -4.205191 & 0.627345 & -0.305167 \\
\hline 14 & 6 & 5.706476 & 0.627404 & 0.305083 \\
\hline 15 & 6 & 15.618137 & 0.626902 & -0.305903 \\
\hline 16 & 6 & -15.618137 & -0.626902 & -0.305903 \\
\hline 17 & 6 & -5.706476 & -0.627404 & 0.305083 \\
\hline 18 & 6 & 4.205191 & -0.627345 & -0.305167 \\
\hline 19 & 6 & 14.116893 & -0.627125 & 0.305675 \\
\hline 20 & 6 & 24.087579 & -0.622148 & -0.286251 \\
\hline 21 & 6 & -19.219271 & -4.253878 & -0.374831 \\
\hline 22 & 6 & -9.302408 & -4.253341 & 0.312498 \\
\hline 23 & 6 & 0.609045 & -4.253235 & -0.314638 \\
\hline 24 & 6 & 10.520349 & -4.252948 & 0.317091 \\
\hline 25 & 6 & 20.450709 & -4.262338 & -0.226539 \\
\hline 26 & 6 & -20.450709 & 4.262338 & -0.226539 \\
\hline 27 & 6 & -10.520349 & 4.252948 & 0.317091 \\
\hline 28 & 6 & -0.609045 & 4.253235 & -0.314638 \\
\hline 29 & 6 & 9.302408 & 4.253341 & 0.312498 \\
\hline 30 & 6 & 19.219271 & 4.253878 & -0.374831 \\
\hline 31 & 6 & -24.087579 & -0.622148 & 0.286251 \\
\hline 32 & 6 & -14.116893 & -0.627125 & -0.305675 \\
\hline 33 & 6 & -4.205191 & -0.627345 & 0.305167 \\
\hline 34 & 6 & 5.706476 & -0.627404 & -0.305083 \\
\hline 35 & 6 & 15.618137 & -0.626902 & 0.305903 \\
\hline 36 & 6 & -15.618137 & 0.626902 & 0.305903 \\
\hline 37 & 6 & -5.706476 & 0.627404 & -0.305083 \\
\hline 38 & 6 & 4.205191 & 0.627345 & 0.305167 \\
\hline 39 & 6 & 14.116893 & 0.627125 & -0.305675 \\
\hline 40 & 6 & 24.087579 & 0.622148 & 0.286251 \\
\hline 41 & 6 & -21.574173 & 0.699177 & -0.183246 \\
\hline 42 & 6 & -11.656231 & 0.689785 & 0.208934 \\
\hline 43 & 6 & -1.744640 & 0.689897 & -0.208470 \\
\hline
\end{tabular}


0.208227

$-0.213238$

$-0.213238$

0.208227

$-0.208470$

0.208934

$-0.183246$

0.163811

$-0.210555$

0.209603

$-0.208855$

0.233857

0.233857

$-0.208855$

0.209603

$-0.210555$

0.163811

0.183246

$-0.208934$

0.208470

$-0.208227$

0.213238

0.213238

$-0.208227$

0.208470

$-0.208934$

0.183246

$-0.163811$

0.210555

$-0.209603$

0.208855

$-0.233857$

$-0.233857$

0.208855

$-0.209603$

0.210555

$-0.163811$

$-0.482285$

0.540655

$-0.539821$

0.539582

$-0.543580$

$-0.543580$

0.539582

$-0.539821$

0.540655

$-0.482285$

0.453578

$-0.539217$

0.537382

$-0.536098$

0.569522

0.569522

$-0.536098$

0.537382

$-0.539217$

0.453578

0.482285

$-0.540655$

0.539821

$-0.539582$

0.543580

0.543580

$-0.539582$

0.539821

$-0.540655$

0.482285

$-0.453578$

0.539217

$-0.537382$

0.536098

$-0.569522$

$-0.569522$

0.536098

$-0.537382$

0.539217

$-0.453578$

1.154761

$-1.141300$ 


\begin{tabular}{|c|c|c|c|c|}
\hline 123 & 34 & 3.001871 & 3.038782 & 1.141806 \\
\hline 124 & 34 & 12.913213 & 3.038097 & -1.143619 \\
\hline 125 & 34 & 22.850182 & 3.036701 & 1.011653 \\
\hline 126 & 34 & -22.850182 & -3.036701 & 1.011653 \\
\hline 127 & 34 & -12.913213 & -3.038097 & -1.143619 \\
\hline 128 & 34 & -3.001871 & -3.038782 & 1.141806 \\
\hline 129 & 34 & 6.909935 & -3.039041 & -1.141300 \\
\hline 130 & 34 & 16.815456 & -3.033198 & 1.154761 \\
\hline 131 & 34 & -22.850182 & 3.036701 & -1.011653 \\
\hline 132 & 34 & -12.913213 & 3.038097 & 1.143619 \\
\hline 133 & 34 & -3.001871 & 3.038782 & -1.141806 \\
\hline 134 & 34 & 6.909935 & 3.039041 & 1.141300 \\
\hline 135 & 34 & 16.815456 & 3.033198 & -1.154761 \\
\hline 136 & 34 & -16.815456 & -3.033198 & -1.154761 \\
\hline 137 & 34 & -6.909935 & -3.039041 & 1.141300 \\
\hline 138 & 34 & 3.001871 & -3.038782 & -1.141806 \\
\hline 139 & 34 & 12.913213 & -3.038097 & 1.143619 \\
\hline 140 & 34 & 22.850182 & -3.036701 & -1.011653 \\
\hline 141 & 1 & -18.709181 & 5.175162 & 0.637650 \\
\hline 142 & 1 & -8.797788 & 5.178461 & -0.572136 \\
\hline 143 & 1 & 1.113669 & 5.178229 & 0.574722 \\
\hline 144 & 1 & 11.024874 & 5.177815 & -0.577845 \\
\hline 145 & 1 & 20.963216 & 5.189708 & 0.461755 \\
\hline 146 & 1 & -20.963216 & 5.189708 & -0.461755 \\
\hline 147 & 1 & -11.024874 & 5.177815 & 0.577845 \\
\hline 148 & 1 & -1.113669 & 5.178229 & -0.574722 \\
\hline 149 & 1 & 8.797788 & 5.178461 & 0.572136 \\
\hline 150 & 1 & 18.709181 & 5.175162 & -0.637650 \\
\hline 151 & 1 & -18.709181 & -5.175162 & -0.637650 \\
\hline 152 & 1 & -8.797788 & -5.178461 & 0.572136 \\
\hline 153 & 1 & 1.113669 & -5.178229 & -0.574722 \\
\hline 154 & 1 & 11.024874 & -5.177815 & 0.577845 \\
\hline 155 & 1 & 20.963216 & -5.189708 & -0.461755 \\
\hline 156 & 1 & -20.963216 & -5.189708 & 0.461755 \\
\hline 157 & 1 & -11.024874 & -5.177815 & -0.577845 \\
\hline 158 & 1 & -1.113669 & -5.178229 & 0.574722 \\
\hline 159 & 1 & 8.797788 & -5.178461 & -0.572136 \\
\hline 160 & 1 & 18.709181 & -5.175162 & 0.637650 \\
\hline 161 & 1 & -25.011476 & 1.139803 & -0.523979 \\
\hline 162 & 1 & -25.011476 & -1.139803 & 0.523979 \\
\hline 163 & 1 & 25.011476 & 1.139803 & 0.523979 \\
\hline 164 & 1 & 25.011476 & -1.139803 & -0.523979 \\
\hline
\end{tabular}

